Alternativas

Cuadernos

de trabajo social 
Dirección

Mª Asunción Martínez-Román (masun.martinez@ua.es)

\section{Secretaria}

Yolanda Domenech López (yolanda.domenech@ua.es)

\section{Comité Editorial/Editorial Board*}

Andrés Arias Astray. Universidad Complutense de Madrid

Antonio Gorri Goñi. Universidad Pública de Navarra

Antonio López Peláez. Universidad Nacional de Educación a Distancia

Carmen Alemán Bracho. Universidad Nacional de Educación a Distancia

Carmen Barranco Expósito. Universidad de La Laguna

Cristóbal Torres Albero. Universidad Autónoma de Madrid

Fernando Casas Mínguez. Universidad de Castilla-La Mancha (Cuenca)

Jorge Garcés Ferrer. Universitat de València

María Asunción Martínez Román. Universidad de Alicante

Natividad de la Red Vega. Universidad de Valladolid

Octavio Vázquez Aguado. Universidad de Huelva

Víctor M. Giménez Bertomeu. Universidad de Alicante

Yolanda Domenech López. Universidad de Alicante

\section{Consejo Científico Asesor/Advisory Board}

Carmina Puig Cruells. Universitat Rovira i Virgili. Tarragona

Clarisa Ramos Feijóo. Universidad de Alicante

Enrique Pastor Seller. Universidad de Murcia

Esther Villegas Castrillo. Universidad de Alicante

Fernando de Lucas y Murillo de la Cueva. Universidad Complutense

José Luis Sarasola-Sánchez Serrano. Universidad Pablo de Olavide. Sevilla

José Manuel Barbero García. Universitat de Girona

Josefa Lorenzo García. Universidad de Alicante

Josefina Fernández Barrera. Universidad de Barcelona

Luís Enrique Alonso Benito. Universidad Autónoma de Madrid

María Carmen Pérez Belda. Universidad de Alicante

María de las Olas Palma García. Universidad de Málaga

María Jesús Uriz Pemán. Universidad Pública de Navarra

María José Escartín Caparrós. Universidad de Alicante

María Luisa Setién Santamaría. Universidad de Deusto

Marta Llobet Estany. Universidad de Barcelona

Santa Lázaro Fernández. Universidad Pontificia de Comillas

Teresa Facal Fondo. Universidad de Santiago de Compostela

\section{Consejo Científico Asesor Internacional/International Advisory Board}

Agnes Koon Chui Law. Center for Social Work Eduation and Research. Sun Yat-sen University. Guangzhou, China. Alberto Acosta Espinosa. Facultad Latinoamericana de Ciencias Sociales, (FLACSO- Ecuador). Quito, Ecuador.

Annamaria Campanini. Università degli Studi di Milano-Bicocca. Milán, Italia.

Darja Zavirsek. University of Ljubljana. Ljubljana, Slovenia.

Göran Therborn. Göteborg University. Göteborg, Sweden.

John Gal. Paul Baerwald School of Social Work and Social Welfare. Hebrew University of Jerusalem. Jerusalem, Israel. Johan Galtung. Transcend. Peace University. A Peace and Development Network.

José Paulo Netto. Universidade Federal do Rio de Janeiro. Rio de Janeiro, Brasil.

Mona Fransehn. Göteborg University. Göteborg, Sweden.

Nino Zganec. University of Zagreb. Zagreb, Croatia.

Philip Mendes. Monash University. Melbourne, Australia.

\footnotetext{
* El Comité Editorial tiene las siguientes funciones. Asistir al editor de la revista, especialmente, en el seguimiento de los trabajos: recepción, evaluación, aceptación y decisión final sobre la publicación del trabajo. Asimismo es responsable del estilo, definición de las normas de presentación de trabajos y de establecer la estructura de la revista.
} 


\section{ALTERNATIVAS \\ Cuadernos de Trabajo Social}


La Revista Alternativas. Cuadernos de Trabajo Social se encuentra actualmente indexada en los siguientes directorios y bases de datos.

\section{SISTEMAS DE EVALUACIÓN}

C.I.R.C. CIRC - Clasificación Integrada de Revistas Científicas

MIPla MIAR - Matriz de información para la evaluación de revistas

IN RECS IN-RECS - Índice de impacto de revistas españolas de ciencias sociales

1éSh RESH - Revistas Españolas de Ciencias Sociales y Humanas

BASES DE DATOS

latindex Latindex - Sistema regional de información en línea para revistas científicas de América Latina, el Caribe, España y Portugal

ISOC - Base de Datos Bibliográficos de Ciencias Sociales y Humanidades del Centro Superior de Investigaciones Científicas (CSIC)

- Dialnet Dialnet - Portal de difusión de la producción científica iberoamericana impulsado por la Universidad de La Rioja

Psicodoc PSICODOC - Base de datos internacional que facilita la búsqueda bibliográfica y el acceso al texto completo de las publicaciones científicas sobre Psicología y otras disciplinas afines

ULRICH'S - Base de datos de publicaciones periódicas internacionales

EBSCO EBSCO. Academic

\section{PORTALES DE DIFUSIÓN}

REDIB REDIB - Red Iberoamericana de Innovación y Conocimiento Científico

4. HISPANA ${ }^{4}$ Hispana - Directorio y recolector de recursos digitales del Ministerio de Educación

OAlster OAIster - La base de datos OAIster

$$
\text { OpenDOAR - The Directory of Open A }
$$

OpenDOAR OpenDOAR - The Directory of Open Access Repositories

Recolecta RECOLECTA - Recolector de Ciencia Abierta

S. DULCINEA Dulcinea - Proyecto coordinado por el Ministerio de Educación y Ciencia para identificar y analizar las políticas editoriales de las revistas científicas españolas

\section{DIRECCIÓN POSTAL}

Alternativas. Cuadernos de Trabajo Social. Universidad de Alicante

Departamento de Trabajo Social y Servicios Sociales. Ap. Correos 99 (03080) Alicante

alternativasts@ua.es

http://rua.ua.es/dspace/handle/10045/5269/

Información estadística relativa al No 24. Año 2017

\begin{tabular}{|l|c|}
\hline Número total de trabajos recibidos / Total number of submissions & 24 \\
\hline Número de trabajos aceptados (\%) / Total number of submissions accepted & 8 (33,33\%) \\
\hline \% de trabajos rechazados / \% of submissions rejected & $11(45,83 \%)$ \\
\hline Trabajos en proceso de revisión y evaluación (\%) / Submissions in review process (\%) & $5(20,83 \%)$ \\
\hline Número medio de revisores por artículos / Average number or reviewers per article & 2 \\
\hline Demora media recepción-revisión / Average time reception-review & 13,38 \\
\hline Demora media aceptación-publicación / Average time acceptance-publication & 48,88 \\
\hline Demora media recepción-publicación / Average time reception-publication & 97,63 \\
\hline \% de trabajos que comunican resultados de investigación originales / \% works reporting results of original research & $(4 / 8) 50 \%$ \\
\hline \% de autores externos al Comité Editorial / \% of external authors (Editorial Board) & $(18 / 18)$ \\
\hline \% de autores externos a la organización editora de la revista / \% of external authors (Publishing Organisation) & $100 \%$ \\
\hline \% de autores extranjeros / \% Foreign authors & $(18 / 18)$ \\
\hline $\begin{array}{l}\text { \% de trabajos financiados por organismos públicos o privados de investigación / \% works funded by public/private } \\
\text { reseach organisation }\end{array}$ & $(100 \%$ \\
\hline
\end{tabular}




\section{ALTERNATIVAS \\ Cuadernos de Trabajo Social}

N. ${ }^{\circ}$ 24. Año 2017

DEPARTAMENTO DE TRABAJO SOCIAL Y SERVICIOS SOCIALES

UNIVERSIDAD DE ALICANTE

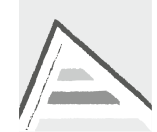

Universitat d'Alacant

Universidad de Alicante 
La publicación de este número ha sido posible gracias a la obtención de una ayuda del Vicerrectorado de Investigación, Desarrollo e Innovación de la Universidad de Alicante.

\section{Universitat d'Alacant \\ Aniversidad de Alicante}

\section{TITULO: ALTERNATIVAS. CUADERNOS DE TRABAJO SOCIAL ISSN: 1133-0473 \\ ISSN electrónico: 1989-9971}

EDITOR: Departamento de Trabajo Social y Servicios Sociales. Universidad de Alicante PERIODICIDAD: Anual

FECHA DE INICIO: 1992

WEB: http://dtsss.ua.es/es/alternativascuadernostrabajosocial/

CORREO ELECTRÓNICO: alternativasts@ua.es

http://publicaciones.ua.es/publica/revistas.aspx?Cod=11

RUA: http://rua.ua.es/dspace/handle/10045/5269

\section{TITLE: ALTERNATIVAS. CUADERNOS DE TRABAJO SOCIAL}

ISSN: 1133-0473

Electronic ISSN: 1989-9971

PUBLISHER: Department of Social Work and Social Services, University of Alicante, Spain PERIODICITY: Annual

START DATE: 1992

WEB: http://dtsss.ua.es/es/alternativascuadernostrabajosocial/

EMAIL: alternativasts@ua.es

http://publicaciones.ua.es/publica/revistas.aspx?Cod=11

RUA: http://rua.ua.es/dspace/handle/10045/5269

Publicaciones de la Universidad de Alicante

Campus de San Vicente s/n

03690 San Vicente del Raspeig

Publicaciones@ua.es

http://publicaciones.ua.es

Teléfono: 965903480

(C) de la presente edición: Universidad de Alicante

ISSN: 1133-0473

ISSN electrónico: 1989-9971

Depósito legal: A 974-2015

DOI: 10.14198/ALTERN

Diseño de portada: candela ink Composición: Marten Kwinkelenberg

\section{@థब}

Los contenidos están bajo una licencia Creative Commons Reconocimiento-NoComercial 4.0 Internacional. Los contenidos pueden copiarse, distribuirse o comunicarse públicamente, bajo las siguientes condiciones generales: Reconocimiento. Debe reconocerse los créditos de la obra de la manera especificada por el autor o el licenciador (pero no de una manera que sugiera que tiene su apoyo o apoyan el uso que hace de su obra). Los términos de la licencia disponibles on-line en: https://creativecommons.org/licenses/by-nc/4.0/ 


\section{ÍNDICE}

1. La maternidad en parejas de mujeres: reformulando el parentesco heteronormativo y biologicista

Motherhood in same-sex female couples: reformulating heteronormative and biological kinship

GLORIA ÁlVAREZ BERNARDO

2. Análisis del modelo de vulnerabilidad para la toma de decisiones en el ámbito de los cuidados de las personas mayores

Analysis of the vulnerability model for decision making related to elderly care 29

Usue Beloki Marañón, Amaia Mosteiro Pascual

3. El cambio en los modelos del trabajo social en salud mental: del modelo rehabilitador al modelo social

Changes on social work models in mental health: from the rehabilitation model to the social model.

Josep CAZOrla PALOMO, BelÉn ParRa RAMajo

4. Propriedades Psicométricas da Geriatric Social Work Competencies

Scale II

Psychometric Properties of Geriatric Social Work Competencies Scale II

FERnANDa DANIEL, Jorge FERREIRA, Rosa mONTEIRO

5. El diagnóstico relacional colaborativo (I)

The collaborative relational diagnosis (I).

Josefa Cardona Cardona, María Elena Cuartero Castañer, JOSÉ FRANCISCO CAMPOS VIDAL

6. Percepciones para la intervención social desde la competencia intercultural de profesionales

Perceptions for social intervention from professionals' intercultural competence. 91

Manuela Ángela Fernández Borrero, FERnANdo Relinque Medina, Susana Martí García 
7. Afrontar el desgaste: cuidado y mecanismos paliativos de la fatiga por compasión

Facing the wear: care and palliative mechanisms of compassion fatigue...... 119 José Francisco CAMPOS VIDAL, JOSEFA CARDONA CARDONA, MARÍA ELENA CuARTERo CASTAÑER

8. Factores sociofamiliares y estancia hospitalaria: la complejidad de la intervención social en el área de la psiquiatría de agudos. Aplicación de la Escala ECISACH-BCN PSMAR

Family and social factors and hospital stay: the complexity of social intervention in the area of acute psychiatry. Application of the ECISACH-BCN PSMAR Scale

LAURA MORRO FERNÁNDEZ

Enfoque y alcance. Directrices para autores/as 161

Focus and scope. Author guidelines 171

Proceso de evaluación por pares 181

Peer Review Process 187 
Cita bibliográfica: Álvarez-Bernardo, G. (2017). La maternidad en parejas de mujeres: reformulando el parentesco heteronormativo y biologicista [Motherhood in same-sex female couples: reformulating heteronormative and biological kinship]. Alternativas. Cuadernos de Trabajo Social, 24, 9-28. https://doi.org/10.14198/ALTERN2017.24.01

\title{
LA MATERNIDAD EN PAREJAS DE MUJERES: REFORMULANDO EL PARENTESCO HETERONORMATIVO Y BIOLOGICISTA
}

\section{MOTHERHOOD IN SAME-SEX FEMALE COUPLES: REFORMULATING HETERONORMATIVE AND BIOLOGICAL KINSHIP}

\author{
GLORIa ÁlVAREZ-Bernardo \\ Instituto Universitario de Investigación de Estudios de las Mujeres y de Género \\ Universidad de Granada \\ gloab@ugr.es
}

\begin{abstract}
Resumen
La maternidad en las parejas de mujeres desafía dos de los principios sobre los que se articula la construcción del parentesco occidental: la heteronormatividad y el énfasis de los vínculos biológicos. El objetivo de este trabajo es conocer cómo afrontan la maternidad estas parejas dentro de unos esquemas tan restrictivos y excluyentes. Se desarrolló una investigación cualitativa basada en la realización de entrevistas semi-estructuradas a 28 mujeres que habían sido madres dentro de una relación de pareja del mismo sexo. Se concluye que las parejas que desean acceder a la maternidad deben sortear una serie de barreras asociadas a la construcción heteronormativa y biologicista del parentesco.
\end{abstract}

Palabras clave: Maternidad; parejas de mujeres; parentesco; heteronormatividad; biología

\section{Extended abstract}

\section{Introduction}

Kinship has been defined as a mechanism responsible for regulating the functioning and organization of western societies (e.g. González Echevarría et al., 2010; Stone, 2008). The nuclear family has been presented as the ideal family model and special emphasis has been placed on descendants being the result of the heterosexual bond of parents (Beck-Gernsheim, 2002; Rivas, 2008). Thus, heterosexuality has been considered as the only legitimate sexuality to the extent that it pursues reproductive ends (Cutas \& Chan, 2012; Lehr, 1999; Pichardo, 2008).

Numerous authors (Rich, 1980; Rubin, 1986) have refuted these ideas that assume that heterosexuality is the organizing and regulating principle of human societies.

\begin{abstract}
Motherhood in female same-sex couples challenges two important aspects in the construction of western parenthood: on the one hand, heteronormativity; and on the other, the emphasis on biological ties. The aim of this work is to analise how these families confront their motherhood attending to such restrictive and exclusive situations. Qualitative research, based on semi-structured interviews, was conducted with 28 women who had planned their motherhood in a same-sex relationship. It may be concluded that female same-sex couples have to face some obstacles that are related to the heteronormative and biological construction of parenthood.
\end{abstract}

Keywords: Motherhood; female same-sex couples; kinship; heteronormativity; biology

In this regard, Butler (2006) refers to heteronormativity as a normative order that begins and is intensified through kinship relationships, specifically through the traditional heterosexual family. Nevertheless, the plurality of family models that have emerged in recent years has meant that the centrality of the traditional family has been rebutted.

One of the aspects that has had a greater impact on this change has been the proliferation of assisted reproduction treatments. Specifically, the role and meaning of what is biological has been one of the principal ruptures that this reproductive technology has caused (Bestard, Orobitg, Ribot \& Salazar, 2003). The range of possibilities that assisted reproduction techniques offer means that kinship is subjected to a continual process of reformulation and negotiation. In other words, the type of 
kinship bond and its intensity are defined in a particular form, taking into account the characteristics of the actual process of family formation (Thompson, 2001). The boundaries between what is cultural and what is bological are becoming vague, and depending on the peculiarities of the process, more or less emphasis is placed on one or another element (Carsten, 2004).

In this idea of kinship construction, daily practice acquires great significance since through this practice, the bonds of kinship are made and strengthened. Following the proposal made by Weeks, Heaphy and Donovan (2001), family kinship is an action that is based, amongst other things, on the "mutual care, the division of labour in the home, looking after dependents" (p. 37). Along with these activities that take place in the home, concomitantly there are those that go beyond domestic boundaries and take place in the public sphere. These stand out because they are a question of ways of being and making family in the eyes of society. Ultimately, they legitimise a group of people as members of the same family unit.

In this continuous being-and-making family, those models that are far from normative patterns become the object of discrimination. In the specific case of female couples, legal requirements prevent or hinder access to assisted reproductive treatments (Bergmann, 2012; Inhorn \& Birembaum-Carmeli, 2008). A similar situation occurs with the adoption procedure, given that some countries exclude same-sex couples, and reserve this right to heterosexual ones (e.g. Goldberg et al. 2008; Hicks, 2000). In Spain, few studies have analysed this discriminatory and exclusionist approach. No study has been found that tackles this issue of adoption. Regarding assisted reproduction, it is worth highlighting the document produced in 2002 by a team of specialists in this field: Criteria for the use of National Health System resources in applying assisted reproductive techniques. What stands out from their conclusions is that, for these professionals, assisted reproduction is a priority right of heterosexual couples, by associating these treatments with the existence of a situation of sterility/infertility. Ultimately, those couples with a non-reproductive sexuality are excluded from these types of treatments. This situation goes hand-in-hand with legislative reforms that are more and more restrictive to same-sex couples (e.g. Order SSI/2065/2014).

\section{Methodology}

The aim of this work is to learn how female couples deal with maternity within a socio-cultural construction of kinship dominated by normative heterosexuality and the privileging of the biological connection with descendants.

Research was carried out between May 2013 and November 2014, using a qualitative focus, and with a total sample of thirteen same-sex female married couples, as well as two divorced women who, as a couple, had planned to become mothers. Two couples attained maternity through adoption, while the rest did so through assisted reproductive treatment.

The data collection technique used was that of semistructured interviews. These were carried out jointly with both members of each couple. Once the interviews were transcribed, the text was analysed using a phenomenological focus, as proposed by Hycner (1985) and Finlay (2014).

\section{Results}

The heteronormative construction of kinship, combined with the lack of preceding examples or models, means that some of the couples interviewed doubted their own ability to be mothers. In this regard, getting into contact with other female couples who were also mothers was decisive for going ahead with their motherhood project. Another of the doubts that these families have revolves around how they are actually going to accomplish the process. The lack of information, the doubts and fears they have, are some of the issues that these couples must face. Using the internet or having contact with other couples who have gone through the same process are some of the strategies that these families develop to overcome their fears and uncertainties.

Once they have dealt with that first stage, these couples must deal with the prejudice and possible rejection of the professionals that work both in assisted reproduction and in adoption. Regarding the latter, some couples who had begun the process of applying for adoption soon after the law legalising marriage between persons of the same sex was passed were rejected as adoptive families. This occurred even though this same law permitted same-sex couples to adopt jointly. The temporal proximity and novelty of the law coming into force meant that many professionals were not aware of it. This was one of the reasons given by the families for the rejection of their suitability as adoptive parents. Meanwhile, those couples who opted for assisted reproductive treatments saw how, depending on the autonomous community where they resided, there were more or fewer legal impediments to being able to do so in the public healthcare system. Faced with this situation, some couples were forced to fund their treatment themselves through private clinics.

Alongside this heteronormative dimension that emerges from speeches and legal texts, some of the female couples had to deal with the biologistic construction of kinship. In this regard, in the assisted reproduction processes the role of the non-biological or non-gestational mother tended to be questioned both by healthcare professionals and by the social environment. This situation caused discomfort for the couple, and the couple members tended to look for strategies to overcome it. The non-biological mother would tend to make up for the lack of a biological bond through certain practices that sought exclusive or almost exclusive contact with her children.

\section{Conclusions}

This study shows that in western societies a biologistic and heteronormative image of kinship still persists, which is perpetuated through social ideology and legal texts. However, families formed by same-sex couples tend to question this construction of kinship, although this transforming potential sometimes puts them in a vulnerable situation. These results are in agreement with those obtained in similar studies (e.g. Bergmann, 2012; Donoso, 2013; Herrera, 2009; Tasker, 2013; Záchia et al., 2011). Considering this panorama, it is necessary for professionals, particularly those in the sphere of social work, to receive specific training so that their actions are not pervaded by the biases described. 


\section{Introducción \\ El peso de la heterosexualidad normativa y la biología en las relaciones de parentesco}

La antropología ha definido el parentesco como un mecanismo encargado de regular y controlar la reproducción humana (e.g. González Echevarría et al., 2010; Stone, 2008). En el caso de las sociedades occidentales, estas prácticas reguladoras han conformado un modelo ideal de familia que se plasma en la familia nuclear heterosexual (Donoso, 2013; Stacey, 2005). En este modelo familiar se asume el carácter heterosexual de la sexualidad que se asocia con un fin reproductivo (Cutas y Chan, 2012; Lehr, 1999; Pichardo, 2008). De este modo, la filiación pasa a ser el resultado del vínculo matrimonial heterosexual, implantando ambos progenitores su huella biológica sobre la descendencia (Beck-Gernsheim, 2002; Rivas, 2008).

Esta construcción cultural del parentesco ha sido rebatida tanto desde la teoría feminista como desde la antropología. En este sentido, la idea de sexualidad reproductora se ha relacionado con el carácter normativo que adquiere la heterosexualidad en las sociedades occidentales (Rich, 1980; Rubin, 1986). De este modo, el matrimonio ha sido definido como una herramienta que se encarga de perpetuar la idea de sexualidad reproductora. Autoras como Butler (2006) sostienen que aquellas estructuras familiares que se alejan de la «heterosexualidad diádica normativa» suponen un peligro para este orden socio-político. No obstante, concluye la autora, el modelo de familia heterosexual está siendo cuestionado al emerger otras estructuras familiares. Estas son el resultado de determinados cambios sociales, entre ellos el uso extensivo de las técnicas de reproducción asistida que permiten desdibujar los límites entre lo social y lo biológico.

Las técnicas de reproducción asistida han jugado un papel clave en la conceptualización de las relaciones de parentesco; una de sus contribuciones ha sido reformular el significado de lo biológico en las genealogías humanas. En consecuencia, la irrupción de la tecnología provoca, de forma simultánea, una ruptura y una sustitución en el orden natural establecido (Bestard, Orobitg, Ribot y Salazar, 2003). Un posicionamiento similar al anterior es el que mantiene Stone (2008) para quien las técnicas de reproducción asistida son una oportunidad para debatir sobre los límites de lo biológico. Para Stone ya no se puede hablar sólo sobre la sustancia sanguínea como un hito central del parentesco sino que deben contemplarse otros elementos como la genética. Ciertos procesos reproductivos (por ejemplo, la donación de óvulos) provocan una fragmentación en la reproducción, esto es: la mujer que dona frente a la mujer que gesta. La primera tiene una conexión genética con la descendencia 
mientras que la segunda queda vinculada por los lazos biológicos asociados al embarazo, parto y puerperio.

En este contexto de posibilidades se produce lo que Thompson (2001) denomina una «naturalización estratégica», es decir, una práctica discursiva a través de la cual se «reclaman o reniegan los vínculos genealógicos, de la sangre o los genes» (p.178). Unas conclusiones similares a las de Thompson son las que sostiene Carsten. En su obra After kinship (2004), la antropóloga plantea que la tecnología reproductiva ha provocado que los límites entre lo natural y lo cultural se vuelvan difusos. Su propuesta para esta «etapa posterior al parentesco» pasa por una «coreografía» de elementos (biológicos y sociales) que interfieren de forma orquestada en la definición de los sistemas genealógicos.

Al hilo de lo expuesto, el parentesco debe interpretarse como un proceso de construcción en el que los elementos que intervienen son resignificados según las necesidades. Como parte de esa construcción, las prácticas diarias son las que forjan y dan sentido a las relaciones interfamiliares; dentro de estos esquemas conceptuales se sitúa la propuesta de Weeks, Heaphy y Donovan (2001). Este equipo identifica la familia como un «verbo» que se materializa en las prácticas diarias que siguen sus integrantes: «apoyo mutuo, división de las tareas domésticas y cuidado de las personas dependientes» (p.37). Junto con estas acciones que se circunscriben a la esfera privada, coexisten aquellas otras que tienen que ver con la visibilidad de la estructura familiar en el ámbito público. Por ello, Weeks y su equipo identifican el parentesco con la acción, con «hacer cosas de familia», mostrándose bajo estos parámetros en la esfera pública. Las familias encabezadas por parejas del mismo sexo son expresiones de hacer familia que crean «patrones de vida los cuales dan nuevos significados a sus relaciones familiares» (p.50). Sin embargo, estas familias carecen de modelos en los que identificarse y reconocerse y, por tanto, deben buscar fórmulas en las que «auto-inventarse» (p.43) con el fin de ganar legitimidad entre y para sí.

\section{La ruptura con la norma: las familias formadas por parejas de mujeres}

El predominio de lo heterosexual en los discursos e imágenes hegemónicas se traduce en todo un conjunto de problemáticas que deben afrontar las familias formadas por parejas de mujeres en su camino y tránsito por la maternidad. Uno de esos problemas se relaciona con las limitaciones legales que algunos países establecen en ciertos procesos como la adopción o los tratamientos de reproducción asistida. En materia de adopción, algunas legislaciones estatales restringen este derecho a las parejas heterosexuales y/o a hombres y mujeres solteras, sin posibilidad de que las parejas del mismo sexo puedan acceder a 
la filiación conjunta. Esta limitación suele producirse en aquellos contextos en los que no es posible el matrimonio entre personas del mismo sexo, por lo que algunas parejas optan por la adopción a título individual, ocultando su relación (Goldberg, Downing y Sauck, 2008). En otros países en los que la legislación es favorable, algunas parejas sienten miedo e inseguridad porque consideran que su opción sexual puede ser una fuente de estigma y discriminación (Gianino, 2008). En este sentido, es preciso advertir que la legislación española en materia de adopción permite que las parejas casadas del mismo sexo adopten en igualdad de condiciones que las parejas heterosexuales ${ }^{1}$.

En lo que respecta al proceso de adopción, algunas investigaciones (Hicks, 2000) advierten que en las entrevistas con las y los profesionales se reproducen esquemas heterosexuales como, por ejemplo, las preguntas que ahondan en una distribución genérica de las tareas domésticas. Esa ruptura con los esquemas binarios suscita dudas entre los y las evaluadoras acerca de las capacidades de las parejas del mismo sexo para criar y educar a menores. Como consecuencia, estos pensamientos se traducen en la implementación de una serie de criterios que algunas agencias estadounidenses de adopción ponen en práctica para privilegiar a las parejas heterosexuales (Brown, Samlling, Groza y Ryan, 2009; Goldberg et al., 2008). No se han encontrado investigaciones que analicen la adopción en el ámbito español por lo que se desconoce cuál es el proceso que deben afrontar las parejas del mismo sexo a este respecto.

En relación a los tratamientos de reproducción asistida, las parejas de mujeres se enfrentan a una serie de barreras tanto en el ámbito sanitario como en el legal y, de forma genérica, en el ideario social. Estas barreras han adoptado la forma de recomendaciones, normas y leyes que tratan de restringir $y$, en algunos casos, impedir el acceso a este tipo de tecnología reproductiva (e.g. Bergmann, 2012; Inhorn y Birembaum-Carmeli, 2008). En lo relativo al ámbito médico, distintos estudios han puesto de manifiesto la preferencia que existe hacia las parejas heterosexuales como legítimas beneficiarias de esta tecnología reproductiva (Johnson, 2012; Tasker, 2013). En el caso español destaca el documento: Criterios para la utilización de los recursos del Sistema Nacional de Salud en la aplicación de las técnicas de reproducción humana asistida, firmado en el año 2002 por especialistas de la materia. En sus conclusiones se afirma que «la reproducción forma parte del proyecto de vida de una pareja y ésta es

1. La aprobación de la Ley 13/2005, de 1 de julio, por la que se modifica el Código Civil en materia de derecho a contraer matrimonio contempla la equiparación de derechos entre parejas del mismo y de distinto sexo. Este aspecto se hace equiparable al acto de adopción, en los siguientes términos: «El matrimonio tendrá los mismos requisitos y efectos cuando ambos contrayentes sean del mismo o de diferente sexo». 
la razón que la hace trascendental para aquélla y para la sociedad» (2002:13). A pesar de las virtudes asociadas a la reproducción y a la descendencia, este equipo de profesionales reserva ese derecho en exclusiva a las parejas heterosexuales al afirmar que los tratamientos de reproducción deben orientarse a «los miembros de una pareja afecta de esterilidad/infertilidad» (2002:13). Consecuentemente, aquellas parejas que sin ser estériles o infértiles no pueden acceder a la maternidad biológica quedan excluidas de la tecnología reproductiva, entre otros motivos por su opción sexual.

En ocasiones, ese ideario que pervive entre las y los profesionales de la reproducción asistida encuentra el respaldo legislativo. Hay países como Italia o Austria que limitan los tratamientos reproductivos a las parejas heterosexuales casadas o en una relación análoga al matrimonio (Peramato, 2013). Mientras que otros países, como España, no establecen restricciones por orientación sexual ni estado civil a la hora de acceder a la tecnología reproductiva ${ }^{2}$. No obstante, en el caso español, recientes reformas legislativas han abierto la posibilidad de que los distintos sistemas sanitarios autonómicos reserven este derecho a las parejas heterosexuales ${ }^{3}$. En este sentido, la Orden SSI/2065/2014 ha señalado que la financiación pública de este tipo de tratamientos sólo debe contemplarse cuando haya una necesidad o fin terapéutico asociado a un problema en la capacidad reproductiva que haya sido diagnosticado o cuando no se haya logrado un embarazo tras doce meses de relaciones sexuales. Estos criterios asumen la heterosexualidad como la lógica dominante, excluyendo a aquellas mujeres que, sin tener un problema reproductivo, forman parte de una relación de pareja del mismo sexo.

Esta idea de la heterosexualidad normativa que, como se ha expuesto, prevalece en ciertos discursos médicos y legales también tiene su correspondencia en el ideario social. Por este motivo, son comunes las campañas que tratan de deslegitimar el uso y acceso a la tecnología reproductiva por parte de parejas de mujeres. Uno de los argumentos recurrentes tiene que ver con los peligros

2. La Ley 14/2006, de 26 de mayo, sobre técnicas de reproducción humana asistida reconoce el derecho de toda mujer a someterse a este tipo de tratamientos. En el artículo 6.1 se señala que: «Toda mujer mayor de 18 años y con plena capacidad de obrar podrá ser receptora o usuaria de las técnicas reguladas en esta Ley, siempre que haya prestado su consentimiento escrito a su utilización de manera libre, consciente y expresa. La mujer podrá ser usuaria o receptora de las técnicas reguladas en esta Ley con independencia de su estado civil y orientación sexual.

3. La Cartera de Servicios Comunes del Sistema Nacional de Salud regulada por el R.D. 1030/2006, establecía que podían ser beneficiarias de los tratamientos de reproducción asistida las parejas con problemas de esterilidad o cuando hubiese una indicación clínica establecida. Bajo esta «indicación», las parejas de mujeres podían obtener cobertura sanitaria pública, sin necesidad de que hubiese un diagnóstico de esterilidad. 
para su futura descendencia, en concreto: la ausencia de un padre (Bryld, 2001) o el propio bienestar de las y los menores por carecer de referentes genéricos binarios (Robinson, 2002).

En los tratamientos de reproducción asistida dirigidos a parejas de mujeres no sólo entra en juego la lógica heterosexual normativa sino también el papel y el significado de la biología. En lo que respecta a este último punto, es una de las integrantes de la pareja quien se somete al tratamiento reproductivo y, en consecuencia, vive la parte física de la maternidad. Esa conexión a través del embarazo es reforzada por el ideario colectivo que tiende a privilegiar a la madre gestante respecto a la que no vive esa dimensión de la maternidad. La madre gestante cuenta con una conexión que «le viene dada» (Sullivan, 2001: 233) mientras que la madre no gestante debe construir un vínculo ante una sociedad que se muestra exigente sobre este asunto. La lógica que subyace a este planteamiento es la construcción cultural que privilegia el vínculo biológico sobre cualquier otro (Hayden, 2008). Ante la ausencia de referentes externos, la madre gestante debe construir discursivamente tales vínculos: «explicar, defender u ocultar -en suma fabricar- la relación con su hijo y su identidad social con los otros» (Sullivan, 2001: 234). Como señala Bos (2013), la ausencia de ese lazo biológico es lo que obliga a las madres no gestantes a buscar una «justificación parental» (p.23). Esta situación se agrava aún más en aquellos casos en los que la legislación refuerza ese modelo biologicista, desposeyendo a la madre no gestante de cualquier tipo de reconocimiento o derecho con respecto a su descendencia (Ben-Ari y Livni, 2006).

\section{Metodología}

El objetivo que persigue este trabajo es conocer cómo afrontan la maternidad las parejas de mujeres dentro de una construcción sociocultural del parentesco dominada por la heterosexualidad normativa y el privilegio de la conexión biológica con la descendencia.

La investigación se desarrolló entre mayo de 2013 y noviembre de 2014. Se abordó desde un enfoque cualitativo contando con una muestra intencional formada por 13 parejas de mujeres casadas y dos mujeres divorciadas que, en su momento, plantearon su maternidad como un proyecto de pareja. En lo que respecta a las vías de acceso a la maternidad: dos parejas lo hicieron mediante un proceso de adopción nacional mientras que el resto optó por tratamientos de reproducción asistida. El perfil sociodemográfico fue bastante homogéneo en lo que se refiere a nivel de estudios, poseyendo la mayoría de las informantes una titulación universitaria; y a nivel económico, oscilando la media de ingresos familiares mensuales entre los 1.801 y los 2.400 euros. La selección de la 
muestra fue un proceso complejo debido a su carácter de «población oculta» (Heckathorn, 1997). De este modo, las primeras entrevistas se realizaron a personas próximas de la red personal de la investigadora así como contactos proporcionados por distintas asociaciones y colectivos LGTBI (Lesbianas, Gays, Transexuales, Bisexuales e Intersexuales) para, posteriormente, ir completando la muestra mediante un procedimiento de bola de nieve. Como combinación de los factores anteriores, las personas entrevistadas se agruparon en las siguientes comunidades autónomas: Andalucía, Asturias y Extremadura. El tamaño final de la muestra se determinó por saturación teórica (Ruiz, 2012).

El enfoque cualitativo en el estudio de las relaciones de parentesco ha sido avalado por distintas investigaciones (Ganong y Goleman, 2014). Una de sus virtudes es dar voz a aquellos modelos familiares marginales ya que, mediante las entrevistas en profundidad, sus integrantes pueden expresar sus puntos de vista acerca de las interacciones internas y externas. Como técnica de recolección de datos se empleó la entrevista semi-estructurada. Antes de iniciar la entrevista, se solicitó el consentimiento a las informantes y se garantizó la confidencialidad de los datos, así como su anonimato mediante el empleo de nombres ficticios. Tras recabar su consentimiento, se inició la entrevista cuyo guión se basaba en preguntas generales y otras específicas. Las primeras tenían que ver con aspectos importantes de su biografía (familia de origen, crianza y educación, redes de amistades, entre otras) mientras que el segundo bloque se orientaba a aquellos aspectos concretos de su modelo familiar. Con carácter general, las entrevistas fueron realizadas en el domicilio de las informantes y, excepcionalmente, en espacios públicos. Su duración osciló entre los 90 y los 120 minutos.

Las entrevistas fueron grabadas y posteriormente transcritas en su totalidad. Los textos se analizaron desde una perspectiva fenomenológica, es decir, tratando de averiguar el significado subjetivo que las informantes conferían a aquellas cuestiones sobre las que estaban siendo preguntadas. Para ello se siguió el modelo de análisis propuesto por Hycner (1985) y Finlay (2014). Este modelo consiste en la búsqueda de unidades de significado mediante una reducción de la información obtenida. Como señala Finlay, en esta etapa «los datos son transformados en significados» (p.125). Esa búsqueda de significados se realizó con ayuda del programa informático N-Vivo, versión 10. De este modo, se sistematizaron los criterios para identificar las distintas unidades de significado. Una vez seleccionadas estas unidades, el siguiente paso consistió en su agrupación en conjuntos más amplios e integradores. En la última etapa del proceso de análisis se definieron los temas de investigación. A continuación, se exponen los resultados relativos al tema de investigación: «proceso de 
constitución familiar», en el que se integran agrupaciones de significado que versan sobre los procedimientos de reproducción asistida y adopción.

\title{
Resultados
}

«Nos dieron la espalda: hablaban de parejas heterosexuales». El peso de la heteronormatividad en el acceso a la maternidad en parejas del mismo sexo.

El peso de la heteronormatividad se hace latente cuando estas parejas se cuestionan que, por su composición, no van a poder asumir sus funciones parentales. La falta de referentes unido a la presión social que deslegitima a este tipo de parejas hace que sean muchas las dudas que les asaltan antes, durante y tras el acceso a la maternidad. Algunas de las mujeres entrevistadas comentaban que conocer a otras parejas de mujeres que ya eran madres fue el empujón decisivo para iniciar su proyecto. Este fue el caso de Lorena que, tras hablar con la hermana de una amiga que junto con otra mujer eran madres de una niña y un niño, se decidió a iniciar el tratamiento de reproducción asistida:

\begin{abstract}
Sí, una amiga que vive aquí que, a partir de entonces, yo le fui perdiendo miedo al hecho de tener un hijo con una pareja de dos mujeres, o de dos chicos. Porque ella era de Bilbao, y entonces la hermana venía con, primero con el niño y luego ya con los dos niños, un niño y una niña, y su pareja, de vacaciones. Y yo los veía unos niños tan felices, $\tan$ sanos, $\tan$ lindos. Ella me contaba que no tenía ningún problema pero, aún así, yo pensaba y, de hecho, así es que en el País Vasco van mucho más adelantaos que en Andalucía. Sobre todo en temas así tan pioneros, ¿no? ...Y, luego, un día yo iba tranquilamente para el trabajo y me dio el flash de que iba a tener un hijo. Iba a tener un hijo, sí o sí, por encima de todo ¿no? (Lorena, 59 años)
\end{abstract}

La ruptura del «orden natural» de la reproducción hace que estas familias busquen alternativas mediante otros procesos como son las técnicas de reproducción asistida o la adopción. Sin embargo, la falta de referentes próximos provoca que muchas parejas se sientan desorientadas a la hora de iniciar tales procedimientos. El recurso a Internet se convierte en una alternativa a través de la cual se puede acceder a información detallada así como a experiencias personales que sirven de guía durante el camino hacia la maternidad. Blanca y Marta desconocían los pasos que debían seguir para ser madres, en concreto, los aspectos legales que acompañaban esta decisión. El hallazgo de un blog con información sobre estos asuntos resultó ser de gran ayuda, tal y como explicaba Blanca. Ella, por ejemplo, ignoraba que tenía que casarse con Marta si ambas querían tener un reconocimiento legal sobre su futura descendencia:

Nosotras no sabíamos que nos teníamos que casar... Nosotras, ella ha estado toda la vida en este mundo y no conocía ninguna asociación. Yo ni siquiera 
sabía que existían asociaciones. Yo un día indagando en Internet por el tema de la inseminación para dos chicas y tal, pues, encontré un blog, un blog de dos chicas de Valencia. Yo ya empecé a hablar con ellas, abrí yo un blog, esto y lo otro. Y hicieron una reunión de familias homomaternales. Y, entonces, le digo: «vamos a ir Valencia» « ¿cómo?». «Las chicas estas que he conocido por Internet van a hacer un encuentro y tal, y nos vamos a ir. Que nos recogen en la estación, bien. Que no, pues un fin de semana en Valencia». (...) Y, efectivamente, nos fuimos a Valencia. Allí estaban a esperarnos en la asociación. No nos dejaron ni a sol ni a sombra. Y, claro, ahí fue donde descubrimos que nos teníamos que casar antes de que naciera el bebé. Y nosotras: «¿cómo?» (Blanca, 38 años)

Tras solventar algunas de sus dudas y miedos acerca de sus posibilidades, las parejas entrevistadas comenzaron sus respectivos procedimientos de adopción o tratamientos de reproducción asistida. En lo que respecta a la adopción, las informantes iniciaron el trámite una vez aprobada la Ley 13/2005 que permite el matrimonio entre personas del mismo sexo. Con esta reforma legislativa ambas cónyuges pueden solicitar la adopción conjunta de su futura descendencia. Por ese motivo, una vez que Cristina y Alba se casaron, decidieron iniciar todo el procedimiento de adopción puesto que con anterioridad, como mujeres solteras, no se habían planteado esa posibilidad:

Eso nosotras la tuvimos en cuanto aprobaron la Ley. Yo hice una copia rápida del Boletín que por ahí andará. Y yo puse con un fluorescente todo lo que podíamos, pues, por eso dijimos: «allá voy». (Cristina, 53 años)

Una vez iniciado el trámite administrativo, y como parte del mismo, esta pareja fue entrevistada por diferentes profesionales que debían velar por su grado de idoneidad en tanto que familia adoptiva. Tras las entrevistas, Alba y Cristina recibieron una carta en la que se les denegaba la adopción tras un informe negativo de idoneidad. Ambas informantes relataban el trago amargo que supuso esa notificación, al considerar que estaban siendo invalidadas para el ejercicio de la maternidad, un deseo que perseguían desde hacía mucho tiempo. Como explicaba Alba:

Yo no me lo creía, nos vino a Correos y fuimos a buscar la carta certificada. Yo no me podía imaginar que te dijeran que no eras idónea para ser madre. Yo me quedé: «¿Cómo puede ser?» Pues nos quedamos (...) quizás, la primera vez, vamos, lo tengo clarísimo, fue demasiado pronto. Entonces, esos miedos, lo que te decimos. Y no sólo miedos, sino gente que cada uno piensa. Eso es un grupo de técnicos y cada uno piensa de su forma. (Alba, 51 años).

Para esta pareja, el informe negativo de idoneidad estaba relacionado con la falta de experiencia de las y los profesionales debido a la reciente aprobación del matrimonio entre personas del mismo sexo. Desde el punto de vista de la 
pareja, la escasa familiaridad con estas temáticas, rodeada de los estereotipos y prejuicios en torno a las relaciones del mismo sexo, pudo actuar como barrera en los primeros procesos de adopción. Para Cristina, los argumentos esgrimidos para desestimar el procedimiento eran poco sólidos puesto que giraban en torno a: ocultar su opción sexual a un tío octogenario y su incapacidad para defender a sus hijos o hijas ante posibles problemas:

Yo se me ocurrió decir que el único que vivía era mi tío. Me dijo, me preguntó, la psicóloga que nos entrevistó, que si no habíamos sido capaces de decírselo. Y yo le dije que no. Que yo no había sido capaz de decirle a mi tío que nos habíamos casao. Es así. Son los sentimientos, son lo que son. y tampoco porque yo si tenía que dar la cara por mis hijos tampoco la iba a dar. (Cristina, 53 años)

Una situación similar a la descrita fue la que vivieron Rebeca y Sara que, en el año 2006, iniciaron el trámite de adopción de la niña que tenían en régimen de acogida. La contradicción de la sentencia que desestimaba la adopción, unida a los comentarios de un abogado que había participado en el proceso judicial, puso de manifiesto que se trataba de un caso de homofobia. Tal y como explicaba Rebeca:

Además, entre pasillo, lo voy a decir clarito porque es así, y nos lo transmitió así el letrado de la Junta, se habló de que éramos una pareja homosexual. Y había ciertas, había ciertos prejuicios, ¿vale? Entonces, fíjate hasta qué punto había prejuicios. (Rebeca, 50 años)

Tanto en un caso como en el otro, las parejas decidieron que lo mejor era dejar pasar el tiempo hasta que en el discurso social calase la imagen de las familias encabezadas por parejas del mismo sexo. Consideraban que la «normalización» de este tipo de unidades familiares contribuiría a reducir o eliminar los prejuicios y estereotipos que los equipos de profesionales pudiesen tener a este respecto. De este modo, tras varios años de tregua, ambas parejas reiniciaron sus respectivos procedimientos de adopción con un resultado favorable. Los argumentos que inicialmente se habían dado para deslegitimar a estas parejas desaparecieron en el segundo intento:

Incluso, además, me acuerdo que se lo dijimos ya, después ya, cuando fuimos la segunda ve que fuimos y nos dijeron que ya sí éramos idóneas, y comentamos: «¿Qué madurez hay de allí a entonces?» Porque entonces también teníamos ya nuestros cuarenta y tantos años. ¿Sabes lo único que hay? Cinco años de nuestra vida que hemos perdido y que han perdido los niños de estar con nosotras. Eso es lo único que ha pasao. (Cristina, 53 años)

Junto con la adopción, otro de los caminos para acceder a la maternidad es a través de los tratamientos de reproducción asistida. Entre las 11 parejas que accedieron a la maternidad a través de esta vía, se vivieron algunas situaciones 
en las que se reforzaban los imperativos heteronormativos y biologicistas que definen la construcción occidental del parentesco. Los criterios fijados por ciertos gobiernos autonómicos han excluido a las parejas de mujeres de su cartera de servicios en materia de reproducción asistida. Por tanto, se está produciendo una gran disparidad entre autonomías lo que acaba generando discriminación en función del lugar de residencia. Rosa y Raquel, residentes en Asturias, acudieron a la consulta médica para iniciar el tratamiento cuando se encontraron con la negativa del especialista al intuir éste que se trataba de una pareja de mujeres. La Consejería de Salud había decidido atender exclusivamente a aquellas parejas heterosexuales diagnosticadas estériles. No se contemplaban otros supuestos como la «indicación clínica establecida» regulada en la Carta de Servicios Comunes del Sistema Nacional de Salud que permitía a las parejas de mujeres beneficiarse de los tratamientos de reproducción asistida. Estas eran las explicaciones que Raquel daba acerca de su periplo por el sistema sanitario público:

Que él [el médico] llevaba años atendiendo a mujeres lesbianas en la unidad y que no había, que él no tenía ningún problema, que era algo que venía de la Consejería, que había tenido varias reuniones y que ellos pedían que la mujer fuera...fuera estéril. Y yo decía: «Pero...pero vamos a ver, si habéis estado atendiendo. Vamos a ver, si es que ella no me puede dejar embarazada». (...) Pero ellos sólo hablaban de la esterilidad, la indicación establecida se la dejaban, o sea, la omitían por completo y, y lo que argumentaban es que yo no era estéril (...) Porque, vamos a ver, una pareja heterosexual cuando, por ejemplo, el varón tiene, imaginemos, pocos espermatozoides, no es que sea imposible que la deje embarazada, sino que hay una menor probabilidad pero sí les atienden. En cambio yo que estoy con una mujer que es imposible que me dejen embarazada...no lo consideran. (Raquel, 33 años)

Estos antecedentes hicieron que ciertas parejas descartasen ser atendidas en el sistema público ante su temor a ser expulsadas del mismo, por lo que optaron por acudir a una clínica privada y costearse el tratamiento. Esta decisión tampoco resultó sencilla ya que algunas tuvieron que dedicar parte de sus ahorros a satisfacer ese deseo. No obstante, ese cambio en la ruta asistencial suponía un gran esfuerzo para algunas de las informantes ya que disponían de unos ingresos económicos limitados. Ese fue el caso de Victoria y Rocío que sabían que en su comunidad autónoma había parejas a las que se les había denegado el tratamiento en la Seguridad Social y, tras mucho esfuerzo, sumaron la cantidad necesaria para comenzar con el tratamiento privado:

Porque la Seguridad Social justo, creo, no sé si fue ese año, eh (...) hubo casos de la asociación [LGTB] que denunciaron, eh, nos enteramos, yo creo que fue ese año. Yo contaba con que no lo cubría la Seguridad Social, no lo habíamos 
pensado y después que si que la Seguridad Social tardaba mucho y no sé qué. (Victoria, 35 años)

Teníamos ahorrado porque ya teníamos pensado empezar porque nosotras no es que dijeras: «ahorramos dos meses». No, estuvimos ahorrando un buen tiempo. (Rocío, 38 años)

Estas situaciones contrastan con los relatos de otras parejas residentes en comunidades autónomas en las que las políticas públicas sanitarias eran más favorables a las parejas del mismo sexo. Quienes tuvieron cobertura pública lo consiguieron en aquellas autonomías en las que se garantizaba este derecho, produciéndose una gran arbitrariedad entre parejas en función del lugar de residencia. De ello dan prueba los testimonios de Tania y su pareja:

Estábamos en un momento en que en Extremadura no había duda pero empezó a haber casos que tenían problemas y tal (...) contactamos con las distintas, con los distintos organismos que estaban implicaos dentro de la inseminación artificial dentro del sistema público de salud que es MUFACE con las clínicas, o sea, con las personas que son funcionarias. Y, luego, el Instituto de Reproducción Asistida de Extremadura y...en realidad, conocí cuál era el protocolo, que no había problemas, que había problemas, sobre todo, a la hora de derivación en Atención Primaria, pero sabiendo cuál es el recorrido legal y sabiéndole explicar a la gente las cosas, cuál es el itinerario, no había problema. La verdad que fue, fue bastante ágil desde el primer momento. Nos derivaron al ginecólogo, llegamos a la consulta, hicimos las pruebas médicas y fue rápido, De hecho, íbamos con la intención de si teníamos algún tipo de problema, o sea, evidentemente denunciarlo. (Tania, 31 años)

\section{«¿Por qué tienen tanto interés en saber quién ha parido?»}

\section{Cuestionamientos sobre la dimensión biológica de la maternidad en parejas de mujeres}

La mediación de lo biológico en los procesos de reproducción asistida provoca una disparidad entre quienes afrontan la parte física de la maternidad (madre gestante) y quienes no (madre no gestante). De los comentarios de las informantes se deduce que existe un interés y presión social por conocer quién de las dos es la que ha gestado. Este hecho lo relacionan con el privilegio que se confiere a aquella madre que tiene una conexión biológica con su descendencia, en detrimento de la que no lo tiene. En este sentido, las preguntas encaminadas a averiguar quién de las dos era la madre gestante solían generar gran malestar en la pareja:

Eso me molesta mucho. De hecho, me he pillado como empieza a decirlo: «coño, ¿por qué te lo tengo que decir si somos iguales de madre?» «ipor qué tengo que decir quién?» Porque te siguen diciendo: «¿quién ha parido?» Ya ahora como me paro y digo: «no te importa, ¿no?» Pero la verdad que la gente 
lo pregunta mucho. Pero he notado también que, incluso, en este entorno [familias del mismo sexo] la gente pregunta, pero más bien por un interés en el proceso (...) Así que, en este sentido, del entorno no me enfado. Pero sí nos preguntan mucho. Cuando estábamos por ahí un fin de semana fuera ¿dónde fue? En Cáceres, en la montaña, en un pueblo chiquinino, en un bar e insistió el hombre: «pero ¿quién ha parido?» (Miriam, 37 años)

Que a mí, por ejemplo, es una de las preguntas que menos me gusta de la gente, que yo sé que lo hacen sin mala...pero es que es un concepto que tenemos: «ah, es nuestro hijo y tal» ¿Quién de las dos lo ha parido?» «Y qué más da. Qué más da. Si es que eso da igual.» (Teresa, 44 años)

Ante esta tesitura, las informantes comentaban que trataban de buscar estrategias por medio de las cuales equilibrar las diferencias que se producían en el plano biológico. De este modo, algunas de las madres no gestantes explicaban cómo habían encontrado tareas, espacios o tiempos para reforzar su relación con sus hijas e hijos y, simultáneamente, diferenciarse de lo que podían aportar las madres gestantes, por ejemplo: el embarazo y el parto o la lactancia. En este sentido, Nerea generó un vínculo físico muy fuerte con su hijo a través del contacto directo, una situación equiparable a la que se produce durante la lactancia materna. Esta estrategia fue clave a la hora de intensificar la relación materno-filial que, desde su punto de vista, era más fuerte que la que existía con la madre gestante:

La diferencia puede estribar en los apegos del bebé. Quiero decir que, claro, si la madre biológica le da el pecho va a hacer un apego que se puede sustituir de otra manera. Que se puede sustituir que yo, mi ex [pareja] no le dio el pecho, pero yo lo que hacía era desnudarme de aquí para arriba de bebé y le desnudaba y le ponía en el pecho, cerca del corazón para que, cantarle a la tripa, hablar mucho con él, tal. Y el apego es que lo hizo conmigo. (Nerea, 60 años)

Precisamente, la lactancia fue un factor determinante para algunas informantes que veían en ese acto una de las principales diferencias entre una y otra madre. Por ello, algunas madres no gestantes optaron por una lactancia inducida con el objeto de minimizar las posibles diferencias trazadas por el hecho biológico. Verónica y Miriam fue una de las parejas entrevistadas que optó por ese procedimiento:

Lo que más podía diferenciarnos, en lo que más podía yo que ella no, es en dar el pecho y como eso lo tenemos también compartido. (Verónica, 32 años)

Pero está guay. Empezamos cuando ella [su hija] tenía ya un mes, cuando tenía un mes porque no lo teníamos muy claro antes. Yo tampoco lo tenía muy claro pero si queremos compartirlo (...) Antes pensaba: «eso es mucha responsabilidad física». Que no sabía si quería esa dependencia física, pero me encanta. Es raro que me guste tanto, pero me gusta mucho. Es muy bonito. (Miriam, 37 años) 
Por otra parte, la personalidad y el carácter particular de cada una de las madres provocó una especialización en el tipo de tareas lo que, a su vez, determinó un cuidado y una crianza diferenciada. En este sentido, opinaban que sus hijas e hijos eran capaces de discernir e identificar a sus madres en función de sus necesidades, de lo que necesitaban en un momento concreto. Raquel y Rosa tenían una relación diferente con su hijo ya que la primera se encargaba de los cuidados físicos del menor mientras que la segunda cubría aquellos aspectos de carácter lúdico:

Raquel es muy rígida para muchas cosas. Es muy, muy madre metódica, perfeccionista, organizada y ordenada. Y, yo creo que lo va a ser así para todo. Yo soy de quitarle importancia a cosas, a todo, de hacer una risa, a veces, incluso, cuando no debo. La, la pongo de los nervios, a veces. Pero, bueno, está ahí el contrapunto. Ella le da unas coas y yo le doy otras. Además es que él las identifica. Ella me dice muchas veces: «jo, mira el niño no se ríe conmigo como contigo». «Ya, ni a mí me mira con esos ojos de $m a, m a, m a \ldots »$ (Rosa, 41 años)

Un caso similar al descrito por las informantes anteriores era el de Patricia y Tania quienes aseguraban mantener una relación diferenciada con su hija. De nuevo, los rasgos específicos de la personalidad de ambas madres actuaban como factores decisivos en sus interacciones:

A la hora de dormir la busca a ella [Patricia] y a la hora de despertarse me busca a mí. Y es que esos roles estarán. Es que, como personas distintas que somos, sabrá encontrarlos en un lado y otro. Habrá alguien que sea más permisivo o menos permisivo, o más... (Tania, 31 años)

\section{Discusión y Conclusiones}

Al inicio de este artículo se planteaba la necesidad de conocer cómo afrontan la maternidad las parejas de mujeres. Al hilo de los relatos de las informantes, se ha constatado que aún perduran estructuras socio-políticas que tienden a reforzar e idealizar el modelo de familia heterosexual, excluyendo aquellas otras formaciones familiares que se escapan de ese esquema. Estos modelos familiares ponen en entredicho la propia organización y funcionamiento de la sociedad y, en particular, la hegemonía de la heterosexualidad (e.g. Grau, 2010; Yanagisako y Collier, 1999) y su vinculación con una sexualidad reproductora (Cadoret, 2013; Rivas, 2008; Rubin, 1986). Los cambios legislativos introducidos en materia de adopción y las posibilidades ofertadas por las técnicas de reproducción asistida han permitido rebatir ese binomio que vincula heterosexualidad y reproducción (Stone, 2008; Thompson, 2001).

Esa lógica heteronormativa está presente en algunos de los discursos que prevalecen en los criterios de acceso a los tratamientos de reproducción asistida 
o en los discursos de las y los profesionales que componen los equipos de valoración en materia de adopción. En lo que respecta a la reproducción asistida, algunas parejas han sido excluidas de los tratamientos públicos basándose en recientes reformas legislativas que preservan este derecho a parejas heterosexuales. En este sentido, la ley, con el respectivo ideario que la sustenta, puede convertirse en el principal agente vulnerador de los derechos sexuales y reproductivos de las parejas del mismo sexo (Bergmann, 2012; Inhorn y Birembaum-Carmeli, 2008). En cuanto al trato dispensado en las consultas médicas, algunas mujeres han sentido que existe discriminación en comparación a sus homólogas heterosexuales (Tasker, 2013). Los comentarios o la dejadez en la atención son ejemplos de esas conductas discriminatorias a las que se vieron expuestas. Los resultados que arroja la investigación conducida por Záchia y su equipo (2011) revelan que dentro del colectivo médico prevalece una actitud de rechazo a que las parejas de mujeres se sometan a estos tratamientos. En concreto, entre los 224 especialistas consultados en su estudio, un $77 \%$ se mostraba en contra basándose en las consecuencias negativas que tendría para la futura descendencia criarse en una unidad familiar de este tipo.

Por otro lado, los prejuicios y estereotipos hacia las familias del mismo sexo que deciden adoptar también se han constatado en otras investigaciones desarrolladas en países del entorno latinoamericano (Herrera, 2009) y anglosajón (Hicks, 2000; Brooks y Goldberg, 2001; Brown et al., 2009). En este sentido, el miedo a la exclusión del proceso de adopción hace que algunas parejas adopten como personas solteras y oculten su relación de pareja (Gianino, 2008). No obstante, esta pauta de comportamiento no se ha observado en la presente investigación. Las familias adoptivas entrevistadas han coincidido en señalar que la visibilidad y «normalización» del matrimonio entre personas del mismo sexo ha favorecido una percepción más positiva hacia estas parejas como posible madres adoptivas. Esta conexión no se ha encontrado en otras investigaciones sobre la temática.

Otro de los aspectos que deben afrontar estas familias tiene que ver con la importancia conferida al vínculo biológico a la hora de trazar relaciones parentales. En consecuencia, el hecho de que una madre haya experimentado el proceso físico del embarazo, parto y puerperio hace que, en el imaginario y discurso social, se le otorgue una posición privilegiada (Bos, 2013; Donoso, 2013; Sullivan, 2001). La falta de reconocimiento y visibilidad a la que queda expuesta la madre no gestante genera malestar tanto a una madre como a la otra. Ambas identifican que, en base a su personalidad, establecen estrategias de apego diferenciadas con sus hijos e hijas pero que son igualmente importantes 
para su desarrollo personal. La especialización en ciertas actividades (Donoso, 2013) o la búsqueda de tareas alternativas que compensen actos como la lactancia (Goldberg y Perry-Jenkins, 2007) es una táctica recurrente en estas parejas que tratan de superar y combatir la centralidad de lo biológico.

Como principales conclusiones de este trabajo se pueden indicar que la propia construcción heteronormativa y biologicista del parentesco provoca discriminación a quienes se alejan de estos patrones, entre ellas las parejas de mujeres que acceden de forma conjunta a la maternidad. En este sentido, y a pesar de los cambios políticos y culturales que han favorecido la expansión de este modelo familiar, aún prevalecen actitudes prejuiciosas y comportamientos discriminatorios por parte de algunos y algunas profesionales. Las y los trabajadores sociales no pueden permanecer al margen de esta realidad ya que algunas de estas situaciones se producen en su ámbito laboral o en campos próximos a éste. Por este motivo, el trabajo social como disciplina encargada de promover la justicia e igualdad social debe actuar desde dos dimensiones: la preventiva a través de una labor pedagógica sobre lo que significa ser y hacer familia más allá del modelo tradicional heterosexual y, otra de intervención activa en la que se deben denunciar aquellos casos en los que se constaten situaciones discriminatorias basadas en la opción sexual de las progenitoras.

\section{Bibliografía}

BeCK-GERnSHeIM, E. (2002). Reinventing the family: In search of new lifestyles. Cambridge: Polity.

BEN-ARI, A. y LivnI, T. (2006). Motherhood is not a given thing: experiences and constructed meanings of biological and nonbiological lesbian mothers. Sex Roles, 54 (7), 521-531. https://doi.org/10.1007/s11199-006-9016-0

BERGMANN, S. (2012). Cambiar el óvulo manteniendo el fenotipo: la búsqueda de semejanza en la FIV con donación de óvulos transnacional. En E. Pérez y R. Ibáñez (Eds.), Cuerpos y diferencias (pp.55-78). Madrid: Plaza y Valdés.

Bestard, J.; OrobitG, G.; Ribot, J. y SAlazAR, C. (2003). Parentesco y reproducción asistida: cuerpo, persona y relaciones. Barcelona: Universidad de Barcelona.

Bos, H. (2013). Lesbian-mother families formed through donor insemination. En A. Goldberg y K. Allen (Eds.), LGBT-Parent families. Innovations in research and implications for practice (pp. 21-37). New York: Springer. https://doi. org/10.1007/978-1-4614-4556-2_2

BROOKS, D. y GOLDBERG, S. (2001). Gay and lesbian adoptive and foster care placements: Can they meet the needs of waiting children? Social Work, 46 (2), 147-157. https://doi.org/10.1093/sw/46.2.147 
Brown, S.; SMALling, S.; GroZA, V. y RYAN, S. (2009). The experiences of gay men and lesbians in becoming and being adoptive parents. Adoption Quarterly, 12 (3-4), 229-246. https://doi.org/10.1080/10926750903313294

BRYLD, M. (2001). The infertility clinic and the birth of the lesbian: the political debate on assisted reproduction in Denmark. European Journal of Women's Studies, 8 (3), 299-312. https://doi.org/10.1177/135050680100800303

Butler, J. (2006). Deshacer el género. Barcelona: Ediciones Paidós.

Cadoret, A. (2013). Padres como los demás. Parejas gays y lesbianas con hijos. Barcelona: Editorial Gedisa.

Carsten, J. (2004). After kinship. Cambridge: Cambridge University Press.

CUTAS, D. y CHAN, S. (2012). Introduction. Perspectives on private and family life. En: D. Cutas y S. Chan (Eds.), Families. Beyond the nuclear ideal (pp. 1-12). London: Bloomsbury.

Donoso, S. (2013). La familia lesboparental: ¿Reinvención de la familia? (Tesis Doctoral). Barcelona: Universitat de Barcelona.

FINLAY, L. (2014). Engaging phenomenological analysis. Qualitative Research in Psychology, 11 (2), 121-141. https://doi.org/10.1080/14780887.2013.807899

GANONG, L. y GOLEMAN, M. (2014). Qualitative research on family. Journal of Social and Personal Relationships, 31 (4), 451-459. https://doi. org/10.1177/0265407514520828

GiANiNO, M. (2008). Adaptation and transformation: the transition to adoptive parenthood for gay male couples. Journal of GLBT Family Studies, 4 (2), 205243. https://doi.org/10.1080/15504280802096872

GoldberG, A.; Downing, J. y SAUCK, C. (2008). Perceptions of children's parental preferences in lesbian two-mother households. Journal of Marriage and Family, 70, 419-434. https://doi.org/10.1111/j.1741-3737.2008.00491.x

GONZÁlez ECHEvarría, A. ET AL. (2010). Sobre la definición de los dominios transculturales. La antropología del parentesco como teoría sociocultural de la reproducción. Alteridades, 20 (39), 93-106.

Grau, J. (2006). Procreación, género e identidad. Debates actuals sobre el parentesco y la familia en clave transcultural. Barcelona: Ediciones Bellaterra.

Grupo de Interés Centros Reproducción Humana Asistida del Sistema Nacional de Salud (2002). Criterios para la utilización de los recursos del Sistema Nacional de Salud en la aplicación de las técnicas de reproducción humana asistida. Disponible en: http://www.hvn.es/servicios_asistenciales/unidad_de_reproduccion/ficheros/versionfinaldocumentogrupodeinteres.pdf

HAYDEN, C. (2008). Género, genética y generación: reformulación de la biología en el parentesco lésbico. En R. Parkin y L. Stone (Eds.), Antropología del parentesco y de la familia (pp. 621-646). Madrid: Editorial Universitaria Ramón Areces. 
HECKATHORN, D. (1997). Respondent-driven sampling: a new approach to the study of hidden populations. Social Problems, 44 (2), 174-199. http://www. jstor.org/stable/3096941

HICKS, S. (2000). «Good lesbian, bad lesbian...» regulating heterosexuality in fostering and adoption assessments. Child and family social work, 5 (2), 157-168. https://doi.org/10.1046/j.1365-2206.2000.00153.x

HYCNER, R. H. (1985). Some guidelines for the phenomenological analysis of the interview data. Human Studies, 8, 279-303. https://doi.org/10.1007/BF00142995

InHORN, M. y BIRENBAUM-CARMELI, D. (2008). Assisted reproductive technologies and culture change. Annual Review of Anthropology, 37, 177-196. https://doi. org/10.1146/annurev.anthro.37.081407.085230

Johnson, K. (2012). Excluding lesbian and single women? An analysis of U.S. fertility clinic websites. Women's Studies International Forum, 35 (5), 394-402. https://doi.org/10.1016/j.wsif.2012.05.00

LEHR, V. (1999). Queer family values. Debunking the myth of the nuclear family. Philadelpia: Temple University Press.

Peramato, T. (2013). Desigualdad por razón de orientación sexual e identidad de género, homofobia y transfobia. Navarra: Aranzadi.

PiCHARDO, J. I. (2008). Opciones sexuales y nuevos modelos familiares (Tesis Doctoral). Madrid: Universidad Autónoma de Madrid.

RicH, A. (1980). Compulsory Heterosexuality and Lesbian Existence. Signs. Journal of Women in Culture and Society, 5 (4), 631-660. https://doi.org/10.1086/493756

RIVAS, A. M. (2008). Las nuevas formas de vivir en familia: el caso de las familias reconstituidas. Cuadernos de relaciones laborales, 26 (1), 179-202. http://dx.doi. org/10.5209/CRLA.33417

RIVAS, A. M. (2009). Pluriparentalidades y parentescos electivos. Presentación del volumen monográfico. Revista de Antropología Social, 18, 7-19.

RoBinson, B. (2002). Birds do it. Bees do it. So why not single women and lesbians?. Bioethics, 11 (3), 217-227. https://doi.org/0.1111/1467-8519.00060

Rubin, G. (1986). El tráfico de mujeres. Notas sobre la «economía política» del sexo. Revista Nueva Antropología, 8 (30), 95-145.

Ruiz OlabuÉnAga, J. I. (2012). Metodología de la investigación cualitativa. Bilbao: Universidad de Deusto.

STACEY, J. (2005). In the name of the family. Rethinking family values in the Postmodern Age. Boston: Beacon Press.

Stone, L. (2008). Introducción. En R. Parkin y L. Stone (Eds.), Antropología del parentesco y de la familia (pp. 545-558). Madrid: Editorial Universitaria Ramón Areces.

SUlLivan, M. (2001). Alma mater: family «outings» and the making of the modern other mother (MOM). En M. Bernstein y R. Reimann (Eds.), Queer families. 
Queer politics. Challenging culture and the state (pp. 231-253). New York: Columbia University Press.

TASKER, F. (2013). Lesbian and gay parenting post-heterosexual divorce and separation. En A. Goldberg y K. Allen (Eds.), LGBT-Parent families. Innovations in research and implications for practice (pp. 3-20). New York: Springer. https:// doi.org/10.1007/978-1-4614-4556-2_1

THOMPSON, C. (2001). Strategic naturalizing: kinship in an infertility clinic. En S. Franklin y S. Mckinnon (Eds.), Relative Values. Reconfiguring kinship studies (pp. 175-202). Durham: Duke University Press.

Weeks, J.; Heaphy, B. y Donovan, C. (2001). Same sex intimacies. Families of choice and other life experiments. London: Routledge. https://doi. org/10.4324/9780203167168

YANAGISAKO, S. y COLLIER, J. (1999). Toward a unified analysis of gender and kinship. En S. Yanagisako y J. Collier. (Eds.), Gender and kinship. Essays toward a unified analysis (pp.14-50). California: Stanford University Press.

ZÁCHIA, S. et al. (2011). Assisted reproduction: What factors interfere in the professional's decisions? Are single women an issue? BMC Women's Health, 11 (21), 2-10- https://doi.org/10.1186/1472-6874-11-21 
Cita bibliográfica: Beloki-Marañon, U. y Mosteiro-Pascual, A. (2017). Análisis del modelo de vulnerabilidad para la toma de decisiones en el ámbito de los cuidados de las personas mayores [Analysis of the vulnerability model for decision making related to the care of the elderly]. Alternativas. Cuadernos de Trabajo Social, $24,29-42$. https://doi.org/10.14198/ALTERN2017.24.02

\title{
ANÁLISIS DEL MODELO DE VULNERABILIDAD PARA LA TOMA DE DECISIONES EN EL ÁMBITO DE LOS CUIDADOS DE LAS PERSONAS MAYORES ${ }^{1}$
}

\section{ANALYSIS OF THE VULNERABILITY MODEL FOR DECISION MAKING RELATED TO ELDERLY CARE}

\author{
USUE BELOKI MARAÑÓN \\ Universidad de Deusto. \\ usue.beloki@deusto.es \\ Amaia Mosteiro Pascual \\ Universidad de Deusto. \\ amaia.mosteiro@deusto.es
}

\section{Resumen}

Este artículo analiza la validez de la vulnerabilidad como modelo para la toma de decisiones relativas a la situación personal y socio-familiar de la persona mayor necesitada de cuidados. La adecuación del modelo responde a la centralidad del elemento riesgo en la toma de decisiones, a la naturaleza del cuidado y al respeto de los principios que inspiran los derechos de las personas mayores vulnerables. El concepto de vulnerabilidad entronca con una nueva concepción en la que la vulnerabilidad se fundamenta, no sólo en la pérdida de la autonomía, sino en la susceptibilidad de quiebra de los derechos de la dignidad e integridad en la vida cotidiana; en consonancia con una concepción humana existencialmente diversa y vulnerable. La incorporación de este enfoque al Derecho de la capacidad de la persona mayor y regulación de los servicios sociales, exigirá su toma en consideración en los informes y diagnósticos de Trabajo Social.

Palabras clave: Vulnerabilidad, personas mayores, toma de decisiones, cuidados.

\section{Extended abstract}

\section{Introduction}

The analysis in this article focuses on the validity of vulnerability as a model for decision-making related to

\begin{abstract}
This article analyses the validity of vulnerability as a model for decision-making regarding the personal, family and social situation of the elderly in need of care. The adequacy of the model meets the requirements of risk as a core element in decision making and those coming from the nature of care and from the respect for the principles that inspire the rights of vulnerable older people. The concept of vulnerability connects with a new conception of vulnerability, which is not only based on the loss of autonomy, but also on the susceptibility of breaking the rights of dignity and integrity in daily life; in accordance with a new conception of humanity as existentially diverse and vulnerable. The inclusion of this approach into the law of the capacity of the elderly and into the regulation of social services will require taking the analysis of vulnerability into consideration in social work diagnosis and reports.
\end{abstract}

Key words: Vulnerability, the elderly, decision making, care.

personal and socio-family situations of elderly people who require care.

The idea of risk is the key element of analysis in the vulnerability approach, which has historically been applied in studies on poverty, natural disasters and,

Recibido: 11/04/2017

Aceptado: 27/07/2017

Publicado: 21/12/2017

1. Este artículo ha sido presentado como ponencia en el Grupo de Trabajo 9.2. "Cuidados en la sociedad del riesgo: vulnerabilidad, ciudadanía y justicia", del VI Congreso de la Red Española de Políticas Sociales, celebrado en Sevilla los días 16 y 17 de febrero de 2017. 
later, in the realm of gerontology. Furthermore, the risk concept forms the core of the decision-making theory.

\section{Methods}

The starting hypothesis is as follows: given the convergence of the risk concept in the vulnerability approach and in the field of decision-making, analysis of vulnerability in older people constitutes a correct model for decision-making related to their care. The vulnerability model also addresses the demands arising from the type of care in the home and observance of the inspiring principles of laws that protect the elderly.

The article, which is theoretical, identifies the elements of the vulnerability model in elderly care and analyses the vulnerability concept from the perspective of older people's rights.

\section{Findings and discussion}

a. Components of vulnerability in older people who require care.

The vulnerability which affects the elderly (consisting of threats, exposure, possibility and extent of harm and confrontation) and the possibility of taking action in this area (Schröder-Butterfil \& Marianti, 2006; Schröder-Butterfill, 2013), are determined by their physical and mental health, cognitive capacity, social networks and support, material resources, housing, knowledge and experience, availability of social services as well as their individual personality, expectations and personal motivation. The following vulnerability conditions are important: declining health -including memory impairment-, limited mobility and capacity to carry out activities, pain and insecurity (Feito Grande, 2007; Delgado Rodríguez, 2012). Other key vulnerability conditions include a lasting internalised feeling of frailness, helplessness and personal insecurity (Filgueira, 2001; Busso, 2001; Cardona, 2001, Castel, 2014), associated with the risk concept and the perception of not being able to control their own destiny.

In vulnerable older people, this psychological condition commonly underlies the idea of vulnerability itself (Clement $\&$ Bolduc, 2004) that arises not only from frailness or dependence but also due to stressful events. These may be one-off or unexpected occurrences like the loss of the partner, falls and household accidents or hospitalization. Vulnerable older people are more exposed to critical events, which, together with greater frailness and dependence, hinder resilience in traumatic situations, given that such moments increase their mistrust and insecurity concerning the future (Ipsos Mori, 2014).

As for coping mechanisms, care in the home is characterised by informal and flexible strategies that are constantly adapted, with a strong relational component. This is consistent with the vulnerability felt by older people when facing difficulties.

\section{b) Vulnerability as overcoming lack of autonomy}

The vulnerability concept thus fits in a conception of the need for care that goes beyond the liberal idea of autonomy and is linked to man's anthropological vulnerability in which specific conditions of frailness and helplessness are also recognised (Ricoeur, 2008-a; Delgado Rodríguez, 2012; Martín Palomo, 2016).

The vulnerability concept is connected to a new idea in which difficulties are not only based on one's lack of autonomy but on how easily the right to dignity and integrity in daily life can be violated. This is in line with an existentially diverse and vulnerable view of man (Rendtorff, 2002; Palacios Rizzo, 2008; Gordillo Álvarez-Valdés, 2008; Requena Meana, 2008; Guzmán Castillo, Toboso Martín, \& Romañach Cabrero, 2010; Bermejo, 2014).

\section{c. Decisions related to naturalistic and shared types of care}

Decisions on care during ageing are characterised by having a direct impact on older people's lives, including emotional and evaluative elements and adopting to the daily socio-family context (Agrela Romero, Martín Palomo, \& Langa Rosado, 2010; Delgado Rodríguez, 2012; Ramos Ponzón, 2012; Bosch Fontcuberta, 2012). They refer to aspects which are familiar, understandable and manageable for older people (Salthouse, 1996; Wood \& Tanius, 2008; Foster et al., 2008; Craig \& Higgs, 2012). These decisions should therefore be based on the naturalistic theory (Klein, 1999) (Zsambok \& Klein, 1997) and shared mind (Epstein, 2013; Elwyn, 2012).

In contrast to a contractual relationship model which centres on informed consent and seeks to avoid intrusion in older people's lives and rights, shared naturalistic-based decisions are made with a focus on person-centred helping relationships. This is a constant negotiation process which includes reflection on the tasks and risks of daily personal and home life. Control and independence-related aspects are discussed and agreed on. Thus, a reflection of the logic that older people apply in their decisions on risks and difficulties is formed, based on normalisation and continuous adaptation strategies (Craig \& Higgs, 2012; Taylor $\&$ McKeown, 2013). In this manner, the elderly's empowerment and satisfaction are fostered while also promoting the co-responsibility of those taking part in the decision-making (Ipsos Mori, 2014).

\section{Conclusions}

Assessment of decision-making capacity in legislation which affects the elderly, as well as that which regulates social services in Spain, continues to be based on a rational idea of autonomy, which is the traditional vision found in Civil Law. In contrast to other regulations in which the vulnerability focus underpins the legal system which protects the elderly, the Spanish legal system continues to address the subject's individual autonomy and rational capacity for informed consent.

It would be advisable that the reform on capacity (Law/26/2015 of 28 July, modification of the system to protect children and adolescents) prompt changes in the determination of the elderly's legal decision-making capacity and go beyond a strictly cognitive vision to centre on assessing decision-making capacity related to daily care as persons understanding their vulnerability; in other words, a focus on older persons' capacity and will to overcome problems or defend themselves from threats in the environment as well as the existence or availability of the means to face them. These aspects should form part of the social diagnoses and reports issued from the discipline of Social Work with this aim in mind. Shared decision-making should also be considered if older people, regardless of their cognitive impairments, are to exercise their right to take part in decisions that affect them.

Key words: Vulnerability, the elderly, decision making, care, Social Work. 


\section{Introducción}

La vulnerabilidad en el ámbito de la gerontología se aborda prioritariamente desde dos enfoques: un primer enfoque asociado los derechos humanos de las personas mayores como grupo que en reiteradas ocasiones es objeto de una desigualdad material y de una discriminación indirecta; y un segundo enfoque ligado a la idea del riesgo, que considera a las personas mayores como un colectivo necesitado de especial cuidado y protección por su mayor susceptibilidad a sufrir un daño que resulta de diversas amenazas (Barranco Avilés, 2014).

Este segundo enfoque encuentra sus raíces en el concepto y modelos de la vulnerabilidad frente a la pobreza (Chambers, 1989; 1995) y ante los desastres naturales (Blaikie et al., 1994). Su desarrollo posterior persigue capturar la diversidad y la complejidad de los riesgos contemporáneos junto con la variedad de formas de desprotección, inseguridad e incertidumbre (Busso, 2002). Hoy cabe afirmar que los grupos vulnerables pueden ser tantos como riesgos a los que están expuestos (Sánchez González et al., 2012) y su análisis permite prevenir o minimizar los daños a través de la toma de las decisiones más adecuadas.

El enfoque de la vulnerabilidad se aplica en la gerontología posterior a los noventa (Tullman \& Chang, 1999; Schröder-Butterfil \& Marianti, 2006; Andrew et al., 2008; Bilotta et al., 2010; Brodiez-Dolino, 2013; 2014), bien para analizar las condiciones sociales de un grupo que se enfrenta a los riesgos derivados del envejecimiento, bien desde el nivel micro o meso-social de la persona mayor, como sujeto que vive su vulnerabilidad de forma personal y circunstancial (Brodiez-Dolino, 2014; 2016; Clement \& Bolduc, 2004; Hall, 2014; Naik et al., 2010;). Afirma en este sentido Martín Palomo (2016: 167) que se impone «analizar el cuidado y las vulnerabilidades desde perspectivas micro, para conocer cómo las formas diversas de vulnerabilidad se manifiestan en hombres y mujeres concretos que tienen sus problemas concretos».

\section{El concepto de vulnerabilidad y sus componentes en el colectivo de personas mayores}

La vulnerabilidad en el segundo de los enfoques citado se define por la exposición de una persona, familia, grupo o comunidad a uno o varios riesgos determinados, así como por la previsible incapacidad de respuesta para hacer frente a los mismos (CEPAL-CELADE, 2002). En síntesis, la vulnerabilidad hace referencia a la factibilidad de que un sujeto, grupo o comunidad se vea afectado por el daño que se deriva de una amenaza (Cardona, 2001). La propia exposición a la amenaza, la incidencia del daño, así como la capacidad de 
anticipación o de respuesta están determinadas por los condicionantes estructurales, sociales y personales (Bello Sánchez \& González Rego, 2012). De esta forma, la vulnerabilidad de quien está en ella se presenta como un proceso multidimensional en el que confluyen todos los elementos -factores internos y externos-, que dan lugar al riesgo o a la probabilidad del daño en un tiempo y un espacio determinado (Busso, 2001). Como señala Katzman (2000: 281), «las situaciones de vulnerabilidad suelen desencadenar sinergias negativas que tienden a un agravamiento progresivo».

Este carácter multidimensional y acumulativo exige tener en cuenta no sólo los componentes de la vulnerabilidad (amenaza, grado de exposición, posibilidad de generación de un daño, magnitud del mismo y capacidad de afrontamiento), sino también las relaciones que se establecen entre ellos. La virtualidad del enfoque de la vulnerabilidad radica, precisamente, en la posibilidad de actuar en cada uno de esos niveles: sobre la propia existencia de la amenaza, sobre la exposición de la persona, hogar o comunidad, sobre su capacidad de afrontamiento y sobre la eventualidad del daño (Schröder-Butterfil \& Marianti, 2006; Schröder-Butterfill, 2013).

Figura 1. Un marco para comprender la vulnerabilidad

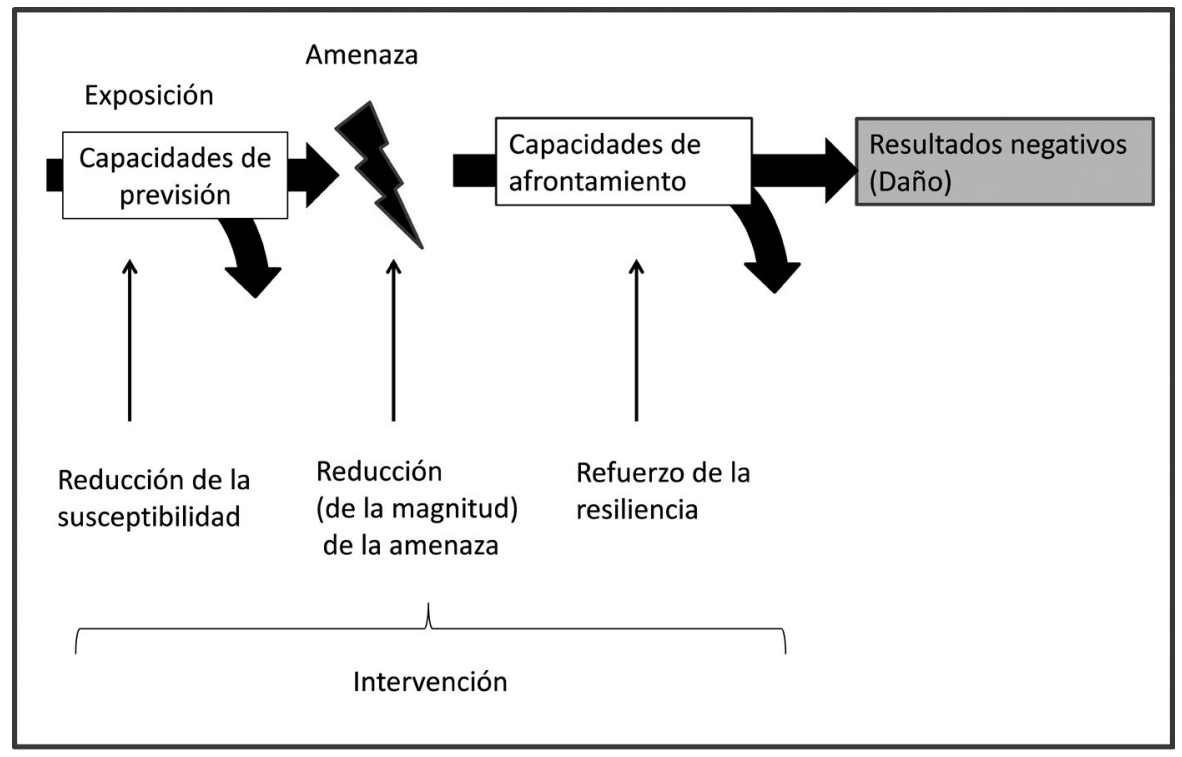

Fuente: Adaptado de Schröder-Butterfill (2013) 
La vulnerabilidad adquiere distintas formas: así, la debilidad interna o fragilidad del individuo, su indefensión ante el entorno, el desamparo institucional o la incapacidad e inseguridad a la hora de aprovechar oportunidades y pensar estrategias para la mejora del bienestar (Busso, 2001). En el caso de las personas mayores su vulnerabilidad depende de un amplio espectro de factores de salud física, mental, capacidades cognitivas, redes y apoyo social, recursos materiales, vivienda, conocimiento y experiencia, disponibilidad de servicios sociales, personalidad individual y las expectativas y motivaciones personales. Entre ellos se apuntan como condiciones de vulnerabilidad más destacables las siguientes: el declive de la salud -incluido el deterioro de la memoria-, las limitaciones de la movilidad y de la capacidad de realización de actividades, el dolor, y la inseguridad (Feito Grande, 2007; Delgado Rodríguez, 2012).

El enfoque incorpora la vertiente subjetiva de la vulnerabilidad, es decir, el sentimiento de inseguridad que resulta de las condiciones de la vejez y que tiene un fuerte impacto en el bienestar percibido de la persona mayor. La vulnerabilidad va más allá de la percepción puntual de las amenazas y se convierte en un sentimiento permanente en la vida de la persona mayor, vinculado a sensaciones de fragilidad, indefensión e inseguridad personal que se mantienen en el tiempo (Filgueira, 2001; Busso, 2001; Castel, 2014), y que pueden llegar incluso a afectar a la identidad personal (Cardona, 2001). Este sentimiento surge de la incertidumbre asociada al propio concepto de riesgo (Clement \& Bolduc, 2004) - de la indeterminación de que la amenaza finalmente tenga lugar o de que el daño se produzca-, así como de la percepción de la eventual incapacidad de controlar el propio destino. En el caso de las personas mayores vulnerables, a este estado psicológico subyacente a la noción misma de vulnerabilidad se añade el que resulta del impacto de acontecimientos estresantes imprevistos, tales como la pérdida de personas significativas, las caídas y accidentes domésticos, y los ingresos hospitalarios. La persona mayor vulnerable está más expuesta a acontecimientos críticos, tiene menos reservas físicas (fragilidad) y funcionales (dependencia) para responder a una crisis o trauma (resiliencia), y éstos constituyen habitualmente puntos de inflexión en relación con la confianza y seguridad en la situación personal futura (Ipsos Mori, 2014).

\section{Los activos relacionales de las personas mayores para hacer frente a la vulnerabilidad}

El estudio de la vulnerabilidad incluye el análisis del manejo de los recursos y de las estrategias que utilizan las comunidades, familias y personas para enfrentar los efectos del riesgo (Pizarro, 2001). Los colectivos vulnerables ponen en 
marcha sus propias estrategias en función de su situación particular y de sus convicciones. El grupo de personas mayores afronta los riesgos derivados de la vejez recurriendo a los activos personales, a estrategias familiares y comunitarias de cuidado informal, así como a los servicios organizados públicos o privados. Señala Moreno en referencia a los activos informales que, aunque parte de ellos dependan muchas veces del voluntarismo y sean fuente en último término de desigualdad, se caracterizan por una inmediatez y flexibilidad que constituye un gran alivio para quien los utiliza (Moreno, 2001).

El enfoque pone así de relieve el carácter relacional del afrontamiento de las dificultades durante el envejecimiento. Ello supone la superación de una concepción liberal del concepto de autonomía, que había centrado su atención en la garantía de la libertad, independencia y espacio vital libre de injerencias externas (Delgado Rodríguez, 2012) que al mismo tiempo, sin embargo, había permanecido ignorante de la influencia del contexto en la situación de vulnerabilidad.

El modelo de la vulnerabilidad parte de una vulnerabilidad antropológica - «entendida como una condición de fragilidad propia e intrínseca al ser humano, por su ser biológico y psíquico»-, que puede verse modulada por las circunstancias personales o por el propio contexto o dimensión social, que repercuten en la susceptibilidad al daño y en la consecución de la mayor autonomía y calidad de vida (Feito Grande, 2007: 8). Aunque el enfoque admite que el ser humano es esencialmente vulnerable e interdependiente, reconoce que existen personas con mayor fragilidad, indefensión y vulnerabilidad (Martín Palomo, 2016). Se incorpora así el concepto de autonomía relacional como medio para alcanzar la autonomía personal (Ricoeur, 2008-a; Delgado Rodríguez, 2012), meta que además se comparte con otros principios como la dignidad o la integridad (Gordillo Álvarez-Valdés, 2008; Rendtorff, 2002; Requena Meana, 2008), en consonancia con la concepción del vulnerable como la de una persona con «otra manera de ser en el mundo» (Ricoeur, 2008-b: 181). La dignidad deja de estar concebida como un atributo consustancial a la condición de autonomía y racionalidad, como un derecho humano elaborado sobre la capacidad de razonar, sentir o comunicar y se predica de la persona que «es» (Bermejo, 2014; Guzmán Castillo, Toboso Martín, \& Romañach Cabrero, 2010; Palacios Rizzo, 2008).

\section{Vulnerabilidad, toma de decisiones compartida y cuidados cotidianos}

En el enfoque de la vulnerabilidad la persona mayor necesitada de cuidados deja de ser objeto de clasificaciones dicotómicas o categorizadas de capacidad o incapacidad, o de autonomía o dependencia, y pasa a ser considerada como 
una persona con unas condiciones y en un contexto que le hace vulnerable a una disminución de su bienestar. Esta concepción se traslada en términos prácticos al ámbito de la toma de decisiones relativas a la persona mayor, en las que ocupan un lugar central las relativas al cuidado.

Las decisiones sobre los cuidados durante el envejecimiento tienen un impacto directo en la vida de la persona mayor, incorporan elementos emocionales, valorativos y se refieren a aspectos que le resultan cercanos, comprensibles y en cierto sentido manejables (Salthouse, 1996; Wood \& Tanius, 2008; Foster et al., 2008; Craig \& Higgs, 2012). Bajo este prisma resulta coherente que las decisiones sobre el cuidado -en el sentido de caring about y de caring for (Tronto, 1998) -, se tomen sobre la base de la teoría naturalista de la toma de decisiones (Zsambok \& Klein, 1997) y como parte de un proceso interactivo y compartido entre los distintos agentes implicados (shared mind) (Epstein, 2013; Elwyn, 2012). Las decisiones relativas a los cuidados incorporan componentes emocionales, intuiciones o elementos críticos (whole mind) que junto con el conocimiento profesional forman parte del conocimiento compartido (Levin, 2012; Cardona-Cardona et al., 2017). Por lo que respecta al proceso, se trata de un proceso de comunicación y negociación constante en el que afloran los valores personales, se reflexiona sobre las tareas y riesgos de la vida personal y doméstica cotidiana y se discuten y acuerdan aspectos relativos al control y a la independencia. Ello no es sino un reflejo de la lógica que siguen las personas mayores en sus decisiones sobre los riesgos y dificultades, que está basada en la normalización y en estrategias de adaptación continuas (Craig \& Higgs, 2012; Taylor \& McKeown, 2013).

Frente a un modelo de relación contractual, que tiene como elemento central el consentimiento informado y que persigue la no intromisión en la vida y derechos de la persona mayor, las decisiones compartidas se toman bajo el enfoque de la relación de ayuda centrada en la persona (Delgado Rodríguez, 2012). Se reconoce la autonomía relacional de la persona mayor y se promueve su autogobierno, partiendo de su interdependencia del contexto socio-familiar cotidiano en el afrontamiento de las condiciones de vulnerabilidad (Agrela Romero, Martín Palomo, \& Langa Rosado, 2010) y tomando en consideración el hecho de que las personas significativas son aquellas con las que habitualmente se consultan y reflexionan las decisiones relevantes. (Delgado Rodríguez, 2012; Ramos Ponzón, 2012; Bosch Fontcuberta, 2012).

En un plano práctico, la toma de decisiones compartida permite decidir con la persona mayor vulnerable que lo autorice, la continuidad de su participación en las tareas cotidianas que impliquen un riesgo, o la incorporación, en su caso, de los cambios, ajustes y adaptaciones necesarias. Se trata de elevar las 
expectativas de lo que es posible a los niveles más altos, con el fin de promover el empoderamiento y la satisfacción de la persona mayor, al tiempo que se favorece la corresponsabilidad de quienes participan en la toma de decisiones (Ipsos Mori, 2014).

\section{Discusión: la aplicabilidad del modelo a la toma de decisiones relativas a personas mayores vulnerables con dificultades cognitivas}

La toma de decisiones compartida puede ponerse en cuestión cuando la persona mayor vulnerable tiene sus capacidades cognitivas mermadas y su participación en el proceso de toma de decisiones queda por ello cuestionado ab initio. La consideración de las opiniones, emociones y deseos de esta persona y la participación de familiares o personas allegadas tiene cabida en el principio deontológico de trabajo social de promoción integral de la persona, pero su encaje entraña una mayor dificultad en la regulación actual de los servicios sociales. Cabe afirmar que la normativa de los servicios sociales descansa todavía sobre un concepto racional de la autonomía, de corte cognitivo, que subraya el consentimiento informado propio del Derecho civil tradicional. A diferencia de lo que sucede en el ámbito anglosajón, en el que el enfoque de la vulnerabilidad subyace en el sistema legal protector de las personas mayores (ABA, 2005; 2006; Care Act de 2014 del Reino Unido, secciones 42-7) -posibilidad que es objeto de análisis también en los últimos años en el Derecho civil Francés (Rebourg \& Bourdin, 2014)-, en el ordenamiento jurídico español, permanece todavía un enfoque basado en la autonomía individual y en el consentimiento. La normativa autonómica reguladora de los servicios sociales que se aprueba en la primera década del nuevo milenio incorpora expresamente en su articulado como principio regulador la autonomía de las personas usuarias sobre su propio proyecto vital, así como su derecho a consentir, a renunciar o a adoptar decisiones libres sobre las intervenciones sociales, supeditadas a la legislación civil vigente, en aquellos casos de personas incapacitadas o presuntas incapaces.

La recepción del Convenio sobre los Derechos humanos de las personas con discapacidad, de 13 de diciembre de 2006, operada en el ordenamiento jurídico civil con las últimas reformas relativas a la modificación de la capacidad (Ley 26/2015, de 28 de julio, de modificación del sistema de protección de la infancia y de la adolescencia), sienta las bases para un cambio en la normativa de servicios sociales que supere la tradicional dicotomía entre capacidad e incapacidad. La instauración del sistema de apoyos necesarios para la promoción de la toma de decisiones de la persona con discapacidad, -incluidas las personas mayores-, y la creciente diferenciación de las esferas de la persona a los efectos 
de la valoración de su capacidad, permiten vaticinar un área de desarrollo del trabajo social. La consideración de las habilidades para la vida independiente como área específica de valoración en los procesos de modificación de la capacidad (Instrucción n. ${ }^{\circ}$ 3/2010 de la Fiscalía General del Estado) ubicará al trabajador/a social como un agente clave en la determinación de la capacidad de la persona mayor vulnerable con deterioro cognitivo (Rueda, 2012), así como en la promoción de su autonomía, también en sentido relacional. Procede en estos casos entonces reconstruir lo que fuera su voluntad, preferencias y valores conciliándolos, en la medida de lo posible, con la gestión del riesgo que en el momento actual configura su vulnerabilidad.

\section{Conclusiones}

Como se ha dicho hasta el momento, los cuidados de las personas mayores vulnerables se caracterizan por la confluencia de aspectos sociales y sanitarios, con implicaciones socio-familiares, en los que está presente un estado psicológico de incertidumbre y cuya resolución actual depende de la puesta en marcha de activos, en gran medida informales. Es lugar común que el trabajo social tiene como uno de sus elementos distintivos la libertad e individualidad consideradas en el contexto social de la persona. El respeto de la voluntad libremente formada de la persona mayor y la promoción de su red de apoyo, con la toma en consideración de sus opiniones, forman parte de los principios y fundamentos de la disciplina.

Si el modelo de la vulnerabilidad resulta de utilidad para que profesionales, personas mayores y personas significativas identifiquen el riesgo y los activos disponibles y establezcan estrategias conjuntas para su gestión; cuando la persona mayor tiene sus facultades cognitivas mermadas las decisiones relativas a sus cuidados exigen, además, un trabajo social dirigido a identificar y analizar los elementos sociales que permitan valorar la capacidad de esta persona de comprender, asumir y gestionar su propia vulnerabilidad.

Los riesgos que resultan del contexto, la necesidad de recepción de cuidados y la comprensión de la persona mayor de su propia situación constituyen elementos valorativos que habrán de formar parte de los diagnósticos e informes sociales que a tal fin se emiten desde la disciplina del trabajo social. Asimismo, la toma de decisiones compartida habrá de ser considerada si se quiere hacer efectivo el derecho de la persona mayor, -aun con dificultades cognitivas- a participar en las decisiones que le afectan. 


\section{Bibliografía}

ABA. American Bar Association. Commision on Law and Aging \& American Psichological Association. (2005). Assessment of Older Adults with Diminished Capacity: A Handbook for Lawyers. Washington, DC: ABA. American Bar Association \& American Psichological Association. Disponible en: http://www. apa.org/pi/aging/resources/guides/diminished-capacity.pdf

ABA. American Bar Association. Commision on Law and Aging \& American Psichological Association. (2006). Judicial Determination of Capacity of Older Adults in Guardianship Proceedings. Handbook for Judges. Washington DC: American Bar Association \& American Psychological Association. Disponible en: https://www.apa.org/pi/aging/resources/guides/judges-diminished.pdf

Agrela-Romero, B., Martín-Palomo, M. T. y Langa-Rosado, D. (2010). Modelos de provisión de cuidados: género, familias y migraciones. Nuevos retos y configuraciones para las políticas. Alternativas. Cuadernos de Trabajo Social, 17, 9-17. https://doi.org/10.14198/ALTERN2010.17.1

Andrew, M., MitnitsK, A. \& RocKWOOD, K. (2008). Social Vulnerability, Frailty and Mortality in Elderly People. PLoS ONE, 3(5). https://doi.org/10.1371/ journal.pone.0002232

BARRANCO AVILÉs, M. (2014). Derechos humanos y vulnerabilidad. Los ejemplos del sexismo y del edadismo. En M. Barranco Avilés y C. Churruca Muguruza, Vulnerabilidad y protección de los derechos humanos (16-44). Valencia: Tirant lo Blanch.

Bello SÁnChez, W. y González Rego, R. A. (2012). Vulnerabilidad de las personas de la tercera edad ante el riesgo de envejecimiento en Cuba. En C. Ejea Jiménez, D. Sánchez González y J. I. Soledad Suescún, Vulnerabilidad Social. Posicionamientos y ángulos desde geografías diferentes (201-215). Granada: Eirene.

BERMEJO, L. (2014). Estudio sobre la creación de una unidad de calidad de vida en personas con demencia en sus fases iniciales. Madrid: Ministerio de Sanidad y Servicios Sociales e Igualdad. Disponible en: http://www.fundacionreinasofia. es/Lists/Documentacion/Attachments/66/12015_unidad_calidad_vida.pdf

Bilotta, C., Casè, A., Nicolini, P., Mauri, S., Castelli, M. \& Vergani, C. (2010). Social vulnerability, mental health and correlates of frailty in older outpatients living alone in the community in Italy. Ageing and Mental Health, 14(8), 10271036. https://doi.org/10.1080/13607863.2010.508772

Blaikie, P., Cannon, T., Davis, I. \& Wisner, B. (1994). At Risk: Natural Hazards, People Vulnerability and Disasters (1st. ed.). London-New York: Routledge.

BosCh FontCUBERTA, J. M. (2012). La toma de decisiones conjunta en medicina: una difícil asignatura. Revista de atención primaria, 17, 385-387. https://doi. org/10.1016/j.aprim.2012.05.010 
Brodiez-Dolino, A. (2013). Combattre la pauvreté. Vulnérabilités sociales et sanitaries de 1880 à nos jours. Paris: CNRS.

Brodiez-Dolino, A. (2014). La vulnérabilité, entre sanitarie et social. En A. Brodiez-Dolino, I. Von Vueltzingsloewen, B. Eyraud, C. Laval, \& B. Ravon, Vulnérabilités sanitaires et sociales (9-16). Rennes Cedex: Presses Universitaires de Rennes.

Brodiez-Dolino, A. (2016). Le concept de vulnérabilité. Disponible en: www.laviedesidees.fr.

Busso, G. (2001). Vulnerabilidad social: nociones e implicancias de políticas para latinoamérica a inicios del siglo XXI. Seminario Internacional «Las Diferentes expresiones de la Vulnerabilidad Social en América Latina y el Caribe», 20 y 21 de junio. Santiago de Chile: Naciones Unidas. Comisión Económica para América Latina y el Caribe. CEAPAL. Disponible en: http://www.cepal.org/ publicaciones/xml/3/8283/GBusso.pdf

Cardona-Cardona, J. Cuartero-Castañer, M. E. y Campos-Vidal, J.F. (2017). La comprensión de la situación problema en la práctica del Trabajo Social de Casos. Resultados de una investigación. Cuadernos de Trabajo Social, 30(1) 2017: 149-162. http://dx.doi.org/10.5209/CUTS.51735

CARDONA, O. D. (2001). La necesidad de repensar de manera holística los conceptos de vulnerabilidad y riesgo: una crítica y una revisión necesaria para la gestión. Memoria International Work-Conference on Vulnerability in Disaster Theory and Practice. Disponible en: http://www.desenredando.org/public/ articulos/2001/repvuln/RepensarVulnerabilidadyRiesgo-1.0.0.pdf

CASTEL, R. (2014). Los riesgos de exclusión social en un contexto de incertidumbre. Revista Internacional de Sociología (RIS), 72 (Extra 1), 15-24. Procesos de exclusión social en un contexto de incertidumbre). https://doi.org/10.3989/ ris.2013.03.18

CEPAL-CELADE. (2002). Vulnerabilidad sociodemográfica: viejos y nuevos riesgos para comunidades, hogares y personas. Brasilia: CEPAL-CELADE. Naciones Unidas. Disponible en: http://www.cepal.org/publicaciones/xml/4/11674/ LCW3-Vulnerabilidad.pdf

Chambers, R. (1989). Vulnerability, Coping and Policy. IDS Bulletin, 20(2), 1-7. https://doi.org/10.1111/j.1759-5436.1989.mp20002001.x

CHAMBERS, R. (1995). Poverty and livelihoods: whose really counts? Environment and Urbanization, 7(1), 173-204. http://www.archidev.org/IMG/pdf/p173.pdf

Clement, M. \& Bolduc, N. (2004). Regards croisés sur la vulnérabilité. En F. Saillant, M. Clément \& C. Gaucher, Identités, vulnérabilités, communautés (6182). Montréal: Les Éditions Nota Bene.

Craig, G. M. \& HigGS, P. (2012). Risk owners and risk managers: Dealing with the complexity of feeding children with neurodevelopmental disability. 
Negotiating and communicating health risk. Health, Risk \& Society, 14 (7-8), 627-637. https://doi.org/10.1080/13698575.2012.717610

DELGADO RodríGUEZ, J. (2012). Nuevas perspectivas bioéticas: autonomía relacional. Revista de Enfermería, 6 (1), 35-42. Disponible en: http://ene-enfermeria. org/ojs/index.php/ENE/article/view/8/7

Elwyn, G., Frosch, D., Thomson, R., Joseph-Williams, N., Lloyd, A., Kinnersley, P. \& BARry, M. (2012). Shared Decision Making: A Model for Clinical Practice. Journal of General Internal Medicine, 27(10), 1361-1367. https://doi. org/10.1007/s11606-012-2077-6

EPSTEIN, R. M. (2013). Whole mind and shared mind in clinical decision-making. Patient Education and Counseling, 90, 200-206. https://doi.org/10.1016/j. pec.2012.06.035

España. Ley 26/2015, de 28 de julio, de modificación del sistema de protección a la infancia y a la adolescencia. BOE núm. 180, de 29 de julio de 2015. 64544-63613

Feito Grande, L. (2007). Vulnerabilidad. Anales del Sistema Sanitario de Navarra, 30 (3), 7-22. Disponible en https://recyt.fecyt.es/index.php/ASSN/article/ view/2004/1431

FILGUEIRA, C. H. (2001). Estructura de oportunidades y vulnerablidad social aproximaciones conceptuales recientes. Santiago de Chile: CEPAL. Disponible en: http://www.cepal.org/publicaciones/xml/3/8283/cfilgueira.pdf

Fiscalía General del Estado. Instrucción n. ${ }^{\circ}$ 3/2010 sobre la necesaria fundamentación individualizada de las medidas de protección o apoyo en los procedimientos sobre determinación de la capacidad de las personas. http://www.elsindic.com/documentos/586_INSTRUCCION\%203-2010\%20FISCALIA\%20GENERAL\%20 DEL\%20ESTADO.pdf

Foster, S., Cornwell, R., Michael, A. \& Davis, H. (2008). Cognitive Changes across the Life Span. En S. H. (Ed.), Changes in Decision-Making Capacity in Older Adults. Assesment and Intervention. New Jersey: John Wiley \& Sons, Inc.

GORDILlO ÁlVAREZ-VALDÉs, L. (2008). ¿La autonomía, fundamento de la dignidad humana? Cuadernos de Bioética (66), 237-253.

Guzmán Castillo, F., Toboso martín, M. y Romañach Cabrero, J. (2010). Fundamentos éticos para la promoción de la autonomía: hacia una ética de la interdependencia. Alternativas. Cuadernos de Trabajo Social, 17, 45-61. https:// doi.org/10.14198/ALTERN2010.17.03

Hall, M. (2014). Dementia, Autonomy and Guardianship for the Old. En J. H. Charles Foster, The Law and Ethics of Dementia (339-349). Oxford: Hart Publishing.

IPSOS MORI. (2014). Understanding the lives of older people living with frailty. A qualitative investigation. London: Age UK. Disponible en: https://www. ipsos-mori.com/DownloadPublication/1660_sri-ipsos-mori-age-uk-livingwith-frailty-2014.pdf 
KATZMAN, R. (2000). Notas sobre la mediacion de la vulnerabilidad social. Santiago de Chile: CEPAL. Disponible en: http://www.cepal.org/deype/mecovi/docs/ taller5/24.pdf

LEVIN, L. (2012). Towards a revised definition of client collaboration: the knowledge-power-politics triad. Journal of Social Work Practice, 26 (2), 181195. https://doi.org/10.1080/02650533.2010.545121

Martín PAlOMO, M. T. (2016). Cuidado, vulnerabilidad e interdependencias. Madrid: Centro de Estudios Políticos y Constitucionales.

Moreno, L. (2001). Estados del bienestar y «mallas de seguridad». En L. Moreno, Pobreza y exclusión: la «malla de seguridad» en España (17-50). Madrid: Consejo Superior de Investigaciones Científicas.

Naik, A., Kunik, M., Cassidy, K., Nair, J. \& Coverdale, J. (2010). Assessing Safe and Independent Living in Vulnerable Older Adults: Perspectives of Professionals Who Conduct Home Assessments. JABFM- Journal of the American Board of Family Medicine, 23(5), 614-621. https://doi.org/10.3122/ jabfm.2010.05.090065

ONU. Convención de Nueva York, de 13 de diciembre de 2006, sobre los sobre los derechos de las personas con discapacidad. Instrumento de ratificación de 23 de noviembre de 2007. BOE núm. 96, de 21 abril 2008.

Palacios Rizzo, A. (2008). El modelo social de discapacidad: orígenes, caracterización y plasmación en la Convención Internacional sobre los Derechos de las Personas con Discapacidad. Madrid: CERMI.

PiZARRO, R. (2001). La vulnerabilidad social y sus desafíos: una mirada desde América Latina. Santiago de Chile: CEPAL. Disponible en http://repositorio.cepal.org/ bitstream/handle/11362/4762/S0102116_es.pdf

RAMOS PONZÓN, S. (2012). La toma de decisiones compartidas en pacientes con esquizofrenia: cuestiones médicas y éticas. Dilemata, 4 (10), s.p. Disponible en: http://www.dilemata.net/revista/index.php/dilemata/article/view/179/223

REBOURG M. \& BuRdin E. (2014), La vulnérabilité dans l'espace juridique: la situation des personnes du grand âge, en Brodiez Dolino A., Eyraud B., Laval C., Ravon B. et Von Bueltzingsloewen I. (dir.), Vulnérabilités sanitaires et sociales. Approches sociologiques et historiques, Rennes: PUR.

RendTORFF, J. D. (2002). Basic ethical principles in European bioethics and biolaw: autonomy, dignity, integrity and vulnerability. Towards a foundation of bioethics and biolaw. Medicine, Health Care and Philosophy, 5, 235-244. https://doi.org/10.1023/A:1021132602330

REQUENA MEANA, P. (2008). Dignidad y autonomía en la bioética norteamericana. Cuadernos de Bioética (66), 255-270. Disponible en: http://www.bioeticaweb. com/dignidad-y-autonomasa-en-la-bioactica-norteamericana/ 
Reino Unido. Care Act de 2014, secciones 42-7. Obtenido de http:// www.legislation.gov.uk/ukpga/2014/23/part/1/crossheading/ safeguarding-adults-at-risk-of-abuse-or-neglect/enacted.

Ricoeur, P. (2008-a). Autonomía y vulnerabilidad. En P. Ricoeur, Lo Justo II. Estudios, lecturas y ejercicios de ética aplicada (70-87). Madrid: Trotta.

RicoeUR, P. (2008-b). La diferencia entre lo normal y lo patológico como fuente de respeto. En P. Ricoeur, Lo justo II. Estudios, lecturas y ejercicios de ética aplicada (173-183). Madrid: Trotta.

RUEDA EstRADA, J.D. (2012) La incapacitación judicial y el peritaje social. Una visión desde las fundaciones tutelares. Agathos. Atención sociosanitaria y bienestar. 12 (1), 30-41

Salthouse, T. (1996). Commentary: A Cognitive Psychologist's Perspective on the Assessment of Cognitive Competency. En M. Smyer, W. K. Schaie \& M. B. Kapp. New York: Springer Publishing Company.

SCHRÖDER-ButTERFILL, E. \& MARIANTI, R. (2006). A framework for understanding old-age vulnerabilities. Ageing and Society (26), 9-35. https://doi.org/10.1017/ S0144686X05004423

SCHRÖDER-BUTTERFILL, E. (2013). Le concept de vulnérabilité et sa relation a la fragilité. En F. Béland, \& M. (. Hervé, La fragilité des personnes âgées: Définitions, controverses et perspectives d'action (205-228). Rennes. https://doi.org/10.3917/ ehesp.bela.2013.01.0203

TAYLOR, B. J. \& MCKEOWN, C. (2013). Assessing and managing risk with people with physical disabilities: the development of a safety checklist. Health, Risk \& Society, 15(2), 162-175. https://doi.org/10.1080/13698575.2013.764973

Tronto, J. C. (1998). An Ethnic of care. Generations, 22 (3), 15-20.

Tullman, D. \& Chang, B. (1999). Nursing Care of the Elderly as a Vulnerable Population. Nursing clinics of North America, 34(2), 333-334.

Wood, S. \& TANIUS, B. (2008). Impact of Dementia on Decision Making Abilities. En S. H. (Ed.), Changes in Decision-Making Capacity in Older Adults. Assesment and Intervention (91-97). New Jersey: John Wiley \& Sons, Inc.

ZsAmboK, C. \& Klein, G. (1997). Naturalistic decision making. New Yersey: Lawrence Erlbaum publishers. 
Cita bibliográfica: Cazorla Palomo, J. y Parra Ramajo, B. (2017). El cambio en los modelos del trabajo social en salud mental: del modelo rehabilitador al modelo social [Changes on social work models in mental health: from the rehabilitation model to the social model]. Alternativas. Cuadernos de Trabajo Social, 24, 43-54. https://doi.org/10.14198/ ALTERN2017.24.03

\title{
EL CAMBIO EN LOS MODELOS DEL TRABAJO SOCIAL EN SALUD MENTAL: DEL MODELO REHABILITADOR AL MODELO SOCIAL
}

\section{CHANGES ON SOCIAL WORK MODELS IN MENTAL HEALTH: FROM THE REHABILITATION MODEL TO THE SOCIAL MODEL}

\author{
Josep CAZORLa PALOMO \\ Universitat de Barcelona, Facultat d'Educació, Escola de treball social, Barcelona, España \\ josepcazorla@ub.edu \\ BELÉN PARRA RAMAJO \\ Universitat de Barcelona, Facultat d'Educació, Escola de treball social, Barcelona, España \\ belenparra@ub.edu
}

\section{Resumen}

El modelo rehabilitador es en la actualidad el modelo rector de nuestra sociedad y del mundo de la atención de la Salud Mental. Este estándar exige al individuo que se adapte al medio, responsabilizándolo de sus limitaciones y rechazando la diferencia. Desde esta visión se transforma a la persona en un usuario o un consumidor de servicios, reforzándose la discriminación a partir de los ideales establecidos y los mecanismos de control institucionales.

El trabajo social emerge, desde sus inicios, como una disciplina que reconoce las capacidades y las experiencias de las personas de forma singularizada, para facilitar que sean estas las protagonistas de sus relatos de vida. Es por ello, que en el ámbito de la Salud Mental uno de los principales propósitos es la descategorización de la diferencia que perpetua el estigma. Esta concepción se encuentra estrechamente unida al modelo social, perspectiva que concibe toda vida humana con las mismas condiciones de dignidad que el resto de la sociedad, que facilita a las personas con trastornos mentales el reconocimiento como ciudadanos con plenos derechos y deberes dentro de la sociedad en la que habitan.

Palabras clave: Trabajo Social, Salud Mental, Diversidad, Participación, Estigma, Modelo Rehabilitador y Modelo Social.

\begin{abstract}
Currently, the rehabilitation approach is the guiding model in mental health care as well as in society in general. This standard demands individuals to fit in their environment, making them responsible for their limitations and rejecting difference. From that point of view, a person becomes a user or a consumer, which reinforces discrimination through established ideas and mechanisms of institutional control.

From the beginning, social work emerges as a discipline that recognizes individuals' capacities and experiences in a singular way, making it easier for them to star in their life story. For that reason, one of the main goals in mental health care is the decategorization of the difference which perpetuates the stigma. This idea is closely linked to the social approach, which conceives the whole human life in terms of equal dignity conditions, which makes it easier for people with mental disorders to be acknowledged as citizens with full rights and obligations in the society they are living
\end{abstract} in.

Keywords: social work, mental health, diversity, participation, stigma, rehabilitation approach, and social approach. 


\section{EXTENDED ABSTRACT}

Currently, the rehabilitation approach is the guiding model in mental health care as well as in society in general. This standard demands individuals to fit in their environment, making them responsible for their limitations and rejecting difference. From that point of view, a person becomes a user or a consumer, which reinforces discrimination through established ideas and mechanisms of institutional control.

From the beginning, social work emerges as a discipline that recognizes individuals' capacities and experiences in a singular way, making easier for them to star in their life story. For that reason, one of the main goals in mental health care is the decategorization of the difference which perpetuates the stigma. This idea is linked to the social model, which conceives the whole human life in terms of equal dignity conditions, which makes it easier for people with mental disorders to be acknowledged as citizens with full rights and obligations in the society they are living in.

People who suffer from mental disorders have to face numerous challenges. The diagnosis of a mental disorder triggers a succession of consequences that in result will hugely impact and transform the lives of those who are diagnosed as they will endure high quotes of discrimination, stigmatization and psychosocial distress. The biggest challenge they have to face is the fact that they have been linked to categorizations considered mental disorders, also called stigma. Every time that stigma is studied in diagnosed individuals, feelings of shame and being blamed by others appear at the centre of their perception. The damaging effects of stigma spread over all aspects of life of those diagnosed with a mental disorder making it more difficult for them to get a job or housing, for instance, damaging also their self-esteem as a consequence of the internalized stigma.

It is for that reason that, from mental health care, the decategorization of the difference that perpetuates the stigma is one of the main functions of social work. That is why it is important to become aware of the effects of the diagnosis over the professional relationship, in order to preserve singularity, highlighting and counteracting the ways in which stigma is expressed. Social work in mental health care has to be focused on fighting stigma, and on improving the wellbeing and the «social place» through a sense of belonging and self-capability in people diagnosed with mental disorders, working with them to maximize their strengths and minimize social processes that undermine their social identities.

For a long time, the biomedical model has been the main assistance model in mental health care and in social work. This model is based on focusing on the deficit and on a causal understanding of mental disorders, and on the sense of a chronic course of the mental pathology. This perspective focuses its interest on the cure or treatment of the difference, using policies and interventions with the purpose of normalizing the people diagnosed with mental disorders, making them responsible for the difficulties in adapting to the environment they are living in.

Several voices have warned about the incorporation of the biomedical model by social workers, as it has been not thought through and implemented without criteria; a model that also in social work emphasizes the linear process of the intervention, through which the clinical symptoms that lead to a diagnosis are identified in order to provide treatment, help or intervention guiding. As a consequence, the recovery model emerges as a perspective that causes a philosophical and conceptual change, through changing illness and deterioration as attention cores for strengths and capacities. The recovery model is based on the belief that despite the traumatic experiences linked to a mental health diagnosis, it is possible to rebuild a satisfactory and significant life. This philosophy highlights that recovery is about living a hopeful, significant and purposeful life, and it is equivalent to reaching the capacity for transcending symptoms, functional incapacity and social limitations that influence the disorder. It is about recovering the roles as individuals and citizens.

If we examine the recovery model in depth, we will find that the values and principles on which it is based are the same central values of every social work practice. In the recovery model other elements of the social work practice can also be found, such as listening to people, accepting them just the way they are, and trusting their potential. Another confluent element between social work practice and the recovery perspective is participation. The professional social work practice is performed from the principle that it conceives that every individual is able to identify and deal with their own difficulties, an affirmation which is supported by their strengths, capacities, and abilities. Participation facilitates the change of values and attitudes from society, because by competently voicing and sharing their experiences their knowledge is recognized (Corrigan et al., 2001).

In spite of the recovery model's goodwill, there are forms of discrimination and inequality that persist in its basis and that the implementation of the model could not avoid, because in order to measure and range disability and the access to resources, classifications are still being used. This contributes again to a new control mechanism to resources access and subsequently discrimination against those unable to develop a life project within the established ideas (Mulvale y Bartram, 2015). This paradox has favoured the appearance of a new and more critical position on the discipline, which resulted in the introduction of the social model.

The social model, also called mental diversity model, highlights a change in attitude and a political change within the community towards people's limitations and barriers. This philosophical line of thought postulates that everyone has many characteristics that make them unique beings, asking the community to understand that they should neither protect nor separate from these individuals because they are one of us and, therefore, valuable members of the whole humanity.

The social model of mental diversity makes an appeal to social workers to implement a qualitative and political change in the responsibility for the barriers imposed to people diagnosed with a mental disorder. This change may contribute to the recognition of people's experience as an essential element for the understanding of the situation, and for its approach as well (Brea y Gil, 2016). This change entails a professional challenge because it involves the change on the establishment of the role accepted by the person as a service user to the role of expert and ally towards overcoming the biomedical model in mental health care. With this purpose, social workers must facilitate inclusion of diversity in society through relational nets and community work, to promote the improvement in quality of life of those diagnosed with a mental disorder, through palliating the stigma and inequalities that could emerge (Muyor, 2011).

The acceptance of the social model facilitates those who suffer from a mental disorder are recognized as citizens with full rights and duties within the society they live in.

Keywords: social work, mental health, diversity, participation, stigma, recovery model and social model. 


\section{Introducción}

Los principios y valores del trabajo social enuncian el reconocimiento de la diversidad y la participación activa de las personas en los procesos de intervención, como ejes fundamentales que facilitan el cambio. Para ello se propicia el protagonismo de la persona durante todo el proceso, para así alcanzar el control sobre su propia historia y la mejora de la calidad de vida. Para materializar dichos valores es fundamental que los/las trabajadores/as sociales, desde una mirada crítica, sean capaces de romper con las categorizaciones totalizadoras establecidas socialmente, en pro del reconocimiento de la singularidad del individuo como sujeto único (Derrida, 1997). Porque la reducción de las personas a una idea o prejuicio preconcebidos, perpetua la desigualdad mediante el control y la dominación de la diferencia (Idareta, 2013), reduciendo la capacidad de obrar, y el ejercicio de los derechos y deberes como ciudadanos activos, de las personas diagnosticadas con un trastorno mental.

Con el mismo objetivo que conduce a la plena participación, los/las trabajadores/as sociales reconocen las capacidades y la experiencia de aquellas personas que han sido ignoradas y silenciadas por la sociedad y por las organizaciones que ejercen el poder. Es por ello que la relación profesional que se establecerá será simétrica, mediante dicha relación, el/la profesional conocerá a la persona de forma integral, comprendiendo todos los aspectos de su vida, evitando que se conciba como una simple tarea a la que dar una solución (Farah, Ward, Woolner y Tumer, 2011).

Para ello es necesario que los principios éticos en los que se promueven la dignidad, los derechos, el respeto, la privacidad y la confidencialidad de las personas (Banks, 1997), rijan la intervención, en contraposición a la marginación y al aislamiento individual y social, la privación de derechos y la desigualdad, porque todo ello provoca la aparición de sentimientos de miedo, culpa y soledad (Thornicroft, 2007).

Desde esta perspectiva, la práctica de la disciplina se aproxima a las personas diagnosticadas con un trastorno mental en su vertiente individual y emocional, trabaja con ellas y con sus familias para asegurar que sus derechos ciudadanos no sean vulnerados, y para garantizar su acceso a los recursos en situación de igualdad; ofreciendo una relación profesional que les posibilite hacer frente a las consecuencias de la exclusión y la desigualdad (Cazorla, 2014). Requiere también de una necesaria participación en la comunidad que conecte el malestar individual con una conciencia crítica que concrete cambios en el contexto social (Parra; Oriol y Martínez, 2011)

Los/las trabajadores/as sociales que desarrollan su práctica en el ámbito de la salud mental conciben y despliegan las estrategias que contribuyen a 
mejorar el bienestar y el «lugar social» mediante el fomento de la pertenencia y el sentido de capacidad de las personas diagnosticadas con un trastorno mental, trabajan con las personas para maximizar sus fortalezas y minimizar los procesos sociales que devalúan su identidad social.

\section{El impacto del diagnóstico}

Los diagnósticos de trastorno mental desencadenan una serie de consecuencias de gran impacto que transforman las vidas de las personas que los reciben, llegando a padecer elevadas cuotas de discriminación, desigualdad, estigmatización y sufrimiento psicosocial. Las personas con trastornos mentales tienen que hacer frente a múltiples retos, a la recuperación del significado de la vida, a los sentimientos depresivos, a las ideas suicidas, la vergüenza, la pérdida de la capacidad para disfrutar de la vida, la alienación social y el estigma internalizado (Gelkopf, y Roe, 2014; Michaels, López, Rüsch y Corrigan, 2012).

Diversas investigaciones que analizan en profundidad las experiencias de vida de las personas diagnosticadas de trastorno mental coinciden en destacar que en las experiencias cotidianas de estas personas, se incrustaran vivencias de profundo dolor, aislamiento e impotencia, de injusticia y de derrota. Estas experiencias de sufrimiento aumentan, con frecuencia, por la atención recibida desde servicios, o bien coercitivos o paternalistas (Tew, Shul, Slade, Bird, Melton y Le Boutillier, 2012).

Igualmente en un amplio estudio realizado sobre la percepción de las personas con un diagnóstico grave de salud mental, de la desesperación y el estigma internalizado, se muestra la incidencia negativa del diagnóstico en las experiencias personales y sociales (Williams, Almeida y Knyahnytska,2015).

El mayor reto que tienen que superar las personas inscritas en las clasificaciones diagnosticas que enuncian trastornos graves de salud mental es el estigma, entendido este como todas aquellas características indeseables que se asocian a las enfermedades mentales, que se colocan en categorías que identifican la diferencia entre aquellos que las presentan y los que no (Buck, 2013).

Siempre que se investiga sobre el estigma, en la percepción de las personas diagnosticadas, aparecen en el centro las experiencias de vergüenza, y la vivencia de ser culpabilizadas por los demás. Los efectos nocivos del estigma se extienden en todas las dimensiones de la vida de las personas con trastornos mentales, dificultando la obtención de trabajo o de vivienda por ejemplo, dañando también la autoestima como consecuencia de la internalización del estigma. En comparación con otras circunstancias altamente estigmatizadoras, la enfermedad mental es la más estigmatizante, es por ello que se suele denominar «el estigma extremo» (Thornicroft, 2007). 
Como afirma Idareta (2014), los procesos de categorización de problemas y grupos de población se realizan para poder comparar, medir y calcular, pero «tras la categorización, se debe proceder a descategorizar a cada sujeto. Con la categorización se eliminan todas aquellas cualidades que hacen al sujeto singular, sustituyéndolo por una idea que uniformiza a todas ellas» (Idareta, 2014, pp.17-18).Es por ello que las y los trabajadores sociales deben tomar conciencia de los efectos de la utilización del diagnóstico en la relación profesional, para salvaguardar la singularidad y señalar y contrarrestar las formas en que se expresa el estigma (Michaels, López, Rüsch, y Corrigan, 2012).

Los modelos de atención a la salud mental y la práctica del trabajo social. El modelo médico, el modelo de la recuperación y el modelo social

Durante largo tiempo el modelo imperante de atención en la salud mental, y por ende en el trabajo social en salud mental, ha sido el denominado modelo médico. Este se centra en el déficit, y pone el énfasis en una comprensión causal de los trastornos y en el sentido de un curso crónico de la patología mental (Mulvale y Bartram, 2015). El modelo médico o rehabilitador surgió a principios del siglo XX como evolución de su predecesor, el modelo presidencialista, en el que se entendía la diversidad como un castigo divino, para así transformar esta concepción en enfermedades a las cuales no eran necesarias marginar de la sociedad (Palacios y Romañach, 2006). Desde este enfoque se responsabiliza al individuo de las dificultades de la adaptación al medio en el que habita, centrando su interés en la cura o el tratamiento de la diferencia (Victoria, 2013). Así, desde esta concepción, se pone de relieve la diferencia, estableciéndose políticas y actuaciones que tiene como finalidad la normalización de la persona que sufre una enfermedad mental (Asís, 2013), considerándose imprescindible que este colectivo sea rehabilitado como acción necesaria para su reincorporación como parte de la sociedad de pertenencia (Velarde, 2012).

Puesto que la atención centra su interés en la ausencia de salud, esta se torna en un rasgo que infravalora las actitudes de las personas en relación al resto de la sociedad, propiciando una atención social basada frecuentemente en el paternalismo (Toboso y Arnau, 2008). Desde este modelo la atención es eminentemente jerárquica, el profesional es el conocedor de la solución, prioriza la rehabilitación del individuo, para de esta forma fomentar la creación de una identidad como enfermo (Velarde, 2012) sin tener en cuenta la voluntad de la persona, al ser considerada como menos valorada que el resto (Palacios y Romañach, 2006).

Diversos autores han alertado sobre la incorporación no reflexionada y acrítica del modelo médico por parte de los/las trabajadores/as sociales, en los 
últimos tiempos (Beecher, 2009; Bradhaw, Armour y Roseborough, 2007; Hyde, Bowles, y Pawar, 2015; Karban, 2017), modelo que también en el trabajo social enfatiza un proceso lineal de intervención mediante el cual se identifican los síntomas clínicos que conducen a un diagnóstico para proporcionar el tratamiento, la ayuda o guiar la intervención.

Como consecuencia surge el modelo de la recuperación, perspectiva que provoca no solo un cambio filosófico sino también conceptual al cambiar la enfermedad y el deterioro como núcleo de atención, imperante en el modelo biomédico, por las fortalezas y las capacidades. El modelo de la recuperación afirma que a pesar de las experiencias traumáticas asociadas a un diagnóstico de salud mental, se pueden reconstruir vidas satisfactorias y significativas.

La recuperación en salud mental se centra en tres áreas interconectadas: a) potenciación y control sobre la propia vida, b) inclusión social, relaciones interpersonales y reconstrucción de identidades positivas en contra de la discriminación y el estigma, c) esperanza sobre el futuro y encontrar un sentido y un propósito de vida (Tew et al, 2012).

En definitiva, tal y como plantean Williams, Almeida y Knyahnytska (2015), la recuperación consiste en vivir una vida con esperanza, significado y propósito. O como matizan Bergeron-Leclerc y Cormier (2009), la recuperación equivale a lograr la capacidad para transcender los síntomas, la incapacidad funcional y las limitaciones sociales que influyen sobre el trastorno; consiste en la recuperación del rol de sujeto y ciudadano. En la misma línea, importantes estudios como el de Williams, Almeida y Knyahnytska (2015), enuncian que los elementos fundamentales para lograr la recuperación son la sensación de capacitación y el derecho a la participación plena en el entorno social. Estos autores instan al trabajo social en el ámbito de la salud mental a dirigir sus objetivos a la promoción de la plena ciudadanía de las personas diagnosticadas, a trabajar para combatir el estigma. En consonancia, Buck (2013) también señala que una de las principales finalidades del trabajo social en salud mental es la creación de contextos sociales donde las personas diagnosticadas con trastornos mentales puedan encontrar sentido y esperanza después de haber recibido el diagnóstico.

Algunos autores como Bergeron-Leclerc, y Cormier (2009) y Carter, Pagliano, Francis y Thorner (2016) sustentan que los valores y principios del modelo de la recuperación son los mismos que los valores centrales de toda práctica del trabajo social. Enuncian que las intervenciones de trabajo social preservan elementos definitorios que también son propios, y así han sido enunciados, como constituyentes del modelo de la recuperación. Concretamente, elementos tales como la escucha, la aceptación de la persona tal como es, 
la creencia en su potencial, se identifican como factores que contribuyen al éxito de la intervención en el campo de la salud mental (Bergeron-Leclerc y Cormier 2009). Otro de los elementos señalados, confluente entre la práctica del trabajo social y la perspectiva de la recuperación es la participación. La práctica profesional del trabajo social se realiza desde el principio mediante el que concibe a todo individuo como capaz para identificar y abordar sus propias dificultades, afirmación apoyada en las fortalezas, las capacidades y las habilidades de la persona. La participación posibilita el cambio de actitudes y de valores en la sociedad, puesto que al dar la voz con competencia, y compartir experiencias se reconoce el conocimiento de todas las personas (Corrigan et al., 2001). De forma similar, la literatura sobre el modelo de la recuperación resalta que el reconocimiento de toda experiencia particular facilita el camino hacia la recuperación, mediante la participación de las personas diagnosticadas con un trastorno mental en sus procesos, como expertos y como fuente de conocimiento, hecho que fomenta la autonomía y genera el sentimiento de dignidad (Bradhaw, Armour y Roseborough, 2007).

Sin embargo, a pesar de la buena voluntad que acoge el modelo de la recuperación, en sus fundamentos persisten formas de discriminación y desigualdad que la aplicación del modelo no ha podido evitar, ya que se continua utilizando clasificaciones para medir y graduar la discapacidad y el acceso a los recursos, cuestión que de nuevo genera mecanismos de control y acceso a los recursos, y por lo tanto de discriminación de las personas que no hayan podido desarrollar un proyecto vital que siga los ideales marcados (Mulvale y Bartram, 2015). Esta paradoja ha facilitado la emergencia de posturas más críticas en la disciplina, que han dado lugar a la emergencia del denominado modelo social, que se exporta del movimiento basado en la vida independiente, nacido en Estados Unidos en el campo de la diversidad funcional (Aullé, 2003), que concibe toda vida humana con las mismas condiciones de dignidad que el resto. El modelo social, llamado también de la diversidad mental, apunta a un cambio actitudinal y político de las comunidades hacia las limitaciones y barreras de las personas. Desde esta perspectiva filosófica se postula que todas las personas poseen una serie de características que las convierten en seres únicos, interpelando a la comunidad a entender que no es necesario protegerse o apartarse de ese individuo, ya que es uno más de nosotros y, por tanto, una parte del conjunto valioso de la humanidad.

Por ello, tal y como enuncia Sen (1988), no es concebible un único tipo de comprensión del mundo y de las personas, ya que en la misma idea de igualdad existe la desigualdad, por lo que se prima la elección individual de cada persona a ser y a poder escoger ser diferente, y que esta libertad de elección se 
erija en derechos que puedan ser atendidos. Desde este pensamiento, el trabajo social se responsabiliza de la aceptación de la diferencia, lo que propiciará la participación dignificada de la persona diagnosticada con un trastorno mental dentro de la sociedad como un ciudadano más.

\section{Retos en la práctica del trabajo social}

El modelo social de la diversidad mental invoca a los/las profesionales del trabajo social a un cambio cualitativo y político en relación a la responsabilidad sobre las barreras que se imponen a las personas diagnosticadas con un trastorno mental. Este giro propiciará el reconocimiento de la experiencia de las personas como elemento imprescindible para la comprensión y el conocimiento de la situación, así como para su abordaje (Brea y Gil, 2016).El cambio supone un reto profesional ya que implica que se modifique el hasta ahora asumido papel de la persona como usuaria del servicio, por el de experto y aliado, superando el modelo biomédico de la salud mental. Es por ello que en su práctica, los/las trabajadores/as sociales han de favorecer la inclusión de la diversidad en la sociedad mediante las redes relacionales y el trabajo comunitario, para propiciar una mejora en la calidad de vida de las personas diagnosticadas con un trastorno mental, mediante la paliación de los estigmas y las desigualdades que se pueden generar (Muyor, 2011).

La creación y/o el mantenimiento de relaciones sociales propician en los sujetos la capacidad de asumir su protagonismo social, de dignificar sus relatos vivenciales y de crear nuevas perspectivas relacionales más allá de la verticalidad institucional (Saleebey, 2000).Siendo necesario que las personas diagnosticadas con un trastorno mental puedan compartir experiencias y espacios con otras personas con el objetivo de romper con las limitaciones y barreras personales, mediante la inversión en la comunidad (Brea y Gil, 2016). Esta práctica se basa en los principios y valores intrínsecos del trabajo social: el reconcomiendo de las capacidades, de las habilidades, y del conocimiento de las propias experiencias (Sebastin, 2014).Requiere también de una profunda implicación subjetiva en la aproximación a las personas, a sus limitaciones y/o a sus barreras mediante el reconociendo de su singularidad y dignidad, lo que implica el reconocimiento del otro como un individuo único y valioso para la sociedad en la que habita. Para lograr este propósito es necesario comprometer a los estamentos políticos en el mantenimiento de las personas en su comunidad, y transmitir el valor de la diversidad dentro de la misma. Olvidando viejos dogmas, en los que la suma de las demandas y situaciones desdibujaban a las personas solicitantes de atención (Breton, 1994; Lietz, 2007). 
Demanda también la interpelación a la comunidad para un cambio en la concepción de la responsabilidad de la adaptación a la comunidad como un hecho individual, para que pueda emerger la comprensión de las limitaciones y de las barreras como una cuestión política. Desde esta perspectiva se sustenta la idea de las personas con diversidad como una parte imprescindible y valiosa de la composición de la comunidad de referencia, manteniendo su capacidad en la toma de decisiones relacionadas con su vida. Se puede afirmar que la praxis del/la trabajador/a social para las personas diagnosticadas con un trastorno mental es central para la adquisición de confianza y en la autonomía sobre los propios procesos.

Es responsabilidad del trabajo social el reconocimiento de los conocimientos y de las experiencias vivenciales de las personas silenciadas por su diversidad, para lograr que la práctica del trabajo social devenga una plataforma que facilite la creación de nuevas instituciones de orden simbólico y formal, que amplíen el diálogo junto con los nuevos actores. La reflexión y el control en las actuaciones de las entidades políticas, y la ejecución de las directrices en salud mental se han de realizar desde el reconocimiento de la diversidad como un hecho enriquecedor para la sociedad de pertenencia.

\section{Referencias bibliográficas}

AlluÉ, M. (2003). Discapacitados. La reivindicación de la igualdad en la diferencia. Barcelona: Ediciones Bellaterra.

Asís, R. (2013). Sobre el modelo social de la discapacidad: Críticas y éxito. Papeles el tiempo de los derechos, 1, 1-12.

BANKS, S. (1997). Ética y valores en el Trabajo Social. Barcelona: Paidós.

BEECHER, B. (2009). The medical model, mental health practitioners, and individuals with schizophrenia and their families. Journal of Social Work Practice, 23(1), 9-20. http://dx.doi.org/10.1080/02650530902723282

BERGERON-LeCLERC, C. \& CORMIER, C. (2009). Les ingrédients magiques de la relation d'aide: Une exploration des facteurs à l'origine du succès de l'intervention dans le champ de la santé mentale. Service social 55 (1), 1-16. https://doi. org/10.7202/029486ar

BradHAW, W., ARmour, M.P. \& Roseborough, D. (2007). Finding a place in the world the experience of recovery from severe mental illness. Qualitative Social Work, 6(1), 27-47. https://doi.org/10.1177/1473325007074164

BREA, J. y GIL, H. (2016). Estigma y salud mental. Una reflexión desde el trabajo social. Trabajo Social Hoy, 78, 95-112. http://dx.doi.org/10.12960/ TSH.2016.0012 
BRETON, M. (1994). On the meaning of empowerment and empowerment-oriented social work. Social Work with Groups, 17(3), 23-27. http://dx.doi.org/10.1300/ J009v17n03_03

BUCK, J. (2013). Perceived stigma and societal discrimination in individuals with mental illness: Understanding the importance of a sense of community. Social Sciences Capstone Projects. Paper 22 http://commons.pacificu.edu/cgi/viewcontent. cgi?article $=1018$ E context $=$ cassoc

Carter, M.A., Pagliano, P.; Francis, A. \& Thorne, M. (2016). Australian University Students and Mental Health: Viewpoints from the literature. International Journal of Innovation, Creativity and Change, 3(3), 1-25 www. ijicc.net.

CASAl, J. C. (2010). Derechos y participación de las personas con diversidad mental. Cuadernos de Trabajo social, 23, 301-321.

CAzorla, J. (2014). Las relaciones sociales en Salud Mental: Estudio sobre la percepción del impacto de las relaciones sociales en la mejora de la autonomía de las personas con diversidad mental y la relación con el Trabajo Social. Alternativas. Cuadernos de Trabajo Social, 21,161-176. https://doi.org/10.14198/ ALTERN2014.21.08

Corrigan, P.W; River, L.P.; Lundin, R.L.; PenN, D.L.; UPhOFF-WasowsKi, K.; Campion, J.; Mathisen, J.; Gagnon, C.; Bergman.; Goldstein. \& Kubiak, M.A. (2001). Three strategies for changing attributions. Schizophrenia Bulletin, 27(2), 187-195. https://doi.org/10.1093/oxfordjournals.schbul.a006865

DERRIDA, J. (1997). El monolingüismo del otro, o la prótesis de origen. Buenos Aires: Manantial.

Dominelli, L. (1996). Deprofessionalizing Social Work: Anti-Oppressive Practice, Competencias and Postmodernism. British Journal of Social Work, 26(2), 153175. https://doi.org/10.1093/oxfordjournals.bjsw.a011077

Gelkorpf, M. \& RoE, D. (2014). Evaluating Outcome Domains Assessing Caregivers of Individuals with Mental Illes: A Review. Family process, 53(1), 150-174. https://doi.org/10.1111/famp.12056

Hyde, B., BOWLES, W. \& PAWAR, M. (2015). 'We're Still in There'-Consumer Voices on Mental Health Inpatient Care: Social Work Research Highlighting Lessons for Recovery Practice. British Journal of Social Work, 45(1), i62-i78. https:// doi.org/10.1093/bjsw/bcv093

IDARETA, F. (2013). E. Lévinas y el Trabajo Social: Más allá que de Jonia a Jena. Daímon. Revista Internacional de Filosofía, 58, 19-32.

IDARETA, F. (2014). Propuesta para la medición de la violencia al usuario en el trabajo social desde las perspectivas de E. Lévinas y J. Derrida. Portularia, XIV (1), 15-26. https://doi.org/10.5218/prts.2014.0002 
KARBAN, K. (2017). Developing a Health Inequalities Approach for Mental Health Social Work. British Journal of Social Work, 47 (3), 885-992. https://doi. org/10.1093/bjsw/bcw098

LiETZ, C.A. (2007). Strengths-Based Group Practice: Three Case Studies. Social Work with Groups, 30(2), 73-87. https://doi.org/10.1300/J009v30n02_07

Michaels, P.J.; LÓPEZ, M.; RÜSCH, N. \& CORRIGAN, P. W. (2012). Constructs and concepts comprising the stigma of mental illness. Psychology, Society $\varepsilon$ Education, 4(2), 183-194. https://doi.org/10.25115/psye.v4i2.490

Mulvale, G. \& BARTRAM, M. (2015). No More «Us» and «Them»: Integrating Recovery and Well-Being into a Conceptual Model for Mental Health Policy. Canadian Journal of Community Mental Health, 34(4), 31-67. https://doi. org/10.7870/cjcmh-2015-010

MuYor, J. (2011). La (con) ciencia del Trabajo Social en la discapacidad: Hacia un modelo de intervención social basado en derechos. Documentos de trabajo Social, 49, 9-33.

Palacios, A. y RomañaCH, J. (2006). El modelo de la diversidad: La bioética y los derechos humanos como herramientas para alcanzar la plena dignidad en la diversidad funcional. Madrid: Diversitas.

PAlacios, A. (2008). El modelo social de discapacidad: orígenes, caracterización y plasmación en la Convención Internacional sobre los Derechos de las Personas con Discapacidad. Madrid: Cinca.

Parra, B., Oriol, A. y Martínez, M. (2011): Trabajo social y enfermero en un equipo de atención precoz a la psicosis. Revista de Psicopatología y salud mental del niño y del adolescente, 18, 89-98.

PARAZElli, M. \& Bourbonnais, M. (2017). L'empowerment en travail social. Perspectives, enseignements et limites. Sciences et Actions Sociales, 6, 1-29.

SaleEbey, D. (2000). Power in the people: Strengths and hope. Advances in Social Work, 1(2), 127-136.

SEBASTIN, K.V. (2014). Tackling contemporary challenges throughs trengths based approach in Social Work. International Journal of Social Work and Human Services Practice, 2(6), 291-295. DOI: 10.13189/ijrh.2014.020613

SEN, A. (1988). ¿Igualdad de qué? en Sterling M. McMurrin (edit). Libertad, igualdad y derecho. Barcelona: Ariel.

SCHÖN, U.K., DENHOV, A. \& TOPOR, A. (2009). Social relationships as a decisive factor in recovering from severe mental illness. International Journal of Social Psychiatry, 55(4), 336-347. https://doi.org/10.1177/0020764008093686

STARnino, V.R. (2009). An Integral Approach to Mental Health Recovery: Implications for Social Work. Journal of Human Behavior in the Social Environment, 19, 820-842. https://doi.org/10.1080/10911350902988019

Tew, J., Shula, R., Slade, M., Bird, V., Melton, J \& Le Boutillier, Cl. (2012). Social Factors and Recovery from Mental Health Difficulties: A Review of 
the Evidence. British Journal of Social Work, 42 (3), 443-460. https://doi. org/10.1093/bjsw/bcr076

Toboso, M. y ARnau, M.S. (2008). La discapacidad dentro del enfoque de capacidad y funcionamiento de Amartya Sen. Araucaria Revista Iberoamericana de Filosofía, Política y Humanidades, 20, 64-94. <http://www.redalyc.org/articulo. oa? id=28212043004 > ISSN 1575-6823.

Victoria, J. A. (2013). El modelo social de la discapacidad: una cuestión de derechos humanos. Boletín Mexicano de Derecho Comparado, 46 (138), 1093-1109. https://doi.org/10.1016/S0041-8633(13)71162-1

Velarde, V. (2012). Los modelos de la discapacidad: un recorrido histórico. Revista empresa y humanidades, 1, 115-136.

Williams, CH., Almeida, M. \& Knyahnytska, Y. (2015). Towards a Biopsychosociopolitical Frame for Recovery in the Context of Mental Illness. British Journal of Social Work, 45 (1), i9-i26. https://doi.org/10.1093/bjsw/ bcv100 
Cita bibliográfica: Daniel, F; Ferreira, J. \& Monteiro, R. (2017). Propriedades Psicométricas da Geriatric Social Work Competencies Scale II [Psychometric Properties of Geriatric Social Work Competencies Scale II]. Alternativas. Cuadernos de Trabajo Social, 24, 55-66. https://doi.org/10.14198/ALTERN2017.24.04

\title{
PROPRIEDADES PSICOMÉTRICAS DA GERIATRIC SOCIAL WORK COMPETENCIES SCALE II \\ PSYCHOMETRIC PROPERTIES OF GERIATRIC SOCIAL WORK COMPETENCIES SCALE II
}

\author{
FERNANDA DANIEL* \\ fernanda-daniel@ismt.pt \\ JORGE FERREIRA** iD \\ jorge.manuel.ferreira@iscte.pt \\ ROSA MONTEIRO*** \\ monteiro.rosal4@gmail.com
}

\section{Resumo}

Introdução: A Geriatric Social Work Competencies Scale II (GSWCS II) é considerada uma referência na avaliação de competências de Assistentes Sociais Gero. O estudo teve como objetivo examinar as propriedades psicométricas da GSWCS II numa amostra de assistentes sociais portuguesas que trabalham com pessoas idosas.

Materiais e Métodos: A GSWCS II foi administrada conjuntamente com um pequeno questionário socioprofissional elaborado por nós. Foram inquiridas/os 543 assistentes sociais com média de idades de 33.05 (7.57 $=D P$ ), do sexo feminino (96.1\%) e em funções de Chefia (72.5\%).

Resultados: A consistência interna foi elevada em todas as dimensões $(\alpha>9)$. A escala apresenta correlações elevadas com todas as suas dimensões (> .85). A Análise Fatorial Confirmatória foi utilizada para testar o modelo, baseado em uma estrutura de cinco dimensões, o teste do Qui-quadrado de ajustamento $\left(\chi^{2} / g l\right)=3.129$ apresentou adequado índice de ajustamento.

Conclusão: A GSWCS II apresenta indicadores de ser uma escala válida e fidedigna.

Palavras-chave: Serviço Social, Pessoas Idosas, Competências

\begin{abstract}
Introduction: The Geriatric Social Work Competencies Scale II (GSWCS II) is considered a reference in the assessment of the competencies of Gero Social Workers. The aim of the study is to examine the psychometric properties of GSWCS II in a sample of Portuguese social workers working with elderly people.

Material and Methods: The GSWCS II was administered in conjunction with a specific designed socioprofessional questionnaire. A total of 543 social workers were interviewed, with a mean age of $33.05(7.57=S D)$, female (96.1\%) and in management functions (72.5\%).

Results: Internal consistency was verified with high values in all dimensions $(\alpha>$.9). The scale has high correlations with all its dimensions (>.85). The Confirmatory Factor Analysis was used to test the model, based on a five-dimensional structure, the chi-square test of adjustment $\left(\chi^{2} / d f\right)=3.129$ presented an acceptable index of adjustment.

Conclusion: The GSWCS II proved to be a valid and reliable scale for this population.
\end{abstract}

Key words: Social work, Elderly People, Competencies

\footnotetext{
* Doutorada em Psicologia (Desenvolvimento e Intervenção Psicológica), Doutoranda em Serviço Social, professora do Instituto Superior Miguel Torga, investigadora do Centro de Estudos e Investigação em Saúde da Universidade de Coimbra - Coimbra, Portugal. Largo da Cruz de Celas, 1. - 3000-132 Coimbra - Portugal. Tel:(+351) 239488030 - Fax:(+351) 23948803 ;

* * Doutorado em Serviço Social, professor e diretor da Licenciatura e do Doutoramento em Serviço Social do ISCTE - Instituto Universitário de Lisboa, investigador do Centro de Investigação e Estudos de Sociologia do Instituto Universitário de Lisboa - Lisboa, Portugal.

*** Doutorada em Sociologia do Estado, Direito e Administração, professora do Instituto Superior Miguel Torga, investigadora do Centro de Estudos Sociais da Universidade de Coimbra - Coimbra, Portugal.
} 


\section{Extended abstract}

Introduction: Previous studies report the existence of several factors contributing to the development of gerontological competencies in Social Work (Damron-Rodriguez, Volland, Wright, \& Hooyman, 2009; Rosen, Zlotnik, Curl, \& Green, 2000 Scharlach, Damron-Rodriguez, Robinson, \& Feldman, 2000). According to Rosen et al. (2000), the increase of the elderly population and the diversity of ageing trajectories justify the inclusion of gerontological contents within undergraduate and graduate degrees in Social Work curricula. A literature review also reveals that social workers with the knowledge and skills required to meet the needs of the elderly population are lacking. In the near future this need will be even more pressing, and therefore, the introduction of curricular contents in this area is highly recommended (Peterson, 1990). According to Damron-Rodriguez, Volland, Wright and Hooyman (2009), gerontological competences (Gero) are fundamental to ensure that all social workers are prepared to work with older adults, given that this is the population with whom they interact most. Consequently, advanced levels of gerontological skills are required for specialized geriatric practice and professional leadership in this expanding field (Damron-Rodriguez et al., 2009).

The Geriatric Social Work Competencies Scale Il (GSWCS-II) is considered a reference for the assessment of Social Workers Gero competencies. It encompasses «a listing of skills recognized by gerontological social workers as important to social workers effectively working with and on behalf of older adults and their families» (CSWE, 2008, p. 29). The current paper addresses the factor structure and psychometric properties of the GSWCS-II in a sample of Portuguese social workers working with elderly people.

\section{Material and methods}

Participants: A total of 543 social workers were interviewed, with ages between 22 and 63 years old $(M=33.05, S D=7,57)$, female $(96.1 \%)$ and in management functions (72.5\%). Most of the participants did their training in private education establishments $(\mathrm{n}=387 ; 72.5 \%)$, have finished their training in $2007(\mathrm{n}=53 ; 9.9 \%)$, are considered «Senior Social Worker» (n $=114 ; 21.3 \%)$, have leadership positions $(\mathrm{n}=332 ; 85.8 \%)$ and work mainly in Social Solidarity Associations ( $\mathrm{n}=234 ; 43.8 \%$ ).

Instruments: For data collection the GSWCS-II and a socio-professional questionnaire were used. The GSWCS-Il consists of 50 items that are subdivided into different domains: (i) Values, Ethics, and Theoretical Perspectives; (ii) Assessment; (iii) Intervention; (iv) Ageing Services, Programs, and Policies; (v) Leadership in the Practice Environment of Ageing. Each item is answered on a five-point Likert scale, where $0=$ «Not skilled at all» (I have no experience with this skill), $1=$ «Beginning skill» (I have to consciously work at this skill), $2=$ "Moderate skill» (This skill is becoming more integrated in my practice), 3 = «Advanced skill» (This skill is done with confidence and is an integral part of my practice), and $4=$ «Expert skill» (I complete this skill with sufficient mastery to teach others). The study comprised six phases: (1) Translation; (2) Back translation, (3) Similarities' analyses, (4) Back translation of dissimilar items, (5) Pilot test, and (6) Reliability and validity study.

Procedures: Data collection began with the analysis of the Portuguese Social Charter to identify the email addresses of institutions providing social responses to the elderly population in Portugal. Three thousand six hundred and fifteen institutions were identified and contacted via email. The study aims and a link to an online platform (Google Docs) for data collection were provided.

Statistical analysis: For statistical analyses SPSS (Statistical Package for Social Sciences, version 24) and AMOS software
(Analysis of Moment Structures, version 18.0) were used. Items' internal consistency (Cronbach's alpha) analysis were Pearson correlation coefficients were calculated to examine the association between the total scale and its dimensions. A Confirmatory Factor Analysis (CFA) was conducted in order to evaluate the fit of the hypothesized five-factor model to the data. The overall model adjustment quality was examined based on the following fit indexes: the normed Chi-square $(\chi 2)$ $\mathrm{gl})$, the Comparative Fit Index (CFI) and the Root Mean Square Error of Approximation (RMSEA). Quality of local adjustment was assessed by the factorial weights.

Results: Both the total GSWCS-II and its dimensions showed a high internal consistency (Cronbach's $\alpha$ >.902). Correlations between the GSWCS-II and its dimensions were also high $(r>$.815). The lowest factor weights ranged from.583 (item 1 of the dimension "Values, Ethics, and Theoretical Perspectives») to.862 (item of the dimension 7 «Aging Services, Programs, and Policies» and item 3 of the dimension «Leadership in the Practice Environment of Aging», with equal scores)

The five-factor model showed the following fit indexes: $\chi 2 /$ $\mathrm{gl}=3.129, \mathrm{CFI}=.97, \mathrm{GFI}=.890, \mathrm{TLI}=.883$, and RMSEA $=.064$.

Discussion and Conclusion: The new challenges of an ageing population and the consequent opening of new fields for social workers' professional action invites us to reflect on these technicians skills in this specific area. The need for competencies is highlighted in the scientific literature. For example, in the study of Naito-Chan, Damron-Rodriguez and Simmons (2004), conducted with four focus groups of older adults, caregivers (consumers), employers in the field of aging and recent Social Work graduates, several social workers' competencies have been identified, namely resources identification, ability to perform geriatric assessments, case management, and self-awareness skills. However, many "consumers» did not understand social workers' role. The focus on these technicians' training skills is considered a path. Tuncay and Duyan also mention the importance of skills, arguing that «staff competence (i.e., knowledge and skills) in delivering age-appropriate services are considered a primary indicator of organizational quality and a key to obtaining accreditation» (2015, p. 66). The current study examined the GSWSC-II factor structure and psychometric properties in a sample of social workers working with elderly people in Portugal. This is an instrument of interest for social workers' academic and professional community given that it maps the competencies recognized as important to all social workers working with the elderly. The GSWSC-II presented an excellent reliability, comparable to the one found by Tuncay and Duyan (2015). Correlation coefficients between GSWSC-II dimensions and the total scale were equally high. Adjustment indexes showed a reasonable to good fit, similar the one reported by Tuncay and Duyan (2015). To sum, the GSWSC-II can be seen as a reliable measure for competence self-assessment regarding basic and specialized social workers skills in the domain of working with the elderly.

The current study, besides making known to the existence of the GSWCS-II, which examines their psychometric properties from the results obtained in a sample of social workers working with elderly people in Portugal. The Geriatric Social Work Competencies Scale II presented an excellent reliability coefficient, measured by Cronbach's alpha, comparable to the one found by Tuncay and Duyan (2015). Correlation coefficients between the scale dimensions were equally high. The factor weights were higher than 6 tenths in all items. Adjustment indexes were similar the ones presented by Tuncay and Duyan (2015). To sum up, this instrument presents adequate psychometric qualities and it can and should be used to determine self-assessed competence in the basic and specialized behaviours of social workers. 


\section{Introdução}

Segundo as Nações Unidas, em 2015, 901 milhões de pessoas tinham 60 anos ou mais ( $12 \%$ da população global), apresentando este universo uma taxa de crescimento de 3,26 ao ano (2015). No ranking dos continentes é a Europa que tem a maior percentagem de população com 60 anos ou mais (24\%), contudo estima-se que num futuro próximo ocorra um envelhecimento rápido em outras partes do mundo. Em 2050 é expectável que as principais regiões do mundo, exceto África, tenham quase um quarto ou mais da sua população com 60 anos ou mais. Estima-se que o número de pessoas idosas no mundo será de 2,1 biliões em 2050 e poderá ascender a 3,2 biliões em 2100 (United Nations, Departmente of Economic and Social Affairs, 2015).

$\mathrm{Na}$ população com mais de 60 anos o subconjunto que mais tem aumentado é o dos idosos mais velhos, com 80 ou mais anos de idade. Estes deverão aumentar significativamente, de 125 milhões em 2015 para 434 milhões em 2050 e 944 milhões em 2100, sendo que 28\% viviam na Europa em 2015, devendo essa percentagem decrescer para 16\% em 2050 e para 9\% em 2100. Em contrapartida as populações de outras áreas do mundo continuarão, proporcionalmente, a aumentar de tamanho e elas próprias a envelhecer (United Nations, Departmente of Economic and Social Affairs, 2015).

Neste novo cenário de transformações apuradas pelos demógrafos verificaram-se também importantes alterações na composição dos agregados familiares, que se repercutem nas relações familiares entre as várias gerações e, consequentemente, nas gerações mais velhas. Os agregados familiares, caracterizados, há bem pouco tempo, por uma convivência intergeracional, dão progressivamente lugar a «novos arranjos familiares, ainda que o casal com filhos permaneça como o padrão de organização dominante, registra-se ligeira queda de sua freqüência, paralela a um aumento relativo das famílias unipessoais e das famílias monoparentais» (Carvalho \& Almeida, 2003, p. 110); correlacionado com estas mudanças verifica-se uma cada vez maior mobilidade dos itinerários geográficos e socioprofissionais, uma crescente participação das mulheres no mercado de trabalho, novos ritmos de vida que têm impactos no enquadramento familiar e social de pessoas idosas.

Em consequência destas transformações vários profissionais, entre os quais os/as assistentes sociais, são convocados para responder às necessidades desta população envelhecida. Segundo Scharlach, Damron-Rodriguez, Robinson e Feldman (2000) estes técnicos podem trazer contribuições únicas tanto para as pessoas idosas como para as suas famílias. No mesmo sentido Tuncay e Duyan, na senda de Scharlach, Damron-Rodriguez, Robinson e Feldman (2000), afirmam que «a necessidade de assistentes sociais com conhecimento 
gerontológico baseia-se na convicção de que os valores da profissão, as perspectivas teóricas e as competências são essenciais para melhorar o bem-estar dos idosos e suas famílias (Tuncay \& Duyan, 2015, p. 61).

A literatura reporta que são vários os fatores que contribuem para o desenvolvimento de competências gerontológicas no Serviço Social (Peterson, 1990; Rosen, Zlotnik, Curl, \& Green, 2000; Scharlach et al., 2000). Segundo Rosen et al. (Rosen et al., 2000), o aumento da população idosa e a diversidade do envelhecimento justifica a inserção de conteúdos gerontológicos nos currículos de Serviço Social. Elencam, igualmente, a carência de assistentes sociais com conhecimento e competências requeridas para satisfazer as necessidades da população idosa. Recordemos uma pesquisa realizada nos Estados Unidos, com membros da Associação Nacional de Assistentes Sociais (NASW) que demonstrou, por extrapolação, que 26\% (29.650) dos/as assistentes sociais trabalhavam normalmente com população idosa e que dos/as assistentes sociais que não trabalhavam, 62\%, referem que o conhecimento gerontológico é necessário nos seus postos de trabalho (Peterson, 1990). Reconhece-se que no futuro próximo esta necessidade será ainda mais premente, e por esse facto, sugere-se a introdução de conteúdos curriculares nesta área (Peterson, 1990). As competências gerontológicas (Gero) são assim, segundo Damron-Rodriguez, Volland, Wright e Hooyman (2009), fundamentais para garantir que todos os/as assistentes sociais estejam preparados para trabalhar com adultos mais velhos, uma vez que a maioria interage de alguma forma com essa população. Da mesma forma, são necessários níveis avançados de competências gerontológicas para a prática especializada geriátrica e liderança profissional neste campo em expansão (Damron-Rodriguez et al., 2009).

Em 2004, nos Estados Unidos da América, o Council on Social Work Education (CSWE), a Commission for Curriculum and Educational Innovation (COCEI) e a Commission for Accreditation (COA) iniciaram o processo de análise e revisão da Educational Policy and Accreditation Standards (EPAS). Após um extenso trabalho que incluiu revisão da literatura e pareceres de especialistas, identificaram-se vários pilares que orientariam este processo. A necessidade de reformular os Educational Policy and Accreditation Standards com base nos resultados dos/as alunos/as, nomeadamente nos comportamentos da prática, ou seja, o que um aluno/a deve aprender e ser capaz de fazer, levou a uma abordagem de educação baseada em competências (CSWE, 2008). É com base nesta filosofia que surgiu a Geriatric Social Work Competencies Scale II que é considerada como um conjunto de «habilidades reconhecidas pelos assistentes sociais gerontológicos como importantes para os assistentes sociais que trabalham efetivamente com e em nome de adultos mais velhos e suas 
famílias» (CSWE, 2008, p. 28). A referida escala, que contou com o apoio no seu desenvolvimento tanto do Council on Social Work Education, CSWE - Gero-Ed Center Competency Goals como do Hartford Partnership Program in Aging Education, HPPAE, começou com uma revisão abrangente da literatura de Serviço Social Gerontológico, produzindo um conjunto de 128 possíveis competências profissionais. Cópia dos 128 itens foi enviada a consultores académicos especializados em gerontologia, investigadores e profissionais. Este painel de especialistas foi convidado a avaliar os itens e sugerir supressões, adições e modificações. Um pré-teste foi, em seguida, enviado aos/às assistentes sociais. Com base nas recomendações do painel e do pré-teste, foram identificados 65 itens relativos a três grandes domínios profissionais: (i) Conhecimento sobre as pessoas idosas e suas famílias (17 itens); (ii) Habilidade profissional (32 itens); (iii) Prática profissional (16 itens). Após a revisão dos itens relativamente à redundância, clareza e especificidade para a prática gerontológica, foram pré-testados e avaliados numa amostra de conveniência. O questionário final foi distribuído a 2.400 assistentes sociais, professores e pesquisadores. A escala teve desenvolvimentos com eliminação de itens e acréscimo de uma nova subescala. Dos estudos que dão conta de algumas propriedades psicométricas o que se aproxima do estudo que pretendemos fazer foi o realizado por Tuncay e Duyan (2015) com um grupo de estudantes do bacharelato em Serviço Social na Turquia.

Neste artigo apresentaremos as características psicométricas da Geriatric Social Work Competencies Scale II administrada numa amostra de assistentes sociais portugueses que trabalham com pessoas idosas.

\section{Material e Métodos}

\section{Instrumentos}

Utilizámos nesta pesquisa além de um questionário de caracterização socioprofissional, elaborado por nós, a Geriatric Social Work Competencies Scale II.

A Escala Geriatric Social Work Competencies II é constituída por 50 itens que se subdividem em diferentes domínios: (i) Ética e perspetivas teóricas, (ii) Avaliação, (iii) Intervenção, (iv) Serviços, programas e políticas de envelhecimento, (v) Liderança no contexto de trabalho com pessoas idosas. Cada item é respondido numa escala de Likert de cinco pontos, em que 0 pontos significava «Nada competente (não tenho experiência com esta competência) », 1 ponto «Competência inicial (tenho de trabalhar para desenvolver esta competência)», 2 pontos «Competência moderada (esta competência começou a ser integrada na minha prática)», 3 pontos «Competência avançada (esta 
competência é realizada com confiança e faz parte integral da minha prática)» e 4 pontos «Competência especializada (realizo esta competência com mestria suficiente para a ensinar a outros)».

\section{Procedimentos}

Após permissão de utilização da escala, iniciou-se o processo de tradução e adaptação do instrumento na versão portuguesa, pois importava iniciar o processo que permitiria a utilização da escala por nativos língua portuguesa. O processo cumpriu seis fases: (1) Tradução do instrumento; (2) Retroversão, (3) Avaliação das dissemelhanças, (4) Retroversão de itens dissemelhantes, (5) Teste-piloto e (6) Estudo da fidedignidade (consistência interna) e validade.

No que concerne ao estudo empírico, que permitiu recolher os dados aqui apresentados, este iniciou-se com a obtenção, a partir da Carta Social, dos endereços eletrónicos das instituições em Portugal continental, com respostas sociais vocacionadas para a idade avançada (Centro de Convívio, Centro de Dia, Centro de Noite, Estrutura Residencial para Idosos e Serviço de Apoio Domiciliário). Foram identificadas e contactadas, via email, 3.615 instituições. No corpo da mensagem, além da descrição do estudo, foi igualmente enviado o link que remetia para um formulário on-line, disponibilizado na ferramenta eletrónica Google Docs.

$\mathrm{Na}$ análise e tratamento estatístico utilizamos o SPSS (Statistical Package for Social Sciences, versão 24) e o seu software opcional AMOS (Analysis of MOment Structures, versão 18.0). Numa primeira fase, realizámos a análise dos itens através da consistência interna (alpha de Cronbach), foi utilizado o coeficiente de correlação de Pearson para verificar a correlação entre a escala e as suas dimensões. Foi igualmente efetuada uma Análise Confirmatória para verificar se o modelo testado, baseado na estrutura de cinco dimensões, apresentava bons índices de ajustamento. A qualidade de ajustamento global do modelo fatorial foi avaliada de acordo com os seguintes índices: o teste do Qui-quadrado de ajustamento $\left(\chi^{2} / g l\right)$, o Comparative Fit Index (CFI) e o Root Mean Square Error of Approximation (RMSEA, P[rmsea $\leq 0.05]$ ). A qualidade do ajustamento local foi avaliada pelos pesos fatoriais.

\section{Participantes}

Os participantes eram maioritariamente do sexo feminino $(n=513 ; 96.1 \%)$ com idades compreendidas entre os 22 e os 63 anos, situando-se a idade média nos $35.05 \pm 7.56$. A maioria dos participantes fez a sua formação em estabelecimentos de ensino privado $(n=387 ; 72.5 \%)$, terminara a sua formação em 2007 
( $n=53 ; 9.9 \%)$, era «Assistente Social Principal» $(n=114 ; 21.3 \%), 332$ (85.8\%) assumia cargos de chefia e trabalhava maioritariamente em Associações de Solidariedade Social $(n=234 ; 43.8 \%)$.

Tabela 1

Caraterização Sociodemográfica e Profissional da Amostra $(n=534)$

\begin{tabular}{|c|c|c|}
\hline & $n$ & $\%$ \\
\hline \multicolumn{3}{|l|}{ Sexo } \\
\hline Masculino & 21 & 3.9 \\
\hline \multirow[t]{2}{*}{ Feminino } & 513 & 96.1 \\
\hline & $M(D P)$ & Min-Máx \\
\hline Idade & $35.05(7.57)$ & $22-63$ \\
\hline Anos de trabalho na área & $9.02(6.20)$ & $0-38$ \\
\hline Categoria & $n$ & $\%$ \\
\hline Assistente Social de $1 .^{\mathrm{a}}$ & 90 & 16.9 \\
\hline Assistente Social de $2 .^{a}$ & 79 & 14.8 \\
\hline Assistente Social de $3 .^{a}$ & 94 & 17.6 \\
\hline Assistente Social Principal & 114 & 21.3 \\
\hline Outra & 157 & 29.4 \\
\hline Funções & $n$ & $\%$ \\
\hline $\begin{array}{l}\text { Chefia (Diretor/a Técnico/a; Coordenador/a, Diretor/a } \\
\text { de Serviços, Diretor/a de Serviços/Diretor/a Técnico/a) }\end{array}$ & 387 & 72.5 \\
\hline Sem funções de Chefia & 147 & 27.5 \\
\hline Natureza Jurídica & $n$ & $\%$ \\
\hline Associação de Solidariedade Social & 234 & 43.8 \\
\hline Centro (Social) Paroquial & 102 & 19.1 \\
\hline Irmandade da Misericórdia / S.ta Casa da Misericórdia & 74 & 13.9 \\
\hline Entidade Lucrativa & 43 & 8.1 \\
\hline Fundação de Solidariedade Social & 28 & 5.2 \\
\hline Instituto de Organização Religiosa & 5 & 9 \\
\hline Associação Mutualista & 3 & .6 \\
\hline Outra & 45 & 8.4 \\
\hline
\end{tabular}

\section{Resultados}

O cálculo do alfa de Cronbach revelou que tanto a Geriatric Social Work Competencies Scale II como as suas dimensões apresentaram uma consistência 
interna elevada ( $\alpha$ de Cronbach $>$.902) respeitando os critérios descritos na literatura (Daniel, Gomes, \& Ferreira, 2015; Peterson, 1994).

Tabela 2

Consistência Interna da Escala

\begin{tabular}{|c|c|c|}
\hline & $\begin{array}{l}\text { Alfa de } \\
\text { Cronbach }\end{array}$ & $\begin{array}{l}\text { Número de } \\
\text { itens }\end{array}$ \\
\hline GSWS II & .979 & 50 \\
\hline I. Valores, Ética e Perspetivas Teóricas & .902 & 10 \\
\hline II. Avaliação & .902 & 10 \\
\hline III. Intervenção & .924 & 10 \\
\hline $\begin{array}{l}\text { IV. Serviços, programas e políticas de } \\
\text { envelhecimento }\end{array}$ & .946 & 10 \\
\hline $\begin{array}{l}\text { V. Liderança no contexto de trabalho com pessoas } \\
\text { idosas }\end{array}$ & .959 & 10 \\
\hline
\end{tabular}

As correlações entre a Geriatric Social Work Competencies II e as suas dimensões são elevadas, estatisticamente significativas, com valores superiores a.815.

Tabela 3

Correlações entre a Escala Global e as suas dimensões $(n=534)$

\begin{tabular}{|c|c|c|c|c|c|c|}
\hline & I & II & III & IV & $\mathrm{V}$ & $\begin{array}{c}\text { GSWS } \\
\text { II }\end{array}$ \\
\hline $\begin{array}{l}\text { I. Valores, Ética e Perspetivas } \\
\text { Teóricas }\end{array}$ & - & $.724^{* *}$ & $.737^{* *}$ & $.637^{* *}$ & $.631^{* *}$ & $.815^{* *}$ \\
\hline II. Avaliação & & - & $.792^{* *}$ & $.689^{* *}$ & $.643^{* *}$ & $.856^{* *}$ \\
\hline III. Intervenção & & & - & $.819^{* *}$ & $.760^{* *}$ & $.927^{* *}$ \\
\hline $\begin{array}{l}\text { IV. Serviços, programas e } \\
\text { políticas de envelhecimento }\end{array}$ & & & & - & $.829^{* *}$ & $.915^{* *}$ \\
\hline $\begin{array}{l}\text { V. Liderança no contexto de } \\
\text { trabalho com pessoas idosas }\end{array}$ & & & & & - & $.895^{* *}$ \\
\hline GSWS II & & & & & & - \\
\hline
\end{tabular}

** A correlação é significativa no nível .01 (bilateral). 
Os pesos fatoriais mais baixos (tabela 4), variaram entre um mínimo de.583 (item 1 da dimensão Valores, Ética e perspetivas teóricas) e um máximo de.862 (item 7 «Serviços, programas e políticas de envelhecimento» e item 3 «Liderança no contexto de trabalho com pessoas idosas» com pontuações iguais).

Tabela 4

Pesos de Regressão estandardizados dos itens das dimensões da GSWS II

\begin{tabular}{cccccc}
\hline Itens & $\begin{array}{c}\text { Valores, Ética } \\
\text { e perspetivas } \\
\text { teóricas }\end{array}$ & Avaliação & Intervenção & $\begin{array}{c}\text { Serviços, } \\
\text { programas e } \\
\text { políticas de } \\
\text { envelhecimento }\end{array}$ & $\begin{array}{c}\text { Liderança no } \\
\text { contexto de } \\
\text { trabalho com } \\
\text { pessoas idosas }\end{array}$ \\
\hline Item 1 & .583 & .688 & .645 & .680 & .740 \\
Item 2 & .699 & .724 & .721 & .748 & .787 \\
Item 3 & .707 & .784 & .732 & .788 & .862 \\
Item 4 & .649 & .734 & .777 & .778 & .850 \\
Item 5 & .659 & .733 & .802 & .808 & .854 \\
Item 6 & .724 & .797 & .791 & .859 & .870 \\
Item 7 & .731 & .739 & .814 & .862 & .855 \\
Item 8 & .762 & .666 & .790 & .804 & .858 \\
Item 9 & .655 & .761 & .698 & .810 & .848 \\
Item 10 & .779 & .742 & .705 & .840 & .853 \\
\hline
\end{tabular}

O modelo, como pode ser observado na Figura 1, foi testado através de uma Análise Fatorial Confirmatória. É baseado em uma estrutura quinquidimensional (matriz de intercorrelação com 50 itens), da Geriatric Social Work Competencies Scale II.

Os índices de ajustamento para a GSWCS foram compatíveis com o modelo de cinco dimensões. De acordo com a Análise Fatorial Confirmatória, o Quiquadrado de ajustamento $\left(\chi^{2} / g l\right)=3.129$ apresentam um adequado ajuste, $\mathrm{e}$ todos os índices (RMSEA =.064, CFI =.97, GFI =.890 e TLI =.883) tiveram um ajuste aceitável para um modelo de cinco dimensões. 
Figure 1

Esquema do diagrama das cinco dimensões da Geriatric Social Work Competencies Scale II

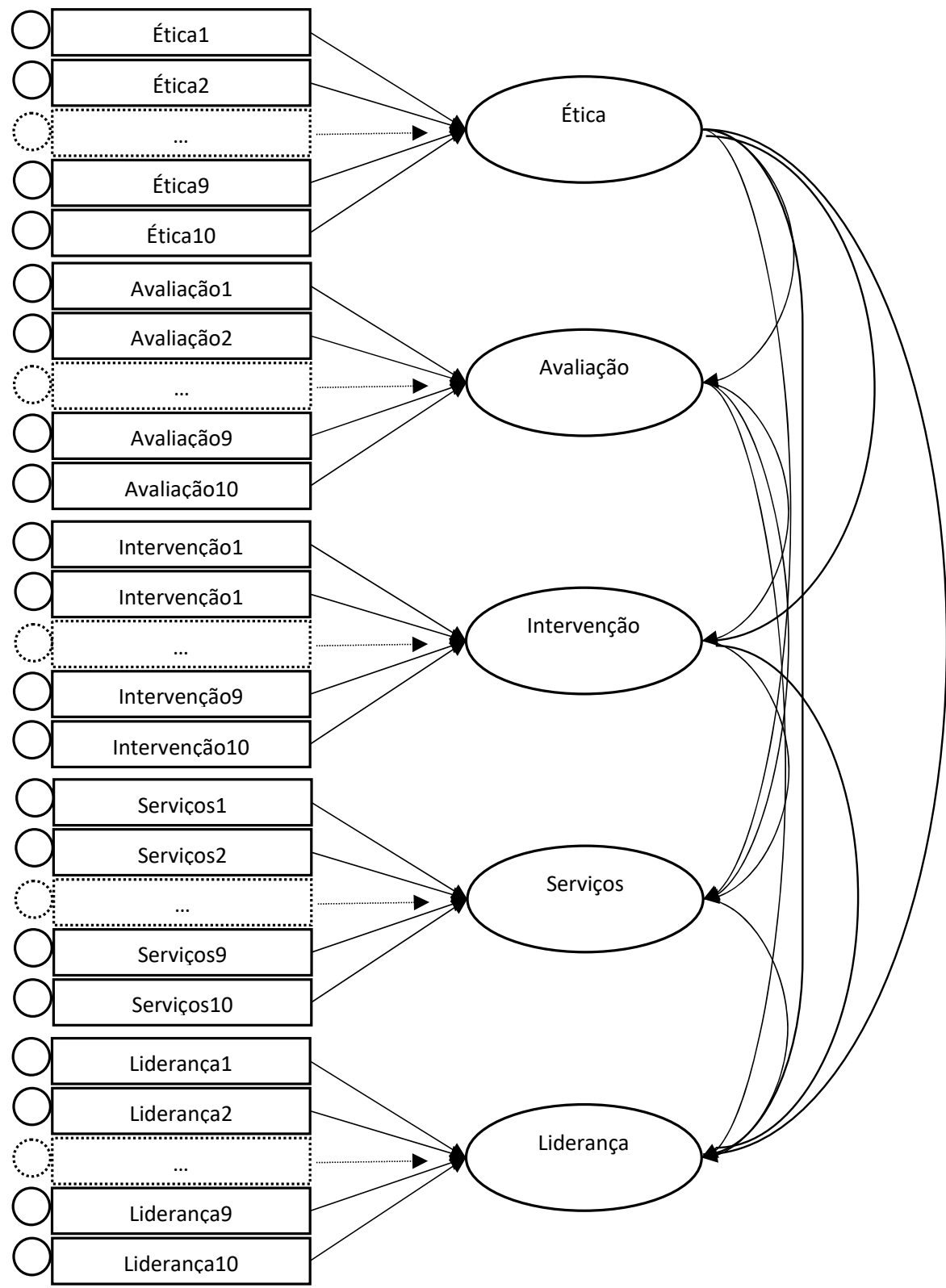

Alternativas. Cuadernos de Trabajo Social, 24, 2017, pp. 55-66 - ISSN 1133-0473 DOI: 10.14198/ALTERN2017.24.04 


\section{Discussão e Conclusão}

Os novos desafios do envelhecimento da população e a abertura consequente de novos campos de ação profissional aos/às assistentes socias convoca-nos à reflexão sobre as competências destes técnicos nesta área. A necessidade de competências estão assinaladas na literatura, o estudo de Naito-Chan, DamronRodriguez e Simmons (2004) realizado com quatro focus-group de adultos idosos, cuidadores (consumidores), empregadores no campo do envelhecimento e recém licenciados em Serviço Social é ilustrativo. Várias competências foram elencadas relativamente aos/às assistentes sociais, nomeadamente a identificação de recursos, a capacidade de realizar avaliações geriátricas, a gestão de casos, a capacidade de autoconsciência. Contudo muitos «consumidores» não entenderam o papel dos/as assistentes sociais. O enfoque das competências na formação destes técnicos é considerado um caminho. A importância das competências é também reportada por Tuncay e Duyan (2015, p. 66) apoiados em Naito-Chan, Damron-Rodriguez e Simmons (2004) «a competência do pessoal (isto é, conhecimento e habilidades) na prestação de serviços adequados à idade é considerada um indicador primário da qualidade organizacional e uma chave para a obtenção de credenciamento».

Este estudo além de dar a conhecer à comunidade académica e profissional a existência da Geriatric Social Work Competencies Scale II que mapeia as competências reconhecidas como importantes para todos/as os/as assistentes sociais que trabalham com pessoas idosas, examina as suas propriedades psicométricas a partir dos resultados obtidos em uma amostra de assistentes sociais que trabalham com pessoas idosas em Portugal. A Geriatric Social Work Competencies Scale II apresenta coeficientes de fidedignidade - consistência interna -, medidos pelo alfa de Cronbach, considerados elevados, tal como no estudo de Tuncay e Duyan (2015). Os coeficientes de correlação entre as dimensões e a escala foram igualmente elevados. Os pesos factoriais foram superiores a 6 décimas em todos os itens. Os índices de ajustamento foram entre bons e razoáveis indo ao encontro dos apresentados por Tuncay e Duyan (2015) com a escala com quarto dimensões.

Analisando os resultados obtidos, podemos afirmar que este instrumento apresenta adequadas qualidades psicométricas e desta forma pode e deve ser utilizado para determinar a competência auto-avaliada nos comportamentos básicos e especializados dos/as assistentes sociais. 


\section{Referências bibliográficas}

Carvalho, I. M. M. DE \& Almeida, P. H. DE (2003). Família e proteção social. São Paulo Em Perspectiva, 17(2), 109-122. https://doi.org/10.1590/ S0102-88392003000200012

CSWE. (2008). Advanced Gero Social Work Practice. CSWE Gero-Ed Center / Council on Social Work Education (CSWE). Recuperado de https://www. cswe.org/getattachment/Centers-Initiatives/Centers/Gero-Ed-Center/ Educational-Resources/Gero-Competencies/Practice-Guides/Practice-Guide/ GeroBrochureFINALPDF.pdf.aspx

Damron-Rodriguez, J., Volland, P. J., Wright, M. E. \& HoOyman, N. R. (2009). Competency-Based Education: Implications of the Hartford Geriatric Social Work Approach. In Hooyman (Ed.), Transforming Social Work Education: The First Decade of the Hartford Geriatric Social Work (pp. 21-50). Arlington, Virginia: Council on Social Work Education Press.

Daniel, F., Gomes, A. \& Ferreira, P. L. (2015). Contributo para a Discussão da Avaliação da Fiabilidade de um Instrumento de Medição. Revista de Enfermagem Referência, 4(7), 129-137. https://doi.org/10.12707/RIV15003

Naito-Chan, E., Damron-Rodriguez, J. \& Simmons, W. J. (2004). Identifying Competencies for Geriatric Social Work Practice. Journal of Gerontological Social Work, 43(4), 59-78. https://doi.org/10.1300/J083v43n04_05

Peterson, D. (1990). Personnel to Serve the Aging in the Field of Social Work: Implications for Educating Professionals. Social Work, (35 (5), 412-415

PETERSON, R. A. (1994). A meta-analysis of Cronbach's coefficient alpha. Journal of Consumer Research, 21(2), 381-391. https://doi.org/10.1086/209405

Rosen, A., Zlotnik, J. L., Curl, A. \& GReEn, R. (2000). The CSWE/SAGE-SW National Aging Competencies Survey Report. Council on Social Work Education. Alexandria, VA: CSWE Gero-Ed Center.

Scharlach, A., Damron-Rodriguez, J., Robinson, B. \& Feldman, R. (2000). Educating social workers for an aging society: A vision for the 21st century. Journal of Social Work Education, 36(3), 521-538.

TUNCAY, T. \& DuYAN, V. (2015). Turkish Adaptation of the Geriatric Social Work Competency Scale in a Group of Social Work Bachelor Students. Turkish Journal of Geriatrics, 18(1), 60-67.

United Nations, Department of Economic and Social AfFairs, P. D. (2015). World Population Prospects: The 2015 Revision, Key Findings and Advance Tables (241 No. ESA/P/WP). 
Cita bibliográfica: Cardona Cardona, J., Cuartero Castañer, M. E. y Campos Vidal, J. F. (2017). El diagnóstico relacional colaborativo (I) [The collaborative relational diagnosis (I)]. Alternativas. Cuadernos de Trabajo Social, 24, 67-90. https://doi.org/10.14198/ALTERN2017.24.05

\title{
EL DIAGNÓSTICO RELACIONAL COLABORATIVO (I) THE COLLABORATIVE RELATIONAL DIAGNOSIS (I)
}

\author{
JOSEFA CARDONA-CARDONA \\ Departamento de Filosofía y Trabajo Social, Universidad de las Islas Baleares, España \\ jp.cardona@uib.es \\ María Elena Cuartero-Castañer iD \\ Departamento de Filosofía y Trabajo Social, Universidad de las Islas Baleares, España \\ me.cuartero@uib.es \\ José Francisco CAMPOS-VIDAL* iD \\ Departamento de Filosofía y Trabajo Social, Universidad de las Islas Baleares, España \\ quico.campos@uib.es
}

\section{Resumen}

En la práctica del Trabajo Social, el concepto tradicional de diagnóstico remite a una sencilla fórmula: un profesional con una determinada competencia vinculada al saber y al hacer, emite un juicio en el cual se ha sintetizado e interpretado la naturaleza y la magnitud de las dificultades-necesidades de una persona, familia, grupo o comunidad. Esta visión no incorpora la dimensión relacional y cooperativa del encuentro entre el profesional y la persona en el marco de una relación de ayuda.

Este artículo considera los desarrollos epistemológicos que se han planteado desde la perspectiva de la complejidad (Morin, 1981, 2009; Maturana y Varela, 2004), las tesis del Constructivismo (Piaget, 1971, 1978), el Construccionismo Social (Goolishian y Anderson,1990) y especialmente, las vanguardias de la práctica del Trabajo Social (de Shazer, 1982 1985, 2009; White y Epston, 1990; White, 1995; Selekman, 1993).

Desde la cibernética de segundo orden, la de los sistemas observantes, lo que los profesionales creemos, pensamos y valoramos (como profesionales y como personas), influye en la visión que vamos a construir de la persona/familia. Si la subjetividad se da, ¿que impide que la valoración de la situación problema pueda ser co-construirla colaborativamente entre el profesional y sus clientes?. Desde esta perspectiva, cabe hablar de diagnóstico colaborativo. El trabajo recoge los resultados de la aplicación práctica, en condiciones experimentales y en la práctica cotidiana de profesionales, de los criterios para desarrollar una co-diagnóstico. Es útil para guiar las conversaciones con los clientes y está abierto a los ajustes que cada relación necesite en su contexto.

Palabras clave: Diagnóstico; Co-Diagnóstico; Trabajo Social relacional; Prácticas narrativas; Prácticas colaborativas.

\begin{abstract}
In the practice of Social Work, the traditional concept of diagnosis refers to a simple formula: a professional with a certain level of competence linked to knowledge and doing of the clients, issues a judgment in which the nature and magnitude of the difficulties-needs of a person, family, group or community have been synthesized and interpreted. This vision does not incorporate the relational and cooperative dimension of the encounter between the professional and the person within the framework of ahelping relationship.This article considers the epistemological developments that have been raised from the perspective of complexity (Morin, 1981, 2009, Maturana and Varela, 2004), thesis of Constructivism (Piaget, 1971, 1978), Social Constructionism (Goolishian and Anderson, 1996,1990) and especially the vanguards of the Social Work practice (Shazer, 1982, 1985, 2009; White \& Epston, 1990; White, 1995;Selekman, 1993).

From the Second-order cybernetics, the observational systems that professionals believe, think and value (as professionals and clients), influences the vision we are going to build of the person / family. To speak of an objective diagnosis, free of a biased view on the part of the professional, is to speak of a fantasy the subjectivities of the actors they value come into play. If subjectivity occurs, what prevents the assessment of the problem situation, traditionally referred to as diagnosis, can be co-built collaboratively between the professional and their clients? From this perspective, it is necessary to speak, not of diagnosis, but of collaborative diagnosis, of co-diagnosis.
\end{abstract}

Keywords: Diagnosis; Co-Diagnosis; Relational Social Work; Narrative practices; Collaborative practices

\footnotetext{
* Universidad de las Islas Baleares, Departamento de Filosofía y Trabajo Social. Ctra. de Valldemossa, s/n. Ed. Beatriu de Pinós, D3, 07122 Palma de Mallorca, España. E-mail: quico.campos@uib.es
} 


\section{Extended abstract}

In the practice of Social Work, the traditional concept of diagnosis refers to a simple formula: a professional with a certain competence linked to knowledge and doing, emits a judgment in which the nature and magnitude of the difficulties a person, family, group or community have been synthesized and interpreted.

This dominant view of the concept of diagnosis often does not incorporate the relational and cooperative dimension of the encounter between the professional and the person within the framework of a helping relationship understood as a cooperative relationship. It is usual that the idea of diagnosis is related to the interest in knowing the state of a difficult situation, with the intention of doing or proposing to do something with it to modify it.

The work considers the epistemological developments that have been raised from the perspective of complexity (Morin, 1981, 2009, Maturana and Varela, 2004), the thesis of Constructivism (Piaget, 1971, 1978), Social Constructionism (Goolishian and Anderson, 1996,1990) and especially the avantgardes of the practice of Social Work (de Shazer, 1982, 1985, 2009; White and Epston, 1990, White, 1995; Selekman, 1993).

From the second-order cybernetics, the observational systems, what professionals believe, think and value (as professionals and as people), influences the vision we are going to build of the person / family. This position moves away from the supposed neutrality of the professional observer. Our vision of what is happening, is no longer necessarily the only or the correct one, is broadened by introducing the vision of the other with the same value as that of the professional, and in this sense, the vision is adjusted and co-constructed in a process of cooperative help with the person / family.

To speak of an objective diagnosis, free from a skewed view by the professional, is to speak of a fantasy. The subjectivities of the actors that value, come into play. Who values the problem situation? The objective, rational and neutral professional of the first cybernetics?, or the help system constituted by professional and client? If subjectivity occurs, what prevents the evaluation of the problem situation, traditionally referred to as diagnosis, can be co-built collaboratively between the professional and their clients? From this perspective, it is necessary to speak, not of diagnosis, but of collaborative diagnosis, of co-diagnosis.

In the proposal we present, the diagnosis has a collaborative dimension and can be co-constructed considering certain values. It is part of a process designed to build a co-diagnosis with the person / family from a relationship of help, understood as a relationship of collaboration with the person, so as to guide the direction of their problems towards change, developing more proactive coping strategies and relying on their skills and strengths.

The Criteria that guide the practice of collaboration. Placing oneself professionally in a relationship of collaborative help with the person / family implies moving the power of professional practice, that power that unconsciously is exercised in a practice centered on the "power of the expert». It means placing oneself in a relational position of «not knowing», establishing «reciprocal relationships» where the potential possibility that the helping relationship approaches a relationship of domination is weakened (Anderson, 1997, White, 2007), allowing us travel through relational and collaborative territories whose coordinates we describe, based on our own experience and the work of various authors (Bertolino and O'Hanlon, 2001, Madsen, 2007, Madigan, 2011, White, 2016). The criteria that have guided us to formulate a collaborative diagnosis are the following:

1. The person / family is seen as an expert in their life. From the beginning of the helping relationship, their abilities and aptitudes are recognized and emphasized, giving them the same weight as our experience and professional training

2. Any aid relationship should consider the influence of the cultural and interactional context on the problem situation and the identity of the people.

3. The professionals do not renounce their knowledge, they place their role in the generation of conversations and questions that help the clients to connect with their knowledge and strengths so that from this base, they can visualize and define their own change process. Madsen (2007), warns of the importance of being cautious about when and how to offer our wisdom to clients: the knowledge of the person / family should be the first to stand out, later the knowledge built in the joint meetings and finally, if it is considered opportune, the knowledge of one's professional or personal experiences would enter into the conversation.

4. Redefine the expectations of the family about our role of «expert professional» encouraging them to participate actively in the process of change and change into collaborative professional.

5. The focus on collaboration, focuses conversations on the achievements, capabilities and resources of the person / family generating competition through discovering, evoking or highlighting 
what the family does well so that later they can transfer their knowledge to the problem situation. It is about helping clients through collaborative practices; that is, encouraging the discovery of their strengths, accessing their own knowledge, to involve them in their own process of change and help them find an «address» to their problems through conversations and questions that lead to generate alternative stories about their stories of identity, those that at the beginning of the aid relationship were colonized by deficits.

6. The definition of the problem and the objectives, arises from the conversations and questions to the family, not the professional and their theories. The participation of the family is encouraged throughout the process, from the definition of objectives, planning and methods used. From appreciative or collaboration-based research, professionals and clients initiate a joint exploration process in which help professionals ask questions with the purpose of helping clients visualize and specify the process and direction of the change.

7. Collaborative research refers to the joint work built in the study meetings (study interviews). It is not a unilateral exchange of information, on the contrary, it is a joint process that highlights the shared knowledge that emerges in the course of the conversation. In the face of differences, encourage the person / family that can qualify or finish this integration work through the recapitulation.

8. Actively listen to the effective and ineffective attempts tested by the family; that is, their own theory of change and problem, validating their experiences and letting them show us what works for them and what has not helped them change.

9. In each of the meetings of the aid process, ask for feedback to the person / family about our work, allowing them to disagree with our intervention and adjusting it based on their suggestions, generating competence in people, proximity and hope in the help relationship

10. Collaboration makes it possible to establish an alliance not only with the people who are motivated, but also with those who are supposedly reluctant. Resistance to change is not observed unilaterally, but as a stumbling block in the relationship between the aid system (social worker and family). De Shazer (1988) advocates an opening position introducing cooperative styles with the style of the family, focusing our interest in investigating what must happen in order for the change to take place, rather than focusing our efforts on combating the supposed position of no change.

\section{The process of building a collaborative} co-diagnosis

In the proposal that is presented, each of the possible areas of study must be weighed against four transversal axes (obstacles, strengths, time of permanence in the situation and life cycle in which the person / family currently transits) that will facilitate us to interrelate each one of the study areas whose contents have been part of the conversations. This operation is to move away from practices related to «summation of data collection» from the «expert professional» position of the first cybernetics. It is not a question of arriving at classifications or general categories where the subjective narrative becomes blurred and the influence of the social and cultural context becomes generalized:

Axis I: Obstacles that hinder or prevent change, deficiencies and needs, vulnerability. Axis II: Capacities, competences, knowledge, strengths and values Axis III: Time of permanence in the situation. Axis IV: Family life cycle.

Study areas to guide conversations: The work alliance generated in the help relationship. Values and purposes that make up the life of the person / family. Family structure and dynamics. Exceptions or achievements isolated. Emotional needs. Personal / family reorganization in the face of crises. Interpersonal relationships, presence or absence of experiences of resilience and contribution of significant figures to your life. Attribution, meaning, experience. Influence of the community environment and the personal network. Influence of the professional network in the maintenance and / or change of the situation. Family economy. Housing as a relational space. Work, occupation, job training and skills. Degree of motivation for change and expectations of success to overcome / manage the situation. Relationship that maintains the person / family with the disease

The collaborative help relationship is built in a process of conversations in which there are no impositions of work plans and there is no room for labeling or categorization of problems. The conversations that we contemplate mean the creation of a framework of mutual discovery. The social workers are like that, co-participants in the creation of processes that generate new meanings and alternative histories where the identity of the people is visualized in their own knowledge.

Keywords: Diagnosis; Co-Diagnosis; Relational Social Work; Narrative Practices; Collaborative practices 


\section{Introducción}

En la práctica del Trabajo Social, el concepto tradicional de diagnóstico nos remite a una sencilla fórmula: un profesional con una determinada competencia vinculada al saber y al hacer, emite un juicio en el cual se ha sintetizado e interpretado la naturaleza y la magnitud de las dificultades-necesidades de una persona, familia, grupo o comunidad (Díaz y Fernández de Castro, 2013).

Esta visión dominante a cerca del concepto de diagnóstico, término al cual nos solemos referir en el mundo profesional y académico como «diagnóstico social», frecuentemente no incorpora la dimensión relacional y cooperativa del encuentro entre el profesional y la persona en el marco de una relación de ayuda. Es habitual que la idea de diagnóstico está relacionada con el interés en conocer el estado de una situación de dificultad, con la intención de hacer o proponer hacer algo con ella para modificarla. Si pensamos en la lógica de la administración de servicios y prestaciones, típica de la gestión de servicios sociales, la crónica precariedad financiera y un casi permanente déficit de programas y proyectos que permitan cubrir carencias y dificultades, obligan a afinar la distribución de recursos escasos. En estos territorios, es lógico hablar de «recoger datos», de medir y evaluar necesidades mediante inventarios $u$ otros instrumentos que agrupan variables. Son sistemas útiles para «recoger y agrupar» las valoraciones del profesional y orientar así los diagnósticos clásicos. No obstante, dejan de lado las interrelaciones y el significado singular que otorga cada persona a los acontecimientos.

Desde la perspectiva de la relación de ayuda, esa dimensión, tan frecuente y trágicamente olvidada y que, a nuestro juicio, da sentido y naturaleza a la práctica del Trabajo Social, pensar el diagnóstico en términos clásicos (Arias y Patricia, 2016) implica no considerar los desarrollos epistemológicos que se han planteado desde la perspectiva de la complejidad (Morin, 1981, 2009; Maturana y Varela, 2004), las tesis del Constructivismo (Piaget, 1971, 1978), el Construccionismo Social (Goolishian y Anderson, 1996,1990) o las vanguardias de la práctica del Trabajo Social (de Shazer, 1982, 1985, 2009; White y Epston, 1990; White,1995; Selekman, 1993).

En todos los casos, avances actuales de la epistemología hacen especial incidencia en la relación existente entre conocimiento y poder. Desde la Teoría Crítica (Habermas,1982) hasta el post-estructuralismo (Foucault, 1975; Derrida,1971;1989) se han considerado las relaciones de poder en las interacciones humanas. Desde la perspectiva del Trabajo Social, las últimas vanguardias especialmente vinculadas a las prácticas narrativas (White y Epston, 1990) y dialógicas (Madsen, 2007; Arnkil y Seikkula, 2016), ya plantean la crítica a la idea clásica de diagnóstico. Esta crítica se inicia con las consideraciones 
que se desarrollan en el análisis de las implicaciones de poder que conllevan las formulaciones de la primera y la segunda cibernética.

Desde la perspectiva de la cibernética de primer orden, la de los sistemas observados, se mantiene la idea de la existencia de una realidad objetiva que puede ser tomada, aprehendida. El papel del profesional «neutral» es identificarla y diagnosticarla para después modificarla desde procesos de ayuda unidireccionales, en los cuales se parte de un juicio de valor que será respaldado por la narrativa social dominante. La confianza acrítica en los consensos sociales acerca de lo que está bien o mal o lo que es normal o patológico, está presente en esta visión del rol profesional y sus prácticas diagnósticas. La cibernética de segundo orden, la de los sistemas observantes, plantea que no es posible conocer (hacer observaciones y mediciones) de un sistema dado, con independencia del observador. Dicho en otros términos, lo que nosotros creemos, pensamos y valoramos como profesionales y como personas, influye en la visión que vamos a construir de la persona/familia. Esta posición se aleja de la supuesta neutralidad del profesional observador y reconoce que la observación de toda situación problema está sesgada por una carga teórica ideológica, cultural, de género y biográfica (Feyerabend, 2003). Nuestra visión de lo que está pasando, que ya no es necesariamente ni la única ni la correcta, se amplía al introducir la visión del otro con el mismo valor que la del profesional. En este sentido, la visión se va ajustando y co-construyendo en un proceso de ayuda cooperativo con la persona/familia.

Siendo así, hablar de un diagnóstico objetivo y libre de una mirada sesgada por parte del profesional, es hablar de una fantasía. Valorar el resultado de un análisis de sangre no es complejo para un profesional entrenado; valorar qué aspectos interaccionan en la situación problema, qué interacción hay entre el problema y la persona/familia, qué le sucede y, cuál es el significado que ésta le otorga a su situación, es más complejo. Entran en juego las subjetividades de los actores que valoran, ¿quién valora la situación problema?, ¿el profesional objetivo, racional y neutral de la primera cibernética?, o ¿el sistema de ayuda constituido por profesional y cliente?. Si la subjetividad se da, ¿que impide que la valoración de la situación problema, denominada tradicionalmente como diagnóstico, pueda ser co-construirla colaborativamente entre el profesional y sus clientes? Maturana (2004) probablemente nos comentaría que los seres vivos y por tanto, las personas, son capaces y autónomas para mantener y desarrollar su propia auto- organización. Es la autopoiesis, la capacidad de auto-regulación del sistema de ayuda, la que gobierna la ética, la organización y la pragmática del desarrollo y de la historia compartida del mismo. Desde esta 
perspectiva, no cabe hablar de diagnóstico, sino de diagnóstico colaborativo, de co-diagnóstico.

Como profesión hemos ido cambiando el lenguaje y el discurso (Rodríguez, 2013), integrando conceptos propios de la segunda cibernética. No obstante, el cambio epistemológico aún no se ha introducido en la práctica cotidiana, en el cómo desarrollamos la práctica, entendida esta como una relación de ayuda cooperativa. Sin renunciar a nuestros orígenes, tal vez este sea un buen momento para introducir nuevas cartografías y reorientar la dirección de la práctica hacia un Trabajo Social relacional y cooperativo.

En la propuesta que presentamos, el diagnóstico tiene una dimensión relacional y colaborativa. Forma parte de un proceso destinado a construir con la persona/familia una relación de ayuda, entendiéndola como una relación significativa basada en la alianza y construida sobre un marco de colaboración.

\subsection{Notas metodológicas}

Cómo colaborar con las personas ha sido una de nuestras inquietudes en estos últimos años. La propuesta que se presenta es el resultado de una proceso sistemático de estudio de los principales autores que, desde las vanguardias del Trabajo Social (Selekman,1996, White y Epston, 1993; Lipchik, 2005; de Sheazer, 2009, Arnkill, 2016 o Madigan, 2011), han ampliado e innovado las prácticas narrativas, colaborativas y dialógicas formuladas también por Andersen (1994), Anderson (1999), Freeman (2001, 2005) o Matsen (2007) entre otros. Se han sintetizado estas aportaciones y se han operativizado para ser aplicadas en un contexto experimental controlado, en el marco de la Universidad de las Islas Baleares. Los resultados de esta experimentación se han desarrollado en contextos laborales diversos, y su aplicación ha permitido experimentar en la generación de espacios cooperativos mediante conversaciones y preguntas.

No hemos buscado establecer clasificaciones o categorías agrupables, porque entendemos que este proceder opaca la voz y las características singulares de las personas y porque la influencia de la cultura y el contexto social en la vida de las personas no queda visibilizado. Nuestra intención es compartir con las personas lectoras una reflexión crítica, además de una guía que nos ha resultado útil para ordenar y sistematizar el conocimiento co-construido a lo largo de las conversaciones colaborativas. Ello nos ha permitido construir un diagnóstico relacional y cooperativo donde las voces y los significados de las personas queden reflejados, ya que desde el inicio de la relación de ayuda, el proceso se ha tejido cooperativamente. 


\section{Bases relacionales que han guiado las conversaciones cooperativas en la relación de ayuda}

Generar un espacio conversacional cooperativo se traduce en generar un territorio donde la persona percibe que la relación de ayuda es fluida, basada en el respeto, la confianza y el afecto. Ello, nos emplaza a introducir cambios en la manera de observar, pensar e intervenir. En este sentido, Bolaños (2008), plantea que

«construir un espacio cooperativo es algo que va mucho más allá de las técnicas utilizadas para encuadrar el proceso. Este espacio no es el requisito para que los cambios ocurran, es el cambio mismo. Se trata de una nueva realidad construida conjuntamente». (p.121)

A continuación, pasamos a describir los criterios que nos han guiado para establecer un marco cooperativo en la relación de ayuda y que por tanto, sustentan que el proceso de construcción del diagnóstico en Trabajo Social, sea relacional y colaborativo. Dichos criterios, están fundamentados en el trabajo de diversos autores (Bertolino y O'Hanlon, 2001; Morgan, 2004; Madsen, 2007; Madigan, 2011, White, 2016), y en nuestra propia experiencia. Pasamos a describirlos:

2.1 Desde el primer contacto se genera una relación de respeto con las personas, otorgándoles un papel protagonista en la conducción del proceso de ayuda y situándolas como expertas de sus vidas. Se trata de redefinir las expectativas de la familia sobre nuestro papel de «profesional experto» alentándolos a que participen activamente en su proceso de cambio y resituándonos como profesionales que colaboramos junto a ellos. Desde el inicio de la relación de ayuda, se reconocen y subrayan sus capacidades y aptitudes, dándoles el mismo peso que a nuestra experiencia y formación profesional.

El Trabajador/a Social trata de generar un contexto de ayuda basado en la colaboración y la alianza de ayuda mediante prácticas destinadas a: Estimular a la persona para que se manifieste sobre sus objetivos y sus metas; explicando cómo se podría llevar a cabo la intervención y así pueda aportar su parecer y su experiencia; estimulando su participación en el diseño del proceso de ayuda; valorando y amplificando cada pequeño cambio; generando pequeñas expectativas y validando su experiencia subjetiva.

2.2 Los encuentros de estudio (entrevistas de estudio), constituyen una investigación colaborativa, es decir, un trabajo conjunto que destaca el conocimiento compartido en el curso de las conversaciones. No es un intercambio unidireccional de información. El Trabajador/a Social sabe que su punto de vista es uno 
más, no el verdadero y se sitúa conscientemente en un espacio colaborativo, de respeto e inclusión del otro, para comprender junto a este otro. El producto final es una realidad tejida, co-construida en el transitar de un encuentro a otro.

Recapitular y ajustar en cada encuentro, se convierten en pasos necesarios para llegar a este producto final. El Trabajador/a Social comparte mediante la recapitulación sus impresiones sobre lo acontecido en el encuentro y sobre el proceso transitado hasta el momento. Posteriormente, ajustar dichas impresiones solicitando a la persona si desea introducir algún aspecto que no se ha tenido en cuenta. Con esta práctica, situamos a la persona como experta que colabora de forma activa. Sus matizaciones o divergencias son importantes para nosotros y para el proceso de ayuda. En este ajustar con el otro, el Trabajador/a Social realiza un movimiento de apertura, flexibiliza su mirada e incluye la del otro, estos pasos de baile, van conformando el espacio colaborativo donde se apoyará la relación de ayuda.

2.3 Tener en cuenta la influencia de la cultural y el contexto social e interaccional en la situación problema y en la identidad de las personas.

2.4 La definición del problema y la de los objetivos, debe surgir de las conversaciones y preguntas a la familia, no del profesional y sus teorías. La participación de la familia es fomentada durante todo el proceso, desde la definición de objetivos, la planificación y los métodos utilizados. Desde la investigación apreciativa o basada en la colaboración, los profesionales y los clientes inician un proceso de exploración conjunta en la que los profesionales de ayuda plantean preguntas con propósito de ayudar a los clientes a visualizar y concretar el proceso y la dirección del cambio.

2.5 Centrar las conversaciones en los logros, las capacidades y los recursos de la persona/familia generando así competencia a través de descubrir, evocar y subrayar aquello que la familia hace bien para que posteriormente pueda transferir sus saberes a la situación problema. Desde el primer encuentro, se trata de ayudar a los clientes a través de prácticas colaborativas. Ello significa fomentar el descubrimiento de sus fortalezas, accediendo a sus propios conocimientos, para implicarlas en su propio proceso de cambio y ayudarles a encontrar una «dirección» a sus problemas. Mediante conversaciones y preguntas que enriquezcan su historia, descubriendo detalles que quedaron eclipsados por el predominio del problema.

2.6 Escuchar activamente los intentos eficaces e ineficaces ensayados por la familia, es decir, su propia teoría del cambio y del problema, validando sus experiencias 
y dejando que nos enseñen qué funciona para ellos y qué no les ha ayudado a cambiar. Ellos son los que saben de sus vidas.

2.7 En cada uno de los encuentros del proceso de ayuda, solicitar feedback a la persona/ familia sobre nuestro trabajo, dando margen a que puedan discrepar de nuestra intervención y ajustándola en base a sus sugerencias. Solicitar feedback es una práctica cooperativa y como tal, genera competencia y proximidad con la persona y esperanza en la relación de ayuda. Trasmitimos que sus opiniones son importantes para nosotros y para el proceso, y que además somos conscientes que nuestra opinión sobre el encuentro, es parcial y necesitamos de su feedback para ajustar la manera en que estamos acompañando en el proceso de ayuda.

2.8 Cuestionar la resistencia al cambio, centrando nuestro interés en investigar qué debe ocurrir para que el cambio se dé, más que centrar nuestros esfuerzos en combatir la supuesta postura de no cambio. La resistencia al cambio no será observada unilateralmente, sino como un atasco en la relación del sistema de ayuda (Trabajador/a Social y familia). Así, ubicarnos en un marco de colaboración nos va a permitir establecer una conexión, no sólo con las personas que están motivadas, sino también con aquellas que son supuestamente reacias. Somos conscientes que el nivel de co-construcción alcanzado no se dará por igual en todas las personas/familias, ni en todos los contextos profesionales. A pesar de ello, mantenemos que la relación de ayuda debe guiarse en todos los casos, aún en los más complejos o vulnerables, por prácticas cooperativas, realizando anclajes en los puntos de conexión y ampliando la curiosidad genuina hacia pequeños puntos de acuerdo. (Madsen, 2007; O'Hanlon y Bertolino, 2001; Lipckic, 2005).

2.9 Mantener un ejercicio profesional discreto. Por último, se trata de cuestionar la superioridad implícita o explicita del profesional sobre la persona que solicita ayuda. Ubicarse en una postura profesional de «no saber» no significa renunciar de los conocimientos, sino colocar el papel del Trabajador/a Social en la generación de conversaciones y preguntas que ayuden a los clientes a conectar con sus propios conocimientos y fortalezas, y desde ahí, puedan visualizar y definir su propio proceso de cambio. Madsen (2007), advierte de la importancia de ser cauteloso a acerca de cuándo y cómo ofrecer nuestra sabiduría a los clientes. Los saberes de la persona/familia deben ser los primeros en destacarse, posteriormente los conocimientos construidos en los encuentros conjuntos y por último, si se valora oportuno, entraría en la conversación el conocimiento de las propias experiencias profesionales o personales. 


\section{Diagnóstico relacional colaborativo. El proceso de construir un co- diagnóstico}

En el apartado anterior, hemos señalado las bases relacionales sobre las que nos hemos apoyado para construir conversaciones colaborativas. Ahora entraremos a describir cómo éstas configuran el proceso de diagnóstico colaborativo que proponemos. Éste, tiene sentido en un proceso de ayuda que desde los primeros encuentros, se construye desde la colaboración activa de la persona/ familia. Para nosotros no se trata simplemente de hacer un diagnóstico sistematizando la información como un producto final. Hay un paso clave, se trata de generar un espacio cooperativo en cada encuentro que permita compartir los conocimientos co-contruidos y que ellos, como expertos que son, puedan ajustar y puntualizar la información sobre sus vidas.

El sistema de ayuda estará en condiciones de co-construir un diagnóstico relacional y colaborativo, si las conversaciones (entrevistas) de estudio han sido espacios de investigación conjunta, y estas han facilitado a los clientes acceder a su propio saber (Madsen, 2007); si han permitido acercarnos a definiciones compartidas sobre el problema y si han permitido descubrir juntos los valores, las fortalezas y las competencias existentes o potenciales de las personas.

Este momento de diagnóstico, se concreta cuando consensuamos con el cliente que el conocimiento sobre la situación es suficiente para identificar la influencia de las fortalezas y del problema en su vida. Si este conocimiento es compartido por el sistema de ayuda, servirá de plataforma sobre la cual coconstruir el proyecto de intervención para el cambio.

Desde la formulación que presentamos, el proceso de construcción del co-diagnóstico comporta diferentes pasos:

1.Ordenar y sistematizar el conocimiento y los micro saberes construidos a lo largo de las conversaciones colaborativas, mantenidas en los encuentros de estudio (diagnóstico relacional colaborativo).

2.Crear una «historia compartida» en base a esta ordenación. Debe ser una historia fruto de las conversaciones colaborativas y será compartida y ajustada con la persona/ familia en un encuentro que denominamos de co-diagnóstico.

3. Encuentro de co-diagnóstico: compartir y ajustar «la historia compartida» con la persona/familia, construyendo así el co-diagnóstico, y finalmente, acordar los contextos de intervención profesional sobre los que se desarrollará la intervención para el cambio.

En este artículo, por motivos de extensión, nos centraremos en el paso 1 (Ordenar y sistematizar el conocimiento y los micro saberes construidos a lo largo de las conversaciones colaborativas, mantenidas en los encuentros de estudio 
-diagnóstico relacional colaborativo), que a su vez, se centra en unos ejes y unas áreas de estudio.

A continuación, pasamos a describir una guía para este primer paso

\subsection{Guía para ordenar y sistematizar los conocimientos co-construidos en las conversaciones colaborativas mantenidas en los encuentros de estudio (diagnóstico relacional colaborativo)}

A continuación presentamos los cuatro ejes, que de forma transversal, deberán interaccionar con las quince áreas de estudio que presentaremos en el próximo punto.

Eje I: Obstáculos que frenan o impiden el cambio, carencias y necesidades, vulnerabilidad.

¿Cuáles son los obstáculos que influyen manteniendo la situación de malestar y/o el problema?; ¿Cuáles impiden que la persona/familia pueda salir por si misma de esta situación?

Eje II: Capacidades, competencias, conocimientos, fortalezas y valores ¿Si éstos son reforzados o potenciados pueden facilitar el cambio?

En ambos ejes, se trata de balancear los contenidos de las conversaciones construidas en cada una de las áreas de estudio y valorar si influyen manteniendo el problema (trabas que impiden avanzar o mejorar) o influyen en forma de fuerzas dinámicas constructivas (recursos personales activos o potenciales) que si son reforzadas pueden facilitar el cambio.

Eje III: Tiempo de permanencia en la situación.

La variable «Tiempo» influirá directamente en el pronóstico de mejora o cambio. Por ejemplo, una situación problema estable durante diez años, probablemente habrá influido en cronificar patrones de interacción, maneras de hacer que requerirán intervenciones más complejas y a más largo plazo que si nos encontramos ante una situación circunstancial, una crisis con poco recorrido en el tiempo. Se trata de valorar la influencia del tiempo en cada una de las áreas. ¿La situación problema se ha mantenido en un periodo largo de tiempo? (cronicidad); ¿En qué áreas ha podido mantenerse la estabilidad y la situación problema no se ha instaurado?(fortalezas); ¿Se trata de una situación estable (estructural) o una situación circunstancial (crítica) en la vida de esta persona/familia?.

También hay que considerar la interacción del Eje III (el tiempo) con los otros ejes: ¿cómo ha influido el tiempo de permanencia en los otros ejes?; Eje I: ¿qué se ha instalado en su vida, que influye como obstáculo para el cambio?; 
Eje II: ¿qué fortalezas, competencias, habilidades se han podido mantener?; Eje IV:¿Cómo ha influido en su desarrollo evolutivo, la permanencia de un largo periodo de tiempo aislado?; ¿Qué retos no pudo, puede o podrá vivir?.

Eje IV: Ciclo vital familiar.

Cada etapa del ciclo vital familiar, necesita condiciones particulares para que el desarrollo evolutivo pueda ser más o menos fluido. Se trata de relacionar cada una de las áreas con las condiciones necesarias que debería disponer la familia para seguir su propio desarrollo evolutivo. ¿Existen condiciones para que los miembros de la familia puedan seguir gestionando los retos de sus etapas de desarrollo?

\section{2 Áreas de estudio para guiar las conversaciones}

Pasamos a proponer un conjunto de áreas de estudio (temas para las conversaciones). En cada una de ellas hemos introduciendo preguntas que podrían facilitar la sistematización de la información construida. Dichas preguntas, van dirigidas a despertar y potenciar el conocimiento y el saber de la persona/familia así como a generar una «investigación conjunta» sobre la influencia mutua entre la persona y el problema. Cada lector puede ampliar o reducir el tema de las conversaciones según el interés y/o la necesidad de la persona/familia.

Además, debido a la limitación de espacio, sólo en las cuatro primeras áreas de estudio hemos introducido, a modo de ejemplo, auto-preguntas que pueden ayudar a los profesionales a balancear los ejes con las áreas de estudio. Presentamos estas preguntas como preguntas de balanceo.

\subsubsection{La alianza de trabajo generada en la relación de ayuda}

Hacemos referencia al establecimiento de una alianza de ayuda con la persona/ familia como hilo conductor de una relación colaborativa. Sin ella, todo este proceso no será posible.

La relación basada en la alianza de ayuda construye un vínculo entre profesional y cliente. Ambos son importantes uno para otro, aprenden uno de otro y este hecho en sí es lo que genera la confianza del cliente en una relación constructiva (Casement,1989).

Una relación basada en la alianza terapéutica o de ayuda, conlleva hilvanar cuatro dimensiones colaborativas: a) enganche en el proceso de ayuda; b) conexión emocional con el trabajador social; c) seguridad dentro del sistema de ayuda, y d) sentido de compartir el propósito de la relación de ayuda en la familia (Friedlander, Escudero y Heatherington, 2009). Supone ofrecer un espacio seguro en donde la persona o familia pueda confiar, ofrecerse como 
una figura próxima y disponible «estar para ellos», utilizar un tono emocional en los encuentros y construir un vínculo emocional con las personas. Implica establecer un propósito común sobre los temas que nos llevan a trabajar juntos y manifestar verdadera motivación para ello. Creer y ver que el cambio es posible en esta familia. Se traduce en visualizarnos acompañando a la familia desde la cooperación, como uno más y finalmente, solicitar a la persona/ familia un feedback sobre cómo nos perciben, sobre nuestra manera de trabajar con ellos. (Escudero y López, 2003).

La construcción del contexto de intervención para el cambio (Cardona y Campos, 2009; Cardona, 2012) y la construcción de una alianza de ayuda en la dimensión del enganche definida por Friedlander, Escudero y Heatherington (2009), comparten prácticas relacionales que definen el espacio terapéutico de intervención.

A través de estas prácticas, la persona/familia puede percibir que su colaboración en los objetivos y las metas es estimulada y valorada por el Trabajador/a Social. Percibir que sus opiniones sobre cómo va a transcurrir la intervención son tenidas en cuenta, que sus puntos fuertes son potenciados. Puede experimentar que su persona es valorada de forma distinta al problema y el problema como algo diferente a su persona. Experimentar que los pequeños cambios que se van logrando en el transcurso de la relación de ayuda son valorados y amplificados. Finalmente, la familia puede percibir que el clima que se genera en la relación de ayuda transmite esperanza e ilusión en que es posible algún tipo de cambio (Friedlander, Escudero y Heatherington, 2009). Podríamos preguntarnos:

¿Cómo hemos estimulando a los clientes para que se manifiesten sobre sus objetivos y sus metas?; ¿Cómo hemos explicado detalladamente la forma en se va a llevar a cabo la intervención?; ¿De qué manera hemos dejado espacio para que puedan opinar y finalmente ajustar la intervención a sus preferencias, fruto de su saber y de experiencias anteriores?; ¿Cómo hemos estimulando su participación en diseñar el proceso de ayuda?; En cada encuentro, ¿de qué manera hemos valorado y amplificado cada pequeño cambio que se haya producido?; ¿Cómo hemos generado pequeñas expectativas y validado su experiencia subjetiva?

Preguntas de balanceo:

Eje I. ¿Qué aspectos de la relación establecida impiden el cambio?; ¿Qué trabas en la alianza de trabajo están influyendo en el no cambio?

Eje II: ¿Cómo hemos solicitado un feedback al respecto de nuestra forma de conducir la relación de ayuda en cada encuentro? Ya que ellos son los protagonistas 
del proceso, ¿hemos manifestado que su opinión es importante y necesaria para nosotros y para la relación de ayuda? Desde nuestro punto de vista, ise ha generado una alianza de ayuda en la relación? Desde el punto de vista de la personalfamilia, ¿se ha generado confianza, respeto, alianza?; La confianza, el respeto, la alianza construida ijuega como elemento favorecedor del cambio?

Eje III. ¿La relación basada en la alianza de trabajo, está ayudando a mantener estabilidad en la familia?

Eje IV: ¿La relación de ayuda ha contribuido a una mejor gestión a la hora de pasar a otra etapa vital?

\subsubsection{Valores y propósitos que conforman la vida de la persona/familia}

Se trata de conversar sobre aquello a lo la persona/familia da más importancia, qué aprecian más, qué les merece su respeto, qué atesoran y no desean perder porque es apreciado y valorado. Qué propósitos tienen para su vida, qué razones o intenciones los llevan a querer cambiar.

En el caso de que el problema esté centrado en una persona o en una relación: ¿Qué cualidades positivas la representan mejor, qué don posee, qué es lo destacable en ella?; ¿En qué situaciones aparecen más estas cualidades especiales?; ¿Cuándo lo descubrió por primera vez y qué significó este descubrimiento?; ¿De quién pudo aprender estas virtudes?; ¿Son dones y cualidades familiares?; ¿Qué otras personas comparten que la persona posee estos dones y cualidades especiales?.

Preguntas de balanceo:

Eje I. ¿qué valores detectados impiden el cambio?; ¿Cuáles están influyendo en el no cambio?

Eje II. ¿Cuáles están activos y presentes?; ¿Cuáles, si son potenciados podrían facilitar el cambio?

Eje III. ¿Algún valor o propósito está influyendo en que la situación se estabilice en el tiempo?; ¿Qué propósitos y valores han ayudado a mantener estabilidad en la familia?

Eje IV. ¿Los valores y propósitos de la familia, obstaculizan o permiten que la familia pueda transitar de una etapa a otra del ciclo vital?

\subsubsection{Estructura y dinámica familiar}

Es resultado de las conversaciones mantenidas sobre su mapa de relaciones (genograma), que identifican problemas o situaciones de malestar. Éstas también motivan a la familia a conversar sobre sus propios recursos y sus puntos fuertes, subrayando las interacciones que han reflejado fuerza y competencia 
entre ellos, o bien aquellos momentos en que la familia ha manifestado que lo pasan bien cuando comparten momentos juntos, o simplemente compartiendo satisfacción ante lo que la familia manifiesta hacer bien.

Es recomendable explorar: a) valores, rituales y costumbres que ha mantenido la persona/familia a pesar de la situación problema; b) reglas, mitos familiares y sistema de creencias; c) lealtades familiares y delegaciones, jerarquía familiar; d) límites entre los miembros y hacia el exterior; e) alianzas, coaliciones y triángulos entre los subsistemas, así como patrones de comunicación entre los miembros y tipos de cohesión familiar.

Preguntas de balanceo:

Eje I. ¿Qué límites y reglas entre subsistemas impiden el cambio?; ¿Cuáles están influyendo para no cambiar?

Eje II. ¿Qué alianzas están presentes?; ¿Qué posibles alianzas, si son potenciadas pueden facilitar el cambio?

Eje III. ¿Cuánto tiempo hace que la comunicación se mantiene bloqueada/cordial?; ¿Cómo está influyendo en que la situación se estabilice o cronifique en el tiempo?; ¿Qué rituales y costumbres han ayudado a mantener estabilidad en la familia a lo larga del tiempo, a pesar de la situación problema?

Eje IV. ¿El nivel de cohesión en la familia, facilita que los adolescentes puedan intercambiar experiencias con otros jóvenes?

\subsubsection{Excepciones o logros aislados}

De Shazer (1990), define las excepciones como aquellas veces en que el problema no ha estado presente: ¿En qué situaciones la persona/familia ha logrado esquivar el problema?; ¿Qué hicieron?; ¿En qué circunstancias ha podido combatirlo, hacerle frente y lograr que su influencia fuera menor? ¿Qué ha representado este logro, aunque temporal, para la persona/familia?; ¿Qué personas de su entorno se dieron cuenta de que, en esta ocasión, el problema había sido desbancado? y ¿Qué vieron en ellos de diferente para saber que algo había cambiado?; ¿Qué han significado estos logros aislados en su vida, algo positivo, negativo, un poco de ambos?

Según la persona/familia, ¿Qué cosas deberían ocurrir para que la influencia del problema en sus vidas fuera menor, para que estos logros aislados pasen a ser permanentes en su vida?

Preguntas de balanceo:

Eje I: No procede 
Eje II. ¿Qué logró hacer la familia para esquivar, en esta ocasión, el malestar?; ¿Qué cosas, si son potenciadas pueden influir aumentando las ocasiones donde el problema no esté?

Eje III. ¿Cuál fue la última vez en que la familia vivió un logro?; ¿Qué circunstancias o cosas son permanentes en su vida?; ¿Qué cosas logran mantener estabilidad familiar?

Eje IV. ¿Este logro aislado, facilitó alguna experiencia de apertura en el desarrollo evolutivo de la persona o de algún miembro de la familia?

\subsubsection{Necesidades emocionales}

Tenemos en consideración a Glasser (1975), que ampliando la Teoría de las Necesidades de Maslow (1954), plantea que, entre otras, las necesidades psicoemocionales de los seres humanos pueden sintetizarse en dos. Su insatisfacción generará, según Glasser, profundo malestar y una significativa precariedad personal y relacional. En primer lugar, el autor señala la necesidad de amar y de ser amado, es decir, la necesidad de vincularse, de ser confirmado como sujeto, la necesidad de pertenencia e inclusión, la necesidad de sentirse significativo para otros. En segundo lugar, la necesidad de dar sentido a nuestras vidas, es decir, darles un significado trascendente en lo concreto, de experimentar subjetiva y relacionalmente que nuestra vida tiene un sentido. En esta amplia línea, las conversaciones pueden desarrollarse en los siguientes territorios:

¿Qué papel juega el contexto socio-cultural en el mantenimiento del problema o en los recursos que faciliten el cambio?; ¿Qué mapa de relaciones ha contribuido a la construcción de su identidad actual?; ¿Cuál ha sido su historia de relaciones de apego y desapego, de dependencia o autonomía?; ¿Qué metas y propósitos tiene para su vida?, ¿Qué cree merecer?, ¿Cuál es la visión de sí mismo?; En la actualidad, ¿cuál es su capacidad para generar vínculos afectivos en los que apoyarse?; ¿Qué personas, propósitos, sueños, acontecimientos, hechos o circunstancias han facilitado que la persona haya podido seguir funcionando a pesar de su sufrimiento o de su malestar?; ¿Qué ayudas o influencias ha tenido para construir una visión de sí misma constructiva (autoestima)?; ¿Reconoce y/o pone en valor los logros que ha alcanzado? (auto-concepto)

\subsubsection{Reorganización personal/familiar ante las crisis}

Conversaciones mantenidas sobre cómo se ha reorganizado la persona/familia ante una pérdida, una crisis propia de la vida cotidiana o bien un acontecimiento crítico o traumático (Rubin y Bloch, 2001; Wlash, 2005): 
¿A qué eventos críticos se enfrenta la persona/familia que son los propios de la etapa vital que atraviesa?; ¿Cómo los gestionan, cómo se enfrentan a ellos?; ¿Cuál es su estilo habitual de resolución de conflictos o de afrontamiento de las crisis (asertivo, agresivo, introvertido o un poco de cada uno)?; ¿Qué le impide sobrellevar la situación por si misma?; Según la persona, ¿qué recursos internos y externos le ayudarían a gestionar mejor la situación?; ¿Cuáles son las necesidades propias del ciclo vital familiar que atraviesan y en qué medida están cubiertas?; ¿La situación problema que vive la familia, permite a sus miembros continuar evolucionando en su vida o por el contrario deben aplazar su desarrollo por la influencia del problema?

La persona/familia, ¿tiene disposición para pedir ayuda exterior?; ¿Puede introducir flexibilidad ante posibles necesidades que debe afrontar?; ¿Puede introducir temporalmente cambios en su vida para hacer frente a la situación?; ¿Cuál es su fuerza ante la situación crítica que atraviesa?; ¿Qué les sostiene?; ¿Cómo logran mantener una relación con la esperanza, en que las cosas mejoraran? y ¿Cuál es su vulnerabilidad?

\subsubsection{Relaciones interpersonales, presencia o ausencia de experiencias de} resiliencia y contribución de figuras significativas a su vida

Es el resultado de las conversaciones mantenidas a cerca de su mapa de relaciones (eco mapa) con figuras que han sido significativas en su vida. Hemos tenido especial cuidado en no construir preguntas en una sola dimensión. Buscamos introducir preguntas que permitan pasar de un relato unidireccional a uno bidireccional, en el sentido de que la persona, no fue una receptora pasiva de la ayuda de estas figuras significativas, sino que ella también contribuyó en sus vidas (White, 2016). Algo vieron en la persona estas figuras significativas, que la ayudaron y en este ayudar, enriquecieron también sus vidas.

Otro aspecto a tener en cuenta, es ampliar el concepto de persona significativa. Generalmente nos referimos a personas reales y vivas que forman parte de nuestra vida. También forman parte de nuestras vidas e influyen en ella, personas que ya no están pero continúan ejerciendo una influencia en nosotros, así como personajes o héroes de ficción que han inspirado valores o nos han inspirado en nuestra vida (White, 2016).

¿Qué tipo de relaciones mantiene con su red de amigos, compañeros de trabajo y vecinos?; ¿Cuál es la cantidad, la calidad y la frecuencia de dichas relaciones?; ¿Cuál es la naturaleza de las relaciones que mantiene (independiente, pasiva, sumisa, dependiente...un poco de cada una de ellas)?; ¿Qué personas son significativas en la vida de la persona/familia y que además, puedan contar con ellas en este momento crítico?; ¿Qué relaciones y apoyos 
sociales cree la persona que es necesario mantener, potenciar o reactivar en su vida?; ¿Hubo en su vida figuras significativas que contribuyeron en sus valores, aspiraciones, sueños?; ¿Qué valores le ayudaron?; ¿Cómo contribuyeron a la vida y a la formación de su identidad?; ¿Qué vieron y reconocieron estas figuras, que para su familia no era valorado ni visible?; ¿En qué aspectos contribuyó la persona a la vida de las personas significativas?; ¿Existe conciencia en la persona sobre lo que pudo significar para ellas ayudarla?; ¿Qué había en ella, como persona, que contribuyó a que estas figuras se fijaran en ella y la ayudaran?; ¿Existe la conciencia que no fue una receptora pasiva de la ayuda de estas figuras significativas, sino que ella también contribuyó en sus vidas?; En la actualidad, ¿algunos de sus valores siguen presentes y son testimonio de la experiencia compartida con estas personas?; ¿Qué valores y saberes atesorados al compartir experiencias con estas personas, quiere que sigan presentes en el futuro de su vida?.

\subsubsection{Atribución, significado, vivencia}

¿A qué atribuye la persona la situación problema que está atravesando? Se trata de construir conversaciones sobre el lugar que ocupa la atribución en su vida (locus de control interno y externo), o si por el contrario, atribuye que el problema es debido a aspectos referidos de su persona, a causas externas o un poco de ambas (Bandura, 1999):

¿Qué peso tiene dicha atribución en el proceso de cambio?; ¿Cómo traba o cómo superación?; ¿Qué significados y consecuencias tiene el problema para la persona, para su familia, para su red, para la sociedad en la que vive actualmente?; ¿Qué significados y consecuencias tiene el problema en su cultura, en su comunidad de origen?; ¿Cómo vive esta situación?; ¿Cómo un bache temporal, como un fracaso personal?; ¿Qué intentos de solución le han funcionado?; ¿Qué intentos no llegaron a buen puerto?; ¿Cuál es su actitud general ante la vida y ante esta situación en concreto?

\subsubsection{Influencia del entorno comunitario y de la red personal}

Las conversaciones en esta área están referidas al conocimiento sobre la influencia del barrio, el entorno comunitario y las relaciones en la situación problema. También explora cómo la participación de la persona en la comunidad, su vida asociativa o por el contrario, su aislamiento, están ligados al desarrollo o mantenimiento de la situación:

¿Dispone de espacios de encuentro, asociaciones, equipamientos?; ¿Cómo influye el estilo de vida del barrio en sus valores, en su identidad?; ¿Cómo se 
concreta su participación en la vida comunitaria?; ¿Su participación es valorada por la comunidad?; ¿Qué valor representa está participación para la persona?; ¿Qué le aporta?; ¿Cuáles son sus intereses?; ¿Cómo o en qué cosas ocupa su tiempo libre?, ¿Le gusta o le genera satisfacción?

Percepción social/aceptación social de la situación problema que está atravesando la persona/familia: drogas, alcohol, prostitución, malos tratos, pobreza, enfermedad mental, enfermedades raras, discapacidad, retraso escolar, marginación, delincuencia...

¿Cómo influye en la situación problema el aislamiento?; ¿Cuál es el grado de aislamiento?; ¿Cómo se relaciona con el aislamiento (bien, mal o un poco de cada)?; ¿Cuánto tiempo hace que el aislamiento se asentó en su vida?; ¿Qué lo motivo?; ¿Alguien sabe de su aislamiento?

\subsubsection{Influencia de la red profesional en el mantenimiento y/o el cambio de la situación}

Hace referencia a las conversaciones sobre las relaciones establecidas con los diferentes profesionales (eco-mapa) en el momento actual o aquellas que en algún momento del pasado les ayudaron, al tipo de vínculo establecido y la influencia que supuso en sus mejoras o en el mantenimiento de la situación problema:

¿Qué mensajes emitidos por los profesionales, recuerda la persona/familia que han contribuido a su identidad?; ¿Qué tipo de relación mantiene con los profesionales y los servicios (Sistemas Más Amplios que la Familia-SMAF)) con los que actualmente están conectados?; ¿Cómo influye la ecología profesional en el mantenimiento del problema o por el contrario, en el desarrollo de sus competencias para el cambio?; ¿Cómo influye en la vida de la persona/ familia, la intervención y la relación que mantienen entre ellos, los diferentes profesionales que están presentes en su vida?.

\subsubsection{Economía familiar}

Conocimiento sobre la organización del presupuesto familiar, el uso y el valor que se le otorga al dinero.

¿Los ingresos familiares permiten cubrir las necesidades básicas para su supervivencia y desarrollo?; Dichos ingresos, ¿permiten una seguridad familiar?; ¿Cómo se organiza el presupuesto familiar?; ¿Existe una dependencia de los servicios para subsistir?; ¿Qué significado tiene el dinero en la unidad familiar?; ¿Quién gestiona el dinero en la casa? 


\subsubsection{La vivienda como espacio relacional}

Hace referencia a las conversaciones mantenidas sobre la vivienda familiar y también a las impresiones compartidas con la persona/familia a raíz de una visita a domicilio, ligando la vivienda como su espacio relacional, así como la vivienda como derecho social.

Se busca conversar a cerca de la vivienda: ¿Es un espacio que facilita o entorpece la intimidad (hacinamiento)?; ¿Cuál es el régimen de tenencia de la vivienda?; $i$ El espacio tiene condiciones de habitabilidad (precariedad, chabolismo)?; ¿dicha vivienda está adaptada a las condiciones físicas de las personas?; ¿Su ubicación permite el contacto fluido con el barrio/la comunidad? También es importante conocer las políticas sociales del municipio sobre las viviendas y las posibilidades o impedimentos para el acceso a disponer de ella.

\subsubsection{Trabajo, ocupación, capacitación laboral y competencias}

Referido no sólo a los datos sociológicos sobre si tiene o no trabajo y la duración del mismo, sino también al trabajo como fuente de reconocimiento o frustración. Las conversaciones versarían sobre el conocimiento sobre la situación laboral de los miembros de la familia, su relación con el mercado laboral, la cualificación profesional, la formación, el nivel de instrucción, las capacidades y habilidades para el desarrollo de determinados trabajos, si existe alguna discapacidad y/o impedimento y cómo lo gestionan.

En un nivel más relacional, conocer ¿Qué significa para la persona el trabajo que está realizando?; ¿Un medio únicamente de subsistencia?; ¿Es un espacio en donde puede desarrollar sus destrezas y habilidades?; ¿Encuentra reconocimiento y apoyo, o por el contrario, es un espacio de discriminación y aislamiento?

3.2.14 Grado de motivación para el cambio y expectativas de éxito para superar/ gestionar la situación

Referido a las conversaciones que buscan compartir la expectativa de éxito en relación al proceso de cambio (Bandura, 1999).

¿Qué expectativas de éxito tiene en que su situación pueda mejorar?; ¿Qué nivel de confianza tiene en poder salir de la situación que atraviesa?; ¿Qué experiencias de éxito y fracaso lleva a sus espaldas?; ¿Cree que puede hacer algo para que la situación cambie o mejore?; ¿Es consciente del esfuerzo y motivación mantenida que necesitará para cambiar o mejorar su situación?; ¿Cuál es su nivel de esperanza en sí mismo, en su entorno relacional y en la relación de ayuda? 
3.2.15 Relación que mantiene la persona/familia con la salud y la enfermedad

Por un lado, se refiere a las conversaciones construidas sobre la relación de la persona con la salud. Identificando las pautas, por pequeñas que sean, que conforman su estilo de vida saludable.

Por otro lado, están las conversaciones construidas sobre la historia relacional de la enfermedad, no solo como algo que afecta a lo individual y subjetivo, sino cómo ésta, impacta en sus relaciones cercanas y sociales, en la familia, los amigos y la sociedad:

¿Cómo la persona /familia vive la enfermedad en su vida cotidiana?; ¿Cómo llegó la enfermedad a su vida?; ¿Qué interrumpió o no dejó iniciar?; ¿Cómo se relaciona la persona con lo que le pasa a su cuerpo?; ¿Cómo lo vive?; ¿Cómo influye el tratamiento en su vida?; ¿Cómo influye la vivencia de la enfermedad en su energía vital, en sus relaciones, en su estilo de vida, en sus aspiraciones, metas, sueños?; ¿Qué impacto tiene la enfermedad en la familia, en los amigos, en el trabajo?; ¿Cuál es su grado de dependencia psíquica y física?; ¿Cómo lo vive?; ¿Es una situación circunstancial, crónica, terminal?; ¿Qué lugar ocupan la vergüenza y la envidia?; ¿Son vividos como elementos que facilitan o que bloquean la interacción social con las personas «sanas»?; ¿Puede compartir estas emociones con su familia, amigos, profesionales de la salud?; ¿Necesita cuidadores externos?; ¿Cómo vive la dependencia?; ¿Qué expectativas y deseos le acompañan en su vida en estos momentos?; ¿Con que equipos de personal sanitario cuenta?; ¿Cuál es el estigma social de la enfermedad que padece?.

\section{Conclusiones}

La relación de ayuda colaborativa se construye en un proceso de conversaciones en el cual no hay imposiciones de planes de trabajo y no caben etiquetajes ni categorizaciones de problemas. Las conversaciones que contemplamos significan la creación de un marco de descubrimiento mutuo. Los Trabajadores/as Sociales son co-participes en la creación de procesos generadores de nuevos significados y de verdades alternativas donde la identidad de las personas es descubierta y visualizada desde sus propios saberes.

A diferencia del diagnóstico clásico, el diagnóstico relacional y colaborativo es tejido y co-construido con el otro. Profesionalmente implica generar conscientemente un espacio de apertura, dar cabida a la voz y los significados del otro. Implica no sólo un cambio de lenguaje y manera de pensar, también implica un cambio en las formas de hacer en la práctica cotidiana. En definitiva se trata, desde nuestro punto de vista, de recuperar la esencia de la práctica 
refinada con personas y familias, al tiempo que nos ubicamos en los desarrollos de las vanguardias de un trabajo social relacional y cooperativo.

\section{Bibliografía}

Andersen, T. (1994). El Equipo Reflexivo. Diálogos y diálogos sobre los diálogos. Barcelona: Gedisa.

Anderson, H. (1997). Conversation, language, and possibilities: A postmodern approach to therapy. New York: Basic Books. (Edición en castellano: Anderson, H. (1999). Conversación, lenguaje y posibilidades: Un enfoque posmoderno de la terapia. Buenos Aires: Amorrortu).

Anderson, H. y Goolishian, H. (1996). El experto es el cliente: La ignorancia como enfoque terapéutico. En McNamee, S. y Gergen, K. (Ed.). La terapia como construcción social. Barcelona: Paidós.

Arias Astray, A. y Cury, S. P. (2016). Hacia una definición actual del concepto de Diagnóstico Social. Breve revisión bibliográfica de su evolución. Alternativas. Cuadernos de Trabajo Social, 23, 9-24.

ARNKILl, T.E. y SEIKKULA, J. (2016). Diálogos terapéuticos en la Red Social. Barcelona: Herder.

BAndura, A. (1999). Auto-Eficacia: cómo afrontamos los cambios de la sociedad actual. Bilbao: Desclée de Brouwer.

Bertolino, B. y O’Hanlon, B (2001). Desarrollar Posibilidades. Barcelona: Paidós. BOLAÑOS, I. (2008). Hijos alineados y padres alienados. Madrid: Reus.

CARdona,J. (2012). La definición del contexto de intervención en el Trabajo Social de Casos. Tesis Doctoral. Universidad de las Islas Baleares.

CARdona, J. y CAmpos, J.F. (2009). Cómo determinar una contexto de intervención: Inventario para el análisis de la relación de ayuda entre el trabajador/a social y el cliente en la fase de estudio y evaluación de la situación problema. Portularia, Vol. IX, No 2, 2009, pp. 17-35.

Casement, P.(1989). Apprendere dal paziente. Milano: Raffaello Cortina Editore.

DE SHAzER, S. (1982). Patterns of Brief Family Therapy: An Ecosystemic Approach. New York: The Guilford Press.

DE SHAzER, S. (1985). Keys to Solution in Brief Therapy. New York: W W Norton \&

Company. (Edición en castellano: de Shazer, S. (1990). Claves para la Solución en Terapia Breve. Barcelona: Paidós).

DE SHAZER, S. (2009). En un origen las palabras eran magia. Barcelona: Gedisa.

DERRIDA, J. (1971). De la gramatología. Buenos Aires: Siglo XXI.

DERRIDA, J. (1989). La escritura y la diferencia. Barcelona: Anthropos.

Díaz Herráiz, E., Y FERnÁNDEZ DE CASTRO, P. (2013). Conceptualización del diagnóstico en Trabajo Social: necesidades sociales básicas. Cuadernos de Trabajo Social, 26(2), 431-443. https://doi.org/10.5209/rev_CUTS.2013.v26.n2.39550 
ESCUDERO, V. y LÓPEZ, S. (2003). Familia, evaluación e intervención. Madrid: Ed. CCS

FEYERABEND,P. (2003). Tratado contra el método. Madrid: Técnos.

FOUCAUlT, M. (2005). Vigilar y castigar. Madrid: S. XXI.

FREEMAN,S. (2001). El nuevo lenguaje del cambio. La colaboración constructiva en psicoterapia. Barcelona: Gedisa

FreEMAN, S. (Comp).(2005). Terapia familiar con equipo de reflexión. Buenos Aires: Amorrortu.

Friedlander, M., EsCUdero,V. y HeATHERINGTON, L. (2009). La alianza terapéutica. Barcelona: Paidós.

Glasser, W. (1975). Reality Therapy: A New Approach to Psychiatry. New York: Colophon Books.

GOOLISHIAN, H. Y ANDERSON, H. (1990). Understanding the therapeutic process: From individuals and families to systems in language. En F. Kaslow (Ed.). Voices in Family Psychology. Newbury Park, CA: Sage Publications.

Habermas, J. (1982). Conocimiento e interés. Madrid: Taurus.

LiPCHIK, E. (2005). Terapia centrada en soluciones. Más allá de la técnica: el trabajo con las emociones y la relación terapéutica. Buenos Aires: Amorrortu.

Madigan, S. (2011). Narrative therapy. N.Y: American Psychological Association.

MADSEN, W. C. (2007). Collaborative Therapy with Multi-Stressed Families. New York: The Guilford Press.

MASLOW, A. (1954). Motivation and Personality. New York: Harper. https://doi. org/10.1111/j.1467-6494.1954.tb01136.x

Maturana, H. y Varela, F. (2004). De máquinas y seres vivos. Autopoiesis: La organización de lo vivo (6. ${ }^{\mathrm{a}}$ Ed.). Buenos Aires: Lumen Humánitas.

MORIN, E. (1981). El método. Madrid: Cátedra.

MORIN, E. (2009). Introducción al pensamiento complejo. Madrid: Gedisa.

Morgan, A. (2004).What is narrative Therapy? An eassy-to-read introduction. Adelaide, Australia: Dulwich Centre Publications.

Selekman, M. (1993). Pathways to change. New York: Guilford Press. (Edición en castellano: Selekman, M. (1996). Abrir caminos para el cambio. Barcelona: Gedisa).

Piaget, J. (1978). Introducción a la epistemología genética. Buenos Aires: Paidós.

PiAgET, J. (1971). Estructuralism. NY: Rouledge and Kegan Paul.

RodríGUEZ RodrígUEZ, A. (2013). La intervenció sociofamiliar com a pràctica reflexiva. Revista de Treball Social. Col-legi Oficial de Treball Social de Catalunya, n. ${ }^{\circ} 198,9-18$.

Rubin, B. y BLOCH, E. (2001). Intervención en crisis y respuesta al trauma. Teoría y práctica. Bilbao: Desclée de Brouwer.

WALSH, F. (2005). Resiliencia familiar: estrategias para su fortalecimiento. Buenos Aires: Amorrortu.

Alternativas. Cuadernos de Trabajo Social, 24, 2017, pp. 67-90 - ISSN 1133-0473

DOI: 10.14198/ALTERN2017.24.05 
White, M. y Epston,D. (1990). Narrative Means to Therapeutic Ends. New York: WW Norton \& Company. (Edición en castellano: White, M. y Epston, D. (1993). Medios narrativos para fines terapéuticos. Barcelona. Paidós).

White, M. (1995). Re-Authoring Lives: Interviews and Essays. Adelaide, South Australia: Dulwich Centre Publications. (Edición en castellano: White, M. (2002). Reescribir la vida. Barcelona: Gedisa).

White, M. (2007). Maps of narrative practice. NY: W.W. Norton. (Edición en castellano: White, M. (2016). Mapas de la práctica narrativa.. Santiago de Chile: Pranas Chile Ediciones). 
Cita bibliográfica: Fernández Borrero, M. A., Relinque Medina, F. y Martí García, S. (2017). Percepciones para la intervención social desde la competencia intercultural de profesionales [Perceptions for social intervention from professionals intercultural competence]. Alternativas. Cuadernos de Trabajo Social, 24, 91-118. https://doi.org/10.14198/ ALTERN2017.24.06

\title{
PERCEPCIONES PARA LA INTERVENCIÓN SOCIAL DESDE LA COMPETENCIA INTERCULTURAL DE PROFESIONALES
}

\section{PERCEPTIONS FOR SOCIAL INTERVENTION FROM PROFESSIONALS' INTERCULTURAL COMPETENCE}

\author{
MANUELA-ÁNGELA FERNÁNDEZ BORRERO \\ Departamento de Sociología, Trabajo Social y Salud Publica de la Universidad de Huelva. \\ Facultad de Trabajo Social \\ Manuela.fernandez@dstso.uhu.es \\ FERNANDO RELINQUE-MEDINA \\ Departamento de Sociología, Trabajo Social y Salud Publica de la Universidad de Huelva. \\ Facultad de Trabajo Social \\ Fernando.relinque@pi.uhu.es \\ SUSANA MARTÍ-GARCÍA \\ Departamento de Sociología, Trabajo Social y Salud Publica de la Universidad de Huelva. \\ Facultad de Trabajo Social \\ Susana.marti@dstso.uhu.es
}

\begin{abstract}
Resumen
En este trabajo mostramos un análisis de las respuestas dadas por profesionales de los servicios sociales comunitarios a supuestos prácticos relacionados con la intervención profesional en contextos de diversidad cultural. Se pretende conocer si las respuestas a estos supuestos se relacionan con características de la intervención profesional, rasgos personales y con variables como la Competencia Intercultural y la Sensibilidad Intercultural. Han participado 253 profesionales (psicólogos, trabajadores sociales y educadores sociales). Se han realizado análisis de varianza, asociaciones con contingencia, correlaciones y Análisis Factorial de Correspondencias. Los resultados muestran que la profesión no influye en dar una respuesta adecuada a los supuestos. En cambio, tener formación en diversidad y contacto con personas de origen extranjero presentan asociaciones significativas con responder adecuadamente a ciertos supuestos. Hemos obtenido una tipología de cuatro clases diferenciadas según las respuestas, dos positivas respecto a la resolución de los supuestos y su vinculación con la competencia intercultural y dos con carácter negativo.
\end{abstract}

Palabras clave: competencia intercultural, servicios sociales, análisis de casos, intervención social, sensibilidad intercultural

\begin{abstract}
This work analysizes the responses of professionals to community social services practical cases related to professional intervention in contexts of cultural diversity. We want to know if these responses relate to the characteristics of professional intervention, personal traits and variables such as Intercultural Competence and Intercultural Sensitivity. 253 professionals have participated (psychologists, social workers and community workers). We have conducted variance analysis, measures of associations for contingency tables, correlations and Factorial Correspondence Analysis. The results show that the profession is not related to an appropriate response to the cases. Instead, training on diversity and contact with people of foreign origin has significant associations with appropriately response to certain assumptions. We have obtained a typology of four distinct classes according to responses; two positive classes regarding case resolution and their connection with intercultural competence, and two negative classes.
\end{abstract}

Keywords: intercultural competence, social services, case studies, social intervention, social 


\section{Extended abstract}

\section{Introduction}

Modern societies are culturally diverse and plural where different cultural groups living together within a shared social and political framework (Skelton y Allen, 1999). Beyond the diverse conformation of society, these flows and migration processes have also had a considerable impact on public services, especially in the medical field, which demands effective adjustments to different users and their needs. Providing culturally appropriate services is important for the development of societies and is one of the major challenges to be met by public services and professionals of social intervention (Martinez, Martinez and Calzado 2006).

In order to understand Spanish reality, it is necessary to know that access to public welfare services by foreign population is granted by Royal Decree $844 / 2013$, amending the regulations of the Organic Law 4/2000 on the rights and freedoms of foreigners in Spain. However, each region implements it in a particular way because, at the policy level, Autonomous Communities have transferred competences in social services (Andalusia - law 2/1988 of 4 April).

It is important to consider the characteristics of good intercultural practices in the provision of services based on universal access, subject active participation, duration in time, ongoing assessment and monitoring and facilitating social cohesion (Vázquez, Fernández \& Fernández-Borero, 2010:305).

\section{Professionals of Community Social Services in contexts of cultural diversity}

We consider it important that professionals ask themselves about the cultural conceptions from which they analyze the situations and demands that reach community social services, as much as about the evaluation, diagnosis and approaches to intervention strategies as they incorporate the ethical and professional notions involved and and necessary for each case.

In this paper, the starting point are the dimensions of the IC assumed as relevant for professionals in community social services (Martinez et al., 2006) with a number of practical scenarios developed by our research team.

The construction of these practical cases is the result of the combination of several elements: the training and professional practice of Social Work and Psychology of the members of the research team; the literature reviewed; and the joint and participative consultation and construction with professionals of Community Social Services. They are therefore, real cases adapted for study.

We aim to answer questions such as: is responding appropriately to cases associated with having high levels of IC?; what dimensions establish associations, or which ones gain more relevance in providing a higher number of correct answers to each of the scenarios presented?, Are there differences due to the personal characteristics of participants that may require appropriate responses to cases? The aim of this study was to determine the associations between the answers to the practical cases with professional features, the self-assigned IC level and IS. Also, we try to know if differences arise from professional practice, having different levels of IC or different socio-demographic characteristics (this is not clear, which are the terms the differences between which are to be confirmed?) responding adequately to the practical cases presented. Our last goal is drawing basic profiles depending on the variables under study.

\section{Methods}

\section{Participants}

This work has involved 253 individuals from the different professions that constitute the core team in Andalusian community social services Social Work, Psychology and Social Education.

\section{Instrument}

The instrument contains different scales:

Sociodemographic questions

A scale with nine practical cases of professional performance that must be assessed as right or wrong

A five-item Likert scale to assess the dimensions of self-attributed IC described by Martínez et al. (2006)

Another five-point Likert scale on issues of professional intervention with persons of foreign origin (Martínez et al 2006)

We also include the Spanish translation (Vila, 2005) of Chen and Starosta IS Scale (2000), with 24 items and five dimensions: involvement, respect, trust, enjoyment and attention.

\section{Analysis strategy}

We conducted an initial basic characterization of the participants in the different scales.

The main analyses were based on practical cases and related skills; we calculated correlations (Pearson $\chi 2$ ), associations, mean comparisons and variance analysis. Cohen's d (Cohen 1988) was used to calculate effect sizes. Finally, we carried ot an a Multiple Correspondence Factor Analysis (CFA).

We used the statistical software SPSS 20.0. The Anaconda software belonging to the group Catalyse tools was also used, combining factorial correspondence analysis and ascending hierarchical classification. 


\section{Results}

The accuracy rate of the cases was variable, ranging from $15,8 \%$ (case three) to $94.3 \%$ (case four), with an average level of correct answers of 4 out of the total 9 cases presented. 42.1 of the participants are at a low level of correct responses (three or less), 48.9 percent at a medium level (four to six right answers) and the remaining 9.1 percent, at a high level (seven or more properly answered cases).

The profession does not produce significant differences in the average number of cases answered correctly. Having training in cultural diversity is significantly associated with responding appropriately to the sixth case, related to preserving the professional's personal information from users and reflecting the ability to socialize and the proper management of social distance.

\section{Results on intercultural competece}

In general, terms, the overall IC is high, particularly at the conscious level, with a remarkably higher level in psychologists. In the dimensions of awareness and motivation to find out the highest competence (unconscious), social educators stand out against other professionals. In the other dimensions (knowledge, skills and encounter), the three professions show similar levels. There are no statistically significant differences between professions.

Globally IC is associated with the second case (refusal to handshake the professional with his/her left hand), so that the difference between responding properly or not occurs at the highest levels of competence; with the highest level (unconscious competence) giving correct answers; and somewhat lower levels-although high (conscious competence)-responding erroneously. Association also occurs with the eighth case, establishing the association between the level of intercultural incompetence and inappropriate response.

In matters relating to the intervention, diversity management skills stand out positively, while the lower mean shows in self-awareness of one's own biases and prejudices.

Moreover, IS is quite high ( $\mathrm{M}=4.1, \mathrm{SD}=0.44)$, with a significantly high respect for cultural differences. The total correct responses to cases not correlated with scores in global IS or in each of its dimensions.

\section{Typological analysis of results}

In this analysis, each of the axes is determined largely by a variable or a set of them. Levels of IC competence and incompetence dimensions are distributed along axis 1 , but it is also associated with IS levels. Axis 2 is related in a more direct way with the practical cases.
Aspects of professional intervention are distributed along axis 3. The results show a total of four classes.

Class 1 includes most psychologists. They are individuals with knowledge of other languages and daily contact with foreigners. They have skills in intervention and perceive the cultural diversity of the people and situations in which they work. They also know how to implement projects in these contexts and have knowledge about intercultural praxis. In addition, this class combines the most appropriate answers to cases.

Class 2. It is characterized by the highest level of IC competence in all dimensions (except for motivation). Provides a proper response to three cases (second, fourth and ninth), as well as the presence of high IS levels

Class 3 is characterized by neutrality in aspects of intervention in intercultural contexts. They have been trained in diversity at a level of incompetence in the dimension of intercultural awareness, but of competence in the motivational dimension of IC.

Class 4 shows IC levels of incompetence and IS medium levels. They are far from giving the correct answers to practical cases. In addition, they have no contact with foreigners and cannot speak other languages.

\section{Discussion and conclusions}

The results reveal some significant associations between responses to practical cases and the key variables of the study. Regarding characterization, no differences occur in the number of cases adequately answered by the different professions.

There are other matters of personal characterization that stand out, such as having training on cultural diversity, and contact with people of foreign origin. Having a certain level of IC is associated, in some cases, to successful professional interventions.

Adequate responses to a high number of cases are associated to professionals with higher intercultural awareness and a greater motivation for intercultural encounters.

The classes extracted revealed the existence of key issues in providing appropriate responses to cases that relate with high IC levels in the different dimensions considered as well as high IS levels that render key aspects for the development of professional practice in contexts of cultural diversity.

These issues can and should be dealt with the professionals of Community Social Services, including all the professions present in them, since no significant differences were found depending on profession. Training proposals can be developed to improve and tailor services and professional IC itself. 


\section{Introducción}

Los movimientos migratorios han sido muy relevantes para la construcción y conformación de la diversidad sociocultural. Han adquirido una gran importancia en Europa y en España en las últimas décadas del siglo XX y primera década del siglo XXI.

Las sociedades actuales son culturalmente diversas y plurales donde diferentes grupos culturales viven juntos dentro de un marco social y político compartido, como afirman Skelton y Allen (1999). Estos flujos y procesos migratorios han tenido también un considerable impacto en los servicios públicos, destacando el ámbito socio-sanitario, que demanda reajustes que permitan dar respuestas eficaces a los diferentes tipos de usuarios y necesidades. Disponer de servicios culturalmente adaptados es importante para el desarrollo de las sociedades y es a la vez, según Martínez, Martínez y Calzado (2006), uno de los principales retos a los que tienen que enfrentarse los servicios públicos y los profesionales de la intervención social.

Para entender la realidad española es necesario saber que el acceso a los servicios públicos de bienestar por parte de población extranjera queda reconocido por el Real Decreto 844/2013 por el que se modifica el reglamento de la Ley Orgánica 4/2000 sobre derechos y libertades de los extranjeros en España, aunque cada comunidad autónoma lo establece de un modo concreto dado que, a nivel normativo, son las Comunidades autónomas las que tienen transferidas las competencias en servicios sociales (la Ley 9/2016, de 27 de diciembre, de Servicios Sociales de Andalucía.).

Dentro de estos servicios sociales existen dos niveles: especializados, dirigidos a sectores particulares de población, y comunitarios, dirigidos a toda la población, siendo el primer nivel de atención y protección social.

Cuentan con una oferta específica de prestaciones sociales, un ámbito específico que es el de las necesidades sociales, y unos principios filosóficos que son: responsabilidad pública, solidaridad, igualdad y universalidad, participación ciudadana, descentralización, planificación/coordinación, interdisciplinariedad, normalización, globalidad y prevención. Sus funciones más importantes son adaptar los programas al contexto social, promover la participación, realizar acciones integrales y ser puerta de entrada al sistema.

El $6 \%$ del total de las personas usuarias de estos servicios son extranjeras (según datos de 2011 disponibles para Andalucía), siendo servicios referentes de primera entrada a otros sistemas sociales y dinámicas de inserción para muchas personas extranjeras; por este motivo, creemos que resulta necesaria 
la revisión de las respuestas y prácticas que se están desarrollando a la luz de la competencia intercultural (CI) de los profesionales de estos servicios.

\section{Competencia Intercultural (CI) y Servicios Sociales Comunitarios}

La noción de CI se entiende como la capacidad de dar respuestas satisfactorias en procesos interculturales incorporando comportamientos, actitudes y conocimientos que ayuden a la interacción social. En el marco de los servicios sociales, además de esta dimensión interactiva, la CI también implica el desarrollo de políticas institucionales que permitan a los profesionales ser eficaces y efectivos en situaciones de diversidad cultural. Se trata de ir más allá de una visión comprensiva de la diversidad hacia una efectiva praxis profesional intercultural. Se trataría de superar progresivamente el enfoque únicamente etic (desprovisto de cualquier intento de descubrir el significado que los agentes involucrados le dan a su realidad y acciones, con un punto de vista externo), incluyendo la perspectiva emic (que presenta la perspectiva interna al desglosar la interpretación del significado, con sus reglas y categorías, como el conocimiento sociocultural que rige y es común para un grupo o personas). Esto conduce a lo que Berry, Pootinga, Segall y Dasen. (2002: 291) denominan como «etics derivados» con gran validez intercultural, o considerando un enfoque etic flexible, entendido por Dumas et al. (1999: 182) como asumir una visión etic en la concepción pero siendo consciente y considerando la perspectiva emic existente. Es importante tener en consideración las características de las buenas prácticas interculturales en la prestación de servicios basada en el acceso universal, la participación activa de los sujetos, el mantenimiento en el tiempo, la evaluación y monitorización continua y el favorecimiento de la cohesión social (Vázquez, Fernández y Fernández-Borrero, 2010: 305).

En la lógica de la atención de los servicios culturalmente adaptados y competentes algunos aspectos básicos a considerar recogidos en la literatura son: el uso apropiado del idioma y el lenguaje, asegurando que sea comprensible y favoreciendo la capacidad de respuesta y equilibrio en las interacciones; el conocimiento de los valores y creencias presentes en la relación profesional, como puede ser el hecho de trabajar con el miedo a las consecuencias legales y burocráticas de minorías culturales; conocer las múltiples situaciones posibles derivadas de los procesos migratorios, así como las expectativas existentes sobre la relación profesional a establecer y servicios a recibir.

La CI se manifiesta cuando los profesionales entienden, aprecian, reconocen y respetan las diferencias, siendo capaces de ajustar sus prácticas y 
organizaciones al desarrollo de intervenciones efectivas en contextos de diversidad (Dana y Allen, 2008, Balcazar, Suarez-Balcazar y Willis, 2010). Se caracteriza, según Kohli, Huber y Faul (2010), por la aceptación y respeto por las diferencias, la consideración de las dinámicas diferenciales de funcionamiento, la expansión del conocimiento cultural y variedad de adaptaciones de los modelos de ayuda e intervención que permitan proveer servicios apropiados. Estos modelos pueden estar basados en la nueva lógica ecológico-sistémica del trabajo social que, según Ungar (2002), mantiene que la existencia de la diversidad social y cultural es un potencial para que el trabajo social genere nuevas soluciones para los problemas a los que se enfrenta, permitiendo emerger nuevos cambios profesionales y personales.

Los profesionales de los Servicios Sociales Comunitarios en contextos de diversidad cultural

Consideramos relevante que los profesionales se planteen desde qué concepciones culturales analizan las situaciones y demandas que llegan a los servicios sociales comunitarios, así como la evaluación, el diagnóstico y el planteamiento de la (s) estrategia(s) de intervención incorporando las nociones éticas y profesionales implícitas y necesarias en cada caso. Se trata de asumir el enfoque intercultural para ir más allá del reconocimiento de la diferencia del otro hasta lograr relaciones culturalmente más simétricas. Esta misma lógica conforma algunos de los principios deontológicos básicos de las profesiones fundamentales de los equipos base de los servicios sociales comunitarios: trabajo social, educación social y psicología. En este sentido, Garran y Werkmeister (2013) realizan una reflexión basada en los estándares establecidos en el año 2001 por la Asociación Nacional de Trabajadores Sociales de Estados Unidos, sobre la práctica culturalmente competente en un marco de compromiso profesional por la diversidad y la justicia social, y bajo la lógica de la interseccionalidad que implica comprender la diversidad cultural como un contexto más amplio de interconexiones de diversidades (género, étnica, sexual...), de influencias de poder, posicionamientos y elementos de fuerzas socio-político-estructurales que existen en cada sociedad. Implica tener una visión de la persona en cuanto a sus características individuales y sus circunstancias vitales (grupos a los que pertenece, elementos familiares, etc.), comprendiendo además que la cultura es un ingrediente de toda acción y persona en cualquier situación.

Los Standards for Cultural Competence in Social Work Practice (NASW, 2001) reflejan un avance del compromiso deontológico con la obligación 
profesional de prestar servicios culturalmente competentes, respondiendo con eficacia y bajo dos principios fundamentales: el respeto y el valor de las personas. A modo de decálogo, estos estándares recogen: 1) el desarrollo profesional de acuerdo con los valores, la ética y las normas de la profesión; 2) la comprensión de los propios valores apreciando la importancia de las identidades multiculturales; 3 ) disponer de conocimiento y la comprensión especializada sobre los elementos de las diferentes culturas; 4) disponer de habilidades interculturales, utilizando el marco metodológico y técnicas más apropiadas que reflejen una adecuada comprensión de la función de la cultura en el proceso de ayuda; 5) poner en práctica un buen servicio y asesoramiento útil; 6) desarrollar empoderamiento; 7) abogar por la diversidad y enriquecimiento dentro de la profesión y en los diferentes servicios y proyectos; 8) promover y participar en los programas de educación y capacitación que ayudan a avanzar en la competencia cultural; 9) realizar un esfuerzo por proporcionar información, referencias y servicios en el lenguaje apropiado para la persona, aunque ello requiera el uso de mediadores y/o intérpretes; y 10) desarrollar liderazgo intercultural siendo capaces de comunicar información sobre los grupos y comunidades a otros profesionales.

Estos estándares guardan una estrecha relación con las bases deontológicas profesionales. A nivel internacional, el Código de Ética de la International Federation of Social Workers (IFSW, 2004) y de la International Association of Schools of Social Work (IASSW, 2004), recogen que los trabajadores sociales deben reconocer y respetar la diversidad étnica y cultural de las sociedades con las que trabajan, teniendo en cuenta las diferencias individuales, familiares, grupales y comunitarias. El Código Deontológico del Trabajo Social en España (Consejo General del Trabajo Social, 2012) establece la igualdad como principio básico que garantiza los mismos derechos y deberes compatibles con las peculiaridades y diferencias (art. 7), así como el principio de individualización que recoge la necesidad de adecuar la intervención profesional a las particularidades específicas de cada persona, grupo o comunidad.

Los profesionales de la psicología recogen también en su código deontológico la no discriminación de personas por razón de nacimiento, edad, raza, sexo, credo, ideología, nacionalidad, clase social, o cualquier otra diferencia (art. 10). La American Psychological Association (2010) recoge, en el código de conducta y principios éticos de la profesión, el principio general de respeto de la dignidad y derechos de las personas, valorando la pertenencia cultural y las diferencias individuales.

Por último, el código deontológico de la profesión de Educación Social en España publicado por la Asociación Estatal de Educación Social (ASEDES, 
2004) contempla también como principio la igualdad, sin discriminación por razón de sexo, edad, religión, ideología, raza, idioma o cualquier otra diferencia (art. 8).

Desde el enfoque de la CI, el principio de igualdad debe completarse con la sensibilidad a las diferencias culturales, lo que requiere políticas que permitan disponer del encuadre y de los medios de actuación adecuados así como de modelos de intervención que contemplen planteamientos teóricos, estrategias, métodos y técnicas eficaces en este sentido.

Según Julve y Palomo (2005), los profesionales para ser culturalmente competentes deben tener formación en diversidad, ser conscientes del propio bagaje y perspectiva cultural y de la del otro, comprender la influencia que las características culturales juegan en los procesos de intervención y actuación profesional, evitar estereotipos y prejuicios, trabajar desde el respeto, el conocimiento, la aceptación de los propios límites, emplear técnicas y métodos que permitan el acercamiento y la comprensión mutua, y sobre todo, asentar y superar la sensibilidad intercultural (SI) para avanzar hacia la CI desde el compromiso por la igualdad.

Tomamos como punto de partida en este trabajo las dimensiones de la CI asumidas como relevantes en los profesionales de los servicios sociales comunitarios (Martínez et al., 2006) con una serie de supuestos de carácter práctico elaborados por el equipo de investigación y que se asocian con comportamientos concretos.

Los profesionales de los servicios sociales comunitarios andaluces, según el análisis realizado por el equipo de investigación en el marco del proyecto «Análisis de las necesidades formativas en los centros de servicios sociales de Andalucía» (del que también se extraen los datos de este estudio), tienen elevada CI global (en el 73 por ciento de los profesionales), sobre todo, en poseer habilidades de comunicación y relación intercultural adecuadas, tener conocimiento intercultural y desarrollar encuentros interculturales positivos (Vázquez, Fernández-Borrero y Álvarez, 2014:305).

Aunque estos profesionales tienen además alta Sensibilidad Intercultural (4,1 de media de cinco puntos), la misma no se traduce en interacciones culturalmente adaptadas,. Por esta razón, es necesario conocer la intervención intercultural en contextos de diversidad, abordando las competencias interculturales que se auto-atribuyen los trabajadores de los Servicios Sociales Comunitarios de Andalucía con las respuestas dadas a supuestos prácticos que actuarían como preguntas control. Las competencias, dimensiones y supuestos se explican y detallan en la descripción del instrumento. 
La construcción de estos casos o supuestos prácticos surge de la combinación de varios elementos: la formación y práctica profesional del Trabajo Social y la Psicología de miembros del equipo investigador, de la revisión bibliográfica realizada, y de la consulta y construcción conjunta y participativa con profesionales de los Servicios Sociales Comunitarios. Se trata por lo tanto de casos reales adaptados para el estudio, que pueden darse en cualquier centro de servicios sociales comunitarios ante los que los profesionales deben emitir un juicio sobre si la actuación profesional expuesta en cada uno de ellos es o no correcta.

Pretendemos dar respuesta a cuestiones como: ¿responder adecuadamente a los supuestos se asocia con disponer de elevados niveles de CI?, ¿qué dimensiones establecen asociaciones o cuáles de ellas adquieren más peso en otorgar mayor número de respuestas correctas a cada uno de supuestos presentados?, ¿existen diferencias debidas a características personales caracterización de los participantes que conlleven respuestas adecuadas a los supuestos? El objetivo de este trabajo es conocer las asociaciones entre las respuestas dadas a los supuestos prácticos con las características profesionales, el nivel de CI auto-atribuida y SI. Igualmente pretendemos conocer si se producen diferencias derivadas de pertenecer a una u otra profesión, tener distintos niveles de $\mathrm{CI}$ o características sociodemográficas diferentes (esto no está claro, ¿entre que se producen esas diferencias que se quieren constatar? en responder adecuadamente a los supuestos planteados. Como último objetivo planteamos la extracción de perfiles básicos en función de las variables objeto de estudio.

\section{Método}

\section{Participantes}

En este trabajo han participado 253 profesionales de los servicios sociales comunitarios en Andalucía de las diferentes profesiones que forman parte del equipo base: Trabajo Social, Psicología y Educación Social. Se ha realizado un muestreo de tipo estratificado con afijación proporcional según los puestos de trabajo, distribuyendo proporcionalmente el número total de trabajadores según los perfiles profesionales. 
100 Manuela-Ángela Fernández Borrero, Fernando Relinque-Medina y Susana Martí-García

Tabla 1. Caracterización de participantes

\begin{tabular}{|c|c|c|c|c|}
\hline & $\begin{array}{c}\text { Psicología } \\
(\mathrm{n}=38) \\
\%\end{array}$ & $\begin{array}{c}\text { Trabajo Social } \\
(\mathrm{n}=163) \\
\%\end{array}$ & $\begin{array}{c}\text { Educación Social } \\
(\mathrm{n}=52) \\
\%\end{array}$ & $\begin{array}{c}\text { TOTAL } \\
(\mathrm{N}=253) \\
\%\end{array}$ \\
\hline \multicolumn{5}{|l|}{ Género } \\
\hline Masculino & 19,35 & 7,89 & 24,24 & 15,0 \\
\hline Femenino & 80,65 & 92,11 & 75,76 & 85,0 \\
\hline \multicolumn{5}{|l|}{ Edad } \\
\hline $18-24$ & 0 & 1,23 & 0 & 0,7 \\
\hline $25-34$ & 21,05 & 30,25 & 11,54 & 23,3 \\
\hline $35-49$ & 65,79 & 64,20 & 82,69 & 69,3 \\
\hline $50-64$ & 13,16 & 4,32 & 5,77 & 6,8 \\
\hline \multicolumn{5}{|l|}{$\begin{array}{l}\text { Experiencia en } \\
\text { Servicios Sociales }\end{array}$} \\
\hline Menos de 5 años & 18,92 & 20,25 & 11,54 & 16,50 \\
\hline De 5 a 10 años & 43,24 & 30,67 & 25,00 & 28,96 \\
\hline De 11 a 20 años & 27,03 & 34,36 & 48,08 & 36,70 \\
\hline Más de 20 años & 10,81 & 14,72 & 15,38 & 17,85 \\
\hline \multicolumn{5}{|l|}{$\begin{array}{l}\text { Formación diversidad } \\
\text { cultural }\end{array}$} \\
\hline Sí & 39,47 & 60,63 & 50,00 & 55,48 \\
\hline No & 60,53 & 39,38 & 50,00 & 44,52 \\
\hline \multicolumn{5}{|l|}{ Idiomas } \\
\hline Sí & 76,32 & 61,96 & 75,00 & 67,45 \\
\hline No & 23,68 & 38,04 & 25,00 & 32,55 \\
\hline \multicolumn{5}{|l|}{ Relación } \\
\hline Sí & 81,58 & 69,33 & 84,62 & 73,49 \\
\hline No & 18,42 & 30,67 & 15,38 & 26,51 \\
\hline
\end{tabular}

\section{Instrumento}

El instrumento empleado en el estudio contiene diferentes escalas:

Preguntas sociodemográficas y de caracterización profesional: edad, sexo, experiencia profesional, formación en diversidad y contactos con personas de origen extranjero.

Una escala con nueve supuestos prácticos de actuación profesional que deben ser evaluados como correctos o incorrectos (con la opción también de no 
sabe/no contesta) por parte de los participantes, estando cada supuesto ligado a una o varias competencias interculturales. Estos supuestos pretenden descubrir el grado y/o nivel de competencias interculturales que los profesionales de la intervención ponen en juego en su praxis profesional. La correspondencia entre los supuestos y las competencias asociadas a la CI que reflejan así como la argumentación de la respuesta adecuada en cada uno se describen en el apéndice A.

\section{Apéndice A. SUPUESTOS PRÁCTICOS INCLUIDOS EN LA ENCUESTA EN RELACIÓN CON LAS COMPETENCIAS CULTURALES SEGÚN AUTORES}

\begin{tabular}{|c|c|c|c|}
\hline SUPUESTOS PRÁCTICOS & $\begin{array}{c}\text { COMPETENCIAS } \\
\text { CULTURALES } \\
\text { RELACIONADAS }\end{array}$ & AUTORES & $\begin{array}{l}\text { Argumentación de la respuesta } \\
\text { adecuada }\end{array}$ \\
\hline $\begin{array}{l}\text { 1. A su lugar de trabajo } \\
\text { llega un matrimonio. El } \\
\text { hombre se sienta enfrente } \\
\text { de Ud. y la mujer se queda } \\
\text { detrás. Lo primero que } \\
\text { harías sería pedirle a la } \\
\text { señora que se sentara al lado } \\
\text { de su esposo. }\end{array}$ & $\begin{array}{l}\text { «habilidad para no } \\
\text { enjuiciar, teniendo } \\
\text { conciencia de } \\
\text { las diferencias } \\
\text { culturales» }\end{array}$ & $\begin{array}{l}\text { Isidro Maya } \\
\text { (2002) } \\
\text { Cui Geng and } \\
\text { Njoku Awa } \\
\text { (1991) }\end{array}$ & $\begin{array}{l}\text { Incorrecto porque las personas } \\
\text { que mantengan relaciones } \\
\text { o trabajen en contextos } \\
\text { interculturales deben respetar } \\
\text { y aceptar estas diferencias } \\
\text { culturales. Es incorrecto que el/ } \\
\text { la profesional anime a la señora } \\
\text { a que se sitúe al mismo nivel que } \\
\text { su marido sin conocer y/o aceptar } \\
\text { las costumbres, valores, etc. } \\
\text { culturalmente diferentes. }\end{array}$ \\
\hline $\begin{array}{l}\text { 2. A su lugar de trabajo } \\
\text { acude una persona. Usted } \\
\text { tiene la mano derecha } \\
\text { ocupada en esos momentos } \\
\text { y le extiende la mano } \\
\text { izquierda a modo de saludo, } \\
\text { pero le rechaza. Esta } \\
\text { reacción le extraña. }\end{array}$ & $\begin{array}{l}\text { «comprender y } \\
\text { conocer nuestra } \\
\text { propia cultura» } \\
\text { «comprender y } \\
\text { conocer la/s otra/s } \\
\text { cultura/s» }\end{array}$ & $\begin{array}{l}\text { Maya (2002) } \\
\text { Richard } \\
\text { Wiseman, } \\
\text { Mitchell } \\
\text { Hammer and } \\
\text { Hiroko Nishida } \\
\text { (1989) }\end{array}$ & $\begin{array}{l}\text { Incorrecto, ya que según } \\
\text { los autores relacionados, es } \\
\text { incorrecto que a los profesionales } \\
\text { les extrañe este tipo de } \\
\text { reacciones, ya que se debe a que } \\
\text { no están formados ni poseen los } \\
\text { conocimientos necesarios acerca } \\
\text { de las culturas de los usuarios a } \\
\text { los que atienden. }\end{array}$ \\
\hline $\begin{array}{l}\text { 3. La persona que va a } \\
\text { verle no habla muy bien el } \\
\text { castellano. Para solventar } \\
\text { esta cuestión, usted llama a } \\
\text { un intérprete/ mediador que } \\
\text { le ayude en la comunicación. }\end{array}$ & $\begin{array}{l}\text { «tolerar la } \\
\text { ambigüedad y la } \\
\text { incertidumbre» }\end{array}$ & $\begin{array}{l}\text { Geng and Awa } \\
\text { (1991) } \\
\text { Maya (2002) }\end{array}$ & $\begin{array}{l}\text { Incorrecto porque en situaciones } \\
\text { poco definidas e inciertas donde } \\
\text { la persona no domina muy } \\
\text { bien el idioma, el nerviosismo } \\
\text { del profesional hace que } \\
\text { recurra a otros profesionales } \\
\text { como intérpretes, traductores, } \\
\text { Siguiendo a los autores citados, } \\
\text { esto genera indefensión y } \\
\text { desconfianza en el usuario y } \\
\text { la calidad de la intervención } \\
\text { disminuye. }\end{array}$ \\
\hline $\begin{array}{l}\text { 4. Cada vez que usted } \\
\text { habla sobre métodos de } \\
\text { planificación familiar, } \\
\text { la señora china que está } \\
\text { enfrente suya, sonrie. Usted } \\
\text { cree que le está entendiendo } \\
\text { y le explica con detalle } \\
\text { algunos de estos métodos. }\end{array}$ & $\begin{array}{l}\text { «flexibilidad } \\
\text { cognitiva» } \\
\text { «empatía cultural» } \\
\text { «comprender y } \\
\text { conocer la/s otra/s } \\
\text { cultura/s» }\end{array}$ & $\begin{array}{l}\text { Maya (2002) } \\
\text { Geng and Awa } \\
(1992)\end{array}$ & $\begin{array}{l}\text { Es incorrecto que el profesional } \\
\text { continúe explicando } \\
\text { detalladamente los métodos } \\
\text { anticonceptivos debido a que } \\
\text { no está suspendiendo su juicio } \\
\text { momentáneamente, ni situándose } \\
\text { con más facilidad en la postura } \\
\text { de la usuaria, ni comprendido la } \\
\text { cultura de la señora. }\end{array}$ \\
\hline
\end{tabular}

Alternativas. Cuadernos de Trabajo Social, 24, 2017, pp. 91-118 - ISSN 1133-0473 
5. La hija de un matrimonio recién divorciado cree que su madre no debería haber dado el paso de solicitar el divorcio. Tú le explicas que eso es normal aqui y que hay muchos niños con los padres divorciados, y no pasa nada

6. En una visita a domicilio, ante sus preguntas sobre la situación familiar, la señora le pregunta a usted si está casada-o, si tiene hijos... Usted no le da esta información porque es una cuestión personal que no afecta a la intervención.

\section{Cuando le comenta} al señor que puede tener derecho a determinados recursos, él le dice siempre que lo que Dios quiera, que lo que Dios quiera... Usted le dice que no, que es algo a lo que tiene derecho.

8. La persona con la que habla está muy cerca de usted hasta el punto que intenta siempre poner la mano sobre su brazo. Usted lo retira porque se siente incómodo/a.

9. Realiza una visita a
domicilio. El señor le atiende
en el salón y la señora se
queda en la cocina. Usted,
que quiere hablar con los
dos, alza la voz para que la
señora le oiga y le responda.

«habilidad para formar y mantener relaciones» «mantener una distancia social apropiada»

«habilidad para comprender y conocer nuestra propia cultura» «habilidad para comprender y conocer las demás culturas» «flexibilidad cognitiva»

«mantener una distancia social apropiada»

«habilidad para la resolución de conflictos»
Maya (2002)

Geng and Awa (1992)

Maya (2002)

Wiseman et al (1989)

Geng and Awa (1992)

Wiseman et al (1989)

Maya (2002)

Geng and Awa (1992) Maya (2002)
Incorrecto porque no está empatizando culturalmente con la protagonista de la intervención y por consiguiente, no está poniéndose en su lugar, respetándola y aceptando su manera de ver y entender el mundo.

Es incorrecto que se responda a la usuaria con que es una cuestión personal debido a que estas competencias son desarrolladas por diversos autores mencionados como un elemento de vital importancia para identificar y gestionar las necesidades personales y el apoyo, y establecer contacto y mantener relaciones con personas extranjeras.

Adquirir conocimientos culturales (generales y específicos) ayuda a interactuar de forma positiva y eficaz con personas de diferentes culturas. Es incorrecta la actitud seguida por el/la profesional, no dejando que la persona elija libremente si desea o no los recursos a los que tiene derecho, aunque el rechazo sea por cuestiones únicamente culturales.

Incorrecto porque cada cultura da una relevancia diferente a la distancia que debe existir entre profesional y usuario. Es importante entender cuál es la distancia adecuada que puede determinar la conformación y mantenimiento de las relaciones sociales con los demás.

Incorrecto porque es necesario re conocer de manera adecuada al interlocutor si queremos tener éxito en la interacción comunicativa. Implicar a otra persona que no está próxima no solo dificulta la comunicación, sino que niega la interlocución de quien tenemos delante.

Elaboración propia

Alternativas. Cuadernos de Trabajo Social, 24, 2017, pp. 91-118 - ISSN 1133-0473 
Una escala de cinco ítems tipo Likert para valorar las dimensiones de la CI auto-atribuida, descritas por Martínez et al. (2006): grado de conciencia intercultural, deseo por el encuentro intercultural, grado de conocimiento cultural, desarrollo de habilidades culturales y encuentro intercultural. Las preguntas son valoradas a partir de una graduación de cuatro posibilidades (Purnell y Paulanka, 1998), donde el uno responde a la incompetencia inconsciente y el sujeto muestra ceguera cultural al no tener constancia de su falta de conocimientos, conciencia, etc.; el dos al de incompetencia consciente que implica que la persona percibe su hándicap cultural pero sus conocimientos al respecto son escasos; tres a competencia consciente que indica que el sujeto hace un esfuerzo voluntario por hacer suyos ciertos conocimientos y pautas culturales con respecto a su cultura y a las otras y es capaz de ofrecer respuestas profesionales culturalmente sensibles; y cuatro a competencia inconsciente como habilidad interiorizada (prácticamente automatizada) para prestar servicios y ofrecer respuestas culturalmente sensibles según las características de cada sujeto.

Otra escala tipo Likert de grado de acuerdo de cinco puntos sobre aspectos de intervención de los profesionales con personas de origen extranjero, tomada de Martínez et al. (2006), que incluye: ser consciente de los propios sesgos y prejuicios hacia grupos culturales diferentes; poseer habilidades para gestionar los aspectos de diversidad en las situaciones profesionales; tener conocimientos para percibir los aspectos de diversidad en estas situaciones profesionales; tener los conocimientos necesarios para diseñar un proyecto de intervención teniendo en cuenta los elementos de diversidad cultural; y tener conocimientos suficientes sobre los elementos de diversidad de las personas usuarias.

Se incluye también la traducción en castellano de la Escala de SI Vilá, 2005) propuesta por Chen y Starosta (2000), de 24 ítems y cinco dimensiones: implicación, respeto, confianza, disfrute y atención.

\section{Estrategia de análisis}

Realizamos una caracterización básica inicial de los participantes en las diferentes escalas: $\mathrm{CI}$ auto-atribuida, competencias específicas y aspectos relacionados con la intervención culturalmente adecuada.

Los análisis principales se basan en los supuestos prácticos y en las competencias relacionadas, habiéndose realizado correlaciones ( $\chi^{2}$ Pearson), asociaciones, comparaciones de medias y análisis de la varianza. Se emplea la $d$ de Cohen (Cohen 1988) para el cálculo de los tamaños de efectos, considerando 
los valores de $d$ hasta 0.20 como efectos pequeños, hasta 0.50 como tamaños del efecto medianos y hasta 0.80 o más como elevados.

Finalmente se realiza un Análisis Factorial de Correspondencias Múltiples (AFC). Se trata de un análisis multivariante de carácter exploratorio, para la extracción de clases, que permite elaborar grupos de perfiles de los participantes según las variables claves del estudio.

Para el análisis y tratamiento de los datos empleamos el software estadístico SPSS 20.0. También utilizamos el software Anaconda pertenecientes al grupo de herramientas Catalyse, que permite hacer un análisis cuantitativo de los datos, combinando el análisis factorial de correspondencias y la clasificación jerárquica ascendente. Este grupo de herramientas Catalyse surgen del trabajo de la Red Europea de Inteligencia Territorial ${ }^{1}$ y se emplean para el desarrollo de diagnósticos y evaluación de datos individuales para definir perfiles o tipologías, que permitan ofrecer visiones conjuntas de datos y variables.

\section{Resultados}

Inicialmente se muestran los resultados descriptivos básicos para los supuestos y los análisis de varianzas y asociaciones entre estos supuestos y las características sociodemográficas. Presentamos igualmente las asociaciones, correlaciones y diferencias significativas en relación con los supuestos de las variables claves del estudio como la CI, competencias de intervención intercultural y SI.

\section{Análisis de los supuestos estudiados}

El índice de acierto de los supuestos ha sido variable, oscilando entre el 15, $8 \%$ (del supuesto tres) y el 94,3\% (del supuesto cuatro), con un nivel medio de respuestas correctas de 4 supuestos del total de 9 presentados.

Un 42,1 de los participantes se sitúan en un nivel bajo de respuestas correctas (tres o menos), un 48,9 por ciento en nivel medio (de cuatro a seis respuestas adecuadas) y el resto, un 9,1 por ciento, en nivel alto (siete o más supuestos adecuadamente respondidos).

1. European Network of Territorial Intelligence (ENTI) integra la investigación europea actual sobre las herramientas de inteligencia territorial. 
Tabla 2. Respuestas dadas a los supuestos según profesiones

\begin{tabular}{lcccc}
\hline & $\begin{array}{c}\text { Psicología } \\
(\mathrm{n}=38) \\
\%\end{array}$ & $\begin{array}{c}\text { Trabajo } \\
\text { Social } \\
(\mathrm{n}=163) \\
\%\end{array}$ & $\begin{array}{c}\text { Educación } \\
\text { Social } \\
(\mathrm{n}=52) \\
\%\end{array}$ & $\begin{array}{c}\text { TOTAL } \\
(\mathrm{N}=253) \\
\%\end{array}$ \\
\hline Respuesta adecuada (\%) & & & & \\
Supuesto 1 matrimonio & 43,8 & 24,3 & 28,3 & 26,5 \\
Supuesto 2 manos & 70,4 & 59,4 & 81,1 & 66,0 \\
Supuesto 3 mediación & 9,4 & 14,0 & 25,0 & 15,8 \\
Supuesto 4 china & 90,0 & 78,3 & 83,3 & 82,9 \\
Supuesto 5 divorcio & 53,1 & 35,2 & 31,8 & 35,8 \\
Supuesto 6 personal & 50,0 & 50,0 & 68,9 & 56,9 \\
Supuesto 7 derecho & 41,4 & 33,6 & 33,3 & 34,3 \\
Supuesto 8 cercanía & 74,1 & 52,3 & 57,1 & 59,6 \\
Supuesto 9 gritar & 93,8 & 94,2 & 92,2 & 94,3 \\
Niveles de respuestas adecuadas & & & & \\
Bajo (0-3) & 31,6 & 48,8 & 38,5 & 42,1 \\
Medio (4-6) & 60,5 & 43,2 & 48,1 & 48,8 \\
Alto (7 a 9) & 7,9 & 8 & 13,5 & 9,1 \\
\hline
\end{tabular}

La profesión no produce diferencias significativas en el número medio de supuestos respondidos correctamente, aunque los trabajadores sociales tienen 3,8 respuestas correctas de media, frente a 4,2 de los educadores sociales y psicólogos. La variable edad determina una asociación significativa entre las personas de mayor edad (de 50 a 64 años) con responder inadecuadamente al cuarto supuesto planteado, sobre la explicación de métodos de planificación familiar ante una persona extranjera china que sólo sonríe $(\chi 2(3, N=191)=$ $8,57, \mathrm{p}=0,036)$ y al quinto sobre normalización del divorcio $(\chi 2(3, \mathrm{~N}=217)=$ $8,06, \mathrm{p}=0,045)$, que reflejan la competencia de comprender y conocer otras culturas y la empatía cultural.

Tener formación en diversidad cultural se asocia significativamente con responder adecuadamente el sexto supuesto que versa sobre la preservación de la información personal del profesional ante la persona usuaria $(\chi 2(3, \mathrm{~N}=$ $194)=3,99, p=0,046)$ y que refleja la habilidad de relación y el manejo adecuado de la distancia social. Además, las personas que mantienen algún tipo de relación con personas extranjeras se asocian con la respuesta adecuada de este mismo supuesto $(\chi 2(1, \mathrm{~N}=213)=4,43, \mathrm{p}=0,026)$ y del supuesto séptimo donde el profesional explica los derechos de las personas usuarias $(\chi 2(1, \mathrm{~N}=$ $211)=3,48, p=0,043)$ mostrando la habilidad de conocer y comprender tanto la cultura propia como ajenas, así como la flexibilidad cognitiva. 


\section{Resultados sobre competencia intercultural}

En líneas generales La CI global es elevada, sobre todo a nivel consciente y destacando un nivel más alto en los psicólogos. En las dimensiones de conciencia y de motivación por el encuentro destaca la máxima competencia (inconsciente) de los educadores sociales frente a otros profesionales. En el resto de dimensiones (conocimiento, desarrollo de habilidades y encuentro) las tres profesiones muestran niveles similares. No se producen diferencias estadísticamente significativas entre las profesiones.

Tabla 3. Dimensiones (graduación) de la Competencia intercultural autoatribuida por profesiones

\begin{tabular}{|c|c|c|c|c|c|c|c|}
\hline & & $\begin{array}{c}\text { Conciencia } \\
(\%)\end{array}$ & $\begin{array}{l}\text { Motivación por } \\
\text { el encuentro } \\
\text { intercultural(\%) }\end{array}$ & $\begin{array}{c}\text { Conocimiento } \\
(\%)\end{array}$ & $\begin{array}{c}\text { Desarrollo } \\
\text { habilidades } \\
\text { (\%) }\end{array}$ & $\begin{array}{c}\text { Encuentro } \\
\text { intercultural } \\
\text { (\%) }\end{array}$ & $\begin{array}{c}\text { Competencia } \\
\text { global (\%) }\end{array}$ \\
\hline \multirow{4}{*}{$\begin{array}{c}\text { Total } \\
(\mathrm{N}=253)\end{array}$} & $\begin{array}{l}\text { Incompetencia } \\
\text { inconsciente }\end{array}$ & 29,2 & 34,2 & 1,0 & 4,1 & 6,1 & 0,7 \\
\hline & $\begin{array}{l}\text { Incompetencia } \\
\text { consciente }\end{array}$ & 14,2 & 12,4 & 27,3 & 14,9 & 28,4 & 10,7 \\
\hline & $\begin{array}{l}\text { Competencia } \\
\text { consciente }\end{array}$ & 49,5 & 15,1 & 66,0 & 62,4 & 38,5 & 64,6 \\
\hline & $\begin{array}{l}\text { Competencia } \\
\text { inconsciente }\end{array}$ & 7,1 & 38,3 & 5,7 & 18,6 & 27,0 & 24,1 \\
\hline \multirow{4}{*}{$\begin{array}{l}\text { Psicólogos/as } \\
\qquad(\mathrm{N}=38)\end{array}$} & $\begin{array}{l}\text { Incompetencia } \\
\text { inconsciente }\end{array}$ & 24,3 & 47,4 & 0 & 2,7 & 0 & 0 \\
\hline & $\begin{array}{l}\text { Incompetencia } \\
\text { consciente }\end{array}$ & 13,5 & 15,8 & 28,9 & 13,5 & 27,0 & 8,3 \\
\hline & $\begin{array}{l}\text { Competencia } \\
\text { consciente }\end{array}$ & 56,8 & 13,2 & 63,2 & 67,6 & 43,2 & 75,0 \\
\hline & $\begin{array}{l}\text { Competencia } \\
\text { inconsciente }\end{array}$ & 5,4 & 23,7 & 7,9 & 16,2 & 29,7 & 16,7 \\
\hline \multirow{4}{*}{$\begin{array}{c}\text { Trabajadores } \\
\text { sociales } \\
(\mathrm{N}=163)\end{array}$} & $\begin{array}{l}\text { Incompetencia } \\
\text { inconsciente }\end{array}$ & 32,3 & 31,3 & 1,8 & 4,3 & 6,8 & 1,3 \\
\hline & $\begin{array}{l}\text { Incompetencia } \\
\text { consciente }\end{array}$ & 16,1 & 11,7 & 27,0 & 16,8 & 31,5 & 11,9 \\
\hline & $\begin{array}{l}\text { Competencia } \\
\text { consciente }\end{array}$ & 43,5 & 17,2 & 65,6 & 59,0 & 35,2 & 62,3 \\
\hline & $\begin{array}{l}\text { Competencia } \\
\text { inconsciente }\end{array}$ & 8,1 & 39,9 & 5,5 & 19,9 & 26,5 & 24,5 \\
\hline \multirow{4}{*}{$\begin{array}{l}\text { Educadores } \\
\text { sociales } \\
(\mathrm{N}=52)\end{array}$} & $\begin{array}{l}\text { Incompetencia } \\
\text { inconsciente }\end{array}$ & 23,1 & 32,7 & 0 & 3,8 & 1,9 & 0 \\
\hline & $\begin{array}{l}\text { Incompetencia } \\
\text { consciente }\end{array}$ & 9,6 & 13,5 & 35,3 & 15,4 & 23,1 & 9,8 \\
\hline & $\begin{array}{l}\text { Competencia } \\
\text { consciente }\end{array}$ & 61,5 & 13,5 & 62,7 & 65,4 & 51,9 & 68,6 \\
\hline & $\begin{array}{l}\text { Competencia } \\
\text { inconsciente }\end{array}$ & 5,8 & 40,4 & 2,0 & 15,4 & 23,1 & 21,6 \\
\hline
\end{tabular}


Los análisis han arrojado algunas asociaciones significativas entre dimensiones de la CI con determinados supuestos. De este modo, responder adecuadamente al segundo supuesto se asocia con el mayor nivel de competencia de la conciencia intercultural $(\chi 2(3, N=194)=8,90, p=0,031)$. Además, responder adecuadamente a un número elevado de supuestos ( 7 o más) se asocia con la competencia consciente de la conciencia intercultural ( $\chi 2(6, \mathrm{~N}$ $=249)=23,29, \mathrm{p}=0,001$ ), y un nivel bajo de respuestas con la incompetencia. Los tamaños de efecto son pequeños a pesar de la significatividad estadística ( $V$ de Cramer de 0,21 y de 0,29 respectivamente).

El nivel de competencia consciente de la motivación por el encuentro intercultural se asocia significativamente con responder adecuadamente al primer supuesto, que sería calificar como incorrecto que el profesional invite a la mujer usuaria a sentarse al lado de su marido $(\chi 2(3, N=230)=9,13, p=0,018 ; \mathrm{V}$ de Cramer $=0,20)$ reflejando la habilidad de no enjuiciar. Esta elevada motivación también se asocia con el nivel alto de supuestos respondidos adecuadamente $(\chi 2(6, \mathrm{~N}=252)=15,10 \mathrm{p}=0,019 ; \mathrm{V}$ de Cramer=0,17).

El nivel máximo de competencia en conocimiento cultural se asocia de manera significativa con la respuesta adecuada al segundo supuesto, que versaba sobre un rechazo o reparo de la persona usuaria ante un modo de saludo del profesional $(\chi 2(3, \mathrm{~N}=196)=9,48 \mathrm{p}=0,024 ; \mathrm{V}$ de Cramer $=0,220)$ y que refleja la habilidad de comprender y conocer tanto la cultura propia como otras diferentes.

Por otra parte, ser incompetentes para el encuentro intercultural se asocia con responder inadecuadamente al supuesto octavo, que recoge un ejemplo de incomodidad del profesional ante una escasa distancia social de la persona usuaria $(\chi 2(3, N=197)=9,09 p=0,028 ; \mathrm{V}$ de Cramer $=0,215)$.

La dimensión de desarrollo de habilidades no se asocia significativamente con ningún supuesto.

Por último, la $\mathrm{CI}$, considerada a nivel global, se asocia con el segundo supuesto (rechazo ante el saludo con la mano izquierda del profesional) $(\chi 2$ $(3, \mathrm{~N}=192)=8,56 \mathrm{p}=0,036 ; \mathrm{V}$ de Cramer $=0,211)$, de modo que la diferencia entre responder adecuadamente o no se produce en los niveles máximos de competencia, siendo los de mayor nivel (competencia inconsciente) los que dan respuestas correctas y los que tienen un nivel algo menor, aunque elevado (competencia consciente) los que responden erróneamente. También se produce asociación con el octavo supuesto que exponía el rechazo profesional a una escasa distancia social de la persona usuaria, estableciéndose la asociación 
el nivel de incompetencia intercultural y responder inadecuadamente ( $\chi 2$ (3, $\mathrm{N}=192)=8,65 \mathrm{p}=0,034 ; \mathrm{V}$ de Cramer=0,212).

En cuestiones relativas a la intervención destaca positivamente las habilidades en la gestión de la diversidad, mientras que la menor media se presenta en la conciencia de los propios sesgos y prejuicios. Los trabajadores sociales muestran, frente a educadores sociales, mayor percepción de los aspectos de diversidad presentes en situaciones profesionales $(F(2,244)=3,43 p=0,034$, $d=.69$, IC $95 \%[.02, .77])$.

Los profesionales que responden adecuadamente al primer supuesto sobre habilidad de no enjuiciar (el profesional le pide a la mujer de un matrimonio heterosexual que se siente al lado de su marido), son significativamente más conscientes de sus propios sesgos o prejuicios $(t(218)=2,28, p=0,023, d=.58$, IC $95 \%[0,067,0,918])$ que aquellos que responden incorrectamente.

Los que responden adecuadamente al supuesto octavo, sobre la distancia social, tienen significativamente mejor puntuación en la escala global de intervención $(t(193)=2,15, p=0,033, d=.31$, IC 95\% [0,018, 0,409]) y mayor media en habilidades de gestión de la diversidad en situaciones profesionales $(t(191)=2,83, p=0,005, d=.40$, IC $95 \%[0,108,0,604])$ que aquellos que responden de manera inadecuada.

Por otra parte, la SI es bastante elevada $(M=4,1 ; S D=0,44)$, destacando un elevado respeto por las diferencias culturales (tabla 3). El total de respuestas correctas a los supuestos no correlaciona con las puntuaciones en SI global ni en cada una de sus dimensiones. Únicamente se han encontrado diferencias estadísticamente significativas en la implicación en relación con la respuesta al segundo supuesto, siendo mayor la implicación para los que responden adecuadamente $(t(141)=0,18, p=0,011, d=.46$, IC 95\% $[0,058,0,438])$. 
Tabla 4. Aspectos relacionados con competencia intercultural y Sensibilidad Intercultural

\begin{tabular}{|c|c|c|c|c|c|c|c|c|}
\hline \multirow[b]{2}{*}{ INTERVENCIÓN } & \multicolumn{2}{|c|}{$\begin{array}{c}\text { Total } \\
(\mathrm{N}=253)\end{array}$} & \multicolumn{2}{|c|}{$\begin{array}{l}\text { Psicólogos/as } \\
\quad(\mathrm{N}=38)\end{array}$} & \multicolumn{2}{|c|}{$\begin{array}{c}\text { Trabajador/a } \\
\text { social } \\
(\mathrm{N}=163)\end{array}$} & \multicolumn{2}{|c|}{$\begin{array}{c}\text { Educadores/as } \\
\text { sociales } \\
(\mathrm{N}=52)\end{array}$} \\
\hline & Mean & SD & Mean & SD & Mean & $\mathrm{SD}$ & Mean & SD \\
\hline Conciencia de prejuicios & 3,0 & 1,46 & 3,29 & 1,384 & 2,91 & 1,490 & 3,23 & 1,403 \\
\hline Habilidades gestión diversidad & 3,7 & ,90 & 3,78 & ,929 & 3,76 &, 895 & 3,52 & ,863 \\
\hline Conocimiento percibir diversidad & 3,4 & ,95 & 3,44 & ,998 & 3,50 & ,927 & 3,10 & ,931 \\
\hline $\begin{array}{l}\text { Conocimiento para diseño de proyectos } \\
\text { intervención }\end{array}$ & 3,1 & 1,10 & 3,03 & 1,158 & 3,15 & 1,115 & 2,86 & ,990 \\
\hline Conocimiento sobre las diferencias de usuarios & 3,3 & 1,02 & 3,36 & 1,175 & 3,29 & ,996 & 3,18 & 1,004 \\
\hline Competencia Intervención Intercultural (Suma) & 3,3 & ,68 & 3,33 &, 780 & 3,32 & ,668 & 3,18 & ,639 \\
\hline \multicolumn{9}{|l|}{ SENSIBILIDAD INTERCULTURAL } \\
\hline Implicación & 4,1 &, 55 & 4,1 &, 51 & 4,1 &, 59 & 4,2 &, 47 \\
\hline Respeto & 4,5 &, 54 & 4,5 & ,48 & 4,5 &, 58 & 4,6 & 41 \\
\hline Confianza & 3,7 & ,63 & 3,8 & ,69 & 3,7 & ,62 & 3,5 & ,60 \\
\hline Disfrute & 4,1 & ,88 & 4,4 &, 57 & 4,0 & ,90 & 4,1 & ,93 \\
\hline Atención & 4,0 & ,69 & 4,1 &, 75 & 3,9 &, 70 & 4,1 &, 55 \\
\hline Global & 4,1 &, 44 & 4,2 &, 33 & 4,1 &, 48 & 4,1 &, 36 \\
\hline
\end{tabular}

Análisis tipológico de los resultados

Posteriormente efectuamos un Análisis Factorial de Correspondencias, para realizar un análisis tipológico de los participantes en función de su caracterización, sus puntuaciones en las variables claves y en los supuestos.

En este análisis cada uno de los ejes está determinado fundamentalmente por una variable o conjunto de ellas. El eje 1, distribuye según los niveles de incompetencia y competencia de las dimensiones de CI, aunque también lleva asociado los niveles de SI. El eje 2 se relaciona de un modo más directo con las respuestas adecuadas a los supuestos prácticos planteados y, el eje 3, distribuye según posesión o no de los aspectos de intervención profesional. Los resultados muestran un total de cuatro clases diferenciadas, donde la primera y segunda clase son las más positivas.

La clase uno concentra la mayoría de psicólogos participantes y viene definida fundamentalmente por el eje tres. Son individuos con conocimiento de otros idiomas y con contacto cotidiano con personas extranjeras. Están de acuerdo en tener habilidades en intervención y en percibir la diversidad cultural de las personas y situaciones en las que trabajan. Saben realizar proyectos en estos contextos y disponen de conocimientos sobre la praxis intercultural. Además, esta clase aúna las respuestas adecuadas a la mayoría de los supuestos. 
La clase dos, también positiva, viene marcada fundamentalmente por el eje 1. Se caracteriza por el mayor nivel de competencia en todas las dimensiones de CI (menos la motivación). A esto se añade la respuesta adecuada a tres supuestos (segundo, cuarto y noveno), lo que le acerca a la clase uno, así como la presencia de los niveles elevados de SI, fundamentalmente de implicación, respeto y atención.

La clase tres, más asociada al eje tres, se caracteriza por la neutralidad en los aspectos de intervención en contextos de interculturalidad. Tienen formación en diversidad pero con nivel de incompetencia en la dimensión de conciencia intercultural, pero competencia en la dimensión de motivación de la CI. El nivel de respuestas adecuadas en los supuestos es bajo (4 supuestos de 9).

La clase cuatro es la que muestra los niveles de incompetencia de la CI, así como los niveles medios de SI. Se encuentran alejados de las respuestas correctas a los supuestos. Además, no tienen contacto con personas extranjeras y no tienen conocimientos de otros idiomas.

Siendo el grueso de edad de la muestra de 35 a 49 años, esta franja se localiza en mayor proporción en la clase tercera. En cuanto al sexo, en todas las clases destacan las mujeres dado que el 87 por ciento del total de la muestra son mujeres, mientras que el porcentaje de hombres se aglutina en las dos primeras clases (las más positivas).

Tabla 5. Características de identificación por clases (distribución por filas y columnas)

\begin{tabular}{|c|c|c|c|c|c|c|c|c|}
\hline & \multicolumn{4}{|c|}{$\%$ columnas } & \multicolumn{4}{|c|}{$\%$ filas } \\
\hline & $\begin{array}{l}\text { Clase } 1 \\
(\mathrm{~N}=52)\end{array}$ & $\begin{array}{c}\text { Clase } \\
2 \\
(\mathrm{~N}=73)\end{array}$ & $\begin{array}{l}\text { Clase } 3 \\
(\mathrm{~N}=81)\end{array}$ & $\begin{array}{l}\text { Clase } 4 \\
(\mathrm{~N}=47\end{array}$ & Clase 1 & Clase 2 & Clase 3 & Clase 4 \\
\hline Clase & & & & & 20,6 & 28,9 & 32 & 18,6 \\
\hline \multicolumn{9}{|l|}{ Género } \\
\hline Masculino & 18,2 & 18,0 & 2,1 & 13,9 & 34,8 & 39,1 & 4,3 & 21,7 \\
\hline Femenino & 81,8 & 82,0 & 97,9 & 86,1 & 23,2 & 26,5 & 30,3 & 20,0 \\
\hline \multicolumn{9}{|l|}{ Edad } \\
\hline $18-24$ & 0 & 1,4 & 0 & 2,1 & 0 & 50,0 & 0 & 50,0 \\
\hline $25-34$ & 31,4 & 27,4 & 24,7 & 14,9 & 25,4 & 31,7 & 31,7 & 11,1 \\
\hline $35-49$ & 56,9 & 69,9 & 67,9 & 78,7 & 16,9 & 29,7 & 32,0 & 21,5 \\
\hline $50-64$ & 11,8 & 1,4 & 7,4 & 4,3 & 40,0 & 6,7 & 40,0 & 13,3 \\
\hline \multicolumn{9}{|c|}{ Formación en diversidad } \\
\hline Sí & 61,5 & 65,3 & 55,1 & 32,6 & 23,4 & 34,3 & 31,4 & 10,9 \\
\hline No & 38,5 & 34,7 & 44,9 & 67,4 & 18,0 & 22,5 & 31,5 & 27,9 \\
\hline \multicolumn{9}{|l|}{ Relación } \\
\hline Sí & 94,2 & 82,2 & 63,0 & 59,6 & 26,1 & 31,9 & 27,1 & 14,9 \\
\hline No & 5,8 & 17,8 & 37,0 & 40,4 & 4,6 & 20,0 & 46,2 & 29,2 \\
\hline \multicolumn{9}{|l|}{ Idioma } \\
\hline Sí & 88,5 & 69,9 & 60,5 & 48,9 & 27,2 & 30,2 & 29,0 & 13,6 \\
\hline No & 11,5 & 30,1 & 39,5 & 51,1 & 7,1 & 26,2 & 38,1 & 28,6 \\
\hline
\end{tabular}


Figura 1. Distribución espacial de clases en los diferentes planos/ejes

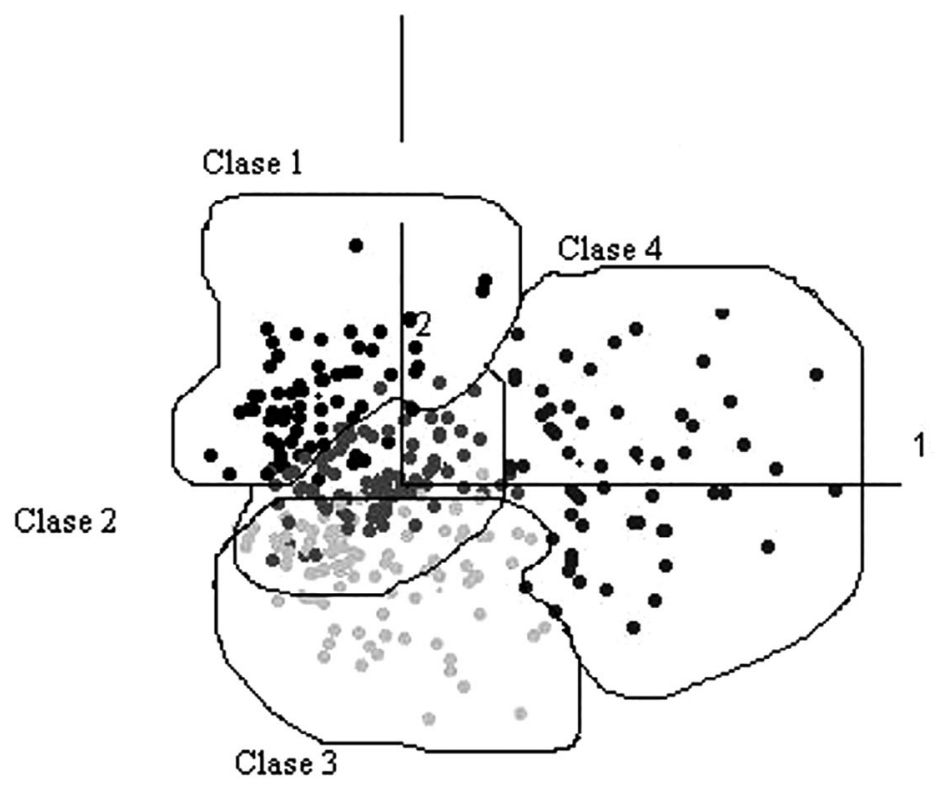

El número medio de supuestos respondidos adecuadamente es mayor en las primeras clases, teniendo la clase uno la media más elevada con 5 supuestos y la tercera clase la más baja con 2,5. Existen diferencias significativas en estas medias entre las clases $(F(3,251)=39,84 p \leq .001)$, de modo que la clase tres y cuatro se diferencian de todas las restantes, estableciéndose tres subconjuntos, uno formado por la clase uno y dos, otro formado por la clase tres y otro por la cuatro. Además, la primera clase se asocia significativamente con el nivel elevado de respuestas correctas (7 o más supuestos adecuados), la segunda clase con el nivel medio y la tercera con el nivel bajo. La cuarta clase tiene su mayor porcentaje en el nivel bajo, aunque no se asocia estadísticamente.

Responder adecuadamente a los supuestos se asocia significativamente con pertenecer a la primera clase, a excepción del segundo, cuarto y quinto donde las respuestas adecuadas se asocian con pertenecer a la segunda clase. La clase tercera se asocia con las respuestas inadecuadas en todos los supuestos a excepción del segundo. Cabe mencionar que el supuesto nueve no muestra asociación estadísticamente significativa con ninguna clase. 
Posteriormente se han analizado las clases en relación con las variables claves como CI, SI y aspectos de intervención. Las medias más elevadas de los aspectos de intervención se dan en la clase primera, a excepción de ser consciente de los propios sesgos que se ubica en la segunda clase. Las medias más bajas en su mayoría se presentan en la cuarta clase.

En relación con la CI, la mayor media se sitúa en la clase dos (considerando desde 1 nivel de incompetencia a 4 nivel competencia) y la menor en la clase cuatro. Se producen asociaciones entre los niveles de competencia de las dimensiones de conocimiento y habilidades con la primera clase y de conciencia, motivación, encuentro y del total de CI con la segunda. Los niveles de incompetencia de todas las dimensiones y del total de CI se asocian con la cuarta clase.

Si tomamos la SI tanto de manera conjunta como en cada una de sus dimensiones, las mayores medias se ubican en la primera clase y las menores (que se sitúan en nivel medio de la escala) en la cuarta clase. Se establecen asociaciones significativas entre los niveles medios de todas ellas y del total de la escala con la cuarta clase y de los niveles altos de confianza, disfrute y del total de SI con la primera y de la dimensión de implicación con la segunda clase.

Tabla 6. Asociaciones entre clases y respuestas a los supuestos prácticos

\begin{tabular}{|c|c|c|c|c|}
\hline & $\begin{array}{c}\text { Asociación } \\
\text { con responder } \\
\text { adecuadamente }\end{array}$ & $\begin{array}{c}\text { Asociación } \\
\text { con responder } \\
\text { inadecuadamente }\end{array}$ & Significancia estadística & $\begin{array}{l}\text { Coeficiente de } \\
\text { contingencia }\end{array}$ \\
\hline Supuesto 1 & Clase 1 & Clase 3 & $\chi^{2}(3, \mathrm{~N}=230)=17,54 \mathrm{p}=.001$ & 0,266 \\
\hline Supuesto 2 & Clase 2 & Clase 4 & $\chi 2(3, \mathrm{~N}=197)=22,21 \mathrm{p} \leq .001$ & 0,318 \\
\hline Supuesto 3 & Clase 1 & Clase 3 & $\chi^{2}(3, \mathrm{~N}=230)=16,70 \mathrm{p}=.001$ & 0,260 \\
\hline Supuesto 4 & Clase 2 & & $\chi 2(3, N=192)=15,07 \mathrm{p}=.002$ & 0,270 \\
\hline Supuesto 5 & & & $\chi 2(3, \mathrm{~N}=218)=30,52 \mathrm{p} \leq .001$ & 0,350 \\
\hline Supuesto 6 & Clase 1 & & $\chi 2(3, N=213)=20,05 p \leq .001$ & 0,293 \\
\hline Supuesto 7 & & & $\chi 2(3, N=211)=24,55 \mathrm{p} \leq .001$ & 0,323 \\
\hline Supuesto 8 & & Clase 3 y Clase 4 & $\chi 2(3, \mathrm{~N}=197)=24,60 \mathrm{p} \leq .001$ & 0,333 \\
\hline Supuesto 9 & \multirow{2}{*}{\multicolumn{2}{|c|}{ No asoc. }} & No asociación significativa & \\
\hline $\begin{array}{c}\text { TOTAL } \\
\text { SUPUESTOS }\end{array}$ & & & $\chi 2(6, N=253)=118,07 \mathrm{p} \leq .001$ & 0,565 \\
\hline
\end{tabular}




\begin{tabular}{|c|c|c|c|c|}
\hline $\mathrm{CI}$ & Nivel Incompetencia & Nivel Competencia & & \\
\hline Conciencia & & Clase 2 & $\chi 2(3, \mathrm{~N}=250)=15,40 \mathrm{p}=.001$ & 0,241 \\
\hline Motivación & & & $\chi 2(3, \mathrm{~N}=253)=51,08 \mathrm{p} \leq .001$ & 0,410 \\
\hline Conocimiento & & Clase 1 & $\chi 2(3, \mathrm{~N}=252)=101,6 \mathrm{p} \leq .001$ & 0,536 \\
\hline Habilidades & Clase 4 & & $\chi 2(3, \mathrm{~N}=250)=81,80 \mathrm{p} \leq .001$ & 0,505 \\
\hline Encuentro & & Clase 2 & $\chi 2(3, \mathrm{~N}=251)=63,67 \mathrm{p} \leq .001$ & 0,450 \\
\hline TOTAL CI & & & $\chi 2(3, N=246)=110,25 \mathrm{p} \leq .001$ & 0,556 \\
\hline SI & Media & Elevada & & \\
\hline Implicación & & Clase 2 & $\chi 2(3, \mathrm{~N}=180)=17,41 \mathrm{p}=.001$ & 0,297 \\
\hline Respeto & & & $\chi 2(6, N=214)=13,19 \mathrm{p}=.040$ & 0,241 \\
\hline Confianza & & Clase 1 & $\chi 2(6, N=225)=52,44 \mathrm{p} \leq .001$ & 0,434 \\
\hline Disfrute & Clase 4 & & $\chi 2(6, \mathrm{~N}=232)=23,28 \mathrm{p}=.001$ & 0,302 \\
\hline Atención & & & $\chi 2(6, N=215)=47,25 \mathrm{p} \leq .001$ & 0,424 \\
\hline TOTAL SI & & Clase 1 & $\chi 2(6, \mathrm{~N}=249)=85,92 \mathrm{p} \leq .001$ & 0,506 \\
\hline
\end{tabular}

Tabla 7. Descriptivos por variables claves de análisis

\begin{tabular}{|c|c|c|c|c|c|}
\hline & & $\begin{array}{l}\text { Clase } 1 \\
(\mathrm{~N}=52)\end{array}$ & $\begin{array}{l}\text { Clase } 2 \\
(\mathrm{~N}=73)\end{array}$ & $\begin{array}{l}\text { Clase } 3 \\
(\mathrm{~N}=81)\end{array}$ & $\begin{array}{l}\text { Clase } 4 \\
(\mathrm{~N}=47)\end{array}$ \\
\hline \multirow{3}{*}{$\begin{array}{c}\text { Total } \\
\text { supuestos (\%) }\end{array}$} & Baja & 13,5 & 13,7 & 86,4 & 52,2 \\
\hline & Media & 61,5 & 76,7 & 12,3 & 43,5 \\
\hline & Alta & 25,0 & 9,6 & 1,2 & 4,3 \\
\hline \multicolumn{2}{|c|}{$\begin{array}{c}\text { Media supuestos adecuados } \\
(1-9)\end{array}$} & $5,13(\mathrm{SD}=1,80)$ & $4,78(\mathrm{SD}=1,520)$ & $2,52(\mathrm{SD}=1,361)$ & $3,63(\mathrm{SD}=1,691)$ \\
\hline \multirow{6}{*}{$\begin{array}{c}\text { Escala } \\
\text { Intervención } \\
\text { (1-5 grado } \\
\text { acuerdo) }\end{array}$} & $\begin{array}{l}\text { Soy consciente } \\
\text { de mis sesgos }\end{array}$ & $2,00(\mathrm{SD}=0,950)$ & $2,33(\mathrm{SD}=0,852)$ & $2,00(\mathrm{SD}=0,920)$ & $2,07(\mathrm{SD}=0,848)$ \\
\hline & Habilidades & $2,96(\mathrm{SD}=0,196)$ & $2,50(\mathrm{SD}=0,654)$ & $2,51(\mathrm{SD}=0,636)$ & $2,04(\mathrm{SD}=0,796)$ \\
\hline & $\begin{array}{l}\text { Percibir la } \\
\text { diversidad }\end{array}$ & $2,92(\mathrm{SD}=0,272)$ & $2,23(\mathrm{SD}=0,685)$ & $2,29(\mathrm{SD}=0,715)$ & $1,80(\mathrm{SD}=0,734)$ \\
\hline & $\begin{array}{l}\text { Diseñar un } \\
\text { proyecto }\end{array}$ & $2,63(\mathrm{SD}=0,564)$ & $2,01(\mathrm{SD}=0,752)$ & $2,16(\mathrm{SD}=0,829)$ & $1,40(\mathrm{SD}=0,623)$ \\
\hline & $\begin{array}{l}\text { Conocimientos } \\
\text { intervención }\end{array}$ & $2,86(\mathrm{SD}=0,401)$ & $2,09(\mathrm{SD}=0,737)$ & $2,30(\mathrm{SD}=0,660)$ & $1,53(\mathrm{SD}=0,726)$ \\
\hline & Total escala & $3,82(\mathrm{SD}=0,545)$ & $3,26(\mathrm{SD}=0525)$ & $3,31(\mathrm{SD}=0,634)$ & $2,69(\mathrm{SD}=0,623)$ \\
\hline \multirow{6}{*}{$\begin{array}{c}\text { Competencia } \\
\text { Intercultural } \\
\quad(1-4)\end{array}$} & Conciencia & $2,30(\mathrm{SD}=1,015)$ & $2,71(\mathrm{SD}=0,874)$ & $2,26(\mathrm{SD}=1,010)$ & $1,96(\mathrm{SD}=0,868)$ \\
\hline & Motivación & $2,02(\mathrm{SD}=1,379)$ & $2,74(\mathrm{SD}=1,214)$ & $3,19(\mathrm{SD}=1,074)$ & $1,85(\mathrm{SD}=0,908)$ \\
\hline & Conocimiento & $3,06(\mathrm{SD}=0,366)$ & $2,86(\mathrm{SD}=0,484)$ & $2,83(\mathrm{SD}=0,495)$ & $2,04(\mathrm{SD}=0,415)$ \\
\hline & Habilidades & $3,24(\mathrm{SD}=0,555)$ & $3,07(\mathrm{SD}=0,561)$ & $3,08(\mathrm{SD}=0,652)$ & $2,21(\mathrm{SD}=0,690)$ \\
\hline & Encuentro & $3,18(\mathrm{SD}=0,800)$ & $3,03(\mathrm{SD}=0,707)$ & $3,00(\mathrm{SD}=0,822)$ & $2,11(\mathrm{SD}=0,759)$ \\
\hline & Total CI & $13,96(\mathrm{SD}=2,259)$ & $14,42(\mathrm{SD}=1,750)$ & $14,33(\mathrm{SD}=1,868)$ & $10,13(S D=2,146)$ \\
\hline
\end{tabular}




\begin{tabular}{cccccc}
\hline & Implicación & $4,38(\mathrm{SD}=0,514)$ & $4,20(\mathrm{SD}=0,506)$ & $4,15(\mathrm{SD}=0,461)$ & $3,72(\mathrm{SD}=0,611)$ \\
Sensibilidad & Respeto & $4,59(\mathrm{SD}=0,613)$ & $4,54(\mathrm{SD}=0,391)$ & $4,58(\mathrm{SD}=0,386)$ & $4,12(\mathrm{SD}=0,779)$ \\
$\begin{array}{c}\text { Intercultural } \\
(1-5)\end{array}$ & Confianza & $4,08(\mathrm{SD}=0,505)$ & $3,56(\mathrm{SD}=0,574)$ & $3,74(\mathrm{SD}=0,536)$ & $3,20(\mathrm{SD}=0,645)$ \\
& Disfrute & $4,37(\mathrm{SD}=0,864)$ & $4,14(\mathrm{SD}=0,841)$ & $4,14(\mathrm{SD}=0,824)$ & $3,57(\mathrm{SD}=0,885)$ \\
& Atención & $4,16(\mathrm{SD}=0,778)$ & $4,16(\mathrm{SD}=0,606)$ & $4,06(\mathrm{SD}=0,514)$ & $3,27(\mathrm{SD}=0,553)$ \\
& Total escala & $4,32(\mathrm{SD}=0,534)$ & $4,14(\mathrm{SD}=0,329)$ & $4,17(\mathrm{SD}=0,293)$ & $3,69(\mathrm{SD}=0,417)$ \\
\hline
\end{tabular}

\section{Discusión y conclusiones}

Los resultados ponen de manifiesto algunas asociaciones significativas entre las respuestas a los supuestos planteados y las variables claves del estudio.

En relación a la caracterización no se producen diferencias en el número de supuestos respondidos adecuadamente debido a pertenecer a una u otra profesión de las que conforman los equipos base de los Servicios Sociales Comunitarios. La inexistencia de asociaciones representativas con ciertas variables sociodemográficas pone de manifiesto que las características personales/ profesionales no influyen significativamente en las respuestas.

Sin embargo, el hecho de ser un profesional de mayor edad (entre los $50 \mathrm{y}$ 64 años) se relaciona con responder inadecuadamente a los supuestos cuarto y quinto, reflejando menor flexibilidad cognitiva, empatía y menor comprensión de aspectos de otras culturas diferentes a la propia. Esto puede estar relacionado con un menor acceso durante su trayectoria profesional a cuestiones formativas y de sensibilización con respecto a la diversidad cultural, entre otros aspectos.

Hay otras cuestiones de caracterización personal que destacan como tener formación en diversidad y mantener contacto con personas de origen extranjero, ya que ambas se asocian con responder adecuadamente al supuesto sexto relacionado con mayor habilidad para formar y mantener relaciones y con el mantenimiento de la distancia social adecuada, algo que sin duda se favorece cuando el profesional dispone de formación específica sobre la diversidad cultural y cuando además mantiene contacto con personas de culturas diferentes a la propia, propiciando la familiarización y el aprendizaje en el manejo de las relaciones sociales, aspectos vinculados con la primera de las clases extraídas. El contacto también propicia respuestas adecuadas al supuesto séptimo (en el que el profesional insiste en los derechos al usuario), que muestra las habilidades de conocimiento y comprensión tanto de la propia cultura como de otras culturas diferentes, que de nuevo se asocia con pertenecer a la primera clase.

Poseer un cierto nivel de CI se asocia, en ciertos casos, con resolver con acierto las intervenciones profesionales. Cabe destacar que son los supuestos segundo y octavo los que tienen asociaciones en este sentido. Concretamente el segundo supuesto que refleja el conocer y comprender tanto la propia cultura 
como otras, se relaciona con niveles de CI elevados, sobre todo de conciencia y de conocimiento, así como con una elevada implicación de la SI y con pertenecer a la segunda de las cuatro clases extraídas.

El no saber mantener la distancia social apropiada (supuesto octavo) está asociado con una menor disponibilidad al encuentro intercultural, así como menor habilidad de gestión de la diversidad en la intervención profesional. Aquellos profesionales que sí disponen de buenas respuestas a esta supuesto, y por ello se ubican en la clase primera, con niveles elevados de competencia en el encuentro y en las habilidades mencionadas.

El sexto supuesto también refleja la competencia de saber manejar adecuadamente la distancia social en las interacciones y se relaciona con disponer de formación en diversidad y con mantener contacto con personas extranjeras, variables claves para el desarrollo de la competencia intercultural.

Responder adecuadamente a mayor número de supuestos se asocia con aquellas personas que tienen mayor conciencia intercultural, mayor motivación por el encuentro. Esta mayor motivación también conlleva una adecuada respuesta al supuesto primero donde se refleja la habilidad para no enjuiciar siendo consciente de las diferencias culturales existentes. Es la clase primera la que aglutina el mayor número de supuestos adecuadamente resueltos, que se asocia a una mayor conciencia de los propios sesgos y prejuicios posibles hacia las diferencias culturales (como aspecto de intervención).

Las clases extraídas han puesto de manifiesto la existencia de aspectos claves para disponer de respuestas adecuadas a los supuestos y que se relacionan con niveles de $\mathrm{CI}$ elevados en las diferentes dimensiones contempladas, así como niveles elevados de SI que permiten disponer de aspectos claves para el desarrollo de la praxis profesional en contextos de diversidad. Junto a estas cuestiones también aparece asociado el hecho de tener contacto con personas extranjeras y formación en diversidad o conocimiento de idiomas. La neutralidad, correspondida por la clase tercera de la tipología, se ubica en niveles bajos de respuestas adecuadas a los supuestos y una indiferencia en cuestiones de intervención fundamentalmente.

Las competencias asociadas a estos supuestos son bastante relevantes en el desempeño profesional en contextos de diversidad cultural. El hecho de que la formación específica y el contacto con personas de culturas diferentes sea relevante en la asociación con responder de un modo adecuado a ciertos supuestos, pone de manifiesto que se trata de cuestiones claves a considerar en estas profesiones y en el propio reciclaje y formación continua de los profesionales de los Servicios Sociales Comunitarios. Esta formación y el contacto son elementos diferenciadores en la práctica y en cuestiones que tienen que 
ver con la interacción directa con el otro (motivación y habilidades). Trabajar la formación en diversidad e interculturalidad de manera transversal permitirá aumentar conocimientos, habilidades, actitudes que, unido a lo positivo que proporciona el contacto con personas extranjeras, permitirá un mejor y más adecuado desarrollo profesional en contextos de diversidad cultural.

Resulta por lo tanto más relevante, y al mismo tiempo se desarrollan de un modo más adecuado, cuestiones que tienen que ver con el conocimiento y la comprensión de la propia cultura y de aspectos de otras culturas, así como la habilidad de no enjuiciar, saber tener relaciones con personas de diversas culturas y, al mismo tiempo, mantener las distancia social adecuada en cada momento (y que son los supuestos que más asociaciones y relevancia han adquirido en los resultados obtenidos). Estos aspectos están relacionados con competencias y habilidades interculturales que tienen los profesionales de los Servicios Sociales Comunitarios como la conciencia intercultural, la motivación por el encuentro y el propio desarrollo de las interacciones. De igual modo, destaca la implicación de la SI en la gestión y la comprensión de las diferencias y características de personas y situaciones de otras culturas diferentes a la propia, manejando y siendo conscientes de los propios sesgos personales.

En este sentido, y siguiendo las recomendaciones de la American Psychological Association (2003), se puede trabajar en cuanto a los profesionales de la intervención social el mantenimiento y mejora del conocimiento de las propias creencias, valores, actitudes, realizando un esfuerzo por el cambio de las percepciones automáticas positivas para el endogrupo y negativas para el exogrupo, pudiendo cambiar sesgos y prejuicios. Habría que trabajar cuestiones que están menos desarrolladas y/o afianzadas como la tolerancia a la ambigüedad en situaciones profesionales complejas donde está presente la diversidad cultural, reforzar la flexibilidad cognitiva, la empatía y la habilidad de resolución de conflictos en estas situaciones y con personas usuarias de diferentes culturas.

Estas cuestiones pueden y deben ser trabajadas con los profesionales de los Servicios Sociales Comunitarios, con todas las profesiones presentes dado que no se han hallado diferencias relevantes en función de la profesión. Se pueden elaborar propuestas de formación encaminadas a mejorar y adecuar los servicios y la propia CI profesional.

Pero además de realizar este trabajo de formación y sensibilización profesional continua, habría que incidir en las etapas de formación universitaria. Las Facultades de las diferentes profesiones deben potenciar el desarrollo de competencias interculturales específicas y transversales. El ámbito universitario debe propiciar la reflexión y la reformulación de la definición de CI, reconociendo su carácter complejo, dinámico, interseccional los elementos de poder 
y las macro estructuras implicadas además de los elementos individuales y comunitarios culturales presentes en las situaciones de atención e intervención profesional.

\section{Bibliografía}

American Psychological Association (2010). Ethical Principles of Psychologists and Code of Conduct. United States: American Psychological Association.

Asociación Estatal de Educación Social (2004). Código deontológico del educador y la educadora social. Barcelona, España: Asociación Estatal de Educación Social.

BalCaZAR, F. FABricio, SuARez-BAlCAZAR, M. y Willis, E. (2010). Cultural competence: A review of conceptual frameworks. En Balcazar, Suarez-Balcazar, Taylor-Ritzler and Keys (Eds.), Race, culture, and disability: Rehabilitation science and practice (pp.281-305). Boston, EEUU: Jones \& Bartlett.

Berry, J. V., Pootinga, Y., Segall, M. y Dasen, P. (2002). Cross-cultural Psychology. Research and applications. New York, EEUU: Cambridge University Press.

COHEN, J. (1988). Statistical power analysis for the behavioral sciences. Nueva York, EEUU: Academic Press. https://doi.org/10.1002/bs.3830330104

Consejo General del Trabajo Social (2012). El Código Deontológico del Trabajo Social. Madrid, España: Consejo General del Trabajo Social.

DANA, R. AND ALLEN, J. (2008). Cultural competency training in a global society. New York, EEUU: Springer. https://doi.org/10.1007/978-0-387-79822-6

DomeneCh-Rodríguez, M. M., Baumann, A. y SChwartZ, A. (2011). Cultural Adaptation of an Evidence Based Intervention: From Theory to Practice in a Latino/a Community Context. American Journal Community Psychology, 47, 170-186. https://doi.org/10.1007/s10464-010-9371-4

Dumas, J. E., Rollock, D., Prinz, R., Hops, H. y Blechman, E. (1999). Cultural sensitivity: Problems and solutions in applied and preventive intervention. Applied \& Preventive Psychology, 8, 175-196. https://doi.org/10.1016/ S0962-1849(05)80076-9

GARRAN, A. M. y WeRKMEISTER, L. (2013). Cultural Competence Revisited. Journal of Ethnic \& Cultural Diversity in Social Work, 22 (97), 111-121. https://doi.org /10.1080/15313204.2013.785337

GENG, C. y AwA, N. (1992). Measuring intercultural effectiveness: An integrative approach. International Journal of Intercultural Relations, 16, 311-328. https:// doi.org/10.1016/0147-1767(92)90055-Y

Guo-Ming, C. y Starosta, W. (2000). The development and validation of the intercultural sensitivity scale. Human Communication, 3, 1-15.

instituto Nacional de Estadística de España (2013). Población por lugar de nacimiento. Madrid, España: INE. Gobierno de España. 
INTERNATIONAL FEDERATION OF SOCIAL WORKERS (2004). Statement of ethical principles. Recuperado de http://ifsw.org/policies/statement-of-ethical-principles/

INTERNATIONAL ASSOCIATION OF SCHOOLS OF SOCIAL WORK (2004). Ethics in social work, statement of principles. Australia: Adelaide. Recuperado de http://www. ifsw.org/f38000032.html

Julve, M. y PAlOMO, B. (2005). La competencia comunicativa intercultural en la prestación de servicios. Glosas didácticas-Revista electrónica internacional, 15, 26-38.

Kohli, H. K., Huber, R. y FAUL, A. (2010). Historical and Theoretical Development of Culturally Competent Social Work Practice. Journal of Teaching in Social Work, 30 (3), 252-271. https://doi.org/10.1080/08841233.2010.499091

Martínez, M.F., Martínez, J. y CAlzado, V. (2006). La Competencia Cultural como referente de la Diversidad Humana en la Prestación de Servicios y la Intervención Social. Intervención Psicosocial, 15(3), 331-350. https://doi. org/10.4321/S1132-05592006000300007

MAYA, I. (2002). Estrategias de entrenamiento de las habilidades de comunicación intercultural. Portularia, 2, 91-108.

NATIONAL ASSOCIATION OF SOCIAL WORKERS (2001). Standards for cultural competence in social work practice. Recuperado de http://www.naswdc.org/ practice/ standards/NAswculturalstandards.pdf

Skelton, T. y Allen, T. (1999). Culture and global change. London, UK: Routledge.

Ungar, M. (2002). Deeper, More Social Ecological Social Work Practice. Social Service Review, 76(3), 480-497. https://doi.org/10.1086/341185

VÁzquez-Aguado, O., Fernández, M., y FernándeZ-Borrero, M.A. (2010). Good intercultural practices in social services projects. Journal of Social Service Research, 36(4), 303-320. https://doi.org/10.1080/01488376.2010.493853

VÁzquez-Aguado, O., Fernández-Borrero, M.A., y Álvarez-PÉrez, P. (2014). La aportación de los grados al desarrollo de la sensibilidad y la competencia intercultural. Perspectiva comparada entre Trabajo Social y Psicología. Cuadernos de Trabajo Social, 27(2), 307-317. https://doi.org/10.5209/rev_CUTS.2014. v27.n2.44679

VILÁ, R. (2005). La Competencia Comunicativa Intercultural. Un estudio en el Primer Ciclo de la E.S.O. Tesis Doctoral. Barcelona, España: Universidad de Barcelona.

Wiseman, R. L., HAMmer, M. y NishidA, H. (1989). Predictors of intercultural communication competence. International Journal of Intercultural Relations, 13, 349-370. https://doi.org/10.1016/0147-1767(89)90017-5

\section{ANTECEDENTES DE DIFUSIÓN}

La difusión de los resultados del presente estudio ha contado con el apoyo del Ministerio de Educación, Cultura y Deporte del Gobierno de España a través del Programa Nacional de Formación de Profesorado Universitario (FPU15/03909) de la tercera autora. 
Cita bibliográfica: Campos Vidal, J. F., Cardona Cardona, J. y Cuartero Castañer, M. E. (2017). Afrontar el desgaste: cuidado y mecanismos paliativos de la fatiga por compasión [Facing the wear: care and palliative mechanisms of compassion fatigue]. Alternativas. Cuadernos de Trabajo Social, 24, 119-136. https://doi.org/10.14198/ALTERN2017.24.07

\title{
AFRONTAR EL DESGASTE: CUIDADO Y MECANISMOS PALIATIVOS DE LA FATIGA POR COMPASIÓN* FACING THE WEAR: CARE AND PALLIATIVE MECHANISMS OF COMPASSION FATIGUE
}

\author{
José FRANCISCO CAMPOS-VIDAL** iD \\ Departamento de Filosofía y Trabajo Social, Universidad de las Islas Baleares, España \\ quico.campos@uib.es \\ JOSEFA CARDONA-CARDONA \\ Departamento de Filosofía y Trabajo Social, Universidad de las Islas Baleares, España \\ jp.cardona@uib.es \\ María Elena CuARTERo-Castañer iD \\ Departamento de Filosofía y Trabajo Social, Universidad de las Islas Baleares, España \\ me.cuartero@uib.es
}

\begin{abstract}
Resumen
El concepto de Fatiga por Compasión (Compassion Fatigue) o Desgaste por Empatía es común en la literatura científica de Trabajo Social internacional, aunque sorprendentemente inexistente en la literatura española. Puede definirse la fatiga por compasión como estado de agotamiento y disfunción biológica, psicológica y relacional, resultado de la exposición inmediata o prolongada al estrés por compasión y como el resultado final del proceso progresivo y acumulativo consecuencia del contacto prolongado, continuado e intenso con clientes o pacientes, el uso de uno mismo y la exposición al estrés.

El mantenimiento del desgaste puede derivar en cuadros severos típicos de traumatización secundaria o en el inicio del Síndrome del Profesional Quemado (Burnout). En todo caso, si un profesional del trabajo
\end{abstract}

social experimenta dicho fenómeno, su capacidad para empatizar, conectar y ayudar a sus clientes se ve gravemente disminuida. Paradójicamente, la fatiga por compasión es por una parte, necesaria e inevitable si se desarrolla una buena práctica; por otra, peligrosa e inhabilitante si no se compensa mediante otros mecanismos.

La fatiga por compasión no se puede prevenirse pero puede y debe paliarse de forma consciente. Incrementar la capacidad de reconocer y minimizar el impacto del desgaste es una responsabilidad del profesional y de la organización en la cual trabaja. Por último, el trabajo plantea el qué hacer ante este fenómeno y de qué manera cuidarse personal, profesional e institucionalmente.

Palabras clave: Trabajo Social; Fatiga por compasión; desgaste profesional; relación de ayuda; autocuidado.

* Parte de este trabajo fue presentado en forma de ponencia en XIIICongreso Estatal y I Iberoamericano de Trabajo Social, que se ha celebrado en Mérida (Badajoz) del 19 al 21 de octubre de 2017.

** Universidad de las Islas Baleares, Departamento de Filosofía y Trabajo Social. Ctra. de Valldemossa, s/n. Ed. Beatriu de Pinós, D3, 07122 Palma de Mallorca, España. E-mail: quico.campos@uib.es 


\begin{abstract}
The Compassion Fatigue concept is common in the scientific literature of International Social Work, although surprisingly nonexistent in Spanish literature. Fatigue can be defined by compassion as a state of exhaustion and biological, psychological and relational dysfunction, the result of immediate or prolonged exposure to compassionate stress and as the final result of the progressive and cumulative process resulting from prolonged, continuous and intense contact with clients or clients. patients, the use of oneself and exposure to stress.

The maintenance of the wear can lead to severe pictures typical of secondary traumatization or the onset of Burned Professional Syndrome (Burnout). In any case, if a social work professional experiences this phenomenon,
\end{abstract}

\section{Extended abstract}

Summary. The concept of Compassion Fatigue is common in the scientific literature of International Social Work, although surprisingly nonexistent in the literature of our country. Compassion fatigue can be defined as a state of exhaustion and biological, psychological and relational dysfunction, the result of immediate or prolonged exposure to compassion stress and as the final result of the progressive and cumulative process resulting from prolonged, continuous and intense contact with clients, patients, the use of oneself and exposure to stress.

The maintenance of the wear can lead to severe typical pictures of secondary traumatization or the beginning of Burned Professional Syndrome (Burnout). In any case, if a social work professional experiences this phenomenon, their ability to empathize, connect and help their clients is severely diminished. Paradoxically, the compassion fatigue is on the one hand, necessary and inevitable if good practice is developed; on the other, dangerous and disabling if it is not compensated by other mechanisms. Compassion fatigue can not be prevented but it can and must be palliated consciously. Increasing the ability to recognize and minimize the impact of attrition is a responsibility of the professional and the organization in which he works.

The investigation of the processes and mechanisms of professional burnout that have developed in the practice of social work in Spain, have revolved almost exclusively on the phenomenon of Burnout Syndrome (Lázaro Fernández, 2004, Blanco Montilla, 2006, Facal Fondo, 2012, De la Fuente Roldán \& Sánchez Moreno 2012, Barrera Algarín, Malagón Bernal \& Sarasola Sánchez-Serrano, 2015).

The excessive breadth and scope of this idea of attrition, the inaccuracy and confusion existing in the scientific literature about it (Campos, Cardona, Cuartero \& Bolaños, 2015). and the profusion of the their ability to empathize, connect and help their clients is severely diminished. Paradoxically, compassion fatigue is, on the one hand, necessary and inevitable if good practice is developed; on the other, dangerous and disabling if it is not compensated by other mechanisms. Compassion fatigue can not be prevented but it can and must be palliated consciously. Increasing the ability to recognize and minimize the impact of attrition is a responsibility of the professional and the organization in which he works. Finally, the work raises what to do about this phenomenon and how to take care of yourself, professionally and institutionally.

Keywords: Social work; compassion fatigue; professional wear; help relationship; self-care

concept based on the dissemination of Maslach's works (1986) it has facilitated the extension of a single perception of the phenomenon of professional burnout in the set of professions that, like social work, focused on the context of aid relationships. The clinical practice and the empirical evidence have shown the fact that this broad and generic view of professional burnout is not the only possible nor, in certain contexts of professional activity, the most appropriate. Based on the evidence that wear occurs in the practice of social work professionals (Figley, 1995, Adams, Boscarino \& Figley, 2006, Bourassa, 2009, Dekel \& Baum, 2010 y Campos $\&$ Cardona, 2017). We consider that it is necessary to provide theoretical perspectives and alternative practices to the dominant theses about wear.

Compassion fatigue has been defined as «the state of biological, psychological and social exhaustion and dysfunction, the result of prolonged exposure to compassion stress and all that it evokes» (Lynch \& Lobo, 2012: 21-25). For Figley (2002: 14-30), «Compassion fatigue like any other form of fatigue reduces our capacity or our interest in supporting the suffering of others». If a professional experiences this phenomenon, their ability to empathize, connect and help their clients is severely diminished. Figley (2014) considers compassion fatigue as the natural emotional behaviors and reactions derived from knowing a traumatic event experienced by a significant person, that is, an indirect traumatization of rapid and sudden onset as a consequence of helping those who experience the trauma. directly and/or primary.

The latest published works abandon the restricted vision provided by Psychotraumatology and extend the concept of compassion fatigue, understanding it as the progressive and accumulated result of intense contact with people who suffer and by exposure to stress and that include all professionals who they develop a helping relationship (Adams, Boscarino \& Figley, 2006, Figley \& Bride, 2007, Figley, Adams \& Boscarino, 2008, Dekel \& Baum, 2010). The common 
denominator of all the proposals related to its definition rests on the concept of empathy, not on the concept of trauma.

Associated with the "costs of care» to other people who suffer, numerous terms used have made possible a certain theoretical-conceptual confusion (Acinas, 2011). As Figley (Figley, 1995, 2002, Figley \& Bride, 2007, Figley, 2014) has already pointed out, various terms have been used to refer to the wear that describes the current meaning of compassion fatigue. We refer to the concepts of Secondary Traumatic Stress (traumatic stress derived from contact with the victim or the traumatic event), Post-Traumatic Stress, Vicarious Traumatization, Countertransference and also the wellknown Burnout Syndrome (Lynch \& Lobo, 2012), as well as emotional contagion (Fernández-Pinto, LópezPérez \& Márquez, 2008).

At times, compassion fatigue has been considered a sub-typology of Burnout Syndrome. Several authors (Najjar, Davis, Beck-Coon \& Carney-Doebbeling, 2009) consider that compassion fatigue is a unique form of Burnout, in which the cause of the discomfort is not so related to the stressors of work as with the empathic response that professionals who work with empathy provide. Sabo $(2006,2011)$ describes the Burnout Syndrome as a gradual process resulting from the responses we give in our work environment, while compassion fatigue has a sharp onset that results from the attention to the people who are suffering, product of the experience of taking charge, empathically, of the other's discomfort, being only present in the professions related to helping relationships (Jacobson, Rothschild, Mirza \& Shapiro, 2013, Stamm, 2002). Compassion fatigue is the natural, predictable, treatable, and paliable consequence of working with people who suffer; it is the emotional residue resulting from exposure to work with those who suffer the consequences of traumatic events (Acinas, 2012, Rothschild, 2006).

As a phenomenon inherent to the help process, compassion fatigue is not preventable. Its existence and manifestation reflects the development of a good relational link and an adequate use of our empathic capacity. However, this does not mean that we can not develop conscious actions to minimize their effects. Bride, Radey \& Figley (2007) point out that the prevalence of compassion fatigue is the result, always unbalanced, of the balance between compassion satisfaction and compassion fatigue. Satisfaction for compassion is related to the subjective perception of a job well done, the emotionally significant connection with our clients and the satisfaction of the psychoemotional needs of the professional. This balance, of leaning dangerously towards a significant increase of the compassion fatigue, increases the risk of emotional disconnection, the danger of making wrong judgments or of organizing poorly and the process of change.

From this perspective, the cost of caring must be compensated with self-care. We can understand selfcare as «those activities carried out by individuals, families or communities, with the purpose of promoting health, preventing disease, limiting it when it exists or re-establishing it when necessary.» (Ginés \& Carvalho, 2010:307), having an institutional, personal or community dimension. If this compensation is not given, compassion fatigue will prevail compassion satisfaction. Bride, Radey \& Figley (2007) propose that alleviating the compassion fatigue, say, counterbalance the structural imbalance that will always exist between satisfaction and fatigue, means developing four areas (Campos \& Cardona, 2017).

a) Development of positive relationships with clients, that is, permanently activate the empathic response and the dimensions of the aid alliance. It is, in short, to work well, consciously well. They also aim to cover the needs of the professional such as satisfaction with the relationship provided or the recognition of colleagues. b) Non-specific self-care related to the maintenance of recognized standards of self-care that define the lifestyle (rest, sleep and eat well, maintain relationships and networks, hobbies, etc.). It is necessary to monitor frequently the fulfillment of the (conscious) plan of self-care and to update it periodically.

c) Intentional self-care linked to the permanent review of professional practice and its impact on itself, resorting to supervision, review of practice, participation in professional forums, study, biophysical balance (food, exercise, rest and pleasure). d) Stress management strategies through the care and development of hobbies, relaxation, yoga, mindfulness, artistic expressions, therapeutic writing, etc.

Keywords: Social work; fatigue for compassion professional wear; help relationship; self-care 


\section{Introducción}

La investigación de los procesos y mecanismos de desgaste profesional que se han desarrollado en el marco de referencia del Trabajo Social en España, han girado casi exclusivamente sobre el fenómeno del Síndrome de Burnout, (Lázaro Fernández, 2004, Blanco Montilla, 2006, Facal Fondo, 2012, De la Fuente Roldán y Sánchez Moreno, 2012, Barrera Algarín, Malagón Bernal y Sarasola Sánchez-Serrano, 2015).

La bi-direccionalidad que Feundenberger (1974) planteó a la hora de describir y definir conceptualmente el Síndrome de Burnout, ha sido una de las fuentes de confusión más significativas a la hora de aprehender el concepto, investigar el fenómeno y definir su alcance. El autor describe el síndrome como la respuesta al estrés laboral crónico, que se pone en evidencia por la actitud y sentimientos negativos dirigidos hacia las personas con las cuales se trabaja, hacia el propio rol profesional y a la percepción de agotamiento emocional. Feundenberger (1974) indica que este tipo de fenómeno es observable en el conjunto de trabajadores que están en contacto directo con personas y, más tarde, amplia esta perspectiva hacia cualquier tipo de profesional. La excesiva amplitud y alcance de esta idea de desgaste, la inexactitud y confusión existente en la literatura científica al respecto (Campos, Cardona, Cuartero, y Bolaños, 2015) y la profusión del concepto a partir de la divulgación de los trabajos de Maslach (1986) ha facilitado la extensión de una única percepción del fenómeno del desgaste profesional en el conjunto de profesiones que, como el Trabajo Social, se centraban en el contexto de las relaciones de ayuda.

La práctica clínica y la evidencia empírica han puesto de manifiesto el hecho de que esta visión tan amplia y genérica del desgaste profesional, ni es la única posible ni, en determinados contextos de actividad profesional, la más adecuada. Sin embargo, ha sido durante décadas la idea hegemónica que ha guiado la investigación sobre el impacto del desgaste profesional en la práctica del Trabajo Social en España. Partiendo de la evidencia de que el desgaste se da en la práctica de los profesionales del Trabajo Social (Figley, 1995, Adams, Boscarino, y Figley, 2006, Bourassa, 2009, Dekel y Baum, 2010, Campos y Cardona, 2017), consideramos que es necesario dotarse de perspectivas teóricas y prácticas alternativas a las tesis dominantes a cerca del desgaste.

El desarrollo de las relaciones de ayuda con las personas con las que trabajamos en distintos contextos implica el desarrollo de competencias y capacidades ligadas al manejo de nuestra capacidad empática y al desarrollo de una alianza de ayuda (Friedlander, Heatherington y Escudero, 2009, Cardona 2012). Es por ello, por el hecho de trabajar con nuestras capacidades de escucha, vinculándonos a personas que sufren psíquica, física o emocionalmente, 
por lo que se paga un precio conocido: desgaste físico, desgaste emocional y agotamiento de las capacidades y competencias profesiones. Comprender lo que les sucede a nuestros clientes, entender los significados de sus situaciones y vincularnos a su malestar genera dolor en el profesional. En otros términos, vincularse, duele (Campos y Cardona, 2017).

\section{Fuentes del malestar profesional}

Nos interesa poner en evidencia el fenómeno del desgaste profesional desde el punto de vista relacional. Así, consideramos que una relación de ayuda eficaz requiere del desarrollo de una relación de confianza entre el profesional y el cliente. Esta relación se construye mediante el despliegue de dos factores: En primer lugar nuestra capacidad de empatizar con el cliente. En condiciones de normalidad, se trata de nuestra capacidad psicofisiológica de comprender desde el punto de vista cognitivo y emocional lo que le sucede a nuestro cliente (Bermejo, 2012, Bermejo y Santos, 2015, Carpena, 2016). En segundo lugar, el desarrollo de una alianza para el cambio entre el profesional y el cliente. Ésta incluye cuatro dimensiones: lazos emocionales entre ambos y la confianza mutua, el respeto y el interés por el proceso de cambio, un compromiso activo por el proceso y un sentido de asociación para alcanzar un común objetivo (Friedlander, Escudero y Heatherington, 2009). Sin el desarrollo y la puesta en juego de la empatía, el trabajo social centrado sobre la persona es imposible (Adams et al, 2006).

Así, empatía y alianza serán las generadoras de tres significativas fuentes de desgaste. En primer lugar, es necesario contrarrestar nuestra reflejo empático espontáneo. Éste es irreflexivo e inconsciente, se traduce en una escucha desequilibrada de las narrativas que las personas nos cuentan y que están inundadas de problemas, malestar incapacidad o dolor. En estas narrativas, nuestra capacidad empática se dispara como un primer reflejo y se traduce en la tendencia a escuchar y empatizar, preferentemente, a aquel o a aquella historia en la cual percibimos resonancias personales, ideológicas culturales o de género, en las cuales hay buenos y malos, víctimas y victimarios. Es comprensible que, en tanto profesionales sensibles con las personas que sufren, tengamos la tendencia a empatizar con el perdedor, o con el que aparentemente lleva la peor parte. Dado que una buena escucha no es un juicio, corregir esta tendencia involuntaria requiere un esfuerzo que genera desgaste.

En segundo lugar, escucha activa y empatía implican el desarrollo de una actitud empática consciente. La actitud empática implica una predisposición consciente y voluntaria para comprender la posición del otro, el discurso del otro y el significado que el otro da a su discurso, sus emociones y sus vivencias. 
Para desarrollar una aprehensión de los significados, los profesionales deben percibir y experimentar parte del malestar que sienten las personas. Una escucha activa activada por una predisposición y una actitud empática, implica percibir en sí mismo parte del malestar del otro. Experimentar parte del dolor del otro, genera desgaste. Fue Figley (1995:24) el que por primera vez acuño el término Fatiga por Compasión haciendo referencia al «sentimiento de profunda empatía y pena por otro que está sufriendo, acompañado por un fuerte deseo de aliviar el dolor o de resolver sus causas». Una tercera fuente de desgaste está relacionada con la necesaria distancia emocional, por la tensión paradójica entre empatía y ecpatía. El concepto de ecpatía (González de Rivera, 2004) hace referencia a nuestra capacidad de controlar la reacción empática mediante la exclusión activa de los sentimientos inducidos por los demás. La práctica de la ecpatía nos protege de la inundación afectiva y del dejarnos llevar por las emociones de las partes mediante una forma de control intencional de la subjetividad interpersonal.

Por tanto, una adecuada práctica profesional requiere del despliegue de nuestra capacidad empática, de la alianza de ayuda y de la distancia emocional necesaria para la efectividad de la relación. Es evidente que esta tensión paradójica entre cercanía emocional y distancia emocional, genera más desgaste en un contexto de interacción con personas, parejas, familias o grupos que experimentan situaciones de sufrimiento físico, psíquico, relacional o emocional.

\section{Perspectivas diversas sobre el desgaste profesional. Revisión bibliográfica}

Hemos efectuado una revisión sobre fuentes bibliográficas de base nacional, centrándonos en revistas españolas cuya temática es específica sobre Trabajo Social y Servicios Sociales: Alternativas, Cuadernos de Trabajo Social, Azarbe, Educación Social, Humanismo y Trabajo Social, Comunitaria, Atlántida, Ehquidad, Portularia, Trabajo Social y Salud, Revista de Treball Social, Documentos de Trabajo Social, Trabajo Social hoy, Revista de Política Social y Servicios Sociales, Trabajo Social Global y TS Nova. Dada la limitación del universo de revistas, no ha sido necesario acudir a las bases de datos disponibles. Hemos realizado búsquedas introduciendo las siguientes palabras clave: $<$ traumatización secundaria>, <fatiga por compasión>, <desgaste por empatía $>$, < desgaste profesional $>$, <burnout $>$ y <autocuidado $>$, generando también búsquedas AND y OR en los casos del fatiga por compasión y desgaste por empatía. Aunque éramos sabedores de que el concepto de fatiga por compasión, de nuestro especial interés, probablemente no se vería reflejado, los resultados obtenidos en relación a la búsqueda han sido más escasos de los esperados. No existen trabajos relacionados con los conceptos de trauma, traumatización, 
traumatización secundaria, desgaste por empatía, empatía, compasión o fatiga por compasión. Hemos detectado seis trabajos relacionados directamente con el fenómeno del burnout, dos que abordan el desgaste y dos relacionados con el autocuidado. Vistos los resultados y revisada la bibliografía, no hemos seleccionado ninguno de los documentos hallados. Ello nos ha obligado a acudir a fuentes indirectas relacionadas con revistas de base nacional vinculadas a la medicina, la psicología y la enfermería. En ellas hemos observado que el abanico de documentos es amplio y diverso. Sin embargo, no proporcionaban información ni de los autores más importante que han investigado y descrito el fenómeno de la fatiga por compasión ni tampoco aparecen en las revistas españolas referencias de investigación en el marco de la práctica del Trabajo Social. Ello nos ha obligado a rastrear, inevitablemente, las bases de datos que, en el ámbito de las ciencias sociales y del Trabajo Social, son valoradas como de reconocido prestigio: Web of Science, SCOPUS, EBSCOhost, PsycoARTICLES Sciencedirec. Los resultados ponen en evidencia que el fenómeno de la fatiga por compasión, claramente diferenciado del concepto de burnout, está suficientemente recogido en las principales revistas del trabajo social anglosajón. Desarrollamos a continuación los resultados obtenidos

La Psicotraumatología es la disciplina que con más relevancia ha centrado sus esfuerzos en investigar y conocer el alcance y el impacto que las relaciones de ayuda ejercen sobre el equilibrio físico, psíquico y emocional de los profesionales que ejercen dichos roles (Orengo, 2002). Los antecedentes del estudio del impacto de la relación de ayuda sobre los profesionales los podemos encontrar en los trabajos de C. Figley (1995), el cual estudia por primera vez estas consecuencias con enfermeras que atendieron veteranos de la guerra de Vietnam en la década de los 60. Según el autor, estas profesionales tenían sentimientos de culpa y remordimiento por no haber podido «salvar» a sus pacientes. Joison (Jiménez et al, 2004) utilizó el término fatiga por compasión para referirse al efecto nocivo de la exposición continuada de las enfermeras a las historias de dolor y sufrimiento de sus pacientes. Cabría preguntarse qué es la fatiga por compasión y que efectos nocivos genera en aquellos que la experimentan. Se ha definido la fatiga por compasión como «el estado de agotamiento y disfunción biológica, psicológica y social, resultado de la exposición prolongada al estrés por compasión y todo el que ello evoca» (Lynch y Lobo, 2012:21-25). Para Figley (2002: 14-30), «la fatiga por compasión como cualquier otra forma de fatiga, reduce nuestra capacidad o nuestro interés en soportar el sufrimiento de los demás». Si un profesional experimenta dicho fenómeno, su capacidad para empatizar, conectar y ayudar a sus clientes se ve gravemente disminuida. Figley (2014) considera a la fatiga por compasión como las conductas y reacciones emocionales naturales derivadas de conocer 
un evento traumático experimentado por una persona significativa, o sea, una traumatización indirecta de instauración rápida y repentina como consecuencia de ayudar a aquellos que experimentan el trauma de manera directa y/o primaria.

Los últimos trabajos publicados abandonan la visión restringida aportada por la psicotraumatología y amplían el concepto de fatiga por compasión entendiéndola como el resultado progresivo y acumulado de un intenso contacto con las personas que sufren y por la exposición al estrés y que incluyen a todos los profesionales que desarrollan una relación de ayuda (Adams, Boscarino y Figley, 2006, Figley y Bride, 2007, Figley, Adams, y Boscarino, 2008, Dekel y Baum, 2010). Hemos rastreado el concepto de fatiga por compasión en las revistas científicas de la categoría «Trabajo Social» de la Web of Science y Scopus. El rastreo de las publicaciones pone en evidencia el manejo del concepto de fatiga por compasión en el mundo del Trabajo Social anglosajón (Compassion Fatigue). Hemos rastreado el tema del desgaste profesional en los números de las revistas científicas de Trabajo Social de los últimos diez años, encontrando únicamente referencias al tema del burnout, y ninguna relacionada con fatiga por compasión o, en su defecto, de Estrés Traumático Secundario. Es por ello que afirmamos que el concepto de fatiga por compasión o desgaste por empatía, es inexistente en la literatura científica del trabajo social en España. El denominador común de todas las propuestas relativas a su definición descansa en el concepto de empatía, no en el concepto de trauma.

Es una evidencia que en un sistema abierto y vivo como es el sistema de ayuda formado por el trabajador social y su cliente, se produce un permanente intercambio de información, relaciones e influencias mutuas. En función de las características del contexto de intervención para el cambio (Cardona y Campos, 2009, Cardona, 2012), la relación de ayuda está sesgada por un conjunto de relaciones y emociones intensas que afectan a todos los partícipes del proceso, y que se manifiestan a través de la ansiedad, el estrés y las emociones expresadas y sentidas por todos los participantes. La dimensión fisiológica (Casado, 2004) vinculada al estrés y la ansiedad de los trastornos que se experimentan cuando se está sumido en un contexto relacional y emocional intenso y en ocasiones tóxico, nos permite aproximarnos al conjunto de ideas clave: el trabajo que desarrollan los trabajadores sociales genera un desgaste físico y mental inevitable dada la naturaleza del contexto y de las emociones en el que se desenvuelven: conflicto, tensión, estrés y dolor emocional. En consecuencia, desarrollar el rol de trabajador social conlleva un coste relacionado con la erosión y el desgaste de las capacidades y competencias. 


\section{Signos de la Fatiga por Compasión}

A diferencia del burnout, entendido este como el resultado de un proceso acumulativo y crónico relacionado con las condiciones de trabajo, la fatiga por compasión puede aparecer como un proceso agudo, súbito e inmediato tras una relación de ayuda con un cliente. Campos Méndez (2015), siguiendo a Figley (1995; 2002) y Sabo (2011), hacen referencia a un inicio brusco (agudo) en el cual podemos identificas tres momentos: 1. Disconfort por Compasión, con la aparición de causas que provocan el cambio hacia el malestar; 2. Estrés por Compasión en progresivo aumento, superando los umbrales de tolerancia y, 3. fatiga por compasión. En conjunto, los signos de la fatiga por compasión se estructuran en tres ejes coincidentes con los del estrés traumático secundario: la hiperactivación, la evitación y la re-experimentación.

Las manifestaciones sintomáticas de la fatiga por compasión han sido resumidas por Campos Méndez (2015), a partir de las aportaciones de Figley (2002), Coetzee y Klopper (2010), Moreno-Jiménez (2004) y Gilmore (2012).

Estas se estructuran en cinco dimensiones de la vida personal y profesional del trabajador social:

1. Reacciones somáticas centradas en el agotamiento y pérdida de fuerza, falta de energía., disminución de la eficacia, desempeño reducido, pérdida de resiliencia, recuperación física lenta y más costosa, aumento de quejas físicas, propensión a accidentes, insomnio, dolores de cabeza, malestar estomacal, tensiones musculares, aceleración del ritmo cardíaco, temperatura corporal fluctuante y dolor de espalda.

2. Reacciones relacionales como la insensibilidad y la indiferencia, incapacidad para abordar o paliar el sufrimiento y la ausencia de disfrute con las actividades de ocio.

3. Reacciones emocionales como la pérdida de entusiasmo y apatía, desensibilización, ira o irritabilidad intensa, deseo de abandonar, desbordamiento emocional, sentimientos de falta de gozo en actividades cotidianas, sensación de vacío o desesperanza hacia futuro, estado de depresión o tristeza la mayor parte del tiempo, miedo y ansiedad ante eventos, sentimientos de paralización, falta de motivación, compulsiones como beber, comer y gastar en exceso.

4. Reacciones espirituales como la falta de consciencia espiritual, el desinterés por la introspección, juicios empobrecidos, merma en el discernimiento y cuestionamiento de creencias espirituales. 
5. Reacciones cognitivas tales como el aburrimiento y el desinterés para con los clientes, desorganización, atención disminuida, menor capacidad de concentración, sueños y pesadillas recurrentes y problemas de concentración.

Tabla I. Características y signos de la Fatiga por Compasión

\begin{tabular}{|c|c|c|}
\hline Hiperactivación & Evitación & Reexperimentación \\
\hline $\begin{array}{l}\text { - } \text { Problemas de sueño } \\
\text { - Irritabilidad } \\
\text { - Estallidos de ira } \\
\text { - Hipervigilancia } \\
\text { - Incremento de ansiedad } \\
\text { - } \text { Reactividad } \\
\text { - Aumento de percepción } \\
\text { de exigencias y de } \\
\text { amenazas } \\
\text { - Aumento de la } \\
\text { frustración }\end{array}$ & $\begin{array}{l}\text { - «No querer volver ahí } \\
\text { otra vez» } \\
\text { - Deseo de evitar los } \\
\text { pensamientos } \\
\text { - Evitación de } \\
\text { sentimientos } \\
\text { - Evitación de } \\
\text { conversaciones asociadas } \\
\text { - al dolor del cliente } \\
\text { - Evitación a la exposición } \\
\text { del material traumático } \\
\text { del cliente } \\
\text { - Pérdida de energía } \\
\text { - Evitación de salidas } \\
\text { extra-laborales con los } \\
\text { compañeros } \\
\text { - Auto-medicación y/ } \\
\text { adicciones }\end{array}$ & $\begin{array}{l}\text { - Pensamientos intrusivos } \\
\text { - Sueños intrusivos } \\
\text { - Distrés psicológico y/o } \\
\text { fisiológico } \\
\text { - Pensamientos e } \\
\text { imágenes asociadas con } \\
\text { experiencias traumáticas } \\
\text { de los clientes } \\
\text { - Pensamientos y } \\
\text { sentimientos de falta } \\
\text { de idoneidad como } \\
\text { profesional asistencial }\end{array}$ \\
\hline
\end{tabular}

Fuente: Campos Méndez, 2015

\section{Fatiga por compasión y otras formas de desgaste}

Asociado a los «costes del cuidado» a otras personas que padecen y sufren, numerosos términos utilizados han hecho posible una cierta confusión teórico-conceptual (Acinas, 2011). Como ya señaló Figley (Figley, 1995, 2002, Figley y Bride, 2007, Figley, 2014) se han usado diversos términos para hacer referencia al desgaste que describe la actual acepción de la fatiga por compasión. Nos referimos a los conceptos de estrés traumático secundario (estrés traumático derivado del contacto con la víctima o el evento traumático), al Estrés Post-traumático, a la Traumatización Vicaria, la Contratransferencia y también el conocido Síndrome de Burnout (Lynch y Lobo, 2012), así como el contagio emocional (Fernández-Pinto, López-Pérez, y Márquez, 2008). A excepción del burnout, todos ellos se manifiestan en las actividades vinculadas con personas víctimas de un evento traumático. El estrés traumático secundario se produce en familiares o personas cercanas de la persona que sufre un 
acontecimiento traumático como consecuencia de la proximidad de la relación y también como el impacto que genera en los profesionales de la relación de ayuda al observar o escuchar el relato de una persona traumatizada de forma primaria (Rothschild, 2006). La traumatización vicaria hacen referencia a una transformación de los esquemas cognitivos y sistemas de creencias que resultan de compromiso empático con las experiencias traumáticas de los clientes (Pearlman y Saakvitne, 1996:151). Y finalmente, la fatiga por compasión, es definido por Figley (1995) como el sufrimiento (no traumatización) producido en el profesional como consecuencia de su trabajo en un servicio de apoyo a personas que presentan un sufrimiento o disfuncionalidad, no necesariamente traumatizadas.

En ocasiones se ha considerado a la fatiga por compasión como una subtipología del síndrome de burnout. Diversos autores (Najjar, Davis, Beck-Coon, y Carney-Doebbeling, 2009) consideran que la fatiga por compasión es una forma única de burnout, en la cual la causa del malestar no está tan relacionada con los factores estresantes del trabajo como con la respuesta empática que los profesionales que trabajan con la empatía proporcionan. Sabo $(2006,2011)$ describe el síndrome de burnout como un proceso gradual resultado de las respuestas que damos en nuestro entorno de trabajo, mientras que la fatiga por compasión tiene un inicio agudo que resulta de la atención a las personas que están sufriendo, producto de la experiencia de hacerse cargo, empáticamente, del malestar del otro, estando solo está presente en las profesiones relacionadas con las relaciones de ayuda (Jacobson, Rothschild, Mirza y Shapiro, 2013, Stamm, 2002). La fatiga por compasión es la consecuencia natural, predecible, tratable y paliable de trabajar con personas que sufren; es el residuo emocional resultante de la exposición al trabajo con aquellos que sufren las consecuencias de eventos traumáticos (Acinas, 2012, Rothschild, 2006).

\section{Evaluación de la Fatiga por Compasión}

Los instrumentos de medida de la fatiga por compasión son escasos. En 1995 C. Figley y B. H. Stamp construyen el Compassion Fatigue and Satisfaction Test (Cuestionario Autoaplicado de Fatiga de Compasión y Satisfacción; Figley y Stamm, 1995). La evolución de este instrumento se materializa hoy en día en el la información sobre la revisión del trabajo anterior de Figley y Stamm (1995) desarrollada por Stamm, Larsen y Davis (2002). Como resultado, a día de hoy disponemos del Professional Quality Life Scale, ProQOL, versión V (Stamp, 1997) que está formada por 30 ítems que se distribuyen en tres subescalas: estrés traumático secundario, burnout y satisfacción de compasión. La dimensión de Satisfacción por Compasión recoge la medición de la satisfacción 
generada el en profesional por el hecho de hacer bien el trabajo. La dimensión de estrés traumático secundario mide los efectos de la exposición al trauma o situaciones estresantes por parte del profesional, mientras que la dimensión de burnout mide el agotamiento experimentado a través de la expresión de ideas y sentimientos negativos de desesperanza y despersonalización.

Estimaciones de la fiabilidad de consistencia interna para las subescalas son reportados con un Alfa de Cronbach 0,87 para la escala de satisfacción compasión, 0,72 para la escala de burnout, y 0,80 para la escala trauma secundario fatiga de la compasión (Bride, Radey y Figley, 2007).

Los último avances en la mediación de la fatiga por compasión podemos encontrarlos en los trabajos de Zamponi, Viñuela, Rondoni, Tauber y Main (2009). El Inventario de Desgaste por Empartía (IDE) consta de 27 ítems con un Alpha de Cronbach de 0,7 que se organizan en tres dimensiones. La primera dimensión hace referencia a la Involucración Profesional, es decir, el grado de involucración del profesional en la relación entre profesional y cliente, considerando que una buena práctica profesional significa un grado razonable y seguro de involucración. La segunda dimensión hace referencia al Cuidado Personal, es decir, en que medida el profesional cuenta con recursos personales y técnicos para afrontar los riesgos y el desgaste psicofísico derivado de la relación de ayuda. El tercer factor hace referencia al grado de Vulnerabilidad, reflejando en qué medida el profesional se percibe afectado por su práctica profesional, manifestándose y reconociéndose a nivel relacional, psiquico, físico y emocional. En base a las puntuaciones obtenidas y a a combinación de puntuaciones de cada dimensión, el IDE identifica cuatro perfiles posibles: Perfil I, Funcionamiento empático óptimo, sin riesgo de contraer Síndrome de Desgaste por Empatía; Perfil II, Funcionamiento empático normal, sin riesgo de contraer síndrome de desgaste por empatía; Perfil III, Funcionamiento empático normal, con riesgo de contraer síndrome de desgaste por empatía, y Perfil IV, Funcionamiento Empático Disfuncional. A nuestro juicio, y a diferencia de la escala ProQOL, el constructo desgaste por empatía está más definido en el Inventario de Desgaste por Empatía. Las dimensiones del cual se componen facilitan no solo el conocimiento del nivel de desgaste en alguno de sus cuatro perfiles, sino también el conocimiento de cuál es la dimensión más significativa a efectos de valorar el desgaste. En términos pragmáticos, conocer la manifestación del desgaste en cada una de las dimensiones facilita la aplicación de medidas concretas que apuntan a las dificulades identificadas en cada dimensión. 


\section{Modelo general de Resiliencia a la Fatiga por Compasión}

Los trabajos de Figley $(2006 ; 2014)$ insisten en la búsqueda de un modelo de interpretación de la fatiga por compasión que permita determinar cuáles son las variables del cual depende. En este proceso se ha considerado que el fenómeno de la fatiga depende del comportamiento de seis factores. El modelo general de resiliencia a la fatiga por compasión descrito por Figle (2014), se compone de los siguientes factores y de la singular relación entre ellos:

1. Exposición al cliente que sufre: cuanta más exposición al cliente que sufre mayor es la respuesta empática, cuanto mayor es el estrés por compasión residual, menor es la resiliencia a la fatiga por compasión.

2. Habilidad empática: es el grado en que el profesional puede predecir de forma ajustada la emoción expuesta por el cliente y exponer emociones al cliente que reflejan servicios efectivos y con resultados.

3. Preocupación por el cliente: es el interés en el bienestar y el éxito del cliente. Motiva al profesional a buscar la mejor respuesta terapéutica.

4. Respuesta terapéutica: es la que el profesional utiliza para permitir al cliente a) tener más voluntad para cambiar, b) tener menos temor, e) ser más optimista acerca del resultado de la intervención, d) sentirse apoyado. Sin embargo, dando la respuesta terapéutica adecuada, el trabajador experimenta distrés vicario del cliente que carga en el profesional, de forma medible, como Estrés por Compasión Residual.

Figura I. Modelo general de resiliencia a la Fatiga por Compasión

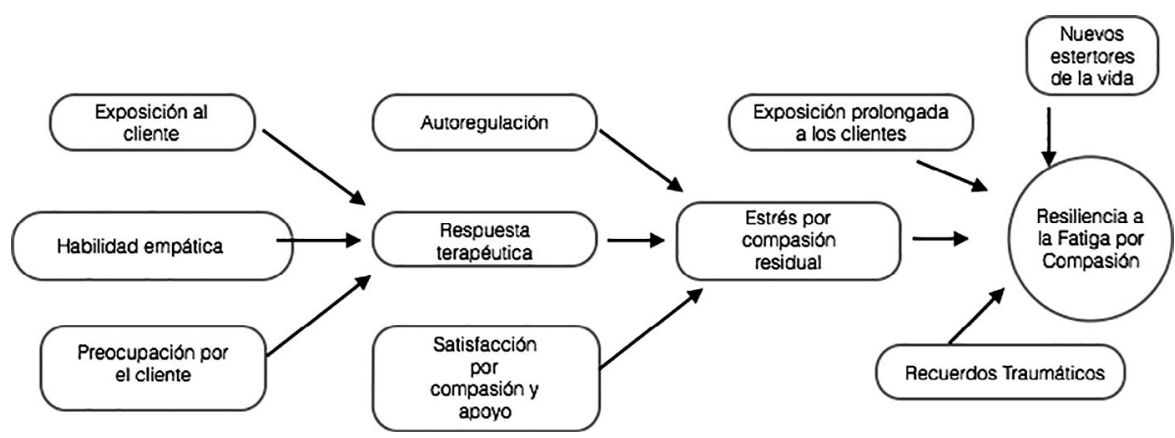

Fuente: Figley 2014

5. Auto-regulación: es el grado por el cual el profesional puede manejar de forma efectiva la causa y el impacto del estrés y separar el trabajo 
de la vida personal; sentirse fresco cada día gracias a la habilidad del trabajador de auto-regularse.

6. Satisfacción por compasión y apoyo: es el grado en que un profesional siente un alto nivel de satisfacción personal por el trabajo con sus clientes y percibe un alto grado de nivel de apoyo y respeto por los compañeros de trabajo.

7. El estrés residual por compasión: es experimentado por los profesionales como reacciones de estrés a la situación difícil del cliente y la preocupación de hacer todo lo que uno pueda para ayudar al cliente que está sufriendo. Adicionalmente a la respuesta terapéutica, la autorregulación y la satisfacción por compasión y apoyo también afecta al nivel de estrés residual por compasión.

8. Resiliencia a la fatiga por compasión: es la velocidad y grado de recuperación total de una adversidad del profesional después de haber experimentado un significativo incremento en el volumen de estrés. El modelo sugiere que es una función en cuatro variables diferentes: Estrés por compasión residual, exposición prolongada a los clientes, manejo efectivo de los recuerdos traumáticos (si hay alguno) y nuevos estresores de la vida.

8.1. La exposición prolongada a los clientes es el número de clientes asignados versus el tiempo al día para proveer servicios multiplicado por el número de meses realizando estas tareas.

8.2. El manejo efectivo de recuerdos traumáticos es la satisfacción, auto-reportada, manejando el número (si hay) y la intensidad de los recuerdos del trauma pasado. Mientras mayor sea la satisfacción en manejar los recuerdos, mayor es la resiliencia a la fatiga por compasión. No es importante solo para predecir la resiliencia a la fatiga por compasión, también para predecir como de bien el profesional utiliza las lecciones del trauma pasado para ayudar a los clientes que están experimentando un trauma similar y para adaptarse al trauma.

8.3 Nuevos Estresores de la Vida: Acontecimientos propios del desarrollo del ciclo vital o de acontecimientos no previstos de que provocan crisis personales y familiares que afectan a los profesionales en cuanto personas.

\section{¿Cómo paliar la Fatiga por Compasión?}

Como fenómeno inherente al proceso de ayuda, la fatiga por compasión no es prevenible. Su existencia y manifestación refleja el desarrollo de un buen 
enganche relacional y un adecuado uso de nuestra capacidad empática. Ahora bien, ello no significa que no podamos desarrollar acciones conscientes para minimizar sus efectos.

Bride, Radey y Figley (2007) señalan que la prevalencia de la fatiga por compasión es el resultado, siempre desequilibrado, del balance entre la satisfacción por compasión y la fatiga por compasión. La Satisfacción por Compasión está relacionada con la percepción subjetiva del trabajo bien hecho, de la conexión emocionalmente significativa con nuestros clientes y la satisfacción de las necesidades psicoemocionales del propio profesional. Sin embargo es sabido que este balance, de inclinarse peligrosamente hacia un aumento significativo de la fatiga por compasión, incrementa el riesgo de desconexión emocional, el peligro de hacer juicios erroneos o de organizar deficientemente y proceso de cambio. Desde esta perspectiva, el coste de cuidar debe ser compensado con el autocuidado. Podemos entender por autocuidado como «aquellas actividades que realizan los individuos, las familias o las comunidades, con el propósito de promover la salud, prevenir la enfermedad, limitarla cuando existe o restablecerla cuando sea necesario.» (Ginés y Carvalho, 2010:307), teniendo dimensión institucional, personal o comunitaria. Si esta compensación no se da, la fatiga por compasión prevalecerá a la satisfacción por compasión. Bride, Radey y Figley (2007) proponen que paliar la fatiga por compasión, decir, contrabalancear el desequilibrio estructural que siempre existirá entre satisfacción y fatiga, significa desarrollar cuatro áreas (Campos y Cardona, 2017).

a. Desarrollo de relacionales positivas con los clientes, es decir, activar permanentemente la respuesta empática y las dimensiones de la alianza de ayuda. Se trata, resumiendo, de trabajar bien, conscientemente bien. También apuntan a cubrir las necesidades del profesional tales como la satisfacción por la relación prestada o el reconocimiento de los colegas.

b. Autocuidado inespecífico relacionado con el mantenimiento de estándares reconocidos de autocuidado que definen el estilo de vida (descansar, dormir y comer bien, mantener relaciones y redes, aficiones, etc.). En necesario monitorizar frecuentemente el cumplimiento del plan (consciente) de auto-cuidado y actualizarlo periódicamente.

c. Autocuidado intencional vinculado a la permanene revisión de la práctica profesional y su impacto sobre si mismo, recurriendo a la supervisión, revisión de la práctica, participación en foros profesionales, estudio, equilibrio biofísico (alimentación, ejercicio, descanso y placer).

d. Estrategias de manejo del estrés mediante el cuidado y desarrollo de aficiones, relajación, yoga, meditación consciente (mindfulness), expresiones artísticas, escritura terapéutica, etc. 
Para concluir, queremos insistir en la dimensión positiva de la fatiga por compasión. Ésta refleja un claro compromiso del profesional respecto a su cliente y a su propia y adecuada práctica. Es necesario e inevitable si se desarrolla una buena práctica; es inhabilitante si no se compensa mediante otros mecanismos: relaciones positivas, autocuidado y estrategias de manejo del estrés. La fatiga por compasión no se puede prevenir pero puede y debe paliarse de forma consciente. Incrementar la capacidad de reconocer y minimizar el impacto del desgaste es una responsabilidad del profesional y de la organización en la cual trabaja.

\section{Referencias}

ACINAS, M. P. (2012). Burn-out y desgaste por empatía en profesionales de cuidados paliativos. Revista Digital de Medicina Psicosomática y Psicoterapia, 2(4), 1-22.

ADAMS, R., BOSCARINO, J. y FIGLEY, C. (2006). Compassion fatigue and psychological distress among social workers: a validation study. The American journal of orthopsychiatry. Vol 76(1), 103-108. https://doi.org/10.1037/0002-9432.76.1.103

Barrera Algarín, E., Malagón Bernal, J. y Sarasola Sánchez- Serrano J. (2015). Trabajo Social, su contexto profesional y el Síndrome de Burnout. Comunitania. Revista Internacional de Trabajo Social y Ciencias Sociales, 9, 51-71. https://doi.org/10.5944/comunitania.9.2

Blanco Montilla, M. (2006). Estudio Cualitativo sobre el Síndrome de Burnout en el Trabajo Social. Acciones e investigaciones sociales, Extra (1), 443

BERMEJO, J.C. (2012). Empatía Terapéutica. La compasión del sanador herido. Bilbao: Desclée de Brouwer.

Bermejo, J.C. y SANTOS, E. (2015). Counselling y cuidados paliativos. Bilbao: Desclée de Brouwer.

Bride, B. E., Radey, M., y Figley, C. R. (2007). Measuring compassion fatigue. Clinical Social Work Journal, 35, 155-163. https://doi.org/10.1007/ s10615-007-0091-7

Bourassa, D. B. (2009). Compassion fatigue and the adult protective services social worker. Journal of Gerontological Social Work, 52(3), 215-229. https:// doi.org/10.1080/01634370802609296

Campos, J.F., Cardona, J., Cuartero, M.E. y Bolaños, I. (2015). La Fatiga por Compasión en la Práctica de la Mediación Familiar. En (a cura di) Danilo De Luise e Mara Morelli, Voci dal X Congresso Mondiale di Mediazione: una via verso la cultura della pace e la coesione sociale. Genova: Editrice ZONA.

CAMPOS, J.F. y CARDONA, J. (2017). El dolor del vínculo: la Fatiga por Compasión. En Guinot, C y Ferran, A. (eds). Trabajo Social: arte para generar vínculos. Bilbao: Publicaciones de la Universidad de Deusto, 229-240. 
CAMPOS MÉndez, R. (2015). Estudio sobre la prevalencia de la fatiga de la compasión y su relación con el síndrome de «Burnout» en profesionales de Centros de mayores en Extremadura. Tesis Doctoral. Cáceres: Universidad de Extremadura.

CARDONA, J. y CAMPOS J.F. (2009). Cómo determinar un contexto de intervención: Inventario para el análisis de la relación de ayuda entre el trabajador/a social y el cliente durante la fase de estudio y evaluación de la situación problema. Portularia 9(2), 17-35

Cardona, J. (2012). La definición del Contexto de Intervención en el Trabajo Social de Casos. Tesis Doctoral. Palma de Mallorca:Univeresitat de les Illes Balears.

Carpena, A. (2016). La empatía es posible. Bilbao: Desclée de Brouwer.

Casado, M.I. (1994). Ansiedad, Stress y Trastornos Psicofisiológicos. Tesis Doctoral. Madrid: Universidad Complutense.

Coetzee, S. K., y Klopper, H. C. (2010). Compassion fatigue within nursing practice: a concept analysis. Nursing $\&$ Health Sciences, 12(2), 235-43. https:// doi.org/10.1111/j.1442-2018.2010.00526.x

DEKEL, R. y BAUM, N. (2010). Intervention in a shared traumatic reality: A new challenge for social workers. British Journal of Social Work, 40(6), 1927-1944. https://doi.org/10.1093/bjsw/bcp137

De la Fuente RoldÁn, I. y SÁNCHEZ Moreno, E. (2012). Trabajo social, síndrome de estar quemado por el trabajo y malestar psíquico: un estudio empírico en una muestra de trabajadores sociales de la comunidad de Madrid. Portularia: Revista de Trabajo Social, Extra, 12, 121-130. https://doi.org/10.5218/prts.2012.0013

FACAL FONDO,T. (2012). Prevalencia del Síndrome de Burnout en trabajadores de los servicios sociales comunitarios. Portularia 12(1), 59-69. https://doi. org/10.5218/prts.2012.0034

FERnÁNDEZ-Pinto, I., LÓPEZ-PÉREZ, B. y MÁrquez, M. (2008). Empatía: Medidas, teorías y aplicaciones en revisión. Anales de Psicología, 24(2), 284-298.

Friedlander, M. L., HeATHERINGTON, L., y Escudero, V. (2009). La alianza terapéutica: En la terapia familiar y de pareja. Barcelona: Paidós

Figley, C. (1995). Compassion Fatigue: Coping With Secondary Traumatic Stress Disorder In Those Who Treat The Traumatized. U.K.: T. \& Francis, Ed.

FIGLEY, C (2002). Compassion fatigue: psychotherapists' chronic lack of self care. Journal of clinical psychology, 58(11), 1433-41. https://doi.org/10.1002/ jclp. 10090

Figley, C. y BRIDE, B. (2007). The Fatigue of Compassionate Social Workers: An Introduction to the Special Issue on Compassion Fatigue. Clinical Social Work Journal, 35(3),151-153. https://doi.org/10.1007/s10615-007-0093-5

Figley, C., ADAMS, R. y BosCARINO, J. (2008). The compassion fatigue scale: its use with social workers following urban disaster. Research on Social Work Practice, 18 (3), 238-250. https://doi.org/10.1177/1049731507310190 
FIgley, C. (2014). A Generic Model of Compassion Fatigue Resilience (c). Retrieved November 20, 2014, from http://figley.blogspot.com.es/

Ginés, O., y Carvalho, B. (2010). Cuidados con el equipo cuidador. Revista Brasileira de Psicoterapia, 12(2-3), 297-313.

GILmore, C. (2012). Compassion fatigue, what it is and how to avoid it. Nursing N.Z 18 (5), 32.

GonZÁleZ DE RiverA, J.L. (2004). Empatía y Ecpatía. Psiquis 25(6), 243-245

JACOBSOn, J.M., Rothschild, A., MirzA, F. \& SHAPIRO, M. (2013). Risk for burnout and compassion. Journal of Social Service Research, 39 (4), 455-468. https:// doi.org/10.1080/01488376.2012.744627

Jiménez, B., Eugenia, M., Benadero, M., Novoa, L., Carvajal, R., Hernández, E. G., y Moreno-JimÉnEZ, B. (2004). Estrés Traumático Secundario. Evaluación, prevención e intervención. Terapia Psicológica, 22(1), 69-76.

LÁZARO FERnÁNDEZ, S.(2004). El desgaste profesional (síndrome de Burnout) en los trabajadores sociales. Portularia 4 (4), 99-506.

LYNCH, S. H., \& LOBO, M. L. (2012). Compassion fatigue in family caregivers: a Wilsonian concept analysis. Journal of Advanced Nursing, 68(9), 2125-34. https://doi.org/10.1111/j.1365-2648.2012.05985.x

MASLACH, C. \& JACKSON, S (1986). Maslach burnout inventory manual. Palo alto, CA: Consulting Psychologist Press, Inc.

NajJar, N., Davis L.W., BeCK-CoOn, K. \& CARney-Doebbeling, 2009(2009). Fatigue: A Review of the Research to Date and Relevance to Cancercare Providers. Journal of Health Psychology, 14 (2), 267-27. https://doi. org/10.1177/1359105308100211

FReUdenberger, H. J. (1974). Staff burnout. Journal of Social Issues, 30(1), pp. 159-165. https://doi.org/10.1111/j.1540-4560.1974.tb00706.x

Orengo García, F. (2002) Sobre Psicotraumatología. Psiquis 23 (5),173-177.

Pearlman, Laurie A., \& SaAkvitne, K. W. (1996). Trauma and the Therapist: Countertransference and Vicarious Traumatization in Psychotherapy with Incest Survivors. New York: W.W. Norton,

RothsCHILD, B. (2006). Help for the Helper: The Psychophysiology of Compassion Fatigue and Vicarious Trauma. New York: W.W. Norton \& Company, Ed.

SABO, B. (2011). Reflecting on the concept of compassion fatigue. Online journal of issues in nursing, 16 (1).

Stamm, H. (2009). Professional Quality of Life: Compassion Satisfaction and Fatigue Version 5 (ProQOL). En www.proqol.org

Zamponi, J., Rondón, JuAn MANuel y Viñuela, M. Agustina (2009). Primer cuestionario validado para evaluar Síndrome de desgaste por Empatía. Comunicación presentada en el III Congreso Iberoamericano de Psicología del Trabajo. Buenos Aires, Argentina.

Alternativas. Cuadernos de Trabajo Social, 24, 2017, pp. 119-136 - ISSN 1133-0473 
Cita bibliográfica: Morro Fernández, L. (2017). Factores sociofamiliares y estancia hospitalaria: la complejidad de la intervención social en el área de la psiquiatría de agudos. Aplicación de la Escala ECISACH-BCN PSMAR. [Family and social factors and hospital stay: the complexity of social intervention in the area of acute psychiatry. Application of the ECISACH-BCN PSMAR Scale]. Alternativas. Cuadernos de Trabajo Social, 24, 137-160. https://doi.org/10.14198/ ALTERN2017.24.08

\title{
FACTORES SOCIOFAMILIARES Y ESTANCIA HOSPITALARIA: LA COMPLEJIDAD DE LA INTERVENCIÓN SOCIAL EN EL ÁREA DE LA PSIQUIATRIA DE AGUDOS APLICACIÓN DE LA ESCALA ECISACH-BCN PSMAR
}

\author{
FAMILY AND SOCIAL FACTORS AND HOSPITAL STAY: \\ THE COMPLEXITY OF SOCIAL INTERVENTION IN THE AREA OF \\ ACUTE PSYCHIATRY \\ APPLICATION OF THE ECISACH-BCN PSMAR SCALE
} LAURA MORRO FERNÁNDEZ (iD
Hospital del Mar INAD-PSMAR. Passeig Marítim 25-29 Barcelona 08003 91135@parcdesalutmar.cat

\section{Resumen}

Estudiar la complejidad de los determinantes sociales en los procesos de hospitalización psiquiátrica puede ayudar a los profesionales de la salud mental a planificar los recursos que la población requiere durante el ingreso, así como planificar el alta hospitalaria. Hay factores de protección y de riesgo social que hemos de tener en cuenta en el momento de la intervención clínica y social, y que pueden determinar el pronóstico de la enfermedad. Este estudio tiene como objetivo describir los factores sanitarios, sociales y familiares que determinan la complejidad de la intervención social mediante la aplicación de la Escala ECISACH-BCN PSMAR en el área de hospitalización del servicio de psiquiatría del Hospital del Mar de Barcelona, mediante un estudio descriptivo correlacional. Los resultados nos sugieren que la falta de apoyo familiar complica las estancias hospitalarias, teniendo una relación franca la complejidad social y la duración del ingreso hospitalario.

Palabras clave: Complejidad, trabajo social, hospital, planificación del alta, estancia hospitalaria, apoyo familiar.

\begin{abstract}
Studying the complexity of social determinants in psychiatric hospitalization processes can help health professionals plan the resources required by the population during admission, as well as plan for hospital discharge. There are protection and social risk factors that must be considered at the time of clinical and social intervention which can determine disease prognosis. This is a descriptive correlational study that aims to describe the health, social and family factors that determine the complexity of social intervention through applying the ECISACH-BCN PSMAR Scale in psychiatric hospitalization at the Hospital del Mar, Barcelona. The study results suggest that the lack of family support complicates hospital stays and is related to social complexity and length of hospitalization.
\end{abstract}

Keywords: Complexity, social work, hospital, hospital discharge, hospital stay, family support. 


\section{Extended abstract}

\section{Background}

Studying the complexity of social determinants in psychiatric hospitalization processes can help health professionals plan the resources required by the population during hospital admission, as well as plan for discharge.

There are protection and social risk factors that must be considered at the time of clinical and social intervention that can determine disease prognosis.

This is a descriptive correlational study that aims to describe the health, social and family factors that determine the complexity of social intervention through applying the ECISACH-BCN PSMAR Scale in psychiatric hospitalization at the Hospital del Mar, Barcelona.

\section{Introduction}

\section{Health and social determinants}

Throughout history the meaning of the concept of health has changed over time. While the principle of health once referred to the absence of disease, beginning in the middle of the 20th century the concept was expanded upon, and the concept of health has come to refer to a state of complete physical, mental and social welfare.

In 1974, a Canadian report was published that defined the social determinants of health and described how social factors influence the improvement or impairment of population health.

The change in the perception of the concept of health has resulted in changes in the health, economic and social policies of countries, which have begun to take into account the ways in which social determinants affect health and people's lives.

If new models of health also apply to mental health and the treatment of mental health problems, we can say that mental health is something more than the absence of disorder; it is also an integral part of overall health. We thus consider that there is no health without good mental health.

\section{The intervention of medical social work in psychiat-} ric hospitalization

The study of the influence of social factors on hospital care is important for health professionals and administrators in order to measure the factors that affect hospital stays and to properly administer available resources.

Medical social work is a specialty of that has been developed and carried out within the health system (in primary care, social animal care or specialty care) with the objective of facilitating good health, preventing disease and helping the sick and their families to resolve disease-related social and psychosocial problems.

\section{The ECISACH-BCN PSMAR Scale}

Medical Social Work did not have an instrument to measure the complexity of social intervention until in 2012, when a group of medical social workers at the Central Hospital of Lisbon (ordinated by Dr. Serafim) applied the theories of Edgar Morin (regarding the paradigm of complexity) to intervention. They created an instrument that identifies the complexity of an intervention process according to a concrete social situation: the Scale of Social Intervention Complexity in Adults in a Hospital Context (ECISACH).

After the publication of the validation study of ECISACH in Aghatos Magazine, social workers at PSMAR decided to translate and validate the scale in the Spanish context with the name ECISACH-BCN PSMAR. The main objectives of this work follow.

\section{Objectives}

Measure the social intervention complexity in psychiatric hospitalization by investigating factors of mental illness in the family and social context to determine their gravity.

Secondary objectives include:

- Describe the family and social reality of patients admitted in psychiatry.

- Apply the ECISACH-BCN PSMAR scale in the field of psychiatry.

- Explore whether the information provided by the scale is a good measurement of hospital management.

The development of this study was based on two typical assumptions:

- First hypothesis: There is a relationship between the complexity of the intervention and the duration of the hospital stay.

- Second hypothesis: The family serves as a protective factor in relation to the complexity of a social intervention: patients with families present less complexity of social intervention in hospitals, and likewise, patients without families present greater complexity.

\section{Methods}

This is a correlational, descriptive, transversal study, with temporal variables.

The study sample is composed of patients admitted to the acute psychiatry units at Hospital del Mar during the period from January 2017 to May 2017 and that required social intervention.

The data are included in a database made up of one thousand items; it includes sociodemographic data and the twelve items of ECISACH-BCN PSMAR.

Statistical analysis was performed using SPSS. 


\section{Results}

The study results are presented in two areas:

1.- Descriptive analysis of the subscales of the ECISACH-BCN PSMAR Scale: related to the family relationship, $36.1 \%$ of the patients have a good family relationship, the rest presents family distort, being a high percentage, almost $23 \%$, the patients isolated and/or without family. In terms of the analysis of the capacity to mobilize one's own resources and those of the community, the sample presents a high number (68\%) of patients whose families could not offer their own or community resources, so they required professional help.

Related to those patients without a family, 74\% of the patients are without capacity or choose not to mobilize their own or external resources, making the intervention more difficult.

A comparison of the next group (related to the support of the family or its absence) highlights that only $25 \%$ families have the capacity to support the patient, the rest have problems in doing so $(75 \%)$. In the cases of individuals without family, the majority does not have a network of friends or else does not want their help.

In terms of the financial income of the sample, most people have low incomes, and a high number of patients do not have economic income.

Finally, although most patients have adequate housing $(55.4 \%)$, an important percentage (22\%) lives on the street or in poor housing conditions.

The analysis of the data related to the health of the sample reveals a predominance of chronic disease (linked to the diagnosis of psychosis) but that does not affect functionality, since psychiatric patients have a high functionality in daily life activities.

2.- Analysis of the association between the hospital stay and the complexity of the intervention and the association between complexity in patients with family support compared to patients without family support or social ties.

The variable duration of stay was analyzed in relation to possible associated variables, which, according to psychology literature consulted, include age, diagnosis and social complexity. Analysis was carried out using statistical techniques (ANOVA).

Quantitative variables were analyzed first using a Pearson correlation, the duration (days) and the total ECISACH-BCN PSMAR Scale score, more, the age and the duration of the days.

The correlation of days of stay and complexity, was not statistically significant (0.076), however it does suggest a trend.
Thus, we carried out a univariate analysis to know whether there is a relationship between the days of stay and the diagnosis. The F value was high, showing a relationship between the two variables.

We carried out a multiple regression analysis, which showed that intervention complexity is the variable that is statistically significant related to duration of stay, validating the first hypothesis. There is a relationship between the complexity of the intervention and the hospital stay.

To carry out the analysis of the second hypothesis (the family is a protective factor related to the complexity of social intervention), a univariate analysis (Student's t-distribution) was performed between the presence of family support and complexity. The analysis of the two variables shows that the average for patients without families is higher than the average for patients with families and that it is statistically significant, $(\mathrm{m}=39.30 \mathrm{sd} .8 .019 \mathrm{vs} \mathrm{m}=31.87 \mathrm{sd} .9 .131$; $\mathrm{t}=3.4430, \mathrm{p}<0.001)$. Thus, the second hypothesis is also verified.

\section{Conclusions}

The results obtained in this study coincide with studies carried out in other health-related fields, where there is evidence that social relations influences health in adults.

The contributions of this study lie in how these determinants may or may not influence the complexity of medical social work interventions, in terms of the importance of family and social support and the influence on the time of the hospital stay.

\section{Proposals for intervention and new lines of research}

1- Carry out the intervention protocol by medical social workers in people without family support when they require a hospital intervention in psychiatry.

2- Implement the use of the ECISACH-BCN PSMAR Scale widely in hospitalization processes.

Because of the results obtained, a line of research was created to continue to study the relationship between complexity and hospital stay. Its objective is to support the presence of medical social workers in the clinical services that have the highest complexity scores.

A new study with the department of internal medicine will begin soon, and we hope that we can implement the use of the ECISACH-BCN PSMAR Scale as an instrument for use by generalists in the hospital setting. 


\section{Introducción}

En las últimas décadas uno de los objetivos de las políticas sanitarias es determinar la importancia de los factores sociales en la salud y como estos influyen en la gestión y en la planificación de los recursos sanitarios (Lalonde,1981). Ello ha significado un cambio en la concepción del binomio salud-enfermedad, superando las teorías lineales para adoptar modelos basados en la comprensión de las realidades sanitarias de forma compleja, integral, transversal y holística.

El trabajador/a social sanitario/a (a partir de ahora TSS) es pues una pieza clave en las instituciones sanitarias que contemplan la salud de manera integral, ya que es el profesional experto en el estudio y diagnóstico de los factores sociales que afectan a la salud de las personas en los centros sanitarios. La información que proporcionan los/as TSS es fundamental para la gestión y optimización de los recursos de las instituciones sanitarias (Colom, 2012).

Los sistemas de costes intentan dar fórmulas para la gestión de los recursos sanitarios y sociales, pero es necesario alimentar estas bases diagnósticas con los factores que inciden en la complejidad de la realidad de los pacientes y como éstas influyen en su recuperación. Es por lo tanto un reto crear instrumentos que nos ayuden a cuantificar como los factores sociales inciden en la salud de las personas.

En el año 2012, el Departamento de Trabajo Social del Hospital de Lisboa Central creó la Escala ECISACH, traducida y validada a nuestro medio por el equipo de trabajo social del PSMAR (Morro, González, Pineda, Cañete, Casals, Vallvé, Campos, Conti, Moreno, Comas y Prats, 2017). Esta escala posibilita objetivar y cuantificar la afectación de estos factores en la intervención social en el marco de procesos de hospitalización, siendo un instrumento valioso tanto para el trabajador social sanitario como para el gestor hospitalario para la obtención de información sobre su ejercicio profesional y sobre la realidad con la que trabaja.

El objetivo de este estudio es describir los factores sanitarios, sociales y familiares que determinan la complejidad de la intervención social mediante la aplicación de la Escala ECISACH-BCN PSMAR en el área de hospitalización del servicio de psiquiatría del Hospital del Mar de Barcelona.

\section{La salud y su relación con los determinantes sociales}

A lo largo de la historia, el concepto de salud ha ido cambiando de significado, y si bien en un principio era un término antagónico al concepto de enfermedad, desde mediados de siglo XX esta concepción se amplia, entendiendo la salud como un completo bienestar físico, mental y social. 
En 1974, se publica en Canadá el informe Lalonde donde se definen los determinantes de la salud y como los factores sociales influyen en la mejora o en el empeoramiento de la salud de la población (Lamata,1994). Este cambio de percepción significa un cambio también en las políticas sanitarias, económicas y sociales de los países, que empiezan a estudiar cómo afectan los determinantes sociales en la vida y salud de las personas.

La Organización Mundial de la Salud (OMS) define los determinantes sociales de la salud como las circunstancias en que las personas nacen, crecen, viven, trabajan y envejecen, incluido el sistema de salud (OMS, 2007). A partir del modelo explicativo de Lalonde, se construyen diferentes teorías que han tratado de explicar cómo estos determinantes afectan a la salud de las personas (Caballero, Moreno, Sosa, Mitchel, Vega, Columbié, 2012). Diderichsen y la estratificación social (1998), el propio modelo holístico de LaframboiseLalonde (1973), Wilkinson y Marmot (1999) con su aporte desde las políticas públicas, y el más citado, el modelo de influencias en capas de Dahlgren y Whitehead (1991).

Este modelo dispone los determinantes sociales de la salud en diferentes capas superpuestas, representándolos según su proximidad o distancia a los individuos y sus familias. Como muestra la imagen, en el centro sitúa la persona y los factores de carácter individual que se relacionan con su salud (los factores individuales); en la siguiente capa los modos de vida, y en la capa más externa los factores estructurales y políticos (que inciden sobre las inequidades en la salud) y que se relacionan con la economía, las condiciones sociales y las ambientales.

\section{Gráfico 1: MODELO DE DAHLGREN Y WHITEHEAD (1991)}

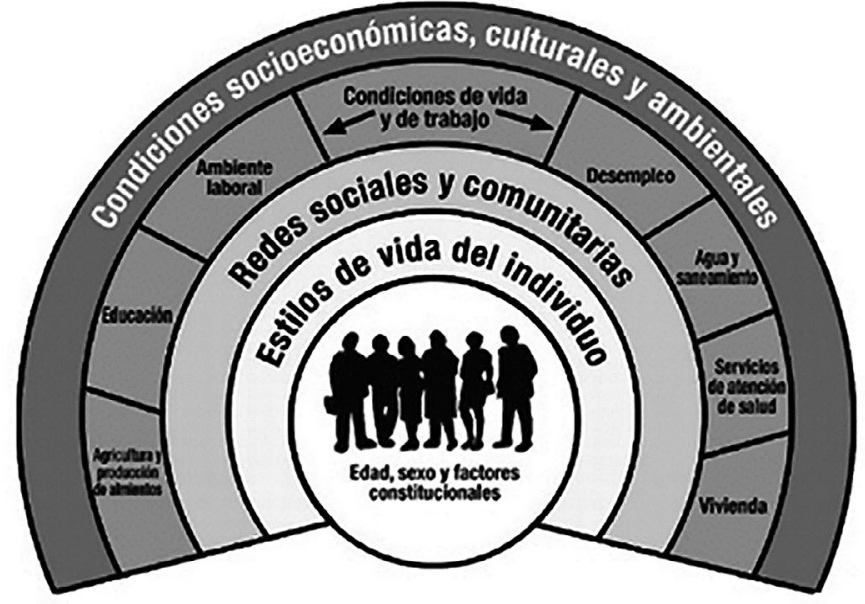

Alternativas. Cuadernos de Trabajo Social, 24, 2017, pp. 137-160 - ISSN 1133-0473 DOI: 10.14198/ALTERN2017.24.08 
En España estas teorías son desarrolladas por la Comisión para reducir las desigualdades en la Salud con el cometido de elaborar una propuesta de medidas de intervención para disminuir estas desigualdades.

\section{Gráfico 2: MARCO CONCEPTUAL DE LOS DETERMINANTES DE LAS DESIGUALDADES SOCIALES EN SALUD. (2010)}

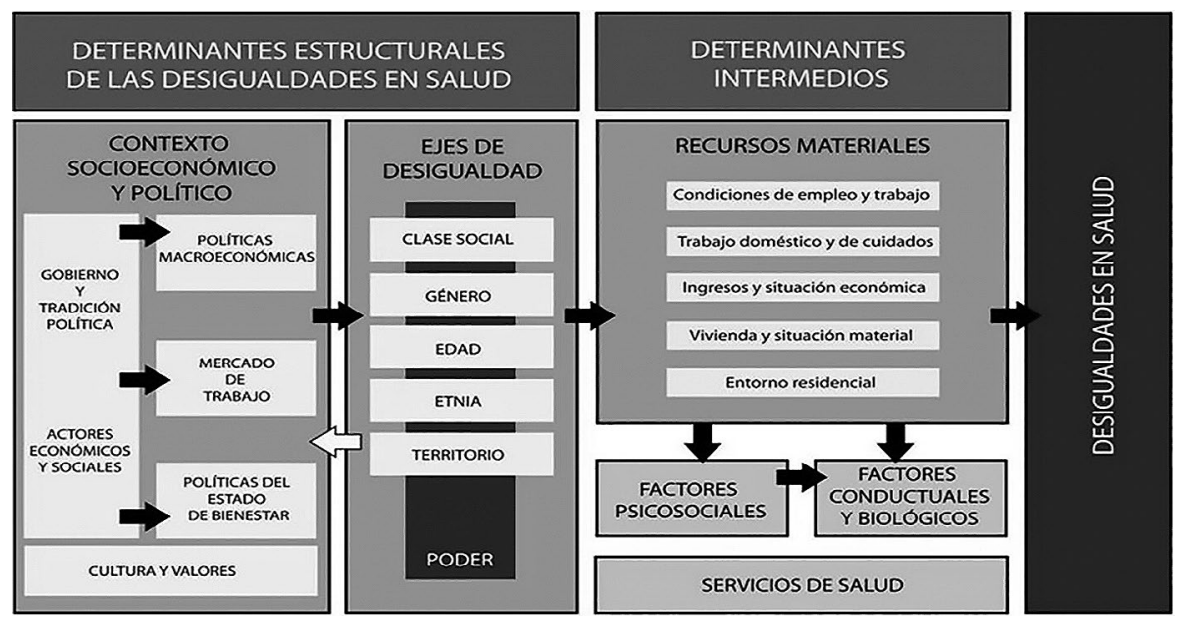

Fuente: Comisión para Reducir las Desigualdades Sociales en Salud en España, 2010

El modelo adoptado por el estado español estructura los factores intervinientes en tres categorías:

1. Contexto socioeconómico y político: la descripción de la distribución de poder y de recursos.

2. Estructura social (incluye los ejes de desigualdad que determinan las jerarquías de poder en la sociedad)

3. Los determinantes intermedios: recursos materiales más los procesos psicosociales y de conducta individual.

Por lo tanto, para establecer políticas de salud, se deben tener en cuenta las posibles patologías de la población (presentes y futuras) así como el impacto de los problemas económicos y sociales, el medio ambiente, las desigualdades, y la cultura de la sociedad.

Todos estos modelos han servido de base para la configuración de un modelo de salud holístico e integral, donde la biología y la genética comparten protagonismo con la situación económica, las redes de apoyo social, el empleo 
y condiciones de trabajo, el entorno psicosocial y demográfico, los estilos de vida, el género y la exclusión social.

\section{Salud mental y determinantes sociales}

Si estos modelos los traspasamos al ámbito de la salud mental y al tratamiento de la misma, podemos decir que la salud mental es algo más que la ausencia de trastorno, es parte integral de la salud, tanto es así que como consideramos que no hay salud sin una buena salud mental (OMS, 2012). Se calcula que una de cada cuatro personas desarrollará un problema de salud mental a lo largo de su vida, siendo más de 400 millones de personas las que experimentan una enfermedad mental en un año. Estas cifras van en aumento, y se prevé que para el año 2020 las enfermedades mentales a nivel mundial serán una de las principales causas de discapacidad.

Esta discapacidad tiene grandes costes sociales y económicos, ya que hay que sumar a los costes sanitarios (tasas muy elevadas de morbilidad y mortalidad), los costes por el desempleo, la falta de productividad, y la cronicidad de quienes la padecen y sus familiares. Si bien no hay una definición única de enfermedad mental, el manual diagnóstico de la Asociación Psiquiátrica Americana (APA), el DSM-5, define los trastornos mentales como los síndromes caracterizados por una alteración clínicamente significativa del estado cognitivo, la regulación emocional o el comportamiento de un individuo, que refleja una disfunción de los procesos psicológicos, biológicos o del desarrollo que subyacen en su función mental (Medrano,2014).

Estos síndromes se caracterizan por alteraciones importantes de la facultad de pensar y de controlar las emociones y la conducta, con una disminución de las capacidades que desarrollan las habilidades sociales y de relación. Los más graves, los llamados trastornos mentales severos (TMS) evolucionaran de forma crónica, con importantes implicaciones personales, pero también familiares y sociales.

Cuando un miembro de una familia tiene dificultades mentales, toda la familia se ve implicada, y debe adaptarse a las nuevas necesidades de su miembro enfermo. Una de las finalidades de las familias es la de cubrir las necesidades básicas de sus miembros, con la finalidad de la socialización de sus miembros en una entidad más amplia, la sociedad donde está establecida (Escartín, 1992).

Diversos estudios relacionan familia y enfermedad crónica, ya que el soporte y el apoyo familiar puede influir en el curso de la enfermedad, tanto de forma negativa como positiva (Ponce, 2007) El afrontamiento que realice la familia delante de la enfermedad de sus miembros determinará en una gran 
parte, el rol que asuma la persona enferma, la conducta de enfermedad. Este término, acuñado por Mechanic y Pilowsky (Galán, Blanco y Pérez, 2000) explica el resultado de la interacción entre variables personales y sociales en el ámbito del enfermar, y aunque es un término en desuso (implicaciones de pasividad del paciente eximido de responsabilidad en sus tratamientos) nos indica la importancia de los determinantes sociales en la salud de las personas.

Es por lo tanto importante en la planificación de estrategias de atención a la salud mental contar con líneas de apoyo y soporte a los enfermos, pero también a las familias y al soporte que la sociedad deberá brindarles. Las políticas sanitarias de atención a la salud mental deben tener en cuenta, por lo tanto, los factores que van a influir en la previsión de las enfermedades mentales, factores biológicos, pero también sociales, laborales, económicos y culturales. Estas políticas de atención a la salud mental de la población han evolucionado en el último siglo, de la mano de la investigación y de la aplicación de nuevas metodologías de investigación en las neurociencias.

La psiquiatría es una de las especialidades médicas que más ha tenido en cuenta la influencia de los factores familiares y sociales, y como estos influyen positiva o negativamente en el individuo, existiendo una voluntad de integrar los distintos elementos biopsicosociales que condicionan la patología psíquica (Vallejo,2002).

La consideración de estos factores por parte de la psiquiatría también ha condicionado los profesionales que conforman los equipos que tratan las enfermedades mentales. Estos son equipos multidisciplinares, donde en la mayoría de los casos nos encontramos con la presencia de profesionales del trabajo social, pudiendo decir que es una de las primeras especialidades médicas en incorporar a TSS en sus equipos.

\section{Trabajo social sanitario en el ámbito de la salud mental}

El TSS es la especialidad del Trabajo Social que se desarrolla y se ejerce dentro del sistema sanitario (atención primaria, la atención socio sanitaria o la atención especializada) y cuyo objetivo es de facilitar una buena salud, prevenir la enfermedad y ayudar a los enfermos y a sus familias a resolver los problemas sociales y psicosociales relacionados con la enfermedad (Barker,1991).

Si recordamos los inicios del trabajo social sanitario, con los esfuerzos del Dr. Cabot (Massachussets 1903-1905) por incorporar a las trabajadoras sociales en sus equipos, también debemos recordar que al unísono, el Dr. Sotuer y el Dr. Meyer pocos años después (1904-1913) hacían lo mismo en las instituciones psiquiátricas de la misma ciudad, junto a Mary C. Garret, una de las primeras trabajadoras sociales del área psiquiátrica, tratando de dar una respuesta 
socializadora a la cronicidad de los trastornos mentales y a la masificación de los manicomios (Garcés, 2010). Y si bien, en un principio el trabajo social tenía un marcado acento católico-asistencialista, en los años 70 evoluciona como disciplina de la mano de las nuevas corrientes de la psiquiatría (movimiento de reforma inspirados en los modelos italianos de la anti psiquiatría de Basaglia), transformándose en agente de cambio y generador de derechos de los enfermos mentales.

Actualmente los TSS forman parte de los equipos de atención de salud mental, y podemos vertebrar sus funciones en varios ejes (atención directa, e indirecta, coordinación, gestión del caso, etc.), pero con un objetivo principal: la elaboración del diagnóstico social sanitario, con el que planificará su intervención y la del equipo. También se ejercen funciones de planificación y promoción de la salud del individuo y su comunidad, así como acciones de formación docencia e investigación. Y si bien, la Ley 44/2003, de 21 de noviembre de Ordenación de las Profesiones Sanitarias, no incluye entre los profesionales sanitarios al trabajador social, en la práctica, los TSS se encuentran presentes en los dispositivos de salud mental, desde la atención primaria hasta la hospitalización, pasando por los servicios de rehabilitación, con el objetivo de impulsar, fortalecer y rescatar los aspectos más sanos de los pacientes y de sus familias, mediante el trabajo social clínico (Ituarte, 2017), aportando el diagnostico social sanitario al plan de intervención integral que realizan todos los miembros del equipo multidisciplinar (Colom, 2012).

\section{Diagnóstico social sanitario: la intervención del TSS desde la perspectiva de las teorías de la complejidad}

El entorno en el que debe intervenir el TSS en el área de Psiquiatría es complejo y diverso, donde se interrelacionan los determinantes de la salud, los factores personales, familiares y sociales de la persona y la enfermedad mental. Por ello es necesario aplicar un marco teórico que contemple esta transversalidad y complejidad de los hechos, que acepte la coexistencia de las diferentes posiciones de la persona enferma, de su familia y su entorno, para poder realizar un diagnóstico social sanitario ajustado a su realidad.

Edgar Morin, sociólogo francés, nos ofrece esta posibilidad de mirar de otra forma las realidades sociales mediante el Paradigma de la Complejidad, modelo teórico presentado en su obra El Método. En esta obra Morin expone que no hay realidades excluyentes, sino que la realidad se compone de sistemas totales complementarios y contradictorios, que no pueden abarcarse ni comprenderse si no es asumiendo su complejidad intrínseca, donde el individuo no se entiende sino es en relación con otros y con su entorno. 
Este autor define (los principios básicos del pensamiento complejo y que podemos aplicar a la comprensión de la realidad sanitaria (Morin, 1994):

1. El principio sistémico u organizacional: donde se relaciona las partes con el todo.

2. El principio Holo gramático: las partes están en el todo, y el todo en las partes

3. El principio reactivo: la causa actúa sobre el efecto y el efecto sobre la causa

4. El principio recursivo: la auto organización y autoproducción.

5. El principio de autonomía y dependencia del medio

6. El principio dialógico, que integra lo antagónico como complementario

7. Y por último el de reintroducción del sujeto como creador del pensamiento.

Esta visión antroposocial, donde se incluye las dimensiones de las ciencias naturales y las ciencias sociales supone una construcción teórica muy rica para el TSS, que se nutre de ambas ciencias para construir su conocimiento (Cordero y Blanco, 2004). Podríamos decir que está en el ADN de los TSS esta visión de la complejidad que nos propone Morin, ya que los TSS son los profesionales del equipo que aportan mediante el diagnostico social sanitario esta visión compleja de la realidad de la persona enferma.

El diagnostico social sanitario contempla a la persona íntegramente, desde su «yo» (personalidad) pasando por su entorno más cercano (familia), su entorno (medio, contexto donde vive) y la enfermedad, considerando ésta como un factor disruptivo que va a cambiar todas las dinámicas internas y externas (su psique, sus relaciones familiares, laborales y sociales) y que determinarán su futuro (Colom, 2012). Para realizar el estudio diagnostico desde la disciplina del TSS se utilizan diferentes herramientas, entre las más relevantes: la entrevista clínica (ya sea al paciente y/o a su familia o personas de su entorno), la observación, las encuestas, y las escalas.

Las escalas son instrumentos científicos que sirven para medir y representar el estado de una o unas variables en un momento determinado. El Trabajo Social tiene diferentes escalas que miden diferentes variables, como por ejemplo el riesgo social, la exclusión social, la funcionalidad, la claudicación familiar, el apoyo social, etc. y que nos sirven de base para por un lado conocer situaciones concretas, y por otro poder compararlas de una manera objetiva con otras situaciones para llevar a cabo una teorización deductiva generando un conocimiento abstracto basado en la práctica clínica. 


\section{La escala ECISACH-BCN PSMAR}

En el ámbito del TSS no se disponía de un instrumento que midiera la complejidad de la intervención social hasta que en 2012, un grupo de trabajadoras sociales sanitarias del Hospital Central de Lisboa (Sefarim,2012) aplicaron las teorías descritas por Edgar Morin (el paradigma de la complejidad) a la intervención desde el TSS, creando un instrumento que identifica la complejidad de un proceso de intervención según una situación social concreta, la Escala de Complejidad de la Intervención Social con Adultos en un Contexto Hospitalario, en adelante Escala ECISACH. Esta escala portuguesa es utilizada en los centros hospitalarios de Portugal, y es un instrumento de calidad de las diferentes instituciones que lo aplican. Se ha traducido y validado esta escala en el contexto español con el nombre de ECISACH-BCN PSMAR (Morro, González, Pineda, Cañete, Casals, Vallvé, Campos, Conti, Moreno, Comas y Prats, 2017)

\section{Objetivo del estudio}

Describir los factores que determinan la complejidad de la intervención social de los pacientes ingresados en una unidad de psiquiatría hospitalaria mediante la aplicación de la Escala ECISACH-BCN PSMAR.

\section{Objetivo principal}

Medir la complejidad de la intervención social en la hospitalización psiquiátrica investigando que factores de la enfermedad mental y de su contexto familiar y social determinan su gravedad.

\section{Objetivos secundarios}

- Describir la realidad familiar y social de los pacientes ingresados en Psiquiatría.

- Aplicar la Escala ECISACH-BCN PSMAR en el ámbito de la psiquiatría

- Explorar si la información que proporciona la Escala es un buen instrumento para la gestión hospitalaria.

\section{Hipótesis}

Las hipótesis de tipo correlacional que se han elaborado durante el estudio son:

I. Existe una relación entre la complejidad de la intervención y la duración de la estancia hospitalaria 
II. La familia es un factor protector frente a la complejidad de la intervención social: los pacientes con familia presentan menor complejidad de la intervención social en los ingresos hospitalarios y al revés, los pacientes sin familia presentan mayor complejidad.

\section{Metodología: Diseño del estudio}

Se trata de un estudio descriptivo correlacional, de carácter transversal, que estudia las variables en un espacio de tiempo corto (el ingreso en la unidad psiquiátrica de agudos), y que tiene en cuenta la relación entre los resultados de la escala ECISAH-BCN PSMAR con las variables socio-demográficas y clínicas de la muestra.

Los estudios correlacionales determinan si dos variables están correlacionadas o no, analizando si un aumento o disminución en una variable coincide con un aumento o disminución en la otra variable (Hernández, 2007). Primero se miden las variables y luego, mediante la aplicación de técnicas estadísticas, se estima si hay correlación o no. Si bien es un tipo de estudio que no establece de forma directa relaciones causales, aporta posibles causas de las mismas.

Hay diferentes tipos de correlaciones: las positivas, las negativas y la no correlación. Esta investigación se ha centrado en las positivas, que pueden aportar una mayor información a las hipótesis.

El estudio se ha realizado en el servicio de Psiquiatría del Hospital del Mar de Barcelona. Este servicio de agudos consta de dos unidades hospitalarias: la unidad 07 (con 7 camas) y la unidad 80 (35 camas).

La población que constituye la muestra del estudio está formada por aquellos pacientes que ingresaron en las unidades de agudos de Psiquiatría del Hospital del Mar durante el periodo comprendido de enero a mayo de 2017 y que han requerido de intervención social por parte del TSS, con una participación final de 83 personas. ( $\mathrm{N}=83$ )

Los datos de la muestra se recogen durante el proceso de intervención social que realiza el TSS durante el tiempo de ingreso del paciente. Tras el alta del paciente de la unidad de Psiquiatría se dispone de la puntuación total de la escala ECISACH-BCN PSMAR, que se completa al finalizar el ingreso hospitalario.

Esta escala consta de 12 ítems agrupados en tres subescalas o variables:

- la primera es el marco sociofamiliar del paciente analizándose el vínculo familiar, la capacidad de movilización de recursos, la disponibilidad de apoyo, los ingresos y la vivienda.

- la segunda variable la situación clínica, que analiza el estado clínico del paciente y su autonomía. 
- la tercera, analiza el proceso de intervención social, contabilizando el número de atenciones, de contactos o coordinaciones, la disponibilidad de apoyo y el tiempo de resolución del caso.

Cada ítem se evalúa con una escala numérica del 1 al 5 siendo el 1 la situación ideal y el 5 la situación más compleja.

La puntuación total se obtiene de la suma de las puntuaciones de las subescalas con unas puntuaciones repartidas en cinco tramos que describen de menor a mayor la complejidad de la intervención: sin complejidad, complejidad baja, complejidad intermedia, alta complejidad y complejidad muy alta.

Todos estos datos se compilan en una base de datos que consta de ítems de carácter sociodemográficos (edad, género, fecha de ingreso y de alta), datos clínicos (diagnóstico psiquiátrico expresado en CIE-9) y los doce ítems relacionados con ECISACH-BCN PSMAR.

Se ha realizado un análisis estadístico mediante el programa estadístico SPSS (versión 24.0), tanto descriptivo como analítico univariante (correlación de Pearson, t-student y ANOVA y multivariante (regresión múltiple) para testar las hipótesis a estudio.

\section{Resultados}

Durante el periodo de estudio, de enero a mayo del 2017, se ha intervenido a nivel social en 109 casos. Esto significa un $43.4 \%$ del total de ingresos de psiquiatría (con una N. total de 251).

De los 109 casos, sólo se ha podido incluir en el estudio 83, ya que los 26 restantes se encontraban aun en procesos activos de hospitalización en el momento del análisis estadístico. Esto significó la imposibilidad de obtener las variables correspondientes a la Escala ECISACH-BCN PSMAR (que se suman al alta hospitalaria) así como los ítems de destino al alta y duración del ingreso, por lo que estos casos han tenido que ser descartados.

La presentación de los resultados se vertebra en dos ejes:

1. Análisis descriptivo de las variables cualitativas y cuantitativas sociodemográficas clínicas y de puntuación total de la escala ECISACH-BCN PSMAR.

2. Análisis descriptivo de las subescalas de la Escala ECISACH-BCN PSMAR.

3. Comparación entre estancia hospitalaria, complejidad social y apoyo familiar. 


\section{Descripción de las variables cualitativas y cuantitativas}

Para el análisis cuantitativo de los datos se han utilizado índices de estadística descriptiva como la media, la desviación estándar para las variables cuantitativas (edad, días de ingreso y la puntuación total de la Escala ECISACH) y los porcentajes en las variables cualitativas: sexo, diagnostico, nacionalidad, derivación al alta.

En la tabla 1 se muestra la media y desviación estándar de las variables cuantitativas: la edad, los días de ingreso y la puntuación total de la Escalad ECISACH-BCN PSMAR.

En cuanto a la edad, la cifra apunta a edad bastante inferior a las muestras de hospitalización general (diferentes estudios, como el Estudio Epine, sitúan la edad media en hospitalización por encima de los sesenta años)

También cabe destacar que la duración de los ingresos en el servicio es extensa, con 24 días de media (la estancia Gold Estándar en Psiquiatría es de 16 días).

La media de la puntuación total de complejidad en la intervención social mediante la escala ECISACH se ha situado en el rango de la complejidad intermedia, y tratándose de casos donde se ha solicitado la intervención de la TSS no es muy alto.

Tabla 1: DESCRIPCIÓN DE VARIABLES CUANTITATIVAS

\begin{tabular}{|l|l|l|l|}
\hline & N & Media & Desviación Estándar \\
\hline Edad & 83 & $\mathbf{4 8 , 2 0}$ & 19,366 \\
\hline Días ingreso & 83 & $\mathbf{2 4 , 6 0}$ & 13,956 \\
\hline $\begin{array}{l}\text { Puntuación total ECISACH_BCN } \\
\text { PSMAR }\end{array}$ & 83 & $\mathbf{3 3 , 5 2}$ & 10,055 \\
\hline N Valida & 83 & & \\
\hline
\end{tabular}

Respecto a las variables cualitativas, hay un predominio muy ligero de hombres (53\%), hecho común en las unidades de atención psiquiátrica. En referencia a los diagnósticos, ante la diversidad de los diagnósticos hallados en la muestra (codificados en CIE9), se ha realizado una reducción diagnóstica, agrupando los trastornos psicóticos y bipolares con el modelo de psicosis única (Peralta, Basterra, Zandio y Cuesta,2008).

Los resultados nos indican un claro predominio de los trastornos psicóticos en los ingresos en la unidad de agudos ( $88 \%$ de los ingresados). En cuanto a las 
derivaciones al alta, hay un alto porcentaje de pacientes (67\%) que requieren de prolongar su estancia hospitalaria en un recurso de media estancia (subagudos) por la necesidad de estabilización clínica, frente al $43 \%$ de pacientes que son dados de alta al domicilio.

\section{Análisis descriptivo de las subescalas de la escala ECISACH-BCN PSMAR}

Como ya se ha comentado anteriormente, la Escala ECISACH-BCN PSMAR consta de 12 ítems agrupados en tres subescalas: marco sociofamiliar, salud e intervención social. A continuación, se presenta el análisis descriptivo de estos ítems, que proporcionan una información muy valiosa de la muestra (tanto a nivel social como de gestión de recursos).

Marco socio-familiar: En cuanto a las relaciones familiares, si bien un $36.1 \%$ de los pacientes mantiene una buena relación familiar, el $41 \%$ de los pacientes presenta distocia familiar, siendo un alto porcentaje, casi un $23 \%$, los pacientes aislados y/o sin familia. Al analizar la capacidad de estas familias para movilizar sus recursos y los de la comunidad, la muestra presenta un número alto (68\%) de pacientes cuyas familias no pueden ofrecerles recursos propios o de la comunidad, por lo que requieren de ayuda profesional.

Si nos fijamos en el grupo de pacientes sin familia, los porcentajes empeoran, encontrándonos con un $74 \%$ de los pacientes sin capacidad o con rechazo para la movilización de recursos propios o externos, lo que dificultará la intervención.

Al comparar el siguiente grupo (relacionado con el apoyo de la familia o su ausencia), resalta que sólo en un $25 \%$ de los casos las familias tienen capacidad para apoyar al paciente, presentando problemas el resto (75\%).

En los casos de individuos sin familia, la mayoría no tienen red de amigos o rechazan su ayuda (60.9\%).

Cuando se examinan los datos de ingresos económicos de la muestra, se constata que se trata de personas con rentas bajas, y con un alto número de pacientes sin ingresos económicos (70\% de la muestra percibe menos que el salario mínimo interprofesional).

Por último, si bien la mayoría de los pacientes tiene una vivienda adecuada (55.4\%) un porcentaje importante (22\%) vive en la calle o en condiciones de ruina o barraquismo.

Situación clínica: La escala dispone de dos ítems que valoran la frecuencia, duración y grado de discapacidad de la enfermedad en el paciente. Se trata de una adaptación de la escala FIM (o MIF en portugués), escala de independencia 
funcional, que estima los niveles de discapacidad cualquiera que sea su origen o naturaleza.

$\mathrm{Al}$ analizar los datos relativos a la salud de la muestra, hay un predominio de la enfermedad crónica $(55,4 \%)$ pero que no afecta a la funcionalidad, ya que los pacientes psiquiátricos tienen un porcentaje de funcionalidad sobre las actividades de la vida diaria (AVD) bastante alto. (44,6\%)

Trabajo Social Clínico: Los ítems referentes al TSS son los referentes a la intervención, al tiempo y a los recursos (y su disponibilidad).

Son aspectos importantes en las hospitalizaciones y que marcarán la planificación del alta hospitalaria, así como aportarán información sobre la complejidad de nuestra intervención.

La escala discrimina entre: la intervención, el número de asistencias, el número de contactos o coordinaciones, la disponibilidad de las respuestas y el tiempo de resolución social.

En la tabla 2 podemos observar los diferentes porcentajes referentes a la actividad de la intervención social que se clasifican en la Escala ECISACHBCN PSMAR.

Tabla 2: INTERVENCIÓN SOCIAL

\begin{tabular}{|l|c|c|}
\hline & Frecuencia & $\begin{array}{c}\text { Porcentaje } \\
\text { Valido }\end{array}$ \\
\hline Información /orientación/acompañamiento psicosocial & 15 & 18,1 \\
\hline Asesoramiento social /información & 12 & 14,5 \\
\hline $\begin{array}{l}\text { Información/orientación, acompañamiento psicosocial, } \\
\text { derivación a un equipamiento/devolución social o } \\
\text { elaboración de un informe social interno o externo }\end{array}$ & 40 & 48,2 \\
\hline $\begin{array}{l}\text { Información/orientación, acompañamiento psicosocial, } \\
\text { derivación hasta dos equipamientos/devolución social } \\
\text { o elaboración de un informe social interno o externo } \\
\text { y/o realización de una gestión fuera de la institución } \\
\text { (visita domiciliaria / acompañamiento a gestiones } \\
\text { administrativas/reuniones externas) }\end{array}$ & 12 & 14,5 \\
\hline $\begin{array}{l}\text { Información/orientación, acompañamiento psicosocial, } \\
\text { derivación a más de dos equipamientos/devolución social } \\
\text { o elaboración de un informe social interno o externo } \\
\text { y/o realización de una gestión fuera de la institución } \\
\text { (visita domiciliaria / acompañamiento a gestiones } \\
\text { administrativas/reuniones externas }\end{array}$ & 4 & 4,8 \\
\hline \begin{tabular}{l} 
Total \\
\hline
\end{tabular} & 83 & 100,0 \\
\hline
\end{tabular}


En cuanto al número de asistencias, las atenciones se sitúan en la franja inicial de la tabla, con dos atenciones en un $41 \%$ de los casos, posiblemente explicable por ser un centro de agudos, de ingresos breves, donde las atenciones sociales disminuyen, potenciándose las de otros profesionales. De la misma manera, el número de contactos con la comunidad se sitúa en la misma franja, situándose entre los dos y cuatro contactos o coordinaciones (42.1\%).

En cambio, el ítem se mide la disponibilidad de las respuestas institucionales, relevante para la planificación del alta hospitalaria, que hay dificultades graves en estas, no habiendo el recurso necesario, adecuado para el paciente psiquiátrico en el ámbito de lo social (sobre todo en el ámbito residencial).

Esta falta de respuesta de la comunidad para cubrir las necesidades sociales hace que el tiempo de resolución social se alargue, situándose en la franja de más de 15 días la resolución del caso, alargándose también la estancia hospitalaria.

Como en los dos anteriores ítems hay una cierta polaridad con el ítem menos complejo, seguramente correspondiente a los pacientes con buen soporte o familia ( $25,3 \%$ frente al $32,5 \%$ ).

Tras la descripción de los ítems de la escala, confrontaremos las hipótesis planteadas al inicio del estudio con los datos estadísticos del estudio, mediante la asociación de las diferentes variables implicadas.

\section{Comparación entre estancia hospitalaria, complejidad social y apoyo familiar}

Tras la descripción de los ítems de la escala, confrontaremos las hipótesis planteadas al inicio del estudio con los datos estadísticos del estudio, mediante la asociación de las diferentes variables implicadas.

\section{Hipótesis I}

Existe una relación entre la complejidad de la intervención y la duración de la estancia hospitalaria

Para demostrar esta hipótesis, en primer lugar, hemos realizado un análisis univariante entre la duración del ingreso con las posibles variables asociadas, que, según la literatura consultada (Seva-Diaz y Seva-Fernández, 2003) en el ámbito de la psiquiatría son: la edad, el diagnóstico y la complejidad social, utilizando las técnicas estadísticas adecuadas (ANOVA y correlaciones).

Tras ese análisis se utilizaron técnicas de regresión para determinar qué factores afectan independientemente la variable de estudio (duración del ingreso) 
Para ello hemos analizado en primer lugar dos variables cuantitativas mediante una correlación de Pearson, la duración (días) y el total de la Escala ECISACH, y a continuación dos variables más, la edad y la duración de los días.

Tabla 3: CORRELACIÓN DE PEARSON DE LAS VARIABLES EDAD, DURACIÓN DEL INGRESO Y DEL TOTAL DE LA ESCALA ECISACH-BCN PSMAR

\begin{tabular}{|c|c|c|c|}
\hline & & DÍAS & EDAD \\
\hline \multirow{4}{*}{ DÍAS } & Correlación Pearson & 1 &,$- 235^{*}$ \\
\hline & Sig. (bilateral) & & ,032 \\
\hline & $\mathrm{N}$ & 83 & 83 \\
\hline & Correlación Pearson &,$- 235^{*}$ & 1 \\
\hline \multirow[t]{3}{*}{ EDAD } & Sig. (bilateral)* & ,032 & \\
\hline & $\mathrm{N}$ & 83 & 83 \\
\hline & & dias & TOTAL \\
\hline \multirow{4}{*}{ DÍAS } & Correlación Pearson & 1 & , 196 \\
\hline & Sig. (bilateral) & & ,076 \\
\hline & $\mathrm{N}$ & 83 & 83 \\
\hline & Correlación Pearson & ,196 & 1 \\
\hline \multirow[t]{2}{*}{ TOTAL } & Sig. (bilateral)* & ,076 & \\
\hline & $\mathrm{N}$ & 83 & 83 \\
\hline
\end{tabular}

* La correlación es significativa al nivel 0,05 (bilateral)

El resultado en el caso de correlación días de ingreso-complejidad, si bien no ha sido significativo estadísticamente (0'076) si sugiere una tendencia, y dado nuestra hipótesis a priori hemos decidido realizar un multivariante para descartar factores de confusión.

A continuación, realizamos una ANOVA univariante para conocer si hay relación entre los días de ingreso (variable dependiente cuantitativa) y los diagnósticos (variable independiente categórica). El valor de F es alto, por lo que significa que estas dos variables están relacionadas (tabla 4). 
Tabla 4: ANOVA

\begin{tabular}{|l|c|c|c|c|c|}
\hline \multicolumn{7}{|c|}{ ANOVA } \\
\hline & $\begin{array}{c}\text { Sumade } \\
\text { cuadrados }\end{array}$ & $g l$ & $\begin{array}{c}\text { Media } \\
\text { cuadrática }\end{array}$ & F & Sig. \\
\hline Inter-grupos & 2141,626 & 3 & 713,875 & 4,205 &, 008 \\
\hline Intra-grupos & 13412,398 & 79 & 169,777 & & \\
\hline Total & 15554,024 & 82 & & & \\
\hline
\end{tabular}

Tras este primer análisis, y viendo la posible influencia de la complejidad de la intervención en la estancia, realizamos una regresión múltiple (variables independientes diagnóstico, edad y complejidad y variable dependiente días de ingreso)

Tabla 5: MODELO DE REGRESIÓN

\begin{tabular}{|c|c|c|c|c|c|c|}
\hline \multirow{2}{*}{ Modelo } & \multicolumn{2}{|c|}{$\begin{array}{l}\text { Coeficiente no } \\
\text { estandarizado }\end{array}$} & \multirow{2}{*}{$\mathrm{t}$} & \multirow{2}{*}{ Sig. } & \multicolumn{2}{|c|}{$\begin{array}{c}\text { Intervalo de } \\
\text { Confianza para B } \\
\text { al } 95 \%\end{array}$} \\
\hline & B & $\begin{array}{l}\text { Error } \\
\text { típ. }\end{array}$ & & & $\begin{array}{l}\text { Límite } \\
\text { inferior }\end{array}$ & $\begin{array}{l}\text { Límite } \\
\text { superior }\end{array}$ \\
\hline (Constante) & $\begin{array}{c}-4,007 \mathrm{E}- \\
013 \\
\end{array}$ & 12,739 &, 000 & 1,000 & $-25,366$ & 25,366 \\
\hline EDAD &,- 139 & ,082 & $-1,703$ & ,093 &,- 301 & ,023 \\
\hline $\begin{array}{l}\text { COMPLEJIDAD DE LA } \\
\text { INTERVENCION } \\
\text { SOCIAL }\end{array}$ & ,305 & , 154 & 1,985 & ,051 &,- 001 & ,611 \\
\hline $\begin{array}{l}\text { DIAGNOSTICO } \\
\text { REDUCIDO21 }\end{array}$ & 22,824 & 14,072 & 1,622 & , 109 & $-5,197$ & 50,845 \\
\hline $\begin{array}{l}\text { DIAGNOSTICO } \\
\text { REDUCIDO31 }\end{array}$ & 8,043 & 16,844 & ,478 & ,634 & $-25,497$ & 41,584 \\
\hline $\begin{array}{l}\text { DIAGNOSTICO } \\
\text { REDUCIDO41 }\end{array}$ & 11,369 & 15,133 & ,751 & ,455 & $-18,764$ & 41,503 \\
\hline $\begin{array}{l}\text { DIAGNOSTICO } \\
\text { REDUCIDO51 }\end{array}$ & 7,290 & 18,668 & ,390 & ,697 & $-29,883$ & 44,462 \\
\hline
\end{tabular}


Los análisis regresivos consisten en estudiar la relación entre variables, para conocer si existe una asociación, cuanta fuerza tiene (coeficiente de correlación) y su forma y /o modelo.

En este caso vemos que la complejidad de la intervención es la variable que es estadísticamente significativa en relación con la estancia y su duración, por lo que podemos decir que la primera hipótesis se verifica: hay una relación entre la complejidad de la intervención y la estancia hospitalaria.

\section{Hipótesis II}

La familia es un factor protector frente a la complejidad de la intervención social: los pacientes con familia presentan menor complejidad de la intervención social en los ingresos hospitalarios y al revés, los pacientes sin familia presentan mayor complejidad Se realiza un análisis univariante (tstudent) entre la presencia de familia y la complejidad.

$\mathrm{Al}$ analizar las dos variables vemos que la media de los pacientes sin familia es superior a la media de los pacientes con familia de forma estadísticamente significativa, $(\mathrm{m}=39.30 \mathrm{sd} .8 .019 \mathrm{vs} \mathrm{m}=31.87 \mathrm{sd} .9 .131 ; \mathrm{t}=3.4430, \mathrm{p}<0.001)$, por lo que podemos decir que la hipótesis dos también se verifica.

Tabla 6: ANÁLISIS COMPLEJIDAD Y PRESENCIA DE FAMILIA

\begin{tabular}{|c|c|c|c|c|c|c|}
\hline & & $\mathrm{N}$ & MEDIA & $\begin{array}{l}\text { Desviación } \\
\text { Estándar }\end{array}$ & \multicolumn{2}{|c|}{ Std. Media Error } \\
\hline & $\begin{array}{l}\text { Pacientes sin } \\
\text { familia }\end{array}$ & 23 & 39,30 & 8,019 & \multicolumn{2}{|c|}{1,672} \\
\hline TOTAL & $\begin{array}{l}\text { Pacientes con } \\
\text { familia }\end{array}$ & 60 & 31,87 & 9,131 & \multicolumn{2}{|c|}{1,179} \\
\hline & & \multicolumn{2}{|c|}{$\begin{array}{l}\text { Test de Levene para } \\
\text { igualdad de varianzas }\end{array}$} & & & \\
\hline & & $\mathrm{F}$ & Sig. & $\mathrm{t}$ & df & $\begin{array}{c}\text { Sig. } \\
\text { (2-tailed) }\end{array}$ \\
\hline & $\begin{array}{l}\text { Igualdad de } \\
\text { varianzas } \\
\text { asumidas }\end{array}$ & 1,261 & ,265 & 3,430 & 81 & ,001 \\
\hline TOTAL & $\begin{array}{l}\text { Igualdad de } \\
\text { varianzas no } \\
\text { asumidas }\end{array}$ & & & 3,635 & 45,141 &, 001 \\
\hline
\end{tabular}




\section{Discusión}

Los resultados obtenidos en este estudio coinciden con los estudios realizados en diferentes campos de la salud, donde se evidencia que las relaciones sociales influyen en los resultados de la salud de los adultos (Holt-Lunstad, Smith y Layton, 2010): la estancia hospitalaria tiene relación con la complejidad de la intervención social y la presencia de apoyo familiar es un factor protector frente a la complejidad de la intervención social.

El apoyo social aumenta la capacidad de los pacientes para hacer frente y adaptarse a la vida diaria (Gutiérrez-Maldonado, Caqueo-Urízar, Ferrer-García y Fernández-Dávila, 2012) y si lo traspasamos al campo de la salud mental, nos encontramos que los pacientes con apoyo socio-familiar tienen mejores condiciones de vida y presentan menores ingresos hospitalarios que aquellos que carecen de este apoyo (Norman, Malla y Manchanda, 2005).

El apoyo familiar también influye en el curso de las enfermedades crónicas (como las enfermedades mentales graves), y puede convertirse en un factor de protección o de riesgo según se cuente con él o no (Ponce, López, Velázquez, Márquez, Bellido y Cruz, 2007). Por lo tanto, los diferentes estudios ya nos presentaban la influencia de estos determinantes sociales en la salud (mental) de los pacientes.

La aportación de este estudio al tema que tratamos es cómo estos determinantes influyen o no en la complejidad de la intervención del Trabajador/a Social Sanitario/a, el peso que tiene el apoyo familiar y social en el nivel de complejidad de la intervención, y si esto a su vez influye en la estancia hospitalaria. Los resultados nos indican que los pacientes con menor apoyo familiar y menor disponibilidad de relaciones sociales afectivas tienen un nivel de complejidad más alto que el resto de los pacientes.

Así mismo los análisis estadísticos nos indican que la complejidad de la intervención social y la duración del ingreso hospitalario están relacionadas, por lo que podemos decir que el apoyo familiar y social de los pacientes determinara el nivel de intervención social (complejidad) y la duración del ingreso. Esto es relevante ya que conocer estos determinantes nos permitiría desarrollar estrategias adecuadas, protocolos de intervención, sobre el colectivo que presenta una mayor complejidad en la intervención, la persona sin apoyo familiar

Ello podría significar una reducción en la estancia hospitalaria, pudiendo ser objeto de siguientes estudios. Estudiar entonces el apoyo social y familiar de los pacientes es un factor importante para predecir recursos necesarios para el tratamiento de los pacientes en el ámbito hospitalario, siendo un factor prioritario en las valoraciones psicosociales de los pacientes. La escala ECISACH-BCN PSMAR es un buen instrumento para medir y evaluar la complejidad de la 
intervención social, su utilización en el estudio ha posibilitado realizar un análisis descriptivo de los factores sociales, clínicos y característicos de la intervención social, por lo que recomendamos su incorporación a la práctica cuotidiana del profesional del Trabajo Social Sanitario.

\section{Limitaciones}

Este estudio tiene limitaciones de carácter temporal y estructural. En cuanto a la limitación temporal, el estudio se ha realizado durante 4 meses y pudiera ser que la muestra recogida no sea significativa.

La limitación estructural tiene que ver con la muestra también, ya que ésta tiene el sesgo de que ya son pacientes derivados al TSS, por lo que alguien (profesionales, la familia, o el propio usuario) ya han detectado un riesgo social.

Para poder realizar el estudio sin estigmas, el número total de personas atendidas por el TSS debería ser el número total de ingresos en la unidad, todas las personas que ingresan en la unidad deberían ser valoradas por el TSS y así poder analizar mediante la escala ECISACH-BCN PSMAR las características de nuestra intervención.

\section{Bibliografía}

BARKER, R. (1991). The Social Work Dictionary. Silver Spring, MD: National Association of Social Workers.

Caballero, G.E., Moreno, G.M., Sosa, C. M.E., Mitchel, F.E., Vega, H.M., ColumbiÉ, P. (2012). Los determinantes sociales de la salud y sus diferentes modelos explicativos. INFODIR, 8 (15).

Colom, D. (2012). El Trabajo social sanitario como herramienta de gestión. Sedisa, $14-24$.

Colom, D. (2012). El diagnóstico social sanitario. Aval de la intervención y seña de identidad del trabajo social sanitario. Barcelona: UOC.

Cordero, N. y Blanco, J. (2004). ¿Trabajo social sin fundamento? Aportaciones del paradigma de la complejidad a la epistemología del Trabajo Social. Portularia, 4, 407-412.

DAHLGReN, G. y WhiteHEAD, M. (1992). Policies and strategies to promote equity in health. Copenhagen: WHO Regional Office for Europe. EUR/ICP/RPD 414 (2).

EsCARTín, M.J. (1992). El sistema familiar y el trabajo social Alternativas: Cuadernos de trabajo social, 1, 55-75.

Galán, A., Blanco, A. Pérez, M.A. (2000). Análisis del concepto de Conducta de Enfermedad: Un acercamiento a los aspectos psicosociales del enfermar. Anales de psicología, 16, (2), 157-166. 
Garcés Trullendue, E.M. (2010). El Trabajo Social en Salud Mental. Cuadernos de Trabajo Social, 23, 333-352.

Gutiérrez, J., Caqueo, A., Ferrer, M., \& Fernández, P. (2012). Influencia de la percepción de apoyo y del funcionamiento social en la calidad de vida de pacientes con esquizofrenia y sus cuidadores. Psicothema, 24(2), 255-262.

HERNÁNDEZ R.; FERNÁNDEZ, C. y BAPTISTA, P. (2007). Metodología de la Investigación. Ciudad de México: McGraw-Hill. 6 edición.

Holt-Lunstad, J., Smith, T.B. y Layton, J.B. (2010). Social Relationships and Mortality Risk: A Meta-Analytic Review. PLoS Medicine 7 (7) https://doi. org/10.1371/journal.pmed.1000316

Ituarte Tellaeche, A. (coord.) (2017). Prácticas del trabajo social clínico. Valencia: Nau Llibres.

LALONDE, M. (1974). A new perspective on the health of Canadians. Ottawa: Minister of Supply and Services Canada. Retrieved from Public Health Agency of Canada website: http://www.phac-aspc.gc.ca/ph-sp/pdf/perspect-eng.pdf

LAmatA, F. (1994). Una perspectiva de la política sanitaria 20 años después del informe Lalonde. Gaceta Sanitaria, 8, 189-94. https://doi.org/10.1016/ S0213-9111(94)71192-4

MEDRANO,J. (2014). Boticarium. Revista de la Asociación Española de Neuropsiquiatría, 34 (121), 175-194. https://doi.org/10.4321/S0211-57352014000100011

Ministerio DE SANidAD y CONSUMO (1998). Análisis y Desarrollo de los GDR en el Sistema Nacional de Salud. Madrid: Ministerio de Sanidad y Consumo

Morín, E. (2004). Introducción al pensamiento complejo. México: Gedisa

Morro, L., González, S., Pineda, I., Cañete, M.J., Casals, A., Vallvé, M., Campos, S., Conti, M., Moreno, A., Comas, M. y Prats, A. (2017). Trabajo social sanitario y complejidad: traducción al español y validación a nuestro medio de la escala de complejidad de la intervención social con adultos en un contexto hospitalario (ECISACH), la escala ECISACH-BCN PSMAR. Revista Aghatos. Atención Socio sanitaria y Bienestar, 1, 48-54.

Norman, RMG, Malla, AK, Manchanda, R, Harricharan, R, TAKHAR, J \& NORTHCOTT, S. (2005). Social support and three-year symptom and admission outcomes for first episode psychosis. Schizophrenia Research, 80, 227-344. https://doi.org/10.1016/j.schres.2005.05.006

OMS (2007). Commission on social determinants of health. A conceptual framework for action on the social determinants of health. http://www.who.int/sdhconference/resources/ConceptualframeworkforactiononSDH_eng.pdf

OMS (2017). Plan de acción sobre la salud mental 2013-2020. http://apps.who.int/ iris/bitstream/10665/97488/1/9789243506029_spa.pdf

Peralta, V., Basterra, V., Zandio, M. y Cuesta, MJ. (2008). Psicosis cicloides: etiopatogenia, características clínicas y nosología. Aula Médica Psiquiatría, 10(1), 5-3. 
Ponce González, J. M., Velázquez Salas, A., Márquez Crespo, E., lópez Rodríguez, L., \& Bellido Moreno, M. ${ }^{a}$ L. (2009). Influencia del apoyo social en el control de las personas con diabetes. Index de Enfermería, 18(4), 224-228. https://doi.org/10.4321/S1132-12962009000400002

SERAFIn, M.R. y EsPiritu, M.I, (2013). Creación de validación de una Escala de complejidad de intervención desde el trabajo social sanitario, con adultos en un contexto hospitalario (ECISASH). Revista Aghatos. Atención Socio sanitaria y Bienestar, 1, 42-55.

Seva-Díaz, A., SeVA-Fernández, A. (2003). Los GRDs Psiquiátricos: Una investigación pendiente. European Psychiatry Journal, 17 (1), 49-63. https://doi. org/10.4321/S1579-699X2003000100006

VALLEJO, J. (2002). Introducción a la psicopatología y la psiquiatría. Barcelona: Elsevier Masson. 


\section{ENFOQUE Y ALCANCE. DIRECTRICES PARA AUTORES/AS}

\section{Enfoque y alcance}

Alternativas. Cuadernos de Trabajo Social tiene como objeto fundamental contribuir a la difusión de investigaciones, experiencias, trabajos teóricos y metodológicos, tanto de carácter académico como profesional, que se realizan en nuestro país y en el ámbito internacional, relativos al Trabajo Social, a la política social y a los Servicios Sociales, así como a otras disciplinas y profesiones que, desde un punto de vista multi e interdisciplinar, enriquecen y complementan la disciplina y la acción profesional del Trabajo Social en el ámbito de las Ciencias Sociales.

Los trabajos deben ser originales, no publicados ni estar siendo considerados en otra revista para su publicación, escritos en español, inglés, francés o portugués. El autor/a es el único responsable de las afirmaciones sostenidas en su artículo. De manera excepcional, los artículos que no sean inéditos se publicarán bajo la valoración del Comité Editorial y en función de: que su difusión haya sido en algún medio de difícil acceso y de que se consideren de particular relevancia e interés científico profesional.

La revista está integrada en RUA, Repositorio Institucional de la Universidad de Alicante y cumple con el estándar de metadatos Dublin Core (http://www.dublincore.org) y con el protocolo OAI-PMH (Open Archives Initiative - Protocol for Metadata Harvesting: http://www.openarchives.org) para la transmisión de contenidos en Internet.

Cada uno de sus números se edita tanto en versión impresa como en versión electrónica: esta última disponible en esta plataforma y en el Repositorio Institucional de la Universidad de Alicante.

En la sección Próximos artículos de esta edición electrónica se puede acceder a los manuscritos evaluados y aceptados para publicación en formato 'postprint'. Alternativas. Cuadernos de Trabajo Social está abierta a intercambios con otras publicaciones.

\section{Directrices para autores/as}

\section{Propuestas de artículos}

Se aceptan propuestas de artículos para publicar en la revista, ajustadas a su objeto y a sus normas de publicación: 
- Alternativas. Cuadernos de Trabajo Social tiene como objeto fundamental contribuir a la difusión de investigaciones, experiencias, trabajos teóricos y metodológicos, tanto de carácter académico como profesional, que se realizan en nuestro país y en el ámbito internacional, relativos al Trabajo Social, a la política social y a los Servicios Sociales, así como a otras disciplinas y profesiones que, desde un punto de vista multi e interdisciplinar, enriquecen y complementan la disciplina y la acción profesional del Trabajo Social en el ámbito de las Ciencias Sociales.

- La revista adopta y se adhiere a las normas de publicación establecidas en el Manual de la APA, adaptadas a las necesidades editoriales de la revista. Es preciso recordar que el cumplimiento de los requisitos del Manual de la APA facilita la indización de la revista en las principales bases de datos de la especialidad, con lo que ello supone de beneficio para los autores y sus centros por la mayor difusión que alcanzan los trabajos publicados.

Serán considerados para su publicación los siguientes tipos de trabajos: investigaciones originales, trabajos teóricos, experiencias prácticas y notas técnicas.

- Investigaciones originales: Estarán estructuradas de la siguiente manera: resumen, palabras clave, texto (introducción, metodología, resultados, discusión y conclusiones), agradecimientos y bibliografía. La extensión máxima del texto será de 8.000 palabras (en formato. doc), escritas a doble espacio, cuerpo de letra 12, tipo Times New Roman, admitiéndose 4 figuras y 6 tablas. Las tablas y figuras deberán presentarse en hoja aparte, numerándose correlativamente e indicando el lugar de colocación en el artículo. Si se utilizan, han de ser aquellas que, por su relevancia, sean necesarias para apoyar los argumentos recogidos en el texto. Es aconsejable que el número de autores/as no sobrepase los seis.

- Trabajos teóricos y experiencias prácticas: Los trabajos teóricos consistirán en un análisis crítico de temáticas relevantes para el Trabajo Social, la política social y los servicios sociales. Las experiencias prácticas consistirán en una sistematización rigurosa del proceso y resultados de las mismas. Ambas, revisiones y experiencias, deberán incluir un apartado con aportaciones o propuesta de aplicación o transferencia al Trabajo Social, la política social, los servicios sociales o la intervención social. Los trabajos dirigidos a esta sección se estructurarán del mismo modo que las investigaciones originales (introducción, metodología, 
resultados, discusión y conclusiones), con las adaptaciones necesarias para facilitar su comprensión. Los textos tendrán una extensión máxima de 4.000 palabras en formato.doc, escritas a doble espacio, cuerpo de letra 12 y tipo Times New Roman. Opcionalmente el trabajo podrá incluir tablas y figuras.

- Notas técnicas: Describirán de forma resumida normativas o políticas, investigaciones en curso, así como crónicas de congresos, seminarios o jornadas relevantes para el Trabajo Social, la política social y los servicios sociales. Tendrán una extensión máxima de 1.000 palabras (en formato.doc), escritas a doble espacio, cuerpo de letra 12 y tipo Times New Roman.

\section{Envío de los trabajos}

La recepción de artículos está abierta durante todo el año, a excepción del mes de agosto. Tanto el envío como la publicación de artículos son gratuitos.

\section{Idioma y formato de los trabajos}

Los trabajos podrán estar escritos en español, inglés, francés o portugués.

Los manuscritos deberán ajustarse al formato indicado antes para cada tipo de trabajo (investigaciones originales, trabajos teóricos, experiencias prácticas y notas técnicas).

Todo original se somete al arbitraje por pares académicos. Con la finalidad de garantizar la imparcialidad en la selección de los artículos para publicar, se observa el anonimato tanto de autores como de evaluadores. Para ello, el texto de los artículos deberá enviarse anonimizado: no debe incluirse el nombre de los/as autores/as ni su filiación, y se suprimirán (bajo el rótulo de *anonimizado*) todos los agradecimientos, referencias a proyectos financiadores o cualquier otro elemento que pudieran permitir directa o indirectamente la identificación del autor/a. La redacción de Alternativas. Cuadernos de Trabajo Social se asegurará de que los textos cumplen con esta condición. Si el artículo es aceptado para su publicación, la revista solicitará la versión no anonimizada junto a las eventuales modificaciones requeridas tras el proceso de revisión.

\section{Estructura de los trabajos}

Los manuscritos se presentarán según el siguiente orden y estructura: 
4.1. Página de título. Primera página del manuscrito

A modo de portada del manuscrito, esta primera página contendrá:

a) Título del artículo (conciso pero informativo), en español e inglés, conformado con el mayor número de términos significativos posibles (a ser posible tomados de un vocabulario controlado o tesauro de la especialidad). Si es necesario, se añadirá un subtítulo no superior a 40 letras y espacios, en español e inglés.

b) Nombre y dos apellidos de cada uno de los autores o autoras teniendo en cuenta la forma de firma para indexación en bases de datos internacionales (véanse en la sección «¿Sabes qué tienes que hacer para identificar tus publicaciones científicas?» las recomendaciones formuladas en http://www.accesowok.fecyt.es/).

c) Nombre completo del centro de trabajo de cada uno de los/as autores/ as, el cual tendrá su referencia al lado del nombre del/de la autor/a con números arábigos entre paréntesis (o en superíndice). Código ORCID de cada uno de los/as autores/as.

d) Nombre y dirección completa de la persona responsable del trabajo o del/de la primer/a autor/a como responsable de correspondencia, incluyendo número de teléfono, así como dirección de correo electrónico si se dispone de ella.

e) Información sobre becas, ayudas o soporte financiero con el que se ha contado (Proyectos de Investigación) para la subvención del trabajo y otras especificaciones, cuando sea el caso.

\subsection{Páginas de resumen y palabras-clave. Segunda página del manuscrito}

Una segunda página independiente deberá contener los nombres y apellidos de los/as autores/as, el título del artículo y el de la revista, un resumen del contenido del artículo en español y el listado de palabras clave. Tanto el resumen como las palabras clave tendrán una versión en inglés revisada por persona experta.

Para aquellos textos cuyo idioma sea diferente del inglés se debe enviar, además del resumen reducido de 150 palabras, un resumen extenso en inglés del orden de 1000 a 1500 palabras (revisado por persona experta) en el que se describa con algo más de profundidad los métodos, datos, hallazgos e implicaciones del trabajo.

a) En el caso de los artículos originales, el contenido del resumen constará de: introducción, metodología, resultados, discusión y conclusiones. 
En cada uno de ellos se describirán, de forma concisa, respectivamente, el motivo y el objetivo de la investigación, la metodología empleada, los resultados más destacados y las principales conclusiones y/o recomendaciones que se pueden extraer del trabajo. Se enfatizarán los aspectos novedosos y relevantes del trabajo.

b) Palabras clave: a continuación del resumen se especificarán cinco palabras clave o frases cortas que identifiquen el contenido del trabajo para su inclusión en los repertorios y bases de datos nacionales e internacionales. Se procurará poner el mayor número posible hasta un máximo de cinco. Deberán utilizarse términos controlados de referencia (a ser posible tomados de un vocabulario controlado o tesauro de la especialidad).

\subsection{Texto del manuscrito. Tercera página, que será la del arranque del texto del manuscrito}

La tercera página y siguientes serán las que se dediquen al texto del manuscrito, que se ajustará a las especificaciones de las instrucciones indicadas para cada tipo de trabajo. Los trabajos deben ajustarse en la medida de lo posible a los apartados ya indicados: Introducción, metodología, resultados, discusión, conclusiones y bibliografía:

- Introducción: Debe incluir los fundamentos y el propósito del estudio, utilizando las citas bibliográficas estrictamente necesarias. No incluirá datos o conclusiones del trabajo presentado. No debe realizarse una revisión bibliográfica detallada.

- Metodología: Será presentado con la precisión que sea conveniente para que el lector comprenda y confirme el desarrollo de la investigación. Fuentes y métodos previamente publicados deben describirse sólo brevemente y aportar las correspondientes citas, excepto si se han realizado modificaciones en los mismos. Cuando así proceda, se describirá el cálculo del tamaño de la muestra y la forma de muestreo utilizada en cada caso. Se hará referencia al tipo de análisis documental, crítico, estadístico, etc. empleado. Si se trata de una metodología original, será necesario exponer las razones que han conducido a su empleo y describir sus posibles limitaciones.

Cuando se trate de trabajos experimentales en los que se hayan utilizado grupos humanos, se indicará si se han tenido en cuenta los criterios éticos 
correspondientes. No deben utilizarse los nombres ni las iniciales de las personas que hayan participado formando parte de la muestra estudiada.

- Resultados: Aparecerán en una secuencia lógica en el texto, tablas o figuras, no debiendo repetirse en todas ellas los mismos datos. Se procurará resaltar las observaciones importantes. Se describirán, sin interpretar ni hacer juicios de valor, las observaciones efectuadas con el material y métodos empleados.

- Discusión: Resumirán los hallazgos, relacionando las propias observaciones con otros estudios de interés y señalando las aportaciones y limitaciones de unos y otros. No deben repetirse con detalle los datos u otro material ya comentado en otros apartados. Se mencionarán las inferencias de los hallazgos y sus limitaciones, incluyendo las deducciones para una investigación futura.

- Conclusiones: Resumirán las ideas-fuerza que se pueden extraer de los resultados y su discusión y sus posibles transferencias a la sociedad o a la práctica, así como las posibles recomendaciones, si las hubiere. Se enlazarán las conclusiones con los objetivos del estudio, evitando las afirmaciones gratuitas y las conclusiones no apoyadas completamente por los datos del trabajo.

- Agradecimientos: Únicamente se agradecerá su colaboración a aquellas personas que hayan hecho contribuciones sustanciales al estudio pero sin llegar a merecer la calificación de autor, debiendo disponer el autor de su consentimiento por escrito. Asimismo, se recomienda a los autores, en su caso, una declaración explícita de la fuente de financiación de la investigación, y que ésta se encuentre en los agradecimientos.

- Bibliografía: La bibliografía debe aparecer a continuación de la discusión y conclusiones, o de los agradecimientos si los hubiere, según el orden alfabético de autores y siguiendo el estilo basado en las normas de la APA. En el caso de publicaciones periódicas, incluir DOI.

A fin de asegurar la coherencia, en cualquier momento del proceso editorial, la dirección de la revista podrá requerir a los autores el envío de la primera página (fotocopia) de cada uno de los artículos citados en la bibliografía.

La bibliografía debe ser corregida por el autor, comparándola con la copia en su poder. Se evitará utilizar frases imprecisas como citas bibliográficas. No pueden emplearse como tales las que precisen de aclaraciones del tipo «observaciones no publicadas», ni «comunicación personal», aunque sí podrán citarse dentro del texto entre paréntesis. Los trabajos aceptados, pero aún 
no publicados, se incluirán en las referencias bibliográficas especificando el nombre de la revista, seguido por la expresión «en prensa».

Las citas bibliográficas en el cuerpo del manuscrito, cuyo número ha de ser el suficiente y necesario, se presentarán identificando autor y año entre paréntesis y separados por una coma (ejemplo: Coob, 1989).

Las citas textuales deben ir entrecomilladas cuando sean inferiores a tres líneas. Si tienen más de 3 líneas irán sin entrecomillar y sangradas por el margen izquierdo, indicando en todo caso entre paréntesis el apellido del autor o autores del texto, el año de publicación y la página (ejemplo: Coob, 1989: 25-27).

Para las revistas, dada su trascendencia para los índices de citas y los cálculos de los factores de impacto, se citarán: a) autor(es), con su(s) apellido(s) e inicial(es) de nombre(s), separando los apellidos del nombre con una coma. Si hay más de un autor, entre ellos se pondrá una coma. Todos hasta un máximo de seis, y cuando sean más de seis se pondrán sólo los seis primeros y se añadirá et al. Tras el último autor se pondrá el año entre paréntesis y un punto; b) título del artículo en su lengua original, y con su grafía y acentos propios. Tras el título se pondrá un punto; c) nombre correcto de la revista; d) número de volumen $\left(n .^{\circ}\right)$ (la separación entre este apartado y el siguiente se hará con coma); y e) páginas primera y última, separadas por un guión.

\subsection{Carta de presentación, de cesión de derechos y de declaración de conflicto de intereses}

\section{Sr./Sra. director/a de la Revista Alternativas. Cuadernos de Trabajo Social}

Leídas atentamente las instrucciones a autores, y analizada la cobertura de la revista, considero que la publicación que Vd. dirige es la adecuada para la difusión de nuestro trabajo, por lo que le ruego someta a la consideración de su posible publicación en la sección de el manuscrito que adjunto le remito titulado , cuyos autores son de los cuales será el encargado de correspondencia.

Las aportaciones originales y novedades que en nuestra opinión el referido manuscrito aporta al estudio de son, en síntesis, las siguientes: 
Los autores certifican que este trabajo no ha sido publicado ni en todo ni en parte por cualquier otro medio, ni está en vías de consideración para publicación en otra revista.

Los autores se responsabilizan de su contenido y de haber contribuido a la concepción, diseño y realización del trabajo, análisis e interpretación de datos, y de haber participando en la redacción del texto y sus revisiones así como en la aprobación de la versión que finalmente se remite.

Asimismo, aceptamos la introducción de cambios en el contenido si hubiere lugar tras la revisión y de cambios en el estilo del manuscrito por parte de la redacción de la revista.

\section{Cesión de derechos y declaración de conflicto de intereses}

Los autores abajo firmantes transfieren los derechos de propiedad (copyright) del presente trabajo al Servicio de Publicaciones de la Universidad de Alicante, como organización editora patrocinadora de la revista Alternativas. Cuadernos de Trabajo Social.

Declaramos además estar libres de cualquier asociación personal o comercial que pueda suponer un conflicto de intereses en conexión con el artículo remitido, así como el haber respetado los principios éticos de investigación.

Firmado:

\section{Autor/a XXXX-XXXXX; Autor/a XXXXXXX; Autor/a XXXXX-XXXXXXX}

\section{Lista de comprobación para la preparación de envíos}

Como parte del proceso de envío, los autores/as están obligados a comprobar que su envío cumpla todos los elementos que se muestran a continuación. Se devolverán a los autores/as aquellos envíos que no cumplan estas directrices.

1) El texto ha sido anonimizado: no incluye el nombre de los/as autores/ as ni su filiación, y se han suprimido (bajo el rótulo de *anonimizado*) todos los agradecimientos, referencias a proyectos financiadores o cualquier otro elemento que pudieran permitir directa o indirectamente la identificación del autor/a.

2) Se envía el manuscrito en formato.doc, incluidas las citas bibliográficas, tablas y figuras. 
3) Se han seguido las instrucciones para Asegurar una evaluación anónima.

4) Se adjunta como archivo complementario la carta de presentación, indicando originalidad, novedad del trabajo, sección de la revista a la que se dirige, responsabilidad de la autoría, no haber sido publicado ni en todo ni en parte por cualquier otro medio, ni estar en vías de consideración en otra revista, aceptación de la introducción de cambios en el contenido si hubiere lugar tras la revisión y de cambios en el estilo del manuscrito por parte de la redacción de la revista, cesión de los derechos de autor al editor, no existencia de conflicto de intereses así como haber respetado los principios éticos de investigación.

5) Siempre que ha sido posible, se han proporcionado direcciones URL para las referencias.

6) El texto tiene interlineado sencillo; 12 puntos de tamaño de fuente; se utiliza cursiva en lugar de subrayado (excepto en las direcciones URL); y todas las ilustraciones, figuras y tablas se encuentran colocadas en los lugares del texto apropiados, en vez de al final. En su caso, las figuras y tablas aportan información adicional y no repetida en el texto.

7) El texto se adhiere a los requisitos estilísticos y bibliográficos resumidos en las Directrices del autor/a, que aparecen en la sección Acerca de la revista.

8) Se incluye el título completo, un resumen reducido y las palabras clave (entre 1 y 5 palabras clave), todo ello en español e inglés y, en su caso, francés o portugués. Cuando el texto completo no esté escrito en lengua inglesa, se incluirá un resumen extenso en inglés. Los textos en lenguas extranjeras deberán estar revisados por personas expertas. Ambos resúmenes se ajustan a las normas.

9) Se incluyen las filiaciones institucionales y/o profesionales de cada uno de los autores así como la dirección del autor principal o responsable de correspondencia, número de teléfono, fax y dirección de correo electrónico.

10) En su caso, se declaran becas, ayudas o soporte financiero y su procedencia.

11) El manuscrito responde a la estructura exigida en las normas de publicación y a las especificaciones de la sección a la que se dirige.

12) El manuscrito describe todas las fuentes, materiales, equipo y elementos usados, tanto en términos de grupos investigados como la globalidad del estudio. En su caso, una persona experta en el contenido del manuscrito podría reproducir su estudio, experimento, análisis, etc., a partir de la metodología descrita. En su caso, se han utilizado 
análisis estadísticos, y estos han sido revisados por persona experta en la materia. Las conclusiones se apoyan en los resultados obtenidos.

13) Se han revisado las referencias bibliográficas, y se ajustan en su estilo y formato a las normas internacionales APA exigidas por la revista.

14) Se citan las normas éticas utilizadas.

\section{Aviso de derechos de autor/a}

Los autores transfieren los derechos de propiedad (copyright) del presente trabajo al Servicio de Publicaciones de la Universidad de Alicante, como organización editora patrocinadora de la revista Alternativas. Cuadernos de Trabajo Social.

Los trabajos se publican bajo una licencia de Creative Commons Reconocimiento-NoComercial 4.0 Internacional, salvo que se indique lo contrario. Esto es que se puede compartir y adaptar el material siempre que medie atribución del autor/es, del primer medio que publica y se proporcione un enlace a la licencia. Igualmente hay que indicar si se han realizado cambios. No se permite el uso del material con fines comerciales.

A efectos de lo estipulado en los artículos 138-143 de la Ley de Propiedad Intelectual, la publicación de un trabajo que atente contra dichos derechos será responsabilidad del autor/a. El Comité Editorial de Alternativas. Cuadernos de Trabajo Social no se hace responsable, en ningún caso, de la credibilidad y autenticidad de los trabajos. Del mismo modo, las opiniones y hechos expresados en cada artículo son de exclusiva responsabilidad de sus autores/as y Alternativas. Cuadernos de Trabajo Social no se identifica necesariamente con ellos.

\section{Declaración de privacidad}

Los nombres y las direcciones de correo electrónico introducidos en esta revista se usarán exclusivamente para los fines establecidos en ella y no se proporcionarán a terceros o para su uso con otros fines. 


\section{FOCUS AND SCOPE. AUTHOR GUIDELINES}

\section{Focus and Scope}

The main purpose of Alternativas. Cuadernos de Trabajo Social is to contribute to the dissemination of research findings, experiences, theoretical and methodological work of an academic and professional nature which are carried out in Spain and at an international level. They are also related to social work, social policy and social services, as well as to other disciplines and professions that from a multi- and interdisciplinary point of view to enrich and complement the discipline and the professional activity of social work in the field of social sciences.

The work must be original, unpublished and not be under consideration by any other journal in order to be published. They must be written in Spanish, English, French or Portuguese. The author is solely responsible for the statements in the article. Exceptionally, previously published articles will be only be republished under assessment of the Editorial Board and depending on whether its dissemination occurred through an inaccessible media or depending on its relevance and professional scientific interest.

The journal is available in RUA, Repositorio Institucional de la Universidad de Alicante (Institutional Repository of the University of Alicante) and complies with the Dublin Core metadata standard (http://www.dublincore.org) and with the OAI-PMH protocol (Open Archives Initiative - Protocol for Metadata Harvesting: http://www.openarchives.org) for the transmission of content on the Internet.

Each of its issues is edited in paper format as well as in digital format: the latter is available on this platform and in the Institutional Repository of the University of Alicante.

In the section Next articles of this electronic edition, you can find all the evaluated and accepted manuscripts in order to be published in 'postprint' format. Alternativas. Cuadernos de Trabajo Social is open to exchanges with other publications.

\section{Author Guidelines}

\section{Article proposal}

Articles to be published to be published, if they are in accordance with the purpose and publication rules of the journal: 
- The main purpose of Alternativas. Cuadernos de Trabajo Social is to contribute to the dissemination of research findings, experiences, theoretical and methodological work of an academic and professional nature which are carried out in Spain and at an international level. They are also related to social work, social policy and social services, as well as to other disciplines and professions that from a multi- and interdisciplinary point of view enrich and complement the discipline and the professional activity of social work in the field of social sciences.

- The journal adopts and adheres to the publication rules stablished in the APA manual, adapted to the editorial needs of the journal. It should be recalled that the fulfilment of the APA manual requirements enhances the indexation of the journal in the principal data bases of the specialty, which is beneficial for the authors and its centres because of the broadest dissemination that the published works can reach.

The following kinds of works will be considered to be published: original research, theorical works, practical experiences and technical notes.

- Original research: They will be structured in the following manner: abstract, key words, text (introduction, methodology, results, discussion, conclusions) acknowledgements and bibliography. The maximum length of the text must be 8.000 words (in.doc format), typed with double-spacing, font size 12, type Times New Roman, being admitted 4 figures and 6 tables. The tables and figures must be presented on a separated sheet, be numbered correlatively and indicate where they are in the article. If they are used, they must be those that, according to its relevance, are needed to support the arguments shown in the text. It is advisable that the number of authors is not higher than six.

- Theorical works and practical experiences: the theoretical works will consist in a critical analysis of relevant topics for social work, social policy and social services. The practical experiences will consist in a rigorous systematization of the process and of the results. Both, reviews and experiences, will include a section with contributions or implementation proposal or its possible uses in social work, social policy, social services or social intervention. The works directed to this section will be structured in the same way as the original investigations (introduction, methodology, results, discussion and conclusions), with the necessary adaptations to facilitate their understanding. The maximum length of the text will be 4.000 words in.doc format), typed with 
double-spacing, font size 12 and type Times New Roman. Optionally the work will include tables and figures.

- Technical notes: they will describe in a summarised form regulations and policies, in-progress investigations, as well as reports on congress, seminars and conferences that are relevant for social work, social policies and social services. The maximum length will be 1.000 words (in. doc format), typed with double-spacing, font size 12 and type Times New Roman.

\section{Submission of works}

Articles will be received along the whole year, except in August. Both the submission and the publication of the articles are free of charges.

\section{Language and format}

The works can be written in Spanish, English, French or Portuguese.

The manuscripts must be adjusted to the format previously indicated for each type of work (original investigation, theorical works, practical experiences and technical notes.

All the original articles are subject to arbitration by academic peers. In order to ensure the impartiality of the selection of articles to be published, the authors and the evaluators always remain anonymous. For this purpose, the text of the articles must be sent anonymized: it must not include the name of the author(s) or their filiation, and all acknowledgements will be removed (by using the word anonymized), as well as all references to funded projects or any other element that could reveal directly or indirectly the author's identity. The editorial team of Alternativas. Cuadernos de Trabajo Social will ensure that all the texts meet this condition. If the article is accepted to be published, the journal will ask for a non-anonymized version of the article along with the possible required modifications after the review process.

\section{Structure of the works}

The manuscripts will be presented with the following order and structure:

\subsection{Title page. First page of the manuscript.}

As title page of the manuscript, this first page will have:

a) Name of the article (concise and informative), in Spanish and English, written with as much significant terms as possible (if possible using a 
controlled vocabulary and thesaurus of the speciality). When needed, it will be added a subtitle that is not longer than 40 words (spaces included) in Spanish and English.

b) Name and surname of each of the authors taking into account their signature for indexation in international databases (refer to the section " ¿Sabes qué tienes que hacer para identificar tus publicaciones científicas?» and see the suggestion made in http://www.accesowok.fecyt.es/).

c) Whole name of the workplace of each author(s), which will have its reference next to the name of the author(s) with Arabic numeral in brackets (or superscripted). Open Researcher and Contributor ID (ORCID) of each author(s).

d) Name and whole address of the responsible person for the work or the first author as the responsible of correspondence, including telephone number, as well as e-mail address when available.

e) Information about scholarships, grants or financial aids received (Research Projects) for the subvention of the work and other specifications, when needed.

\subsection{Abstract pages and key-words. Second page of the manuscript.}

An independent second page must have the whole name of the author(s) written, the title of the article and of the journal, an abstract of the content of the article in Spanish and a list of key-words. Both the abstract and the words must have a translation into English reviewed by an expert in the field.

For those texts which language is other different than English will need, apart from the brief abstract of 150 words, an extended abstract in English of 1000-1500 words (reviewed by an expert in the field) in which the methods, data, results and implication of the work are described in detail.

a) In the case of original articles, the content will consist of: introduction, methodology, results, discussion and conclusions. In each of them will be described in a concise manner the reason and objective of the investigation, the methodology used, the most significant results and the main conclusions and/or recommendations that can be drawn from the work, respectively. The new and relevant aspects of the work will be emphasized.

b) Key words: after the abstract, five key words or short sentences will be specified, identifying the content of the work in order to include it in indexes and national and international databases. The article should include the highest possible number of words, up to a maximum of 
five. Controlled terms of reference must be used (if possible from a controlled vocabulary or a thesaurus of the speciality).

4.3. Text of the manuscript. The third page will be the beginning of the text of the manuscript.

The third page and the following will contain the text of the manuscript, conforming to the specifications of the instructions given for each type of work. Works must have the following sections (as previously indicated): introduction, methodology, discussion, conclusions and bibliography.

- Introduction: it must include the fundamentals and purpose of the study, using the strictly needed bibliographic citations. It won't include data or conclusions of the presented work. A detailed bibliographical review is not needed.

- Methodology: it will be presented with the convenient precision for the reader to understand and confirm the development of the investigation. Sources and methods previously published must be described briefly and provide the corresponding citation, except when changes were made. Where appropriate, the calculation of the sample size will be described along with the sampling mode used in each case. The type of analysis used (documental, critical, statistic, etc.) will be mentioned. In case of an original methodology, it will be necessary to explain the reasons that lead to its use and describe its possible limitations.

When human samples are used, it will be indicated whether the correspondent ethical criteria were taken into account. No names nor initials of the people from the sample should be used.

- Results: they will appear in a logic order in the text, tables or figures, not repeating the same data in them. Important observations should be highlighted. The observation made with the material and methodology will be described, without interpretation nor value judgements.

- Discussion: findings will be summarised, linking own observation with other studies of interest, pointing out the contributions and limitations of both. Detail of the data must not be repeated nor from other material previously mentioned. The implications of the findings and its limitations should be included, as well as the deductions for future investigations. 
- Conclusions: the main ideas obtained from the results, its discussion and its possible applications will be summarised, as well as the possible suggestions, where they exist.

- Acknowledgments: only those who made substantial contributions to the study without being considered author will be acknowledge, when the author gives the correspondent written consent. Furthermore, it is recommended, where necessary, that authors explicitly declare their source of financing for the investigation, and also that it is found in the acknowledgements.

- Bibliography: The bibliography must appear after the discussion or conclusions (or after the acknowledgments if any, in alphabetical order of the authors and following the APA rules. In case of periodical publications, include DOI.

To assure coherence, in any moment of the editing process, the direction of the journal can request that the authors send the first page (photocopy) of all of the articles mentioned in the bibliography.

The bibliography must be corrected by the author, comparing it with the one in his possession. Imprecise sentences, such as bibliographical references, should not be used. Bibliography cannot be those which need clarification such as «non-published observations», nor "personal communication», though they may be used along the text in parenthesis. The accepted works, which are not published yet, will be included in the bibliography specifying the name of the journal, followed by the expression «in press».

Citations in the body of the manuscript (there must be enough) will be presented identifying the author and the year in parenthesis and separated with a comma (example: Coob, 1989).

Quotes must be enclosed within double quotation marks when they are no longer than three lines. If they are longer that three lines they will not be enclosed with quotation mark and left indented, always indicating in parenthesis the surname of the author or authors of the text, the publication year and the page (example: Coob, 1989: 25-27).

For journals, in accordance with its relevance to citations index and the calculation of the impact factors, it should be quoted: a) author(s), with their surname(s) and name's initial, separating the surnames with a comma. If there is more than one author, the names will be separated with a comma. When there are more than six authors, only the first six will be written followed by et al. After the last author the year will be written in parenthesis and a full stop; b) title of the article in its original language, and with its own spelling 
and grammar. After the title a full stop will be written; c) correct name of the journal; d) number of the volume $\left(n^{\circ}{ }^{\circ}\right.$ ) (the division between this section and the following will be made with a comma); and e) first and last page, separated with a hyphen.

For the examples of bibliographical references quoted according to the necessities of the journals, see this link.

\subsection{Letter of presentation, copyright form and disclosure of conflict of interest.}

To the Editor in Chief of the Journal Alternativas. Cuadernos de Trabajo Social

Having carefully read the Authors' Instructions and analyzed the coverage of the Journal, I believe that the publication that you manage is appropriate for disseminating our work. I therefore request that you take into consideration for possible publication in the section the attached manuscript titled

The authors are , with being responsible for correspondence.

The original and new contributions made, in our opinion, be that above-mentioned manuscript to the study of are summarized below:

The authors certify that this work has not been published, in whole or in part, in any other medium, nor is it under consideration for publication in any other journal.

The authors are liable for its content and for having contributed to the conception, design and execution of the work, analysis and data interpretation, and for having participated in writing and reviewing the text, as well as approving the final version to be submitted.

Likewise, we accept the introduction of changes to the content, if necessary subsequent to review, and of changes to the style of the manuscript by the journal's editorial staff. 


\section{COPYRIGHT TRANSFER AND CONFLICT OF INTEREST STATEMENT}

The undersigned authors transfer the copyright for this work to Publications Service of Alicante University as the publishing organization that sponsors the Journal Alternativas. Cuadernos de Trabajo Social.

We further state that we are free of any personal or business association that could represent a conflict of interest regarding the article submitted, and we have respected the research ethics principles.

Signed:

Author XXXX-XXXXX; Author XXXXXXX; Author XXXXX-XXXXXXX

\section{Submission Preparation Checklist}

As part of the submission process, authors are required to check off their submission's compliance with all of the following items, and submissions may be returned to authors that do not adhere to these guidelines.

1) The text has been anonymized: the name(s) of the author(s) or their filiation is not included and all acknowledgements have been removed (by using the word *anonymized*), as well as all references to funded projects or any other element that could reveal directly or indirectly the author's identity.

2) The manuscript must be sent in.doc format, including bibliographical references, tables and figures.

3) The instructions to Ensure an anonymous evaluation must be followed.

4) A letter of presentation must be attached with the following: an explanation of the originality and novelty of the work, the section of the journal to which it's addressed, and authorship responsibility. It must certify that the work has not been previously published neither completely nor partly in any other means, or be in process of consideration by any other journal. It must state that the authors accept changes in the content (if any) after the revision and changes in style by the editors of the journal, as well as, transfer of copyright from the author to the editor, no conflict of interests and respected ethical research principles have been respected.

5) When possible, URL addresses are given for the references.

6) The text must have single line spacing; size 12 font; italics are used instead of underlining (except URL addresses); and all the images, figures and tables must be properly placed along the text, instead of at 
the end. The figures and tables must provide additional information not repeated in the text.

7) The text follows the style and bibliographical requirements summarized in the Guidelines for Authors, that can be found in the section About the journal.

8) The whole title, a reduced abstract, the key words (1-5 key words) in Spanish and English or in French or Portuguese must be included. An extended abstract in English must be included. All texts written in foreign languages must be revised by an expert. Both abstracts must comply with the publication norms.

9) The institutional and/or professional affiliations of each of the authors must be included, as well as, the address, telephone number, fax and email address of the main author or the person in charge of the correspondence.

10) Information about scholarships, grants or financial support must be stated.

11) The manuscript complies with the required structure in the publication standards and with the specifications of the section to which it's addressed.

12) The manuscript describes all the sources, materials, equipment and elements used, both in terms of researched groups and the complete study. An expert in the content of the manuscript could repeat the study, experiment, analysis, etc. based on the methodology described. If necessary, statistical analysis has been used and has been reviewed by an expert in the field. The conclusions are based on the results obtained.

13) The bibliographical references have been reviewed and comply in style and format with the international APA standards required by the journal. Abbreviations must be explained when first used in the text.

14) Ethical standards used must be quoted.

\section{Copyright Notice}

The authors transfer the copyright of the present work to the Publishing Service of the University of Alicante (Servicio de Publicaciones de la Universidad de Alicante), as publishing organization which sponsors the journal Alternativas. Cuadernos de Trabajo Social.

Works are published with a Creative Commons Attribution-NonCommercial 4.0 International Public License, unless stated otherwise. This means that the 
material can be shared and adapted provided that acknowledgement is given to the authors, to the first medium that has published the work, and that a link is provided to the licence. It should also be made clear whether any changes have been made. Use of the material for commercial purposes is not permitted.

For the purposes laid down in the articles 138-143 of Intellectual Property Law, the publication of a work which violates these rights will be responsibility of the author. The Editorial Board of Alternativas. Cuadernos de Trabajo Social is not responsible in any case for the credibility and authenticity of the works. In the same way, the opinions and facts mentioned in the articles are the sole responsibility of their authors and Alternativas. Cuadernos de Trabajo Social do not necessarily identify with them.

\section{Privacy Statement}

The names and email addresses included in this journal are to be used solely for the purposes established herein and must not be provided to third parties or be used for other purposes. 


\section{PROCESO DE EVALUACIÓN POR PARES}

Se trata de una revista arbitrada que utiliza el sistema de revisión externa por expertos/as (peer-review) en el conocimiento de los objetos investigados y en las metodologías utilizadas en las investigaciones, con la finalidad de alcanzar los mayores estándares de calidad científica en los trabajos publicados.

El Comité Editorial de Alternativas. Cuadernos de Trabajo Social no se hace responsable, en ningún caso, de la credibilidad y autenticidad de los trabajos. Del mismo modo, las opiniones y hechos expresados en cada artículo son de exclusiva responsabilidad de sus autores/as y Alternativas. Cuadernos de Trabajo Social no se identifica necesariamente con ellos.

Las obras recibidas pasarán por el siguiente proceso editorial en el que cada paso requiere la superación del anterior:

- Acuse de recibo. La revista acusa recepción de todos los trámites realizados para tener informados a los autores/as de la situación en la que se encuentra su manuscrito y, posteriormente, informará de su aceptación o rechazo.

- Revisión del Comité Editorial. El Comité Editorial pasará a considerar el trabajo para su publicación, comprobando si se adecua a la cobertura de la revista y cumple las normas de publicación. En tal caso, se procederá a su revisión externa (peer-review).

- Revisión por pares. Los manuscritos serán revisados de forma anónima (doble ciego) por dos personas externas, expertas en el objeto de estudio y/o metodología empleada, con arreglo al formulario de revisión de originales. El Comité Editorial de la revista, a la vista de los informes externos, se reserva el derecho de aceptar / rechazar los artículos para su publicación, así como el de introducir modificaciones de estilo y/o acortar los textos que sobrepasen la extensión permitida, comprometiéndose a respetar el contenido del original.

En el caso de juicios dispares entre las dos personas evaluadoras, los trabajos se remitirán a un tercer evaluador/a. Los trabajos que sean revisados y pudieran ser considerados para publicación previa modificación, deberán ser devueltos en el plazo de 30 días tanto si se solicitan correcciones menores como mayores. Cuando sea necesario, la nueva versión del texto será enviada otra vez a las personas evaluadoras, procedimiento que se seguirá hasta la aceptación definitiva del trabajo por la revista. Los autores/as recibirán los informes de evaluación de los revisores/as (de acuerdo con el protocolo de evaluación de la revista) de forma anónima, para que puedan realizar las correcciones o réplicas oportunas. 
Decisión del Comité Editorial. En general, una vez vistos los informes externos, los factores en los que se funda la decisión sobre la aceptación-rechazo de los trabajos por parte de la Redacción de la revista son los siguientes:

a) originalidad: totalmente original, información valiosa, repetición de resultados conocidos;

b) actualidad y novedad;

c) relevancia: aplicabilidad de los resultados para la resolución de problemas concretos;

d) significación: avance del conocimiento científico;

e) fiabilidad y validez científica: calidad metodológica contrastada;

f) presentación: buena redacción, organización (coherencia lógica y presentación material).

Revisión de los artículos aceptados. Una vez maquetado el artículo se le enviará al autor o autora una copia en formato pdf para que compruebe si es correcto, o hace falta alguna modificación. Únicamente se pueden realizar correcciones relacionadas con la prueba de maquetación del manuscrito original. Si en 72 horas no hay respuesta, se entenderá que el artículo está listo para su publicación. No se pueden realizar cambios en el mismo después de su publicación online.

Duración del proceso editorial. El proceso de evaluación puede tener una duración de entre 4 y 6 meses.

Publicación de los artículos aceptados. Los artículos evaluados y aceptados serán publicados tanto en la versión impresa como en la versión electrónica de la revista: esta última disponible en esta plataforma y en el Repositorio Institucional de la Universidad de Alicante (RUA). Entre tanto se publica la versión definitiva de cada número, los artículos evaluados y aceptados serán publicados en formato "postprint» en la sección «Próximos artículos» de la edición electrónica de la revista, con su correspondiente DOI.

En su caso, el autor/a o primer firmante recibirá un ejemplar de la revista en papel después de su publicación.

Dudas o aclaraciones. Para cualquier duda o aclaración, pueden dirigirse a la página de contacto. 


\section{Frecuencia de publicación}

Fundada con el mismo título en 1992 (números 1 al 15) por la Escuela Universitaria de Trabajo Social de la Universidad de Alicante, se publica desde el año 2009 (desde el número 16) por el Departamento de Trabajo Social y Servicios Sociales de dicha universidad.

Su periodicidad es anual (Diciembre). La recepción de artículos está abierta durante todo el año, a excepción del mes de agosto. Tanto el envío como la publicación de artículos son gratuitos.

Entre tanto se publica la versión definitiva de cada número, los artículos evaluados y aceptados serán publicados en formato "postprint» en la sección «Próximos artículos» de la edición electrónica de la revista, con su correspondiente DOI.

\section{Política de acceso abierto}

Esta revista proporciona un acceso abierto inmediato a su contenido, basado en el principio de que ofrecer al público un acceso libre a las investigaciones ayuda a un mayor intercambio global de conocimiento.

\section{Archivar}

Esta revista utiliza el sistema LOCKSS para crear un sistema de archivo distribuido entre bibliotecas colaboradoras, a las que permite crear archivos permanentes de la revista con fines de conservación y restauración.

\section{Responsabilidades éticas}

La publicación de artículos en una revista con revisión por pares es un reflejo directo de la calidad del trabajo de sus autores/as, y del compromiso y cualificación de los investigadores e investigadoras que actúan como revisores/as. Por ello, es responsabilidad y deber del Comité Editorial de la revista Alternativas. Cuadernos de Trabajo Social recordar a los/as autores/as que es una publicación comprometida con los principios éticos de la actividad científica en los siguientes términos:

\section{Publicación y autoría}

Todos los artículos deben incluir un listado de referencias, así como indicar si han recibido apoyo económico. Los trabajos deben estar libres de plagio o 
fraude científico, cuyos supuestos* se enumeran de manera no exhaustiva a continuación:

- Plagio: copia literal sin entrecomillar y citar la fuente; copia sustancial (materiales de investigación, procesos, tablas...); parafrasear o reproducir ideas sin citar la fuente y/o cambiando el significado original; reutilizar y enviar textos propios ya publicados sin indicar la fuente y el parafraseo abusivo incluso citando la fuente.

- Fraude científico: no reconocimiento de todos los investigadores/ as participantes en la elaboración del trabajo, el envío simultáneo a varias publicaciones, la división de un trabajo en partes diferentes que comparten las mismas hipótesis, población y métodos, así como la utilización de datos falsos o no probados. Finalmente, los autores/as deben declarar a la revista los potenciales conflictos de interés cuando envían un trabajo.

* Fuente: http://www.ethics.elsevier.com/

\section{Responsabilidad de los autores/as}

El envío de trabajos a Alternativas. Cuadernos de Trabajo Social supone la lectura y aceptación de las normas editoriales y de publicación de la revista, incluida la participación en un proceso anónimo de evaluación por pares.

Todos/as los/as autores/as que firman un trabajo deben haber contribuido de manera significativa a su elaboración y deben estar de acuerdo con el resultado final y con el envío del trabajo para su evaluación. Haber ayudado en la recolección de datos o haber participado en alguna técnica no son, por sí mismos, criterios suficientes para figurar como autor/a. En general, para figurar como autor/a deben cumplirse los siguientes requisitos:

a) Haber participado en la concepción y realización del trabajo que ha dado como resultado el artículo en cuestión.

b) Haber participado en la redacción del texto y en las posibles revisiones del mismo.

c) Haber aprobado la versión que finalmente va a ser publicada.

La revista declina cualquier responsabilidad sobre posibles conflictos derivados de la autoría de los trabajos a publicar.

- Los trabajos deben reconocer a todos/as los/as autores/as que han participado en su elaboración. 
- Los datos utilizados en el artículo deben ser reales y auténticos.

- Los/as autores/as asumen la obligación de corregir y/o retractarse ante posibles errores detectados posteriormente.

- Los artículos han de ser inéditos y no pueden ser enviados simultáneamente a ninguna otra publicación.

- Los/as autores/as han de declarar cualquier asociación comercial que pueda suponer un conflicto de intereses en conexión con el artículo remitido.

- Los/as autores/as deben mencionar, en la sección de metodología, que los procedimientos utilizados con las personas participantes han sido realizados tras obtención de un consentimiento informado, cuando así proceda.

- Los/as autores/as deben contar con permiso de publicación por parte de la institución financiadora de la investigación, cuando proceda.

- Los/as autores/as son responsables de obtener los oportunos permisos para reproducir parcialmente material (texto, tablas o figuras) de otras publicaciones y de citar su procedencia correctamente.

- Cuando se describen experimentos realizados en seres humanos debe indicarse si los procedimientos seguidos son conformes a las normas éticas del comité de experimentación humana responsable (institucional o regional) y a la Declaración de Helsinki de 1975 revisada en el 2000. No se deben utilizar nombres, iniciales u otros datos de identificación de los centros donde se ha realizado.

\section{Proceso de revisión.}

Todos los artículos enviados a la revista se someten a un proceso de revisión por pares con las siguientes características:

- La selección de los revisores se realiza en función de normas y principios previos basados tanto en su cualificación como en la calidad de su producción científica.

- El proceso de revisión será totalmente anónimo tanto para los autores como para los revisores. Los artículos y sus revisiones serán tratados confidencialmente.

- Los revisores consideran, entre sus criterios de evaluación, el respeto a los principios éticos esenciales en la investigación científica.

- Los juicios expresados en las revisiones deben ser objetivos.

- Tanto autores como revisores deben revelar las relaciones y fuentes de financiación que puedan generar potenciales conflictos de intereses. 


\section{Responsabilidades de los editores}

- El Comité Editorial tiene la responsabilidad y autoridad para aceptar o rechazar un artículo basándose en las revisiones.

- El Comité Editorial revelará en su caso las relaciones o fuentes de financiación que puedan ser potencialmente consideradas como conflictos de intereses respecto a los artículos que rechaza o acepta.

- Sólo se aceptarán los artículos en los que existe una evidencia cierta sobre el cumplimiento de las normas editoriales.

- El Comité Editorial se reserva el derecho de retirar cualquier trabajo recibido, aceptado o ya publicado en caso de constatarse plagio, falsificación o publicación duplicada, así como los diversos supuestos de fraude científico anteriormente enumerados. Del mismo modo, promoverá la publicación de correcciones o retractaciones frente a errores detectados.

- El Comité Editorial se compromete a preservar el anonimato de los revisores de manera que nunca puedan asociarse con los artículos revisados.

\section{Cuestiones éticas de publicación}

El Comité Editorial se compromete a:

- Vigilar y preservar los principios éticos de publicación.

- Evitar la publicación de material plagiado o elaborado de manera fraudulenta.

- Estar abierto a la publicación de correcciones, clarificaciones, retractaciones y disculpas siempre que sea necesario.

- Ofrecer apoyo en el proceso de retractación de artículos.

- Realizar todas las acciones necesarias para cumplir los estándares de compromiso intelectual y ético.

\section{Exención de responsabilidad}

Tanto el Comité Editorial como la empresa editora declinan cualquier responsabilidad sobre el material publicado. Ni el Comité Editorial ni la empresa editora garantizan o apoyan ningún producto que se anuncie en la revista, ni garantizan las afirmaciones realizadas por el fabricante sobre dicho producto o servicio. 


\section{PEER REVIEW PROCESS}

Alternativas is an arbitrated journal which uses an external review process carried out by experts (peer-review). These experts are specialized in the knowledge of the researched subjects and in the methodologies used during research with a view to achieve the greatest quality standards in scientific quality in the published works.

The Editorial Board of Alternativas. Cuadernos de Trabajo Social is in no case responsible for the credibility and authenticity of the works. In the same way, the opinions and facts presented in each article are the sole responsibility of their authors.

The received works are subject to the following editing process, in which each step requires to overcome the previous one:

- Acknowledgement of receipt. The journal acknowledges the receipt of the completed procedures in order to keep the authors informed about the situation of their manuscripts and subsequently they will be informed about its approval or rejection.

- Review of the Editorial Board. The Editorial Board will consider the work for publication by verifying if the work is appropriate for the coverage of the journal and if it satisfies the publication standards. In that case, the external review (peer-review) will take place.

- Peer-review. The manuscripts will be reviewed anonymously (double blind) by two external reviewers, who are experts in the object of study and/or methodology used following the revision form of the original. In the view of external reports, the Editorial Board of the journal reserves the right to accept/refuse the articles for their publication, as well as the right to introduce style modifications and/or to shorten the texts, which exceed the text length permitted, by committing itself to respect the original content.

In the case of different opinions between the two evaluators, the works will be sent to a third evaluator. The works reviewed that could be considered to be published with prior modification must be returned within a period of 30 days, in the case of either minor or major corrections. When necessary, the new version of the text will be sent again to the evaluators. This process will be followed until there is definitive approval of the work by the journal. The author(s) will receive the evaluation reports of the evaluators anonymously (according to the protocol of the journal) so that they can make the appropriate corrections or copies. 
Decision of the Editorial Board. In general, once the external reports are seen, the decision of approval-rejection of works on the part of the Editorial Board is based on the following:

a) originality: totally original, valuable information, repetition of known results;

b) current and new;

c) relevance: applicability of the results for specific problem-solving;

d) significance: progress of scientific knowledge;

e) reliability and scientific validity;

f) presentation: good writing skills, organization (logical coherence and presentation).

Review of the accepted articles. When the article is ready for print, proofs will be sent to the author in a pdf file for revision. The only corrections allowed concern the formatting of the article. Authors have 72 hours to reply. If there is no reply, it is understood that the article is ready for publication. No changes are allowed to articles after publication online.

Duration of the editing process. The editing process can last between 4 and 6 months.

Publication of the accepted articles. The evaluated and accepted articles will be published in paper format as well as in digital format: the latter is available in this platform and in the Institutional Repository of the University of Alicante (RUA). As long as the definitive version of each issue is published, the evaluated and accepted articles will be published with its respective DOI in «postprint» format in the section «Next articles» in the electronic edition of the journal.

After the publication, the author or first signatory will receive a copy of the journal in paper format.

Doubts and questions. If you have any doubt or question, please contact us (see Contact section).

\section{Publication Frequency}

The journal was founded with the same name in 1992 (issues from 1 to 15) by the Graduate School of Social Work of the University of Alicante. The journal has been published since 2009 (from issue 16) by the Department of Social Work and Social services of the same university. 
It's published annually (December). The receipt of articles is open throughout the year, with the exception of August. The submission and publication of an article is free of charge.

As long as the definitive version of each issue is published, the evaluated and accepted articles will be published with its respective DOI in "postprint» format in the section «Next articles» in the electronic edition of the journal.

\section{Open Access Policy}

Immediate access to the journal's contents is granted upon the belief that free access to research results can help foster global knowledge exchange.

\section{Archiving}

This journal uses the LOCKSS system to create a file system that is distributed among collaborating libraries. Those libraries are allowed to create permanent archives of the journal for conservation and restoration.

\section{Ethical responsibilities}

The publication of peer-reviewed articles in a journal is a direct reflection of the work quality of its author(s), as well as of the commitment and qualification of the researchers and investigators who act as evaluators. For this reason, is under the responsibility and duty of the Editorial Board of the journal Alternativas. Cuadernos de Trabajo Social to remember the author(s) that the publication must always be committed to the ethical principles of scientific activity in the following terms:

\section{Publication and authorship}

All the articles must include a list of references, as well as indicate if the authors have received any financial support. The works must be free of plagiarism and scientific misconduct. Both assumptions* are listed below in a non-exhaustive manner:

- Plagiarism: verbatim copy without quotation marks or not citing the source; substantial copy (investigation materials, processes, tables...); to paraphrase or reproduce ideas without citing the source and/or changing its original meaning; to reuse and send your own published texts without indicating the source and constantly paraphrasing. 
- Scientific misconduct: non recognition of all the investigators involved in the elaboration of the work, simultaneously submit to different journals, division of a work in different parts which have the same hypothesis, population and methods, as well as the utilization of false or not proved information. Finally, when the authors send a work, they must announce to the journal the main conflicts of interest.

*Source: http://www.ethics.elsevier.com/

\section{Responsibility of the authors}

The submission of works to Alternativas. Cuadernos de Trabajo Social implies the lecture and acceptance of the editing and publication standards of the journal, included the participation in an anonymous peer-review process.

All the signatories of a work must have contributed in its elaboration in a meaningful way and they agree with its final result and submission. Helping in data collection or taking part in the development of some technique are not enough criteria to figure as an author. In general, to figure as one of the authors, you must fulfil the following requirements:

a) You must have taken part in the conception and realization of the work that has resulted in the article in question.

b) You must have taken part in the writing of the text and in the possible reviews.

c) You must have approved the version which is finally going to be published.

The journal cannot accept responsibility for possible conflicts arising from the authorship of the works to be published.

- The author(s) who have taken part in this work are showed in the article.

- The information used in the article must be real and authentic.

- The authors assume the obligation to correct and/or recant possible mistakes found subsequently.

- The articles must be unpublished and cannot be sent to any other journal simultaneously.

- The author(s) must declare any commercial partnership that could mean a conflict of interest in connection to the article referred. 
- In the section of methodology, the author(s) must mention that, where appropriate, the methods used with the people involved have been carried out with a prior and informed consent.

- Where appropriate, the author(s) must have permission to publish on the part of the funding institution of the research.

- The author(s) are responsible for the obtaining of appropriate licenses in order to reproduce material (text, tables or figures) of other publications partially and quote its source correctly.

- When the experiments carried out on human beings are described, the author(s) must indicate if the procedure followed is in accordance with the ethical standards of the responsible committee on human experimentation (institutional or regional) and with the Declaration of Helsinki of 1975 and reviewed in 2000. Do not include names, initials or any other identification data of the centres where the experiment was carried out.

\section{Review process}

All the articles sent to the journal are subject to a peer-review process with the following characteristics:

- The selection of evaluators is made according to the previous standards and principles based on their qualification, as well as on the quality of their scientific production.

- The review process will be totally anonymous for the authors, as well as for the reviewers. The articles and their reviews will be treated confidentially.

- The reviewers consider, among the assessment criteria, respect for essential ethical principles in scientific research.

- The opinions expressed in the reviews must be objective.

- The authors as well as the reviewers must reveal the relations and sources of funding that could generate potential conflicts of interest.

\section{Editors' responsibility}

- The Editorial Board has the responsibility and authority to accept or refuse an article based on the reviews.

- If necessary, the Editorial Board will reveal the relations or sources of funding that could be considered as potential conflicts of interest regarding the accepted or refused articles. 
- The articles will be only accepted, if there is some evidence of compliance with the editing rules.

- In case of plagiarism, falsification or duplicate publication, as well as the assumptions of scientific misconduct cited above, the Editorial Board reserves the right to withdraw a received, accepted or already published article. In the same way, the publication of corrections and retractions will be promoted against mistakes found.

- The Editorial Board commits itself to preserve the anonymity of the reviewers so that they can never be related to the reviewed articles.

\section{Publication ethical issues}

The Editorial Board commits itself to:

- Protect and preserve the ethical principles of publication.

- Avoid the publication of plagiarized material or elaborated under false pretences.

- Be open to the publication of corrections, clarifications, retractions and apologies, where necessary.

- Provide support in the retraction process of the articles.

- Do whatever is possible to meet the standards of intellectual and ethical commitment.

\section{Exemption from liability}

The Editorial Board as well as the publishing company decline any responsibility for the published material. Neither the Editorial Board nor the publishing company guarantee or support any product that is advertised in the journal or ensure the statements made about that product or service by the manufacturer. 


\section{LISTADO DE EVALUADORES 2016-2017}

$\begin{array}{ll}\text { Aguiar Fernández, Francisco Xabier } & \text { Universidad de Vigo } \\ \text { Aguilar Hendrickson, Manuel } & \text { Universitat de Barcelona } \\ \text { Aguilar Idáñez, Ma José } & \text { Universidad de Castilla-La Mancha } \\ \text { Arias Astray, Andrés } & \text { Universidad Complutense de Madrid } \\ \text { Ballester Laguna, Fernando } & \text { Universidad de Alicante } \\ \text { Bolaños Cartujo, José Ignacio } & \text { Universidad Complutense } \\ \text { Capilla Pérez, Andrea } & \text { Universidad de Huelva } \\ \text { Casas Mínguez, Fernando } & \text { Universidad Castilla La Mancha } \\ \text { Cury, Silvia Patricia } & \text { Universidad Internacional de La Rioja } \\ \text { De la Red Vega, Natividad } & \text { Universidad de Valladolid } \\ \text { Facal Fondo, Teresa } & \text { Universidad de Santiago de Compostela } \\ \text { Fernández Barrera, Josefina } & \text { Universitat de Barcelona } \\ \text { Ferrer Cascales, Rosario Isabel } & \text { Universidad de Alicante } \\ \text { Fombuena Valero, Josefa } & \text { Universitat de València } \\ \text { García Castilla, Francisco Javier } & \text { UnED } \\ \text { Girela Rejón, Blanca } & \text { Universidad de Granada } \\ \text { González Abad, Libertad } & \text { Universidad Complutense Madrid } \\ \text { Jaraiz Arroyo, Germán } & \text { Universidad Pablo de Olavide } \\ \text { La Parra Casado, Daniel } & \text { Universidad de Alicante } \\ \text { López Peláez, Antonio } & \text { UnED } \\ \text { Mesquida González, Josep Maria } & \text { Universidad de Barcelona } \\ \text { Mira-Perceval Pastor, Ma Teresa } & \text { Universidad de Alicante } \\ \text { Morales Villena, Amalia } & \text { Universidad de Granada } \\ \text { Mota López, Rosalía } & \text { Universidad de Comillas } \\ \text { Orgambidez Ramos, Alejandro } & \text { Universidad de Málaga } \\ \text { Pérez Cosín, José Vicente } & \text { Universidad de Valencia } \\ \text { Picornell Lucas, Antonia } & \text { Universidad de Salamanca } \\ \text { Rivera Álvarez, Joaquín María } & \text { Universidad Complutense } \\ \text { Ródenas Rigla, Francisco } & \text { Universidad de Valencia } \\ \text { Rodríguez Berrio, Arantzazu } & \text { Universidad de Deusto } \\ & \end{array}$


Sancho Salido, Jordi

Santana Hernández, Juana Dolores

Segado Sánchez-Cabezudo, Sagrario

Sotomayor Morales, Eva

Taboada González, María Luisa

Verde Diego, Carmen

Villalba Quesada, Cristina

Yeves Bou, $\mathrm{M}^{\mathrm{a}}$ Teresa
Universitat de Barcelona

Universidad de la Laguna

UNED

Universidad de Jaén

TS y prof emerita U. Málaga

Universidad de Vigo

Universidad Pablo de Olavide

Universidad de Valencia 



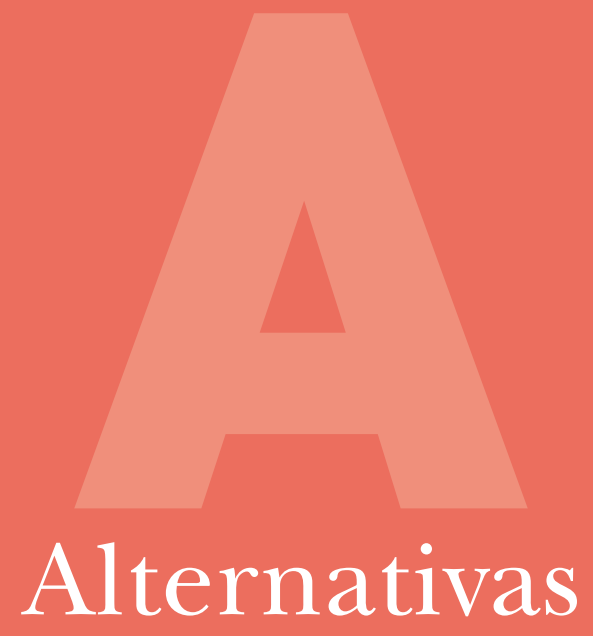

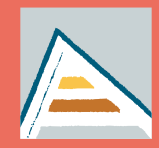 \\ Universitat d'Alacant \\ Universidad de Alicante
}

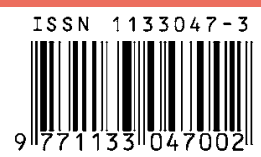

